\title{
Supramolecular \\ Tunneling Junctions
}
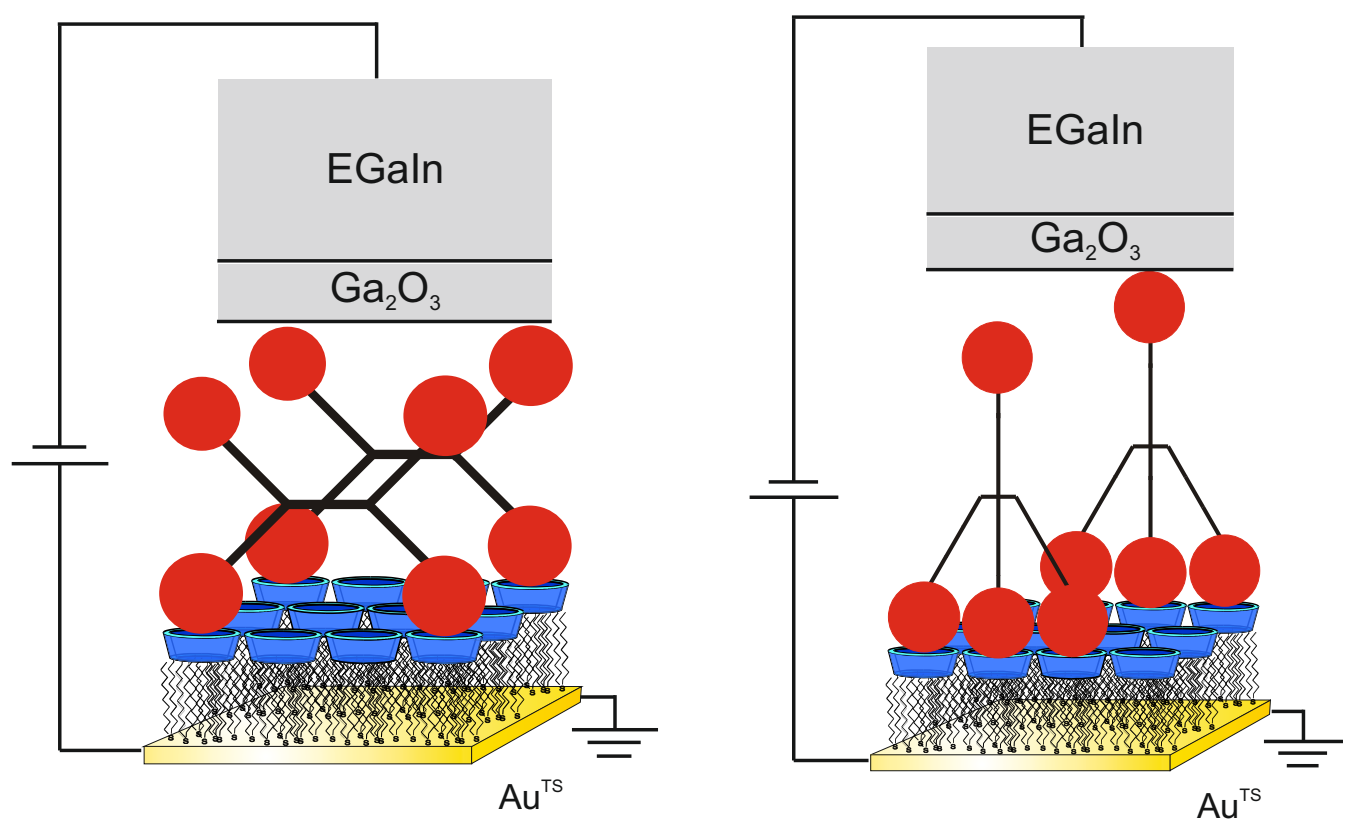

Kim Wimbush 


\section{SUPRAMOLECULAR TUNNELING JUNCTIONS}

Kim Wimbush 
Thesis committee members:

\begin{tabular}{|c|c|}
\hline Prof.dr. P.J. Kelly & University of Twente (Chairman) \\
\hline Prof.dr.ir. D.N. Reinhoudt & University of Twente (Promotor) \\
\hline Prof.dr. A.H. Velders & Wageningen University (Promotor) \\
\hline Dr. C.A. Nijhuis & $\begin{array}{l}\text { National University of Singapore (Assistant- } \\
\text { Promotor) }\end{array}$ \\
\hline Prof.dr.ir. W.G. van der Wiel & University of Twente \\
\hline Prof.dr.ir. H.J.W. Zandvliet & University of Twente \\
\hline Prof.dr.ir J. Huskens & University of Twente \\
\hline Prof.dr. D.M. de Leeuw & University of Groningen / Philips \\
\hline
\end{tabular}

The research described in this thesis was financially supported by the MESA+ Institute for Nanotechnology and NanoNed, a national nanotechnology program coordinated by the Dutch Ministry of Economic Affairs.

Publisher: Ipskamp, Drukkers, Enschede, The Netherlands ISBN: 978-90-365-3468-0

DOI: $10.3990 . / 1.9789036534680$

URL: http://dx.doi.org/10.3990/1.9789036534680

Copyright C Kim Stuart Wimbush, Enschede, 2012.

All rights reserved. No part of this work may be reproduced by print, photocopy or any other means without prior permission in writing from the author 


\title{
SUPRAMOLECULAR TUNNELING JUNCTIONS
}

\author{
DISSERTATION \\ to obtain \\ the degree of doctor at the University of Twente, \\ on the authority of the rector magnificus, \\ Prof.dr. H. Brinksma, \\ on account of the decision of the graduation committee, \\ to be publicly defended \\ on Thursday the $29^{\text {th }}$ of November, 2012 at $12.45 \mathrm{~h}$
}

by

\section{Kim Stuart Wimbush}

Born on the $3^{\text {rd }}$ of June, 1984

in Campbelltown, South Australia, Australia 
This dissertation has been approved by:

Promotors: $\quad$ Prof.dr.ir. D.N. Reinhoudt

Prof.dr. A.H. Velders

Assistant Promotor: Dr. C.A. Nijhuis 
To all my family and friends! 


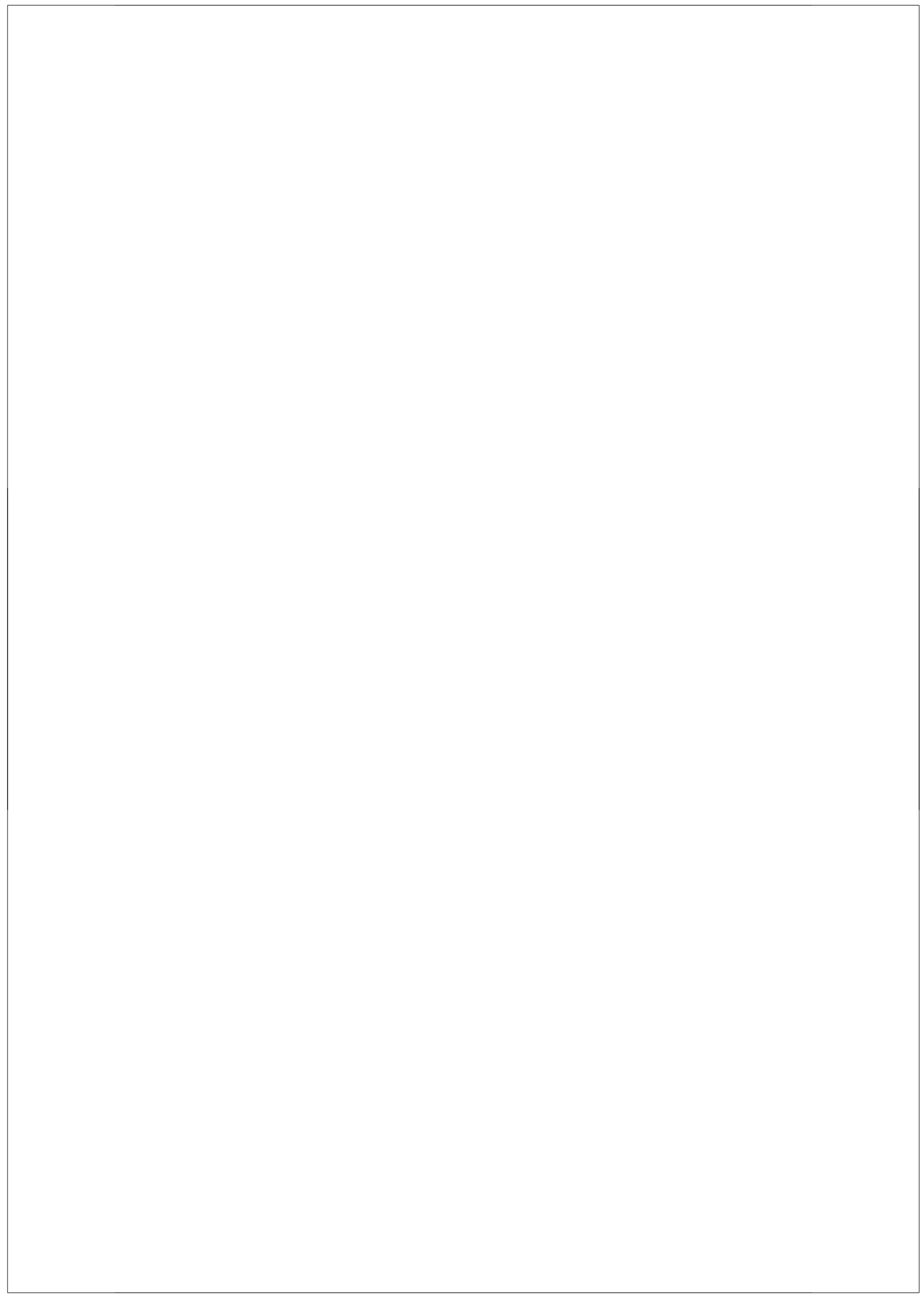




\section{Table of Contents}

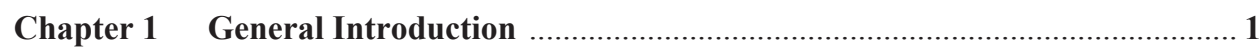

Chapter 2 Self-Assembled Monolayers in Large Area Molecular Tunneling

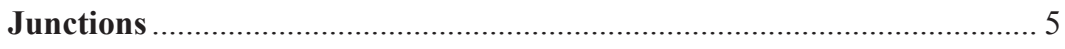

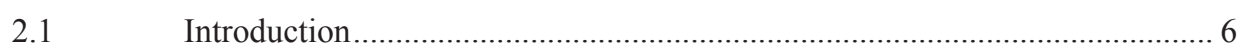

2.2 The Components of a Molecular Tunneling Junction...................................... 8

2.2.1 Bottom Electrode ..................................................................................... 9

2.2.2 The Molecular Layer ........................................................................................ 12

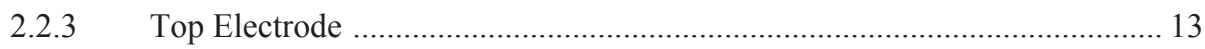

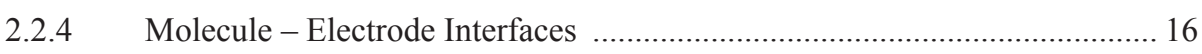

2.3 The Role of SAMs in Charge Transport in Various Large Area Molecular

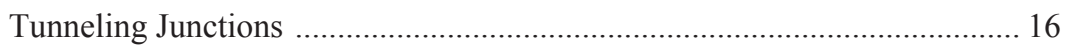

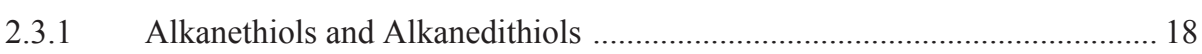

2.3.1.1 EGaIn Tunneling Junctions ......................................................... 20

2.3.1.2 PEDOT:PSS Tunneling Junctions ……............................................ 21

2.3.1.3 Graphene Tunneling Junctions ………............................................. 23

2.3.1.4 Mercury Drop Tunneling Junctions .................................................. 24

2.3.1.5 Hybrid Tunneling Junctions .............................................................. 27

2.3.1.6 Metal Evaporated Tunneling Junctions _............................................ 29

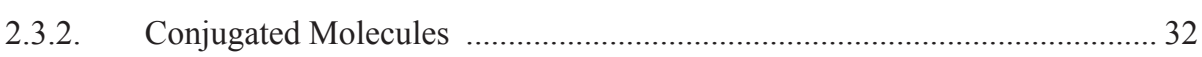

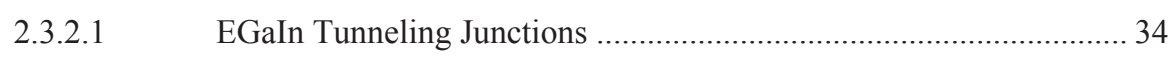

2.3.2.2 PEDOT:PSS Tunneling Junctions ....................................................... 35

2.3.2.3 Mercury Drop Tunneling Junctions ............................................... 39

2.3.2.4 Hybrid Tunneling Junctions ……………………………………..... 42

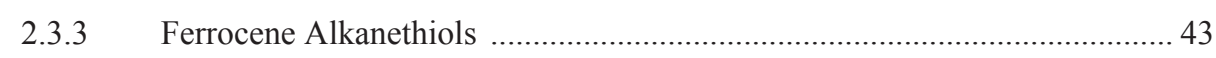

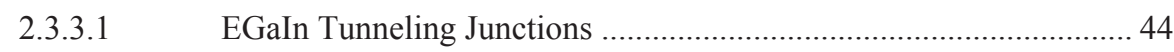

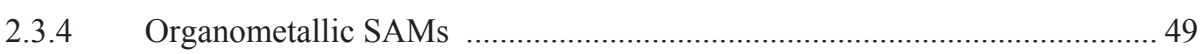

2.3.4.1 EGaIn Tunneling Junctions ........................................................ 51

2.3.4.2 PEDOT:PSS Tunneling Junction ………………………………........ 53

2.3.4.3 Graphene Tunneling Junctions …………………………………......... 56 


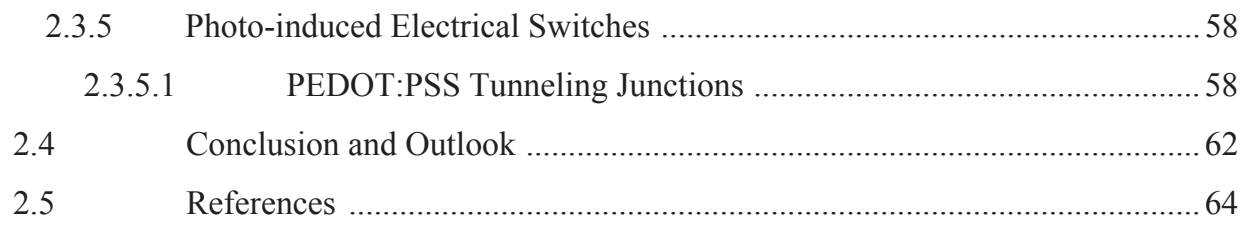

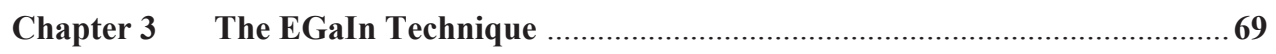

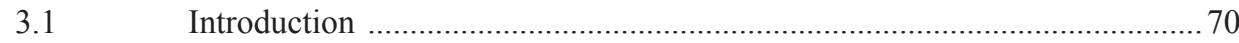

Construction of the EGaIn Technique ....................................................... 82

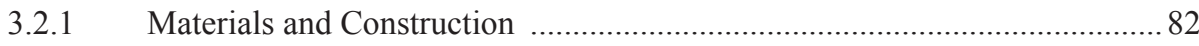

3.2.2 Wiring and Connecting to the Electrometer/Remote Source Meter (Keithley) and Computer .................................................................................. 85

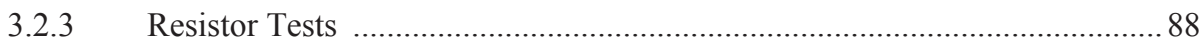

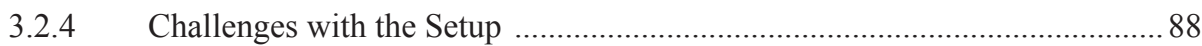

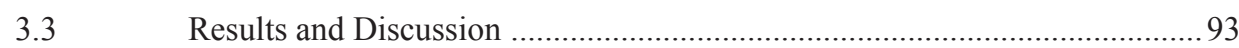

3.3.1 Performing Measurements on Molecular Tunneling Junctions ....................93

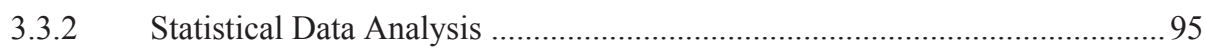

3.3.3 Importance of Statistically Relevant Numbers of Data ............................... 97

3.3.4 Minimizing Defects in Supramolecular Tunneling Junctions ........................ 98

3.3.5 The Use of Rectification to Investigate $J(V)$ Characteristics ...................... 100

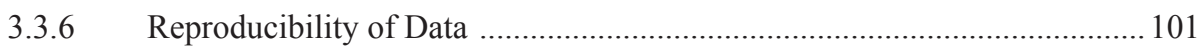

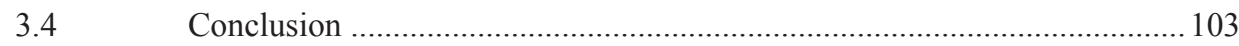

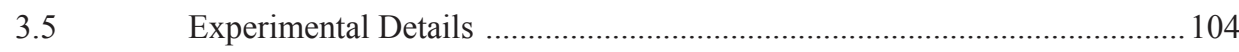

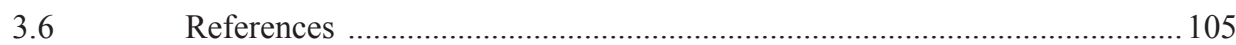

Chapter 4 Control Over Rectification in Supramolecular Tunneling Junctions: Poly(propylene) imine Dendrimers ...................................................109

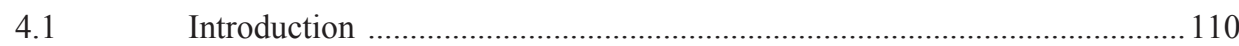

4.2 Construction of the Supramolecular Tunneling Junctions : Generation one Poly(propylene) imine Dendrimers ...................................................... 112

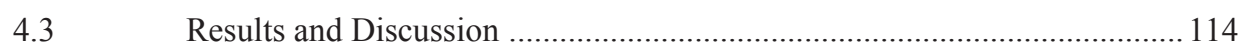

4.3.1 $J(V)$ Data Accumulation and Statistical Analysis ..................................... 114

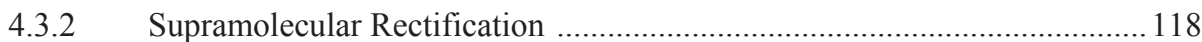

4.3.3 Mechanism of Charge Transport ….......................................................... 120

4.3.4 Current Density and Hysteresis .......................................................... 123

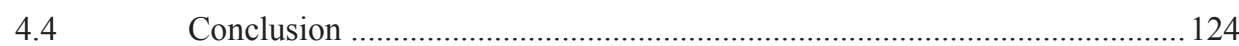




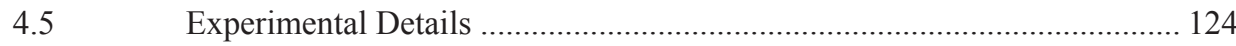

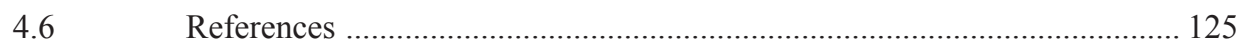

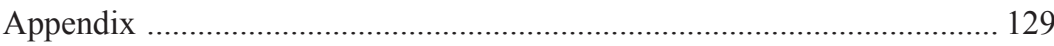

Chapter 5 Control Over Rectification in Supramolecular Tunneling Junctions: Poly(amido amine) Dendrimers ......................................................... 131

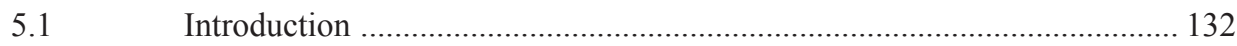

5.2 Construction of the Supramolecular Tunneling Junctions : Generation zero Poly(amido amine) Dendrimers ............................................................ 134

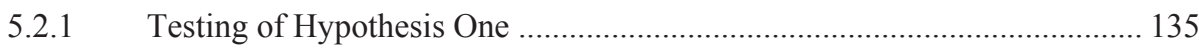

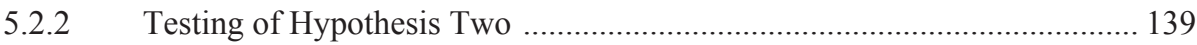

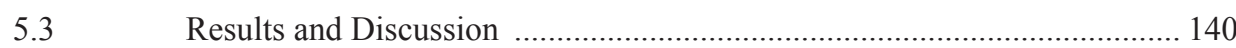

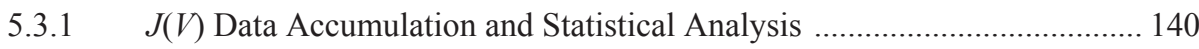

5.3.2 Verification of Hypothesis One ............................................................... 145

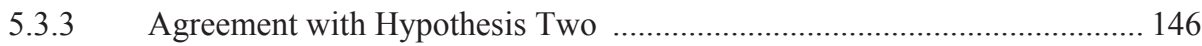

5.3.4 Comparison of Control Dendrimers ....................................................... 147

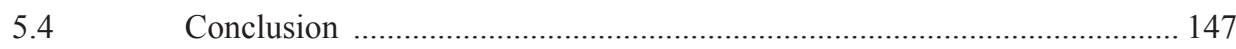

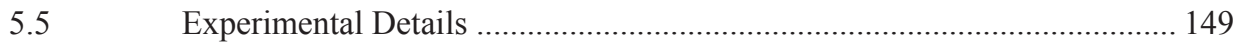

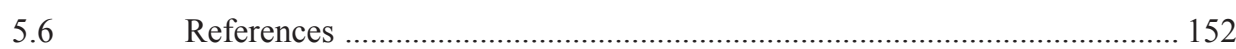

Chapter 6 Voltage Induced Rectification in EGaIn Supramolecular Tunneling

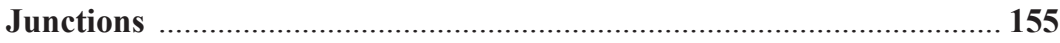

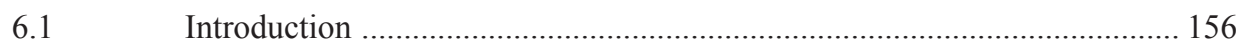

6.2 Construction of the Supramolecular Tunneling Junctions: Generation One Poly(propylene) imine Dendrimers and Generation Zero Poly(amido amine)

Dendrimers 157

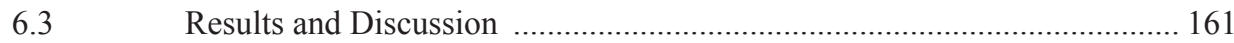

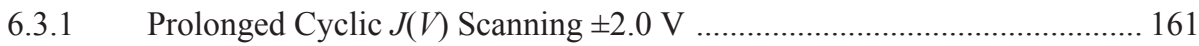

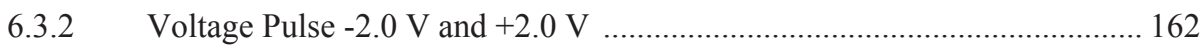

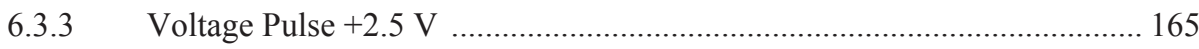

6.3.4 Rectification Measured vs. Various Voltage Pulses .................................. 166

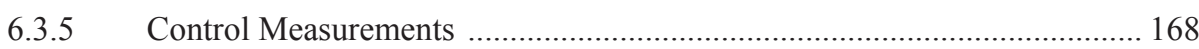

6.3.6 The Origin of the Increase in $R$ in EGaIn Supramolecular Tunneling

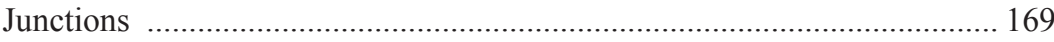

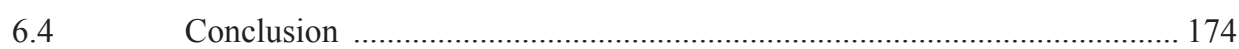

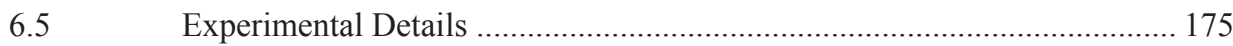


Table of Contents

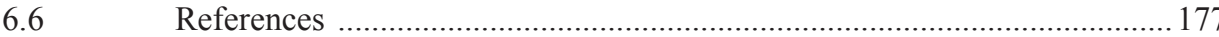

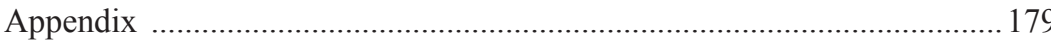

Chapter 7 Electron Induced Dynamics of Heptathioether $\beta$-cyclodextrin

Molecules ................................................................................. 183

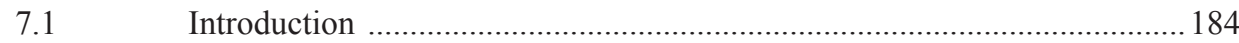

7.2 The Scanning Tunneling Microscope (STM) Setup ................................. 186

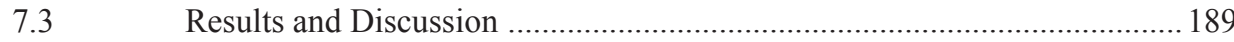

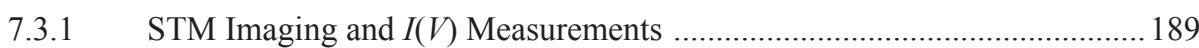

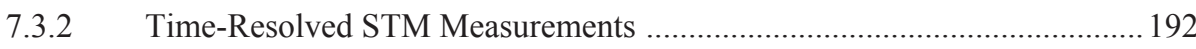

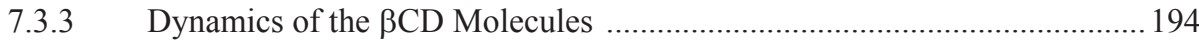

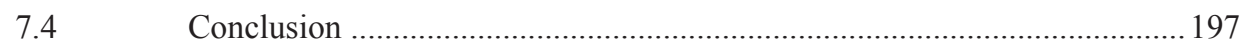

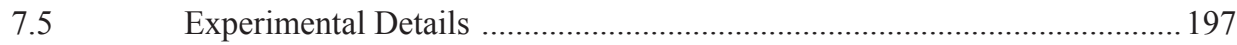

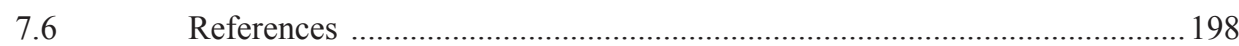

Appendix

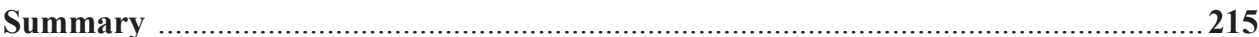

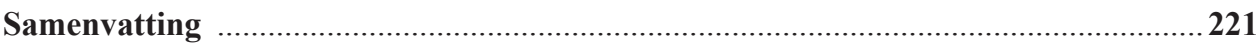

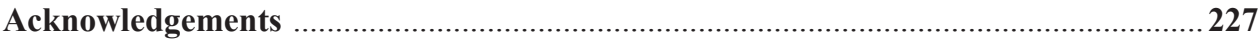

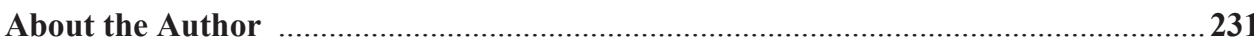




\section{Chapter 1}

\section{General Introduction}

Molecular Electronics is a multidisciplinary field created by the pioneering experimental work of Mann and Kuhn ${ }^{[1]}$ and Polymeropoulos, ${ }^{[2]}$ and the visionary theory of Aviram and Ratner. ${ }^{[3]}$ They proposed the use of a single molecule as the functional component in an electronic device. This would be the ultimate solution to the fundamental top-down fabrication limitations of the semiconductor industry. In 2000, it was reported that if the miniaturization of microprocessor components such as transistors continued to follow Moore's Law (which states that the number of transistors that can be packed onto a microprocessor doubles every 18-20 months ${ }^{[4]}$ ) conventional silicon chips would reach their physical limitation around the year 2012. ${ }^{[5]}$ These foreseen problems with the semiconductor industry generated accelerated interest and research in the field of Molecular Electronics, leading it to become Sciences 'breakthrough of the year' in 2001. ${ }^{[6]}$ However, by 2003 the field of Molecular Electronics was suffering a midlife crisis, ${ }^{[7]}$ as the originally reported molecular driven functions were found to be no more than extrinsic effects, such as the formation and dissolution of metal filaments along the single layer of molecules, i.e. 'monolayer'. During this period of time the semiconductor industry was also not standing still, and by the end of 2011 had already fabricated transistors with a minimum feature size of $28 \mathrm{~nm}$, and proposed methods to fabricate a new generation of transistors leading to smaller minimum feature sizes that could rival single molecules. ${ }^{[8]}$ The difficulties faced with molecular electronic technologies along with this continual development of the semiconductor industry, have led the field of molecular electronics to re-evaluate their experimental paradigms and re-focus its intended role in functioning devices.

As the miniaturization of the semiconductor microprocessor components is still becoming ever increasingly more expensive, there remains a role for molecular electronic technology. Instead of creating smaller or single molecule devices, more cost-effective devices can be created using the bottom-up approach of self-assembly, or more specifically, molecular selfassembly. Molecular self-assembly can be defined as the process in which molecules (or parts of molecules) spontaneously arrange themselves into ordered ensembles without human intervention. ${ }^{[9]}$ This process is crucial in the formation of a single layer of molecules, 
i.e. 'monolayer', on a solid or liquid surface, with these entities being known as selfassembled monolayers (SAMs). ${ }^{[10]}$

The self-assembly of heptathioether functionalized $\beta$-cyclodextrin $(\beta C D)$ on $A u$, creates a well-defined, hexagonally packed, $\beta \mathrm{CD}$ monolayer, ${ }^{[1]]}$ which is coined as a 'supramolecular platform'. In this thesis, the supramolecular platform is used as the basis along with a top electrode of Eutectic Gallium Indium (EGaIn), to create supramolecular tunneling junctions. These supramolecular junctions allow for the investigation of the charge transport of the adsorbed dendritic molecular architectures that vary in core structure, and/or terminal functionality, as well as all of the individual junction components.

In Chapter 2, an overview is given of the charge transport characteristics of different selfassembled monolayers (SAMs) in a variety of two-terminal large-area $\left(>1 \mu \mathrm{m}^{2}\right)$ molecular tunneling junction assemblies. Here the components that comprise a two-terminal molecular junction are also introduced, along with explanations of how they can affect the SAMs charge transport characteristics.

Chapter 3 describes the construction of the Eutectic Gallium Indium (EGaIn) setup at the University of Twente, based on the EGaIn technique developed in the laboratories of Whitesides and co-workers. ${ }^{[12]}$ It includes a list of all parts ordered, calibration measurements performed and difficulties encountered. This chapter also includes a discussion about the different techniques used to create two-terminal large-area $\left(>1 \mu \mathrm{m}^{2}\right)$ SAM based tunneling junctions and compares their advantages and disadvantages.

In Chapter 4, the rectification ratio $(R)$, where $R=|J(-2.0 \mathrm{~V})||| J(+2.0 \mathrm{~V}) \mid$, is used to compare the charge transport characteristics of different terminal functionalized poly(propylene) imine (PPI) dendrimers adsorbed on the supramolecular platform ${ }^{[13]}$ in EGaIn tunneling junctions. Statistically relevant numbers of data show that the value of $R$ obtained is dependent on the terminal functional moiety of the dendrimer. The results presented along with the hypothesis given for the mechanism of charge transport, indicate that the rectification observed is molecular in origin.

Chapter 5 uses $R$ to compare the charge transport characteristics of different functionalized poly(amido amine) (PAMAM) dendrimers absorbed on the supramolecular platform in EGaIn tunneling junctions. The use of the PAMAM dendrimers allows for the investigation 2 
of hypotheses formulated in Chapter 4, by varying the position of the terminal functional moiety of the dendrimer within the junction and by changing the packing density of the dendrimer layer formed on the supramolecular platform.

In Chapter 6, the experimental limitations of the EGaIn technique as a 'non-active' component in supramolecular tunneling junctions are investigated. The excellent stability of the supramolecular tunneling junctions allows for voltage pulses to be applied at a variety of biases for different periods of time. The resultant values of $R$ are found to be dependent upon the voltage and length of time of the voltage pulse.

In chapter 7, Scanning Tunneling Microscopy (STM) and Spectroscopy (STS) are used to investigate the charge transport characteristics and hence the dynamical behavior of the heptathioether functionalized $\beta C D$ molecules within the supramolecular platform. The supramolecular platform is found to exhibit rich dynamical behavior that increases with increasing tunneling current and sample bias.

The appendix presents the values of $R$ found for higher generations of PPI dendrimers adsorbed on the supramolecular platform in EGaIn tunneling junctions. Also shown, is the ability of EGaIn supramolecular tunneling junctions to behave as 'memristors'. Finally presented, are the results obtained during a collaboration with de Leeuw and co-workers at the University of Groningen, where the $J(V)$ characteristics of the supramolecular $\beta C D$ dendrimer assemblies were investigated in poly(3,4-ethylenedioxythiophene):poly(4styrenesulphonic acid) (PEDOT:PSS) tunneling junctions.

\section{References}

[1] B. Mann, H. Kuhn, J. Appl. Phys. 1971, 42, 4398-4405.

[2] E. E. Polymeropoulos, J. Appl. Phys. 1977, 48, 2404-2407.

[3] A. Aviram, M. A. Ratner, Chem. Phys. Lett. 1974, 29, 277-283.

[4] G. E. Moore, Proc. IEEE 1998, 86, 82-85.

[5] P. Ball, Nature 2000, 406, 118-120.

[6] R. F. Service, Science 2001, 294, 2442-2443.

[7] R. F. Service, Science 2003, 302, 556-559.

[8] I. Ferain, C. A. Colinge, J.-P. Colinge, Nature 2011, 479, 310-316. 
[9] a)G. M. Whitesides, M. Boncheva, Proc. Natl. Acad. Sci. U. S. A. 2002, 99, 4769-4774; b)G. M. Whitesides, Sci.Am. 1995, 273, 146-149; c)G. M. Whitesides, B. Grzybowski, Science 2002, 295, 2418-2421.

[10] J. C. Love, L. A. Estroff, J. K. Kriebel, R. G. Nuzzo, G. M. Whitesides, Chem. Rev. 2005, 105, 1103-1169.

[11] a)M. W. J. Beulen, J. Bugler, B. Lammerink, F. A. J. Geurts, E. M. E. F. Biemond, K. G. C. van Leerdam, F. C. J. M. van Veggel, J. F. J. Engbersen, D. N. Reinhoudt, Langmuir 1998, 14 6424-6429; b)M. W. J. Beulen, J. Bügler, M. R. de Jong, B. Lammerink, J. Huskens, H. Schönherr, G. J. Vancso, B. A. Boukamp, H. Wieder, A. Offenhäuser, W. Knol, F. C. J. M. van Veggel, D. N. Reinhoudt, Chem. Eur. J. 2000, 6, 1176-1183.

[12] R. C. Chiechi, E. A. Weiss, M. D. Dickey, G. M. Whitesides, Angew. Chem. Int. Ed. 2008, 47, 142-144.

[13] a)C. A. Nijhuis, J. Huskens, D. N. Reinhoudt, J. Am. Chem. Soc. 2004, 126, 12266-12267; b)C. A. Nijhuis, F. Yu, W. Knoll, J. Huskens, D. N. Reinhoudt, Langmuir 2005, 21, 7866-7876; c)C. A. Nijhuis, B. A. Boukamp, B. J. Ravoo, J. Huskens, D. N. Reinhoudt, J. Phys. Chem. C 2007, 111, 9799-9810; d)C. A. Nijhuis, K. A. Dolatowska, B. J. Ravoo, J. Huskens, D. N. Reinhoudt, Chem. Eur. J. 2007, 13, 69-80. 
Chapter 2

\section{Self-Assembled Monolayers in Large Area Molecular Tunneling Junctions}

This chapter reviews the charge transport characteristics of different self-assembled monolayers (SAMs) in a variety of two-terminal large-area $\left(>1 \mu \mathrm{m}^{2}\right)$ molecular tunneling junctions. Included within the review is a brief description of each component that comprises the molecular tunneling junctions and how it influences the charge transport characteristics of the SAM. 


\subsection{Introduction}

Molecular self-assembly can be defined as the process in which molecules (or parts of molecules) spontaneously arrange themselves into ordered ensembles without human intervention. ${ }^{[1]}$ The chemical structure of the molecules themselves determines the structure of the assembly, with non-covalent interactions typically being the driving force. One of the most investigated self-assembled systems is the self-assembled monolayer (SAM). A SAM is a single layer formed by the adsorption of molecules from solution or the gas phase onto the surface of solids, or on the surface of liquids (in the case of mercury and other liquid metals and alloys). ${ }^{[2]}$ The molecules organize spontaneously (and sometimes epitaxially) into crystalline (or semi-crystalline) structures. ${ }^{[2]}$ Typical molecules used to form SAMs consist of three parts: (1) a chemically active headgroup which has a specific affinity for a substrate; (2) an organic phase/spacer which determines the thickness of the layer, serves as a physical barrier and can change the electronic and optical properties; (3) a terminal functional group that couples the molecule and thus the SAM to the external environment and determines the SAMs properties (e.g. hydrophobic, hydrophilic, electrically/optically active, etc.). ${ }^{[2]}$ As SAMs can form well-defined and ordered layers, and their properties can be easily tailored by the design and synthesis of the molecules used to form them, SAMs have become of great interest to the field of molecular electronics. Studies of SAMs in molecular tunneling junctions have aimed to gain a greater understanding of the mechanism of charge transport across, and thus the function of the monolayer and in turn create cheap functional devices that in the future could possibly replace or be incorporated into semiconductor technology. ${ }^{[3]}$

This Chapter reviews the function of self-assembled monolayers (SAMs) in various large-area molecular tunneling junctions, with large-area being $>1 \mu \mathrm{m}^{2}$, and the scope of the review is limited to SAMs formed on metal bottom electrodes. Therefore, even though important to the continual development of the field of molecular electronics, small-area junctions ${ }^{[4]}$ formed using scanning tunneling microscopy $(\mathrm{STM}),{ }^{[5]}$ conductive probe atomic force microscopy $(\mathrm{CP}-\mathrm{AFM}),{ }^{[6]}$ break junctions, ${ }^{[7]}$ crossed wire junctions $^{[8]}$ and nanopores ${ }^{[9]}$ will not be discussed in detail, nor will SAMs formed on semiconducting bottom electrode surfaces $^{[10]}$ and mono or multi molecular layers covalently bound to a graphite carbon substrate, ${ }^{[11]}$ or molecular layers formed using the langmuir blodgett technique. ${ }^{[12]}$ 
A variety of terminology will be used throughout this review. The definitions to these terms are as follows:

- Molecular tunneling junction: A device consisting of a top electrode and a bottom electrode that are separated by a molecular layer.

- Root mean square (RMS): A mathematical term which is the square root of the arithmetic mean (average) of the squares of the original values.

- Packing density: The number of molecules present in a given area on the surface.

- Current (I) vs. Voltage (V) scan/plot, (I(V)) scan/plot: Electrical current measured in ampere as a function of the applied voltage.

- Current density $(J)$ vs. Voltage $(V)$ scan/plot, $(J(V))$ scan/plot: Electrical current measured in ampere per unit area as a function of the applied voltage.

- Normalized resistance $(R S)$ vs. Voltage (V) scan/plot, $(R S(V))$ scan/plot: Electrical resistance measured in ohm per unit area as a function of the applied voltage.

- Tunneling decay coefficient ( $\beta$ ): Quantifies the decay of the electron tunneling probability with increasing distance (per Angstrom $\left(\AA^{-1}\right)$ ) between the electrodes. ${ }^{[13]}$ In some studies the distance between the electrodes is given as per carbon atom $\left(n_{\mathrm{c}}{ }^{-1}\right)$ of the molecule investigated. In this chapter, to keep the units of $\beta$ reported consistent, when discussing studies that report $\beta$ as $n_{\mathrm{c}}{ }^{-1}, \beta$ has also been given as $\AA^{-1}$.

- Working junction: A molecular tunneling junction that gives reproducible $J(V)$ data within the specified experimental error of $J$, without forming a short circuit and thus showing reliable $J(V)$ characteristics of the molecular layer. Please note that there is no universal definition of a working junction, and that this definition may differ in different studies.

- Short circuit: Junction in which metal filaments have penetrated through the molecular layer, causing the top and bottom electrode to be in direct electrical 
contact with each other which upon applying a voltage produces an ohmic $J(V)$ curve.

- Breakdown voltage (BDV): The applied voltage at which a working junction becomes a short circuit.

- Breakdown field (BDF): The applied electric field at which a working junction becomes a short circuit.

- Yield of working junctions: The number of working junctions divided by the number of junctions investigated $\times 100$.

- Chemisorbed: Where the SAM is chemically attached to the bottom electrode.

- Physisorbed: Where the SAM is in contact with the electrode through intermolecular forces such as van der Waals forces.

Below, an overview of the components that comprise a molecular tunneling junction is given. As there is not one standard technique used to create the top electrodes in large-area molecular tunneling junctions, each type of top electrode will be described along with a brief discussion with the problems still associated with each of these techniques. This will be followed by a detailed discussion of SAMs that function as either a dielectric, semiconductor, diode or a switch/memory device and their electrical characteristics in a variety of large-area molecular tunneling junctions.

\subsection{The Components of a Molecular Tunneling Junction}

Figure 2.1 shows a simplified schematic of the components that comprise a molecular tunneling junction: the bottom electrode, the top electrode, and the molecular layer immobilized between the two (metal)electrodes, along with the two interfaces of these components, i.e. 'electrode-molecule' interfaces. Each individual element and both of the interfaces are a key aspect in the performance of the junction. Within the scope of the review, examples of each component and the different types of interfaces will be given and briefly discussed along with the role that they play within the molecular tunneling junction. 


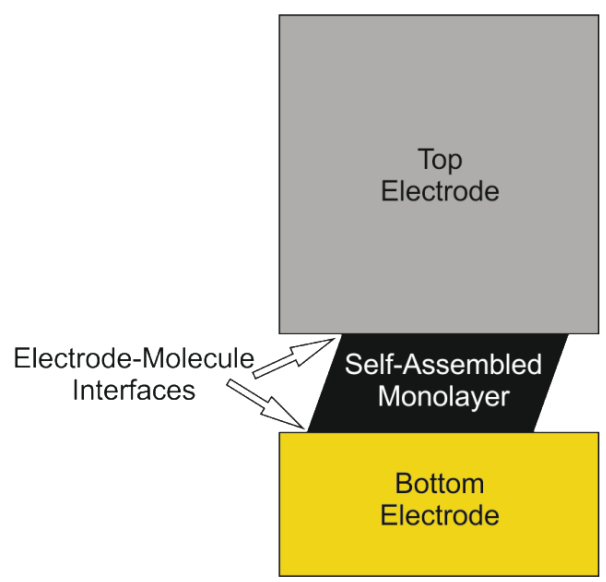

Figure 2.1: A schematic of a molecular tunneling junction, which is comprised of a bottom electrode, top electrode and self-assembled monolayer (molecular layer). The two electrode-molecule interfaces are depicted as they are also of importance when defining the molecular tunneling junction's properties.

\subsubsection{Bottom Electrode}

The bottom electrode is a metallic surface, such as $\mathrm{Au}, \mathrm{Ag}, \mathrm{Pt}, \mathrm{Pd}, \mathrm{Hg}$, etc., that the SAM will be adsorbed to. To optimize the formation of working devices, great care must be taken when fabricating the bottom electrode. The atomic structure of the metal used and the roughness of the surface influences the molecular orientation, (i.e. tilt angle), packing density of the SAMs, and the distance between the bottom and top electrodes, which in turn strongly dominates the Current Density $(J)$ vs. Voltage $(V)(J(V))$ characteristics measured. ${ }^{[2,14]}$ Additionally, the bottom electrode must be as clean as possible, because even though molecules that form self-assembled layers can eventually displace most adsorbed impurities, these impurities may inhibit the formation or increase the formation time of densely packed, well-defined SAMs. ${ }^{[2]}$

Typical methods used in the past to fabricate the bottom electrode generally only required vapor deposition of the metal onto either Si, glass, or mica, that contained an adhesion layer. However, over an area of $25 \mu^{2}$ the root mean square (RMS) roughness of the vapor-deposited surfaces is $5.1 \mathrm{~nm}$ and $4.1 \mathrm{~nm}$ for $\mathrm{Au}$ and $\mathrm{Ag}$, respectively (Figure 2.2a). ${ }^{[15]}$ Also, the vapor-deposited surfaces require additional cleaning steps before use, such as immersion in a piranha solution. As SAMs are typically $2 \mathrm{~nm}$ thick, the roughness of a vapor-deposited bottom electrode, will influence the packing density of the SAM and the 
distance between the top and bottom electrode, and thus, will dominate the $J(V)$ characteristics, causing short circuits and irreproducible results. The vapor-deposited $\mathrm{Au}$ metal films can be 'flattened' by flame annealing. ${ }^{[16]}$ This produces areas of atomically flat $\mathrm{Au}(111)$, which are separated by large step edges and grain boundaries (Figure 2.2b). Although metal surfaces prepared in this manner are perfect for single-molecule measurements, for large-area molecular tunneling junctions, they are not optimal. The large step edges and grain boundaries produce a 'rough surface', with a RMS of $1.4 \mathrm{~nm}$ over an area of $25 \mu^{2}{ }^{[15]}$ Flame annealing does not work for metal films such as Ag, as after annealing the RMS of the surface is $6.2 \mathrm{~nm}$ over an area of $25 \mu \mathrm{m}^{2} .^{[15]}$ However, ultra-flat surfaces can be created for a wide range of metals by using a procedure known as template stripping. ${ }^{[15,17]}$ This procedure allows metal surfaces to have the same ultra-flat topography as substrates such as $\mathrm{Si}$ and mica, with template stripped $\mathrm{Au}$ and $\mathrm{Ag}$ surfaces being produced with RMS values of $0.6 \mathrm{~nm}$ and $1.2 \mathrm{~nm}$, respectively, over an area of $25 \mu \mathrm{m}^{2}$. An example of how template stripping is carried out is described below and shown in Figure 2.2c, with an AFM image displaying the ultra-flat nature of the surface shown in Figure 2.2d. For template stripping, the metal of choice is deposited onto a $\mathrm{Si} / \mathrm{SiO}_{2}$ substrate by evaporation with an e-beam. A glass slide is then attached to the surface of the metal using an adhesive. The adhesive is then cured by exposing it to UV light for 1 hour, or by heating at $150^{\circ} \mathrm{C}$ for 1 hour, firmly binding the glass slide to the surface of the metal. A razor is used to cleave the glass/adhesive/metal composite from the $\mathrm{Si} / \mathrm{SiO}_{2}$ template, exposing the ultra-flat surface of the metal that was at the metal $/ \mathrm{SiO}_{2}$ interface. ${ }^{[15]}$ Additionally, the exposed metal surface is very clean, as the metal is evaporated onto the $\mathrm{Si} / \mathrm{SiO}_{2}$ substrate under vacuum, and therefore the only time the metal at the metal $/ \mathrm{SiO}_{2}$ interface is exposed to ambient air, is upon cleaving from the $\mathrm{Si} / \mathrm{SiO}_{2}$ substrate. Other variations of this procedure have been published. ${ }^{[17]}$ 
a)

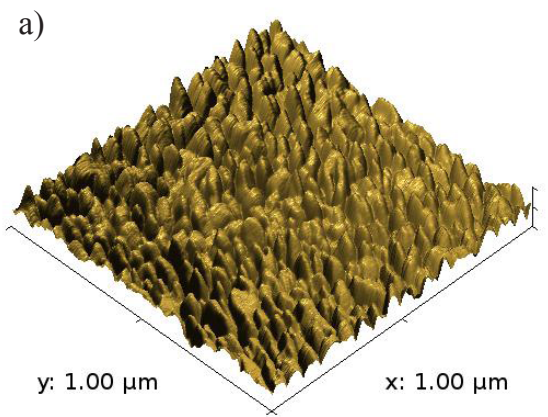

c)

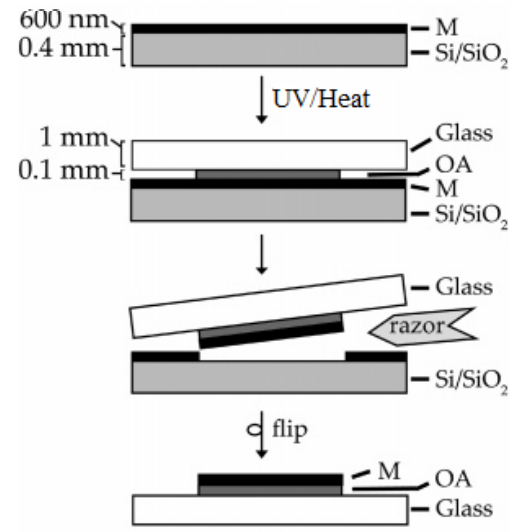

b) $19 \mathrm{~nm}$ $0 \mathrm{~nm}$

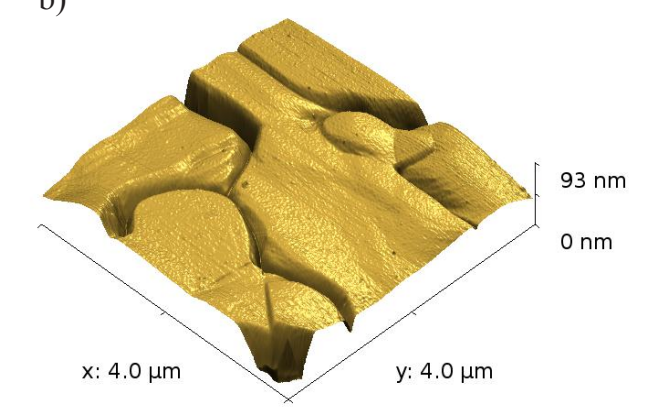

d)

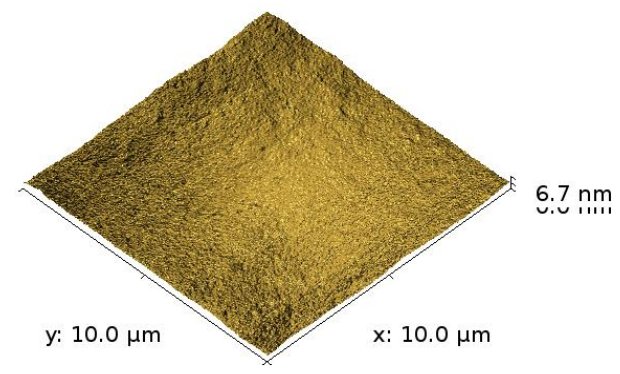

Figure 2.2: AFM images of Au surface prepared via (a) metal vapor deposition (AFM image $1.0 \mu \mathrm{m}$ by 1.0 $\mu \mathrm{m}$ ), (b) flame annealing (AFM image $4.0 \mu \mathrm{m}$ by $4.0 \mu \mathrm{m}$ ), (d) template stripping (AFM image $10 \mu \mathrm{m}$ by 10 $\mu \mathrm{m}$ ). (c) Schematic of the template stripping procedure where $\mathrm{OA}=$ optical adhesive and $\mathrm{M}=$ metal. (AFM images in Figures (a), (b) and (d) were performed by Alberto Gomez Casado from the Molecular Nanofabrication group at the University of Twente in the Netherlands. Figure (c) reprinted with permission from. ${ }^{[15]}$ Copyright $(2007$ American Chemical Society).

Although template stripped bottom electrode surfaces are 'ultra-flat', they are still not flat enough to be completely non-influential in the $J(V)$ characteristics measured in large-area tunneling junctions. Defects present such as step edges, grains, pin holes, impurities, and even residual surface roughness affect the $J(V)$ characteristics. ${ }^{[13 b]}$ However, to date, template stripped surfaces are the flattest and hence the best surfaces to work with when forming SAMs on metal surface electrodes, in large-area tunneling junctions (Figure 2.3). 


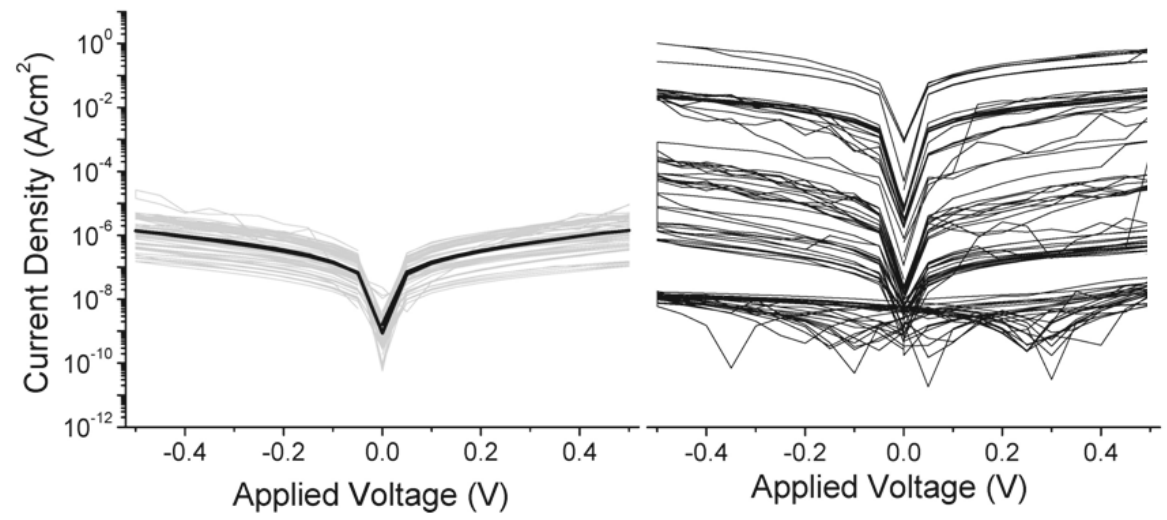

Figure 2.3: (left) Semi-log plot of the averaged $|J|(V)$ data (log-mean, bold black line) and raw $|J|(V)$ data (light grey lines) measured $\pm 0.5 \mathrm{~V}$, for a single layer of molecules (monolayer) immobilized on template stripped Ag surfaces, within tunneling junctions. (right) Same measurements performed, however, the monolayer was immobilized on vapor-deposited (V-DEP) Ag surfaces (Reprinted with permission from. ${ }^{[13 b]}$ Copyright (C) 2007 American Chemical Society).

\subsubsection{The Molecular Layer}

When the 'traditionally hypothesized' molecular tunneling junction is formed correctly, the molecular layer immobilized in between the electrodes should function as the active component of the junction. Depending on the type of molecule used, the molecular layer can function as either a dielectric, a semiconductor, a diode or a switch/memory device. Within the scope of this review, only molecular tunneling junctions comprised of well-defined single molecular layers that self-assemble onto the bottom electrode, i.e., Self-Assembled Monolayers (SAMs), are discussed. As discussed in the introduction (section 2.1) the molecules used to form SAMs consist of a chemically active headgroup, an organic phase/spacer and a terminal functional group. ${ }^{[2]}$ For SAMs used in molecular tunneling junctions, the headgroup is generally a thiol moiety due to its strong affinity for the bottom metal electrode. The organic phase/spacer is typically a repetitive organic unit such as $\left(\mathrm{CH}_{2}\right)_{\mathrm{n}}$, where $\mathrm{n}$ equals the number of units which controls the thickness of the layer and hence, the distance between the two electrodes. Occasionally, within the organic phase/spacer a chemical functional group may be incorporated as the intended active 12 
component of the molecule. Finally, the terminal functional group can act as either the active component of the molecule, or provide the appropriate functionality to optimize the contact with the top electrode. In section 2.3 of this review, examples of SAMs that function as a dielectric (alkanethiols and alkanedithiols), a semiconductor ( $\pi$-conjugated molecules), a diode (ferrocene alkanethiols) and a switch/memory device (photochromic diarylethenes/organometallic), within a variety of large-area molecular tunneling junctions will be given.

\subsubsection{Top Electrode}

The application or formation of the top electrode has been one of the main bottlenecks in the field of molecular electronics. A variety of techniques are being used to investigate the $J(V)$ characteristics of SAMs within large-area tunneling junctions, however, none of them are ideal, with all techniques having their shortcomings. The most prominent large-area SAM based tunneling junction techniques that have been or are being used to perform $J(V)$ measurements are:

(1) Eutectic Gallium Indium (EGaIn) technique, ${ }^{[18]}$ which consists of an eutectic alloy of Ga and In (EGaIn), which exhibits non-Newtonian properties due to the formation of a thin $\mathrm{Ga}_{2} \mathrm{O}_{3}$ layer on the surface of the EGaIn. These properties allow this material to be shaped into probes or pushed through microchannels, ${ }^{[19]}$ in order to contact the molecular layer and form the top electrode.

(2) PEDOT:PSS technique, ${ }^{[20]}$ which consists of a water-based suspension of conductive polymer(s), poly(3,4-ethylenedioxythiophene) stabilized with poly(4styrenesulphonic acid) (commonly known as PEDOT:PSS), being spin-coated on top of the SAM. A Au layer is then vapor deposited on top of the PEDOT:PSS layer, giving a top electrode of PEDOT:PSS/Au.

(3) Graphene electrode technique, ${ }^{[21]}$ which has been constructed using two similar methods. In the initial study, a multilayer graphene film (mGF) $(<10 \mathrm{~nm}$ thick) was transferred on top of the SAM. ${ }^{[21 \mathrm{a}]}$ In the most recent study, reduced graphene oxide ( $\mathrm{rGO}$ ) was dissolved in DMF, with the supernatant of the rGO solution used to spin coat a rGO film $\left(\sim 10 \mathrm{~nm}\right.$ thick) on top of the SAM. ${ }^{[21 \mathrm{~b}]}$ In 
both cases, a Au layer was vapor-deposited on top of the graphene based layer, giving a top contact of either $\mathrm{mGF} / \mathrm{Au}$ or $\mathrm{rGO} / \mathrm{Au}$.

(4) Mercury (Hg) drop technique, ${ }^{[22]}$ which has been assembled using two different methods. In one method, the junction is formed by bringing two drops of $\mathrm{Hg}$ covered by SAMs, into contact, in a solution containing the molecule of interest, ${ }^{[22 a, 22 b, 23]}$ i.e. $\mathrm{Hg}$ is both the top and bottom electrode. In the other method, the junction is formed by lowering a $\mathrm{Hg}$ drop protruding from a syringe covered by an alkanethiol SAM, into contact with a second SAM (SAM of interest) immobilized on a solid metal surface, i.e. the top electrode consists of $\mathrm{Hg} / \mathrm{SAM}$. This process is carried out in a solution of the alkanethiol SAM used to create the SAM on the Hg drop. ${ }^{[22 d, 23]}$

(5) Metal evaporation technique, ${ }^{[24]}$ which simply consists of vapor deposition of metal onto the SAM, giving a solid metal as the top electrode.

(6) Hybrid technique, which is a combination of two of the techniques listed above, e.g., a conductive polymer spin coated on top of the SAM, which is then addressed by a bare Hg drop. ${ }^{[25]}$

All of the techniques described above, other than the metal evaporated technique, have a protective layer as part of the top electrode. A protective layer is used because evaporating or placing a metal directly onto the SAM has been found to damage and/or penetrate the SAM, leading to a low yield of working junctions and metal filaments dominating charge transport rather than the SAM itself. ${ }^{[24,26]}$ However, each of the protective layers creates illdefined parameters and/or limitations within the tunneling junctions. For EGaIn, the exact thickness, resistivity and surface roughness of the $\mathrm{Ga}_{2} \mathrm{O}_{3}$ layer on the surface of the EGaIn are unknown. ${ }^{[18,27]}$ As for PEDOT:PSS, due to its successful use in tunneling junctions its limitations have been extensively investigated and are as follows. PEDOT:PSS is hygroscopic and hence contains water in ambient conditions leading to a small amount of hysteresis in the $J(V)$ measurements at lower voltages $(<1.0 \mathrm{~V})$ and destruction of the devices at higher voltages $(>2.0 \mathrm{~V}) .{ }^{[28]}$ PEDOT:PSS may influence the $J(V)$ characteristics of temperature dependent measurements, ${ }^{[29]}$ and cause a larger variation of $J$ for junctions with diameters $<5 \mu \mathrm{m} .{ }^{[30]}$ Also, the PEDOT:PSS formulation used must be kept constant as different commercially available PEDOT:PSS formulations have been shown to cause a 14 
variation in the absolute current measured. ${ }^{[29 b, 31]}$ Finally, it is speculated that PEDOT:PSS engulfs the SAM, as PEDOT:PSS tunneling junctions give higher values of $J$ than other tunneling junction techniques. ${ }^{[20]}$ Due to the graphene electrode techniques being recently conceived, their ill-defined parameters and limitations are not well understood. However, in the mGF tunneling junctions, the method used to transfer the solid multi-layer graphene appears cumbersome, ${ }^{[21 a]}$ and in the rGO tunneling junctions, the solvents used to spin coat the rGO layer are difficult/impossible to remove and the homogeneity of the rGO layer itself is ill-defined. ${ }^{[21 b]}$ In the $\mathrm{Hg}$ drop technique, $\mathrm{Hg}$ itself is the major problem rather than the alkanethiol protective layer, as $\mathrm{Hg}$ is toxic, volatile, suffers from electronmigration and easily amalgamates with other metals. ${ }^{[23]}$ Due to these problems, groups such as Whitesides and co-workers have abandoned this technique and now use the EGaIn technique instead. However, the $\mathrm{Hg}$ technique is still used by groups such as Cahen and co-workers who investigate the charge transport of molecular layers formed on semiconductor surfaces. ${ }^{[10 \mathrm{a}, 10 \mathrm{~b}]}$

For the techniques discussed above to be even considered as a potential replacement for, or to be incorporated into, semiconductor technology, they must be stable for years, if not decades at a time, and demonstrate the ability to be integrated into electronic circuits. The only techniques that have been found to be stable for slightly prolonged periods of time (i.e. longer than 30 days) are the PEDOT:PSS technique, ${ }^{[20]}$ and the two graphene electrode techniques, ${ }^{[21]}$ with the PEDOT:PSS technique being the only one able to connect tunneling junctions in series. ${ }^{[30-31]}$ Therefore, it is these techniques that are the closest to commercial applications (albeit still very far from it though) for SAM based devices. However, these techniques require expensive procedures such as photolithography and, therefore, for cheap fundamental laboratory studies on SAMs, the EGaIn technique is also applicable. ${ }^{[18]}$ More detailed explanations about each technique, is given in the reviews by McCreery and coworkers $^{[3,32]}$ and de Boer and co-workers ${ }^{[33]}$ and in Chapter 3.

As each technique has its own shortcomings, there is not one standard technique used to investigate molecular tunneling junctions. Therefore, the discussion below of the charge transport characteristics of each SAM will be segmented into the techniques that were used to investigate it. 


\subsubsection{Molecule-Electrode Interfaces}

The metal-molecule interfaces are highly influential in the charge transport characteristics obtained within molecular tunneling junctions. By varying the type of molecule-metal coupling, from chemisorbed to physisorbed, it is possible to change the conductance measured within a tunneling junction by a few orders of magnitude. ${ }^{[34]}$ This effect is clearly seen when comparing the conductance of an alkanedithiol molecule (which has two chemisorbed contacts) with that of an alkanethiol (which has one chemisorbed contact and one physisorbed contact) when immobilized in between two Au electrodes, as shown experimentally in section 2.3.1.3, Figure $2.7 \mathrm{a},{ }^{[21 \mathrm{~b}]}$ and in section 2.3.1.6, Figure 2.12a. ${ }^{[35]}$ Theoretically this phenomenon can be explained using the Landauer formula, ${ }^{[33-34,36]}$ where the conductance $G$ is given by (Equation 2.1):

$$
G=\frac{2 e^{2}}{h} \times T_{b} \times T_{m o l} \times T_{t}
$$

with $e$ being the elementary charge, $h$ Planck's constant, $T_{b}$ and $T_{t}$ being the interface transmission coefficients of the bottom contact and top contact, respectively, and $T_{m o l}$ being the transmission coefficient of the molecule. From Equation 2.1, it can be seen that if the transmission changes for one of the contacts, such as when the top contact changes from a physisorbed contact to a chemisorbed contact for alkanethiols vs. alkanedithiols, the conductance will change by the same factor.

The energy level alignment of the Fermi level of the metal and the SAMs molecular orbitals can dictate the efficiency of charge transport in tunneling junctions. Misalignment of the energies of the metals Fermi level and the SAMs highest occupied molecular orbital (HOMO) or lowest unoccupied molecular orbital (LUMO) levels can correspond to a tunnel barrier $^{[20,37]}$ which hinders charge transport. However, when the energies are aligned they are energetically accessible to participate in and hence enhance charge transport. ${ }^{[38]}$

\subsection{The Role of SAMs in Charge Transport in Large-Area Molecular Tunneling Junctions}

Ideally, SAMs govern charge transport within molecular tunneling junctions. How they govern the charge transport is dependent on the chemical structure of the molecule within the SAM. Molecules such as alkanethiol/alkanedithiols ${ }^{[20,24,33]}$ and conjugated molecules 
shorter than $\sim 3 \mathrm{~nm}^{[39]}$, force electrons to tunnel through the molecular layer. Tunneling is a temperature independent process. In molecular tunneling junctions, tunneling current $J$ $\left(\mathrm{A} / \mathrm{cm}^{2}\right)$ decays exponentially with the distance between the two electrodes $d(\AA)$ (also known as the barrier width), as approximated by a simple form of the Simmons equation (Equation 2.2 $)^{[37 \mathrm{~b}, 40]}$ where $J_{0}\left(\mathrm{~A} / \mathrm{cm}^{2}\right)$ (pre-exponential factor) is the current density flowing through the electrode-SAM interfaces in the hypothetical case of $d=0 \AA$, and $\beta\left(\AA^{-1}\right)$ is the tunneling decay constant.

$$
J=J_{0} \mathrm{e}^{-\beta \mathrm{d}}
$$

To perform a comprehensive study on SAMs which have electron tunneling as the dominant mechanism of charge transport, $J(V)$ measurements can be performed on SAMs of different lengths, which in turn vary the distance between the two electrodes. By plotting the data as $\log J$ vs. $d$ it is possible to determine $J_{0}$ from the y-intercept and $-\beta\left(\AA^{-1}\right)$ from the slope (Equation 2.2). The slope (- $\beta$ ) quantifies the decay of the tunneling probability with increasing $d$, and it is this term $\beta$ which is the most prominent 'universal parameter' used to evaluate charge transport in molecular systems. Using Equation 2.3, $\beta$ can also be determined, where $\hbar$ is the reduced Planck's constant, $m$ is electron mass, $\Phi_{\mathrm{B}}$ is the barrier height and $\alpha$ is a unitless adjustable parameter used to calculate tunneling through molecules. ${ }^{[7 \mathrm{c}]}$ The terms $\Phi_{\mathrm{B}}$ and $\alpha$ can be obtained from $I(V)$ data fittings.

$$
\beta=\frac{2(2 m)^{1 / 2}}{\hbar} \alpha\left(\Phi_{\mathrm{B}}\right)^{1 / 2}
$$

SAMs of conjugated molecules longer than $\sim 3 \mathrm{~nm}^{[39]}$ and ferrocene alkanethiols ${ }^{[41]}$ can allow electrons to 'hop' across the molecular layer. Electron hopping is a temperature dependent process and therefore this charge transport mechanism can be investigated by performing temperature dependent $J(V)$ measurements. When electron hopping is the dominant mechanism of charge transport, the value of $J$ measured decreases with decreasing temperature, whereas when tunneling is the dominant mechanism of transport, $J$ stays constant with temperature change. ${ }^{[41 \mathrm{~b}]}$

In the remainder of section 2.3, the charge transport characteristics of alkanethiols/alkanedithiols (section 2.3.1), $\pi$-conjugated molecules (section 2.3.2), ferrocene alkanethiols (section 2.3.3), organometallic SAMs (section 2.3.4) and photo-induced electrical switches (section 2.3.5) in a variety of tunneling junction architectures will be 
discussed. Where possible, the experiments performed to determine the charge transport mechanism will be described, along with the values obtained for universal charge transport parameters such as $\beta$.

\subsubsection{Alkanethiols and Alkanedithiols}

Due to their relatively simple chemical structure, alkanethiols and alkanedithiols are the traditionally used molecules when evaluating the ability of new and novel molecular tunneling junction techniques to perform reproducible and reliable $J(V)$ measurements (Figure 2.4).$^{[18,20-21]}$ They consist simply of an aliphatic carbon chain and either a single terminal thiol moiety, or two terminal thiol moieties, and readily self-assemble into arrays of well-ordered, highly dense SAMs on metal surfaces. Due to the large HOMO - LUMO gap of the carbon chain and thus poor electronic conductivity, alkanethiol/alkanedithiol SAMs act as a dielectric layer in between the two electrodes in molecular tunneling junctions, forcing the electrons to tunnel through the molecular layer. To evaluate a new molecular tunneling junction technique, $J(V)$ measurements can be performed on SAMs of alkanethiols/alkanedithiols with a carbon chain of different lengths, which in turn varies the distance between the two electrodes. Published $\beta$ values for alkanethiols and alkanedithiols in single-molecule and large-area self-assembled junctions range from $0.38-0.88 \AA^{-1}$. $^{[33]}$ The molecular structures of the molecules discussed in this section (2.3.1) are given on the next page (page 19). 


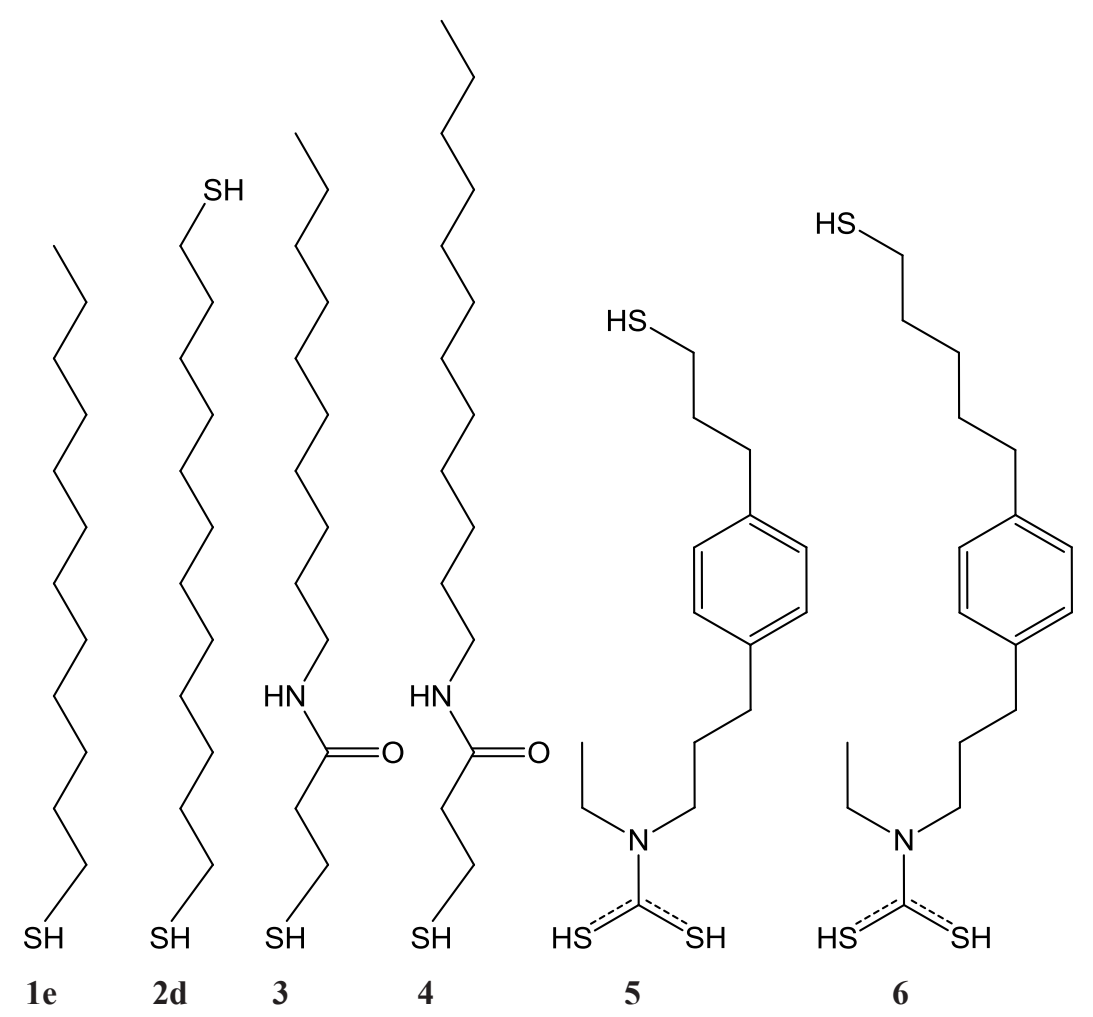

Figure 2.4: Molecular structure of an alkanethiol (1) (1-dodecanethiol (1e)), an alkanedithiol (2) (1,12dodecanedithiol (2d)), n-decane-3-thiopropanamide (3), n-dodecane-3-thiopropanamide (4), ethyl(3-(4-(3mercaptopropyl)phenyl)propyl)carbamodithioic acid (5), and ethyl(3-(4- $(5-$ mercaptopentyl)phenyl)propyl)carbamodithioic acid (6). 


\subsubsection{EGaIn Tunneling Junctions}

Whitesides and co-workers, have reported various studies of charge transport through SAMs of alkanethiols in EGaIn tunneling junctions. ${ }^{[18,42]}$ In the most recent study, ${ }^{[42]}$ statistically relevant numbers of $J(V)$ measurements were carried out $\pm 0.5 \mathrm{~V}$ across SAMs of alkanethiols (1), containing both odd and even numbers of carbon atoms $\left(\left(\mathrm{C}_{n} \mathrm{H}_{2 n+1} \mathrm{SH}\right)\right.$, where $n=9-18$, and for simplicity, $\mathrm{C}_{n} \mathrm{H}_{2 n+1} \mathrm{SH}$ will be indicated as $\mathrm{C}_{n} \mathrm{SH}$ throughout this thesis), with a yield of working junctions of $\sim 80 \%$. The authors demonstrated that evennumbered alkanethiols (1) gave higher values of $J$ than odd-numbered alkanethiols (1). Therefore, the typical exponential decrease of $J$ was not observed with the increase in molecular length. However, when the authors analyzed the $J(V)$ data obtained for the evennumbered alkanethiols and odd-numbered alkanethiols separately, both individual sets of data displayed that $J$ decreased exponentially with increasing molecular length. Therefore, separate values of $\beta$ were given for the even-numbered alkanethiols and the odd-numbered alkanethiols, with $\beta_{\text {even }}=1.04 \pm 0.06 n_{\mathrm{c}}^{-1}\left(\sim 0.81 \AA^{-1}\right)$ and $\beta_{\text {odd }}=1.19 \pm 0.08 n_{\mathrm{c}}^{-1}\left(\sim 0.92 \AA^{-1}\right)$. Interestingly, within this study the authors also stated that when measuring alkanethiols longer than $\mathrm{C}_{18} \mathrm{SH}$ (1h) and shorter than $\mathrm{C}_{9} \mathrm{SH}$ (1c) they obtained inconsistent results, indicating the length limitations of measuring alkanethiols within EGaIn tunneling junctions.

a)

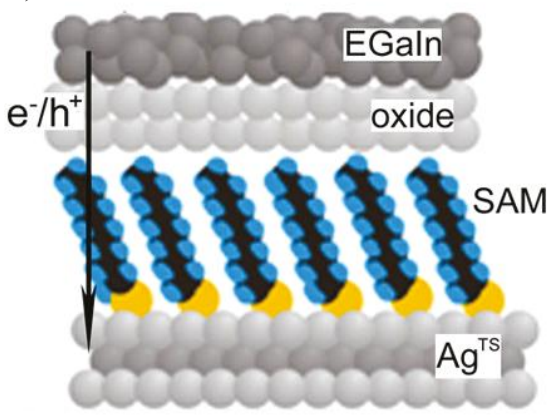

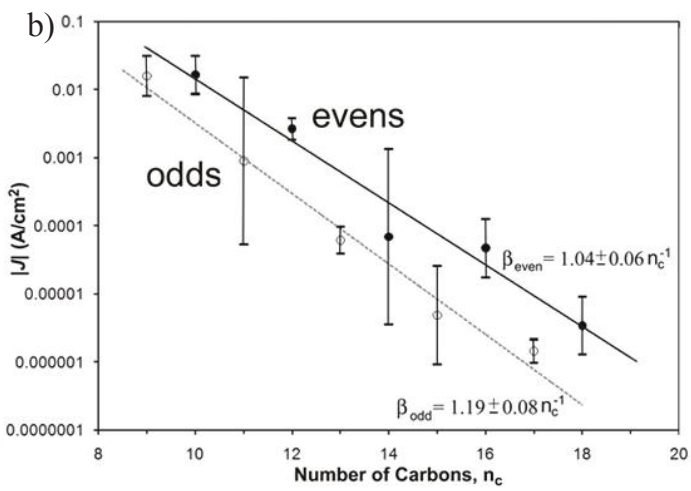

Figure 2.5: (a) Idealized schematic of an alkanethiol EGaIn tunneling junction, with an alkanethiol (1) SAM immobilized on a $\mathrm{Ag}^{\mathrm{TS}}$ surface (the bottom electrode), with the $\left(\mathrm{Ga}_{2} \mathrm{O}_{3}\right)$ EGaIn top electrode. (b) Semi-log plot of the averaged values of $J$ measured in EGaIn tunneling junctions versus the number of carbon atoms for SAMs of $\mathrm{C}_{9} \mathrm{SH}-\mathrm{C}_{19} \mathrm{SH}$ (Both Figures are reprinted with permission from. ${ }^{[42]}$ Copyright (C) 2011 American Chemical Society). 


\subsubsection{PEDOT:PSS Tunneling Junctions}

In an important study, ${ }^{[20]}$ de Boer and co-workers, investigated charge transport through SAMs of alkanedithiols (2) $\left(\left(\mathrm{HS}\left(\mathrm{C}_{n} \mathrm{H}_{2 n}\right) \mathrm{SH}\right)\right.$ and for simplicity $\mathrm{HS}\left(\mathrm{C}_{n} \mathrm{H}_{2 n}\right) \mathrm{SH}$ will be indicated as $\left.\mathrm{HS}\left(\mathrm{C}_{\mathrm{n}}\right) \mathrm{SH}\right)$ throughout this thesis) in PEDOT:PSS tunneling junctions. $J(V)$ measurements were carried out $\pm 0.75 \mathrm{~V}$ on the alkanedithiols (2), 1,8-octanedithiol $\left(\mathrm{HSC}_{8} \mathrm{SH}\right)(\mathbf{2 a}), 1,10$-decanedithiol $\left(\mathrm{HSC}_{10} \mathrm{SH}\right)(\mathbf{2} \mathbf{c}), 1,12$-dodecanedithiol $\left(\mathrm{HSC}_{12} \mathrm{SH}\right)$ (2d) and 1,14-tetradecanedithiol ( $\left.\mathrm{HSC}_{14} \mathrm{SH}\right)$ (2e) (Figure 2.6a). A minimum of 17 devices were measured for each alkanedithiol, with a yield of 'working junctions' of $>95 \%$. The authors found that $J$ decreased exponentially with increasing alkanedithiol length, demonstrating that through-bond electron tunneling is the mechanism of charge transport. (inset Figure 2.6a). They determined $\beta$ to be $0.66 \pm 0.06,0.61 \pm 0.05$ and $0.57 \pm 0.05 \AA^{-1}$ at a bias of 0.1 , 0.3 and $0.5 \mathrm{~V}$, respectively.

In an extended study, ${ }^{[43]}$ de Boer and co-workers, measured the $J(V)$ characteristics of SAMs of longer alkanedithiols, $\mathrm{HSC}_{14} \mathrm{SH}(\mathbf{2 e})$ and 1,16-hexadecanedithiol $\left(\mathrm{HSC}_{16} \mathrm{SH}\right)(\mathbf{2 f})$. In this study, they found that using $3 \mathrm{mM}$ solutions to form long alkanedithiol SAMs (the standard concentration used to form the SAMs in the previous study ${ }^{[20]}$ ) caused $\mathrm{HSC}_{14} \mathrm{SH}(\mathbf{2 e})$ to produce slightly asymmetric $J(V)$ curves (as could already be seen in the previous study ${ }^{[20]}$ ), and $\mathrm{HSC}_{16} \mathrm{SH}(\mathbf{2 f})$ to exhibit a higher value of $J$ than $\mathrm{HSC}_{14} \mathrm{SH}(\mathbf{2 e})$ (which in theory should not occur as $\mathrm{C}_{16}$ is a thicker insulating layer than $\mathrm{C}_{14}$ ). The authors attributed this to the longer carbon chains of these alkanethiols being able to loop/backbend over themselves, which they described as a 'looped phase'. ${ }^{[43]}$ This causes thinner layers of SAMs to be formed, allowing the electrons to tunnel through a shorter distance. By decreasing or increasing the concentration of $\mathrm{HSC}_{14} \mathrm{SH}(\mathbf{2 e})$ in solution by 100 times $(0.3 \mathrm{mM}$ to $30 \mathrm{mM})$, the authors found that it was possible to change the value of $J$ obtained for the tunneling junctions. Lower concentrations $(0.3 \mathrm{mM})$ of $\mathrm{HSC}_{14} \mathrm{SH}(\mathbf{2 e})$ gave higher values of $J$, as a larger number of the molecules were in the 'looped phase' (producing a thinner molecular layer), whereas higher concentrations gave lower values of $J$, as almost all of alkanedithiols were in the 'standing phase', thus producing highly ordered, densely packed monolayers, i.e., forming a thicker and denser molecular layer for the electrons to tunnel through. SAMs of $\left(\mathrm{HSC}_{16} \mathrm{SH}\right)$ (2f) formed in $30 \mathrm{mM}$ solutions were also found to produce thicker and more densely packed monolayers than in $3 \mathrm{mM}$ solutions (inset Figure 2.6b). Figure 2.6b displays the exponential decrease of $J$ with increasing molecular length, with values of $J$ obtained 
from $\mathrm{HSC}_{14} \mathrm{SH}(\mathbf{2 e})$ and $\mathrm{HSC}_{16} \mathrm{SH}(\mathbf{2 f})$ formed in $30 \mathrm{mM}$ concentrated solutions and values of $J$ obtained from $\mathrm{HSC}_{8} \mathrm{SH}$ (2a), $\mathrm{HSC}_{10} \mathrm{SH}(\mathbf{2}), \mathrm{HSC}_{12} \mathrm{SH}(\mathbf{2 d})$, and $\mathrm{HSC}_{14} \mathrm{SH}(\mathbf{2 e})$ formed in $3 \mathrm{mM}$ solutions. The exponential decrease of $J$ with increasing molecular length demonstrates that only the conductance through long alkanedithiol SAMs formed in $30 \mathrm{mM}$ solutions, (which allows for almost all of the alkanedithiols to be in the full 'standing-up' phase), is consistent with the through-bond electron tunneling seen for the shorter alkanedithiols formed in $3 \mathrm{mM}$ solutions.

a)

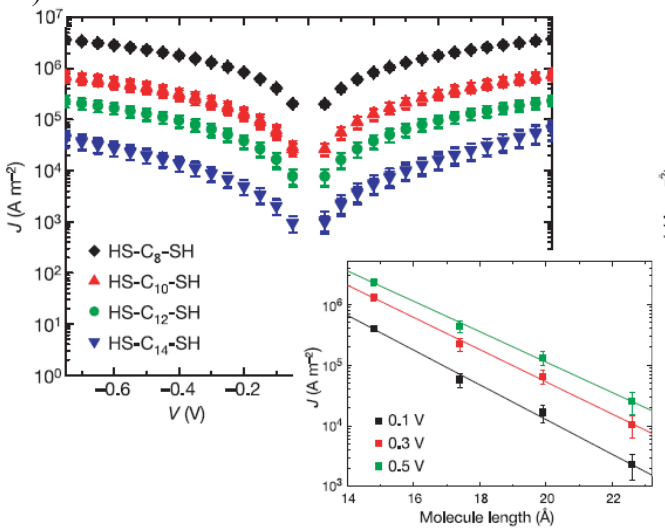

b)

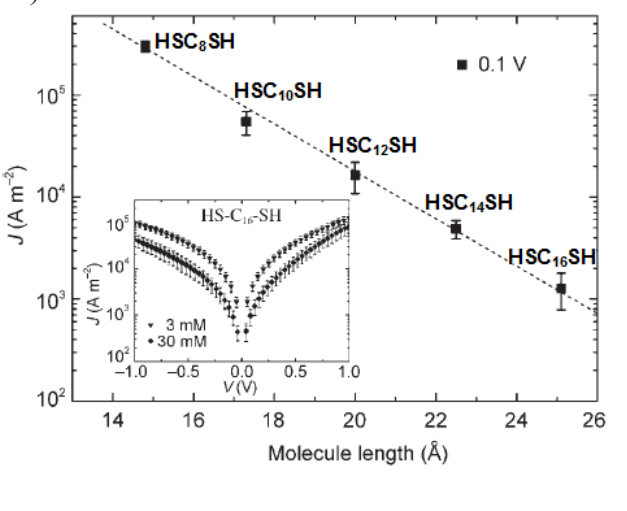

Figure 2.6: (a) Semi-log plot of the averaged $J(V)$ measurements performed $\pm 0.75 \mathrm{~V}$ across the SAMs of $\mathrm{HSC}_{8} \mathrm{SH}$ (2a), $\mathrm{HS}_{10} 0_{10} \mathrm{SH}(\mathbf{2 c}), \mathrm{HSC}_{12} \mathrm{SH}(\mathbf{2 d})$ and $\mathrm{HSC}_{14} \mathrm{SH}(\mathbf{2 e})$. The inset displays a semi-log plot of the $J$ as a function of molecular length at different biases. The exponential decrease in $J$ for increasing molecular length demonstrates that the mechanism of charge transport is through-bond tunneling. (Figure reprinted by permission from Macmillan Publishers Ltd: Nature, ${ }^{[20]}$ Copyright (C) 2006). (b) A Semi-log plot of $J$ measured for $\mathrm{SAMs}_{\mathrm{ASC}} \mathrm{HSC}_{14} \mathrm{SH}(\mathbf{2 e})$ and $\mathrm{HSC}_{16} \mathrm{SH}(\mathbf{2 f})$ formed in $30 \mathrm{mM}$ solutions and SAMs of $\mathrm{HSC}_{8} \mathrm{SH}(\mathbf{2 a})$, $\mathrm{HS}_{10}{ }_{10} \mathrm{SH}(\mathbf{2 c}), \mathrm{HSC}_{12} \mathrm{SH}(\mathbf{2 d})$ formed in $3 \mathrm{mM}$ solutions. The exponential decrease of $J$ with increasing molecular length demonstrates that almost all of the long alkanethiols are in the 'standing up phase' allowing for through-bond tunneling. Inset displays the difference in $J$ measured when SAMs of $\mathrm{HSC}_{16} \mathrm{SH}(\mathbf{2 f})$ are formed in either a $3 \mathrm{mM}$ or $30 \mathrm{mM}$ solution. (Figure adapted with permission from. ${ }^{[43]}$ Copyright (C) 2007 John Wiley and Sons).

De Leeuw and co-workers investigated the charge transport characteristics of alkanethiol (1) SAMs in PEDOT:PSS tunneling junctions. ${ }^{[30-31,44]}$ The authors performed statistically relevant numbers of $J(V)$ measurements on alkanethiols (1) with an even number of carbon atoms $\left(\left(\mathrm{C}_{\mathrm{n}} \mathrm{SH}\right)\right.$ where $\left.\mathrm{n}=8-22\right)$. Compared to the alkanedithiol SAMs investigated in the 
PEDOT:PSS tunneling junctions, the yield, reproducibility, stability and area scaling of the alkanethiols were found to be identical. For alkanethiols where $\mathrm{n}=8-12$, the authors found that the normalized resistance $(R S)$ was indistinguishable from PEDOT:PSS itself. However, for alkanethiols where $\mathrm{n}=14-22, R S$ was found to increase exponentially with molecular length (in other words $J$ decreased exponentially with molecular length), with $\beta 0.73 \AA^{-1}$.

\subsubsection{Graphene Tunneling Junctions}

In multilayer graphene film (mGF) tunneling junctions, Lee and co-workers, investigated the $J(V)$ characteristics of SAMs of 1-octanethiol $\left(\mathrm{C}_{8} \mathrm{SH}\right)(\mathbf{1 b}), 1$-dodecanethiol $\left(\mathrm{C}_{12} \mathrm{SH}\right)(\mathbf{1 e})$, 1-hexadecanethiol $\left(\mathrm{C}_{16} \mathrm{SH}\right)(\mathbf{1 g})$ and 1,8-octanedithiol $\left(\mathrm{HSC}_{8} \mathrm{SH}\right)(\mathbf{2 a}){ }^{[21 \mathrm{a}]}$ The authors performed a statistically relevant number of $J(V)$ measurements $\pm 1.5 \mathrm{~V}$, with a yield of working devices of $\sim 90 \%$ (Figure $2.7 \mathrm{a}$ ). They found that $J$ decreased exponentially with the increase in length of the alkanethiol (Figure 2.7b). This phenomenon along with temperature independent $J(V)$ characteristics, confirmed that electron tunneling was the dominant mechanism of charge transport. The authors determined $\beta$ to be $0.85 \pm 0.11 \AA^{-1}$. Upon comparing the $J(V)$ characteristics of $\mathrm{C}_{8} \mathrm{SH}(\mathbf{1 b})$ and $\mathrm{HSC}_{8} \mathrm{SH}$ (2a) (monothiol vs. dithiol), the authors found that $\mathrm{C}_{8} \mathrm{SH}(\mathbf{1 b})$ exhibited higher values of $J$. This is because in the mGF tunneling junctions, $\mathrm{HSC}_{8} \mathrm{SH}(\mathbf{2 a})$ is unable to form the second chemisorbed contact with the graphene layer, which would typically be possible if the top contact was only a metal, leaving $\mathrm{C}_{8} \mathrm{SH}(\mathbf{1 b})$ to only form a physisorbed contact. Therefore, in the mGF tunneling junctions both $\mathrm{C}_{8} \mathrm{SH}(\mathbf{1 b})$ and $\mathrm{HSC}_{8} \mathrm{SH}$ (2a) can only form a physisorbed contact with the graphene layer, thus making $J$ obtained predominantly dependent on their molecular length. As $\mathrm{C}_{8} \mathrm{SH}(\mathbf{1 b})$ is shorter than $\mathrm{HSC}_{8} \mathrm{SH}(\mathbf{2 a}), \mathrm{C}_{8} \mathrm{SH}(\mathbf{1 b})$ exhibits higher values of $J$.

In reduced graphene oxide (rGO) tunneling junctions, Lee and co-workers, investigated the $J(V)$ characteristics of SAMs of $\mathrm{C}_{8} \mathrm{SH}(\mathbf{1 b}), 1$-decanethiol $\left(\mathrm{C}_{10} \mathrm{SH}\right)(\mathbf{1 d})$, and $\left(\mathrm{C}_{12} \mathrm{SH}\right)(\mathbf{1 e})$ (Figure 2.7c). ${ }^{[21 \mathrm{~b}]}$ Working devices were found with a yield of $99 \%$ and, as in the $\mathrm{mGF}$ tunneling junctions, $J$ decreased exponentially with increasing molecular length, with $\beta 0.82$ $\pm 0.12 \AA^{-1}$. 
a)

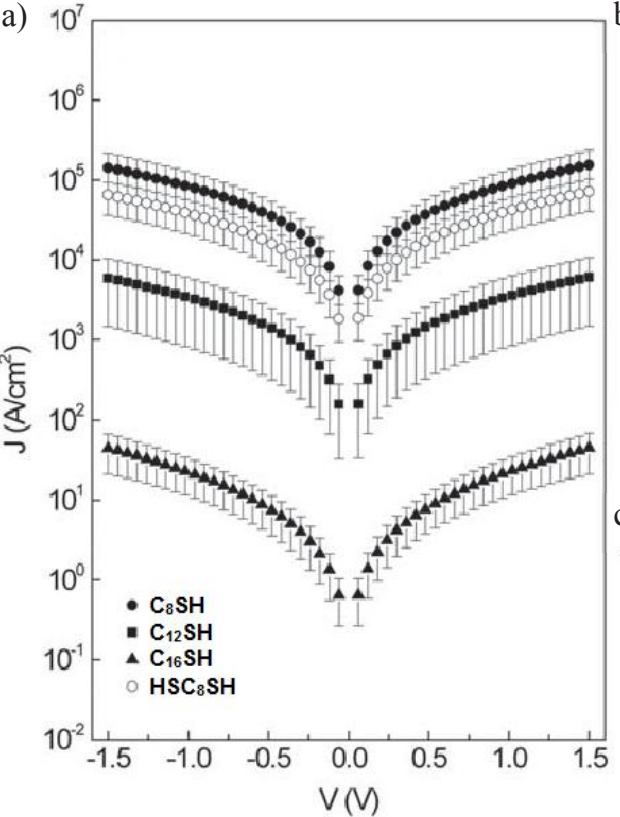

b)
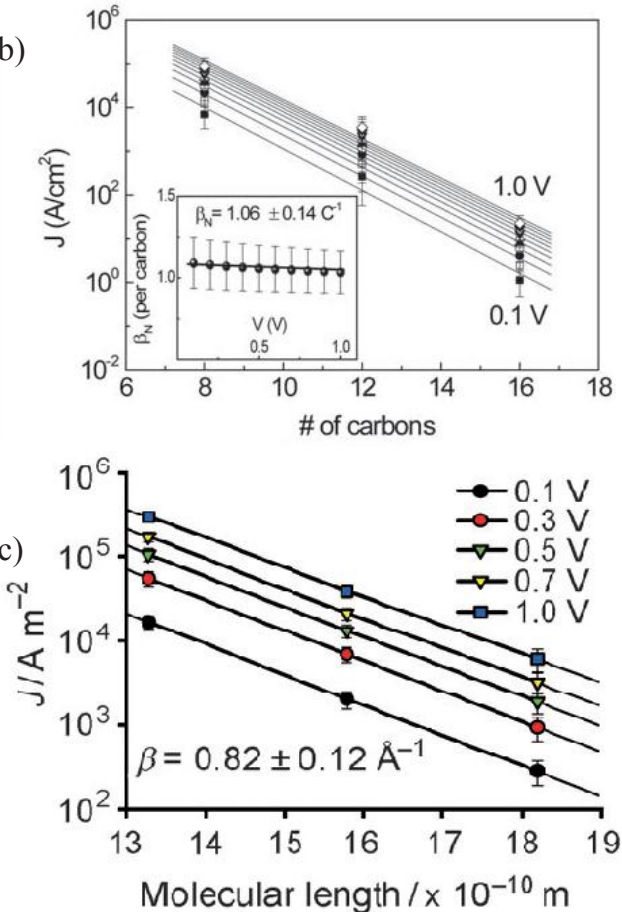

Figure 2.7: (a) Semi-log plot of the averaged $J(V)$ data measured $\pm 1.5 \mathrm{~V}$, for $\mathrm{C}_{8} \mathrm{SH}(\mathbf{1 b}), \mathrm{C}_{12} \mathrm{SH}(\mathbf{1 e}), \mathrm{C}_{16} \mathrm{SH}$ (1g) and $\mathrm{HSC}_{8} \mathrm{SH}$ (2a) in mGF tunneling junctions. (b) Semi-log plot of the averaged values of $J$ measured at different biases in mGF tunneling junctions as a function of the number of carbon atoms in the SAMs of, $\mathrm{C}_{8} \mathrm{SH}$ (1b), $\mathrm{C}_{12} \mathrm{SH}(\mathbf{1 e})$, and $\mathrm{C}_{16} \mathrm{SH}(\mathbf{1 g})$. (c) Semi-log plot of the averaged values of $J$ measured at different biases in rGO tunneling junctions versus molecular length of the $\mathrm{SAMs} \mathrm{C}_{8} \mathrm{SH}(\mathbf{1 b}), \mathrm{C}_{10} \mathrm{SH}(\mathbf{1 d})$, and $\mathrm{C}_{12} \mathrm{SH}(\mathbf{1 e})$. The exponential decrease in $J$ seen in (b) and (c) demonstrates that the mechanism of charge transport is through-

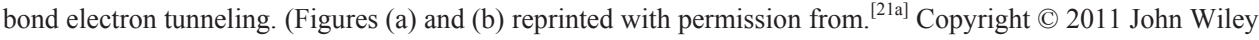
and Sons. Figure (c) reprinted with permission from. ${ }^{[2 \mathrm{~b}]}$ Copyright (C) 2010 John Wiley and Sons).

\subsubsection{Mercury Drop Tunneling Junctions}

A variety of $J(V)$ measurements on SAMs of alkanethiols (1) have been performed in two different mercury drop junction setups. Whitesides and co-workers, formed their alkanethiol SAMs predominantly on a solid metal surface, ${ }^{[22 d, 23,45]}$ whereas Majda and Slowinski and co-workers, formed their SAMs on only a Hg surface. ${ }^{[22 c, 22 e, 46]}$ Both groups contact the 
SAM in the same manner, using a Hg top electrode coated with an alkanethiol SAM as the protective layer.

The work carried out by Whitesides and co-workers, produced junctions with a yield of $\sim 25 \%$. They determined $\beta$ for the alkanethiols to be $0.87 \pm 0.1 \AA^{-1}$ on vapor-deposited $\mathrm{Ag}$ surfaces (Figure 2.8a), ${ }^{[45]}$ and $0.57 \AA^{-1}$ or $0.64 \AA^{-1}$ (depending on the statistical analysis used) on template stripped Ag surfaces. ${ }^{[13 b]}$ These authors also carried out an extensive study on the breakdown voltages (BDVs) of alkanethiols of different lengths exposed to different conditions. ${ }^{[22 \mathrm{~d}]}$ They reported that the BDV showed an approximately linear dependence on the alkanethiol chain lengths from $\mathrm{C}_{7} \mathrm{SH}$ (1a) to $\mathrm{C}_{16} \mathrm{SH}(\mathbf{1 g})$, with an increase of $\sim 0.3 \mathrm{~V}$ per $\mathrm{CH}_{2}$ group, which then increased more slowly $\left(\sim 0.2 \mathrm{~V}\right.$ per $\mathrm{CH}_{2}$ group) for alkanethiols of $\mathrm{C}_{16} \mathrm{SH}$ (1g) to $\mathrm{C}_{26} \mathrm{SH}$ (1i). They determined that the BDV of the alkanethiol changes when being formed on different metals, due to the packing and tilt angle of the SAM being predetermined by the metals properties. The BDV values increased in the order $\mathrm{Au}<\mathrm{Hg} \approx \mathrm{Cu} \approx \mathrm{Ag}$ (Figure 2.8b). The BDV values also decreased if the surfaces of the metals became rougher. However, the BDVs were independent of the solvent used to dissolve the alkanethiol and form the junction in, unless the alkanethiol was insoluble in the solvent, which caused the BDV to decrease. Interestingly, the authors also reported that the electric field applied across the junction increased linearly for shorter alkanethiols $\mathrm{C}_{7} \mathrm{SH}$ (1a) $-\mathrm{C}_{14} \mathrm{SH}$ (1f), and then became constant after $\mathrm{C}_{14} \mathrm{SH}$ (1f).

In an extended study Whitesides and co-workers, ${ }^{[45 b]}$ varied the terminal functional moiety of the alkanethiol SAMs in order to study the effect that different bond interactions have on charge transport. The ability of the bond interactions to facilitate electron tunneling, in descending order are covalent $>$ hydrogen $>$ van der Waals contacts. 
a)

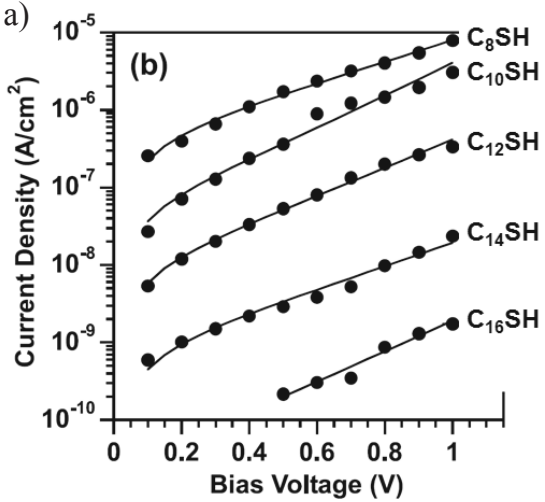

b)

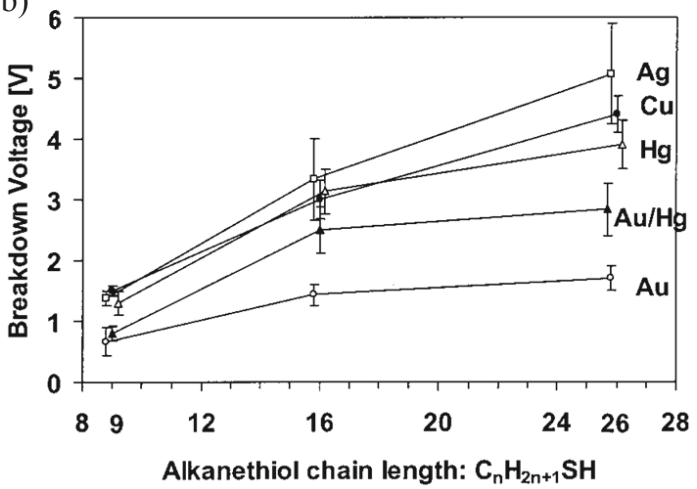

Figure 2.8: (a) Semi-log plot of the averaged $J(V)$ data measured $0 \rightarrow 1.0 \mathrm{~V}$ for SAMs of $\mathrm{C}_{8} \mathrm{SH}(\mathbf{1 b}), \mathrm{C}_{10} \mathrm{SH}$ (1d), $\mathrm{C}_{12} \mathrm{SH}$ (1e), $\mathrm{C}_{14} \mathrm{SH}$ (1f), $\mathrm{C}_{16} \mathrm{SH}$ (1g) formed on a vapor-deposited $\mathrm{Ag}$ surface in $\mathrm{Hg} / \mathrm{C}_{16} \mathrm{SH}$ tunneling junctions (Figure adapted from, ${ }^{[23]}$ Copyright $\mathbb{C}$ 2002, with permission from Elsevier). (b) Plot displaying the averaged breakdown voltage in $\mathrm{Hg} / \mathrm{C}_{16} \mathrm{SH}$ tunneling junctions as a function of alkanethiol chain length, for alkanethiol SAMs formed on the metals $\mathrm{Ag}, \mathrm{Cu}, \mathrm{Hg}, \mathrm{Au} / \mathrm{Hg}$ and $\mathrm{Au}$ (Figure reprinted with permission from. ${ }^{[22 d]}$ Copyright $(1999$ American Chemical Society).

Majda and co-workers performed $J(V)$ measurements $\pm 1.5 \mathrm{~V}$ across SAMs of alkanethiols. As in all successful $J(V)$ measurements of alkanethiol SAMs, they reported that the current measured decreased exponentially with an increase of junction thickness, indicating that electron tunneling is the dominant mechanism of charge transport and reported $\beta$ to be $0.89 \pm 0.10$ per $\mathrm{CH}_{2}\left(\sim 0.68 \AA^{-1}\right)$ (Figure 2.9b). ${ }^{[22 c]}$ The $J(V)$ characteristics of SAMs of $\mathrm{n}$-decane- and n-dodecane-3-thiopropanamides ((3) and (4) respectively) (Figure 2.9a), which only differ to alkanethiol SAMs through the inclusion of an amide group along the carbon backbone, were also investigated. These SAMs were found to give significantly higher tunneling currents than alkanethiols with the same number of carbon atoms (Figure $2.9 \mathrm{~b})$. The authors suggest that this is due to the lateral hydrogen bonding between the amide groups resulting in an increase in strength of the electronic coupling. 
a)

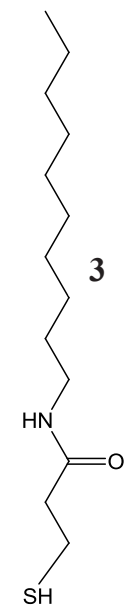

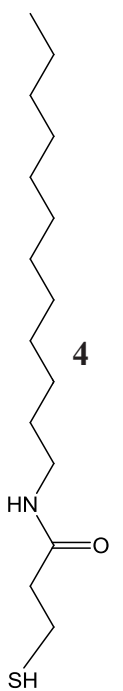

b)

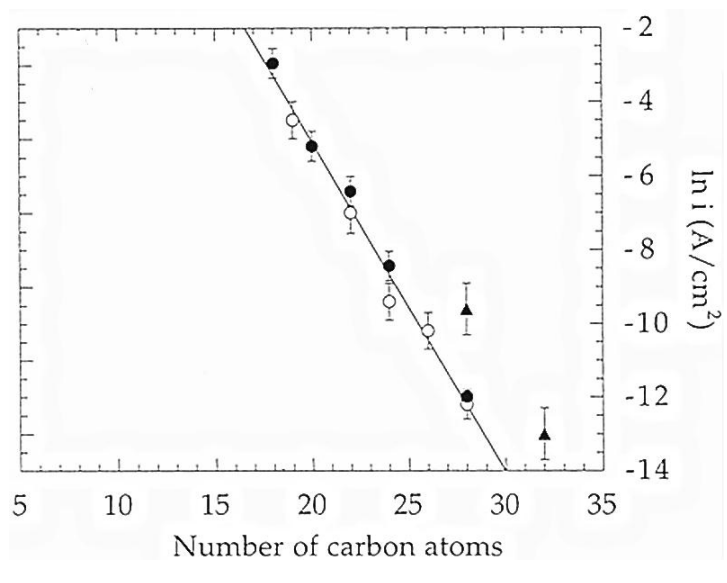

Figure 2.9: (a) Molecular structure of n-decane-3-thiopropanamide (3) and n-dodecane-3-thiopropanamide (4). (b) Semi-log plot of $I$ vs. number of carbon atoms for junctions of symmetric alkanethiol bilayers (black circles), asymmetric alkanethiol bilayers $\left(\mathrm{Hg}-\mathrm{C}_{\mathrm{n}}-\mathrm{C}_{\mathrm{m}}-\mathrm{Hg}\right.$, where the total number of carbons $=\mathrm{n}+\mathrm{m}$ ), with $\mathrm{n}: \mathrm{m}$ $=9: 10,10: 12,10: 14,12: 14,12: 16$ (open circles) and the symmetric SAMs of $\mathrm{n}$-decane-3-thiopropanamide (3) and n-dodecane-3- thiopropanamide (4), where the number of atoms include the nitrogen atom of the amide group (black triangles) (Figure (b) adapted with permission from. ${ }^{[22 c]}$ Copyright $\subset 1999$ American Chemical Society).

\subsubsection{Hybrid Tunneling Junctions}

Rampi and co-workers investigated the $J(V)$ characteristics of alkanethiol SAMs in hybrid tunneling junctions, where the top contact was applied by spin-coating the undoped polymer of poly[( $m$-phenylenevinylene)-co-(2,5-dioctoxy- $p$-phenylenevinylene) $(\mathrm{PmPV})$, which was then contacted by a mercury drop. ${ }^{[25]}$ The authors performed $J(V)$ measurements $\pm 0.5 \mathrm{~V}$ across SAMs of 1-octanethiol $\left(\mathrm{C}_{8} \mathrm{SH}\right)(\mathbf{1 b}), 1$-decanethiol $\left(\mathrm{C}_{10} \mathrm{SH}\right)(\mathbf{1 d})$, 1-dodecanethiol $\left(\mathrm{C}_{12} \mathrm{SH}\right)(\mathbf{1 e})$, 1-tetradecanethiol $\left(\mathrm{C}_{14} \mathrm{SH}\right)(\mathbf{1 f})$ and 1-hexadecanethiol $\left(\mathrm{C}_{16} \mathrm{SH}\right)(\mathbf{1 g})$ (Figure 2.10a). They found that $J$ decreased exponentially with increasing molecular length with $\beta 0.90 \pm 0.03 \AA^{-1}$ (Figure 2.10b). The $J(V)$ data presented for the alkanethiol SAMs does produce a clear trend, however, it is unclear if this $J(V)$ data is an average of statistically relevant numbers of $J(V)$ data, as no yields and minimal error bars are given. 

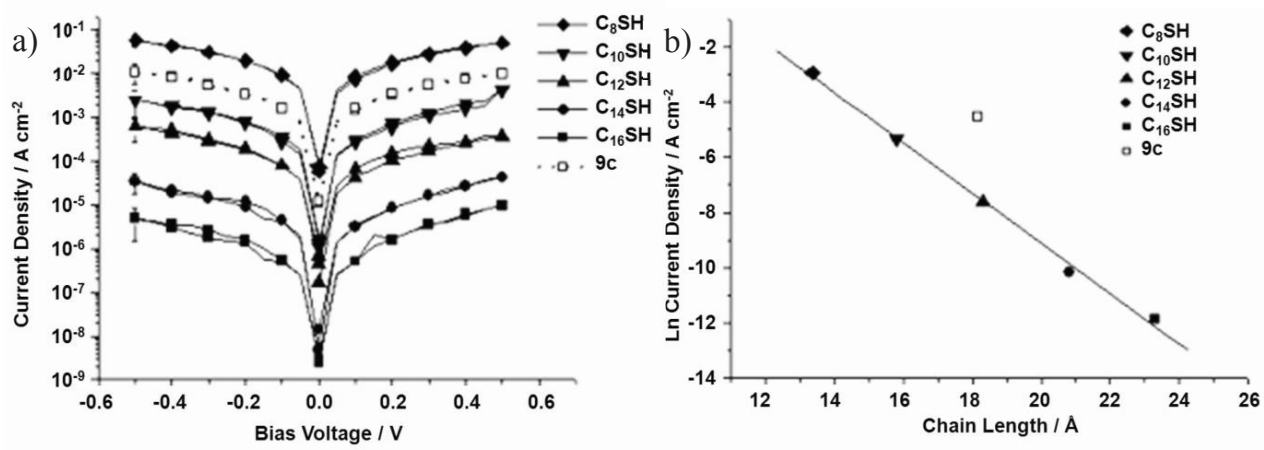

Figure 2.10: (a) Semi-log plot of $J(V)$ measurements performed on SAMs of 1-octanethiol $\left(\mathrm{C}_{8} \mathrm{SH}\right)(\mathbf{1 b})$, 1-decanethiol $\left(\mathrm{C}_{10} \mathrm{SH}\right)(\mathbf{1 d})$, 1-dodecanethiol $\left(\mathrm{C}_{12} \mathrm{SH}\right)(\mathbf{1 e})$, 1-tetradecanethiol $\left(\mathrm{C}_{14} \mathrm{SH}\right)(\mathbf{1 f})$, 1-hexadecanethiol $\left(\mathrm{C}_{16} \mathrm{SH}\right)(\mathbf{1 g})$ and ([1,1':4'1"'-terphenyl]-4-thiol (9c) discussed later in section 2.3.2.4.). (b) Semi-log plot of the averaged values of $J$ in $\mathrm{PmPV} / \mathrm{Hg}$ tunneling junctions as a function of the molecular length of the SAMs of 1-octanethiol $\left(\mathrm{C}_{8} \mathrm{SH}\right)(\mathbf{1 b})$, 1-decanethiol $\left(\mathrm{C}_{10} \mathrm{SH}\right)(\mathbf{1 d})$, 1-dodecanethiol $\left(\mathrm{C}_{12} \mathrm{SH}\right)$ (1e), 1-tetradecanethiol $\left(\mathrm{C}_{14} \mathrm{SH}\right)(\mathbf{1 f})$, 1-hexadecanethiol $\left(\mathrm{C}_{16} \mathrm{SH}\right)(\mathbf{1 g})$ and [1,1':4'1"'-terphenyl]-4-thiol (9c) discussed later in section 2.3.2.4 (Figures (a) and (b) reprinted with permission from. ${ }^{[25]}$ Copyright (C) 2007 John Wiley and Sons).

Wrochem and co-workers investigated the $J(V)$ characteristics of alkanethiol and dithiocarbamate SAMs in hybrid tunneling junctions, where the top contact was applied by spin-coating PEDOT:PSS on top of the SAM, which was then contacted by a mercury drop. $^{[17 \mathrm{a}]}$ The authors performed $J(V)$ measurements $\pm 1.0 \mathrm{~V}$ across SAMs of 1-octanedithiol $\left(\mathrm{HSC}_{8} \mathrm{SH}\right) \quad$ (2a), 1-dodecanedithiol $\quad\left(\mathrm{HSC}_{12} \mathrm{SH}\right) \quad$ (2d), ethyl(3-(4-(3mercaptopropyl)phenyl)propyl)carbamodithioic acid (5), and ethyl(3-(4-(5mercaptopentyl)phenyl)propyl)carbamodithioic acid (6) (Figure 2.11). From the two alkanethiol SAMs measured, the authors determined that $J$ decreased exponentially with increasing molecular length with $\beta \sim 0.5 \AA^{-1}$. The values of $J$ for 5 and $\mathbf{6}$ fall between that of $\mathrm{HSC}_{8} \mathrm{SH}(\mathbf{2 a})$ and $\mathrm{HSC}_{12} \mathrm{SH}(\mathbf{2 d})$, with 5 showing higher values of $J$ than $\mathbf{6}$ due to its shorter carbon chain. The $J(V)$ curves of $\mathbf{5}$ and $\mathbf{6}$ also show a very slight asymmetry, rectifying slightly at a positive bias (rectification ratio of 1.32 at $\pm 1.0 \mathrm{~V}$ ) (Figure $2.11 \mathrm{~b}$ ). The authors attribute these phenomena to the phenyl ring in these SAMs. However, the rectification is so negligible, that the origin of these phenomena is unclear. True rectifying molecules will be discussed in detail in section 2.3.3. 
<smiles>CCN(CCCc1ccc(CCCS)cc1)C(=S)S</smiles>

5<smiles>CCN(CCCc1ccc(CCCCCS)cc1)C(=S)S</smiles>

b)

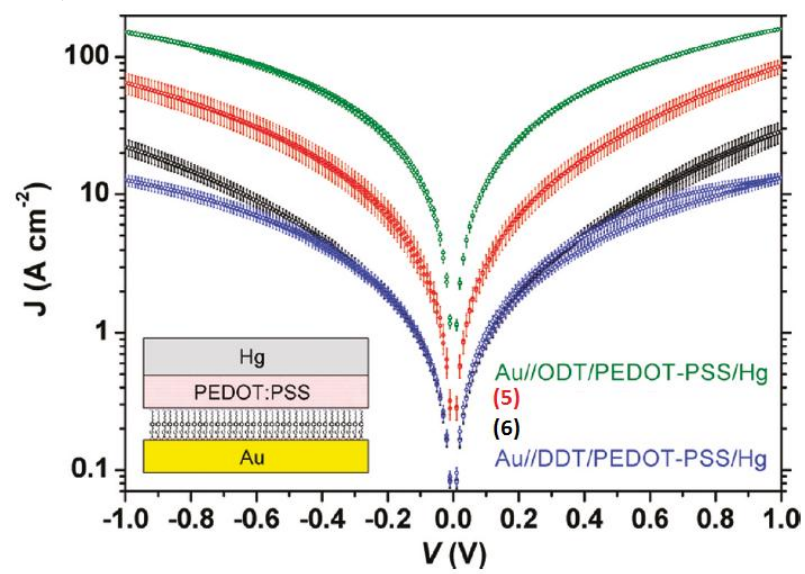

Figure 2.11: (a) Molecular structures of ethyl(3-(4-(3-mercaptopropyl)phenyl)propyl)carbamodithioic acid (5), and ethyl(3-(4-(5-mercaptopentyl)phenyl)propyl)carbamodithioic acid (6). (b) Semi-log plot of the averaged $J(V)$ measurements performed $\pm 1.0 \mathrm{~V}$ on $\mathrm{SAMs}$ of 1-octanedithiol ( $\left.\mathrm{HSC}_{8} \mathrm{SH}\right)$ (2a). 1-dodecanedithiol $\left(\mathrm{HSC}_{12} \mathrm{SH}\right)(\mathbf{2 d}),(5)$ and (6) in PEDOT:PSS/Hg tunneling junctions, inset shows a schematic of the junction structure itself (Figure (b) adapted with permission from. ${ }^{[17 \mathrm{a}]}$ Copyright (C) 2011 American Chemical Society).

\subsubsection{Metal Evaporated Tunneling Junctions}

As briefly mentioned in section 2.2 .3 of this Chapter, metal evaporation is not the most favorable method to create molecular tunneling junctions. The most thorough and critical studies carried out on metal evaporated tunneling junctions were undertaken by Lee and coworkers. $^{\left[24,{ }^{35}\right]} J(V)$ measurements were performed $\pm 1.0 \mathrm{~V}$ on 13440 devices of alkanethiols, ${ }^{[24]}$ 1-octanethiol $\quad\left(\mathrm{C}_{8} \mathrm{SH}\right) \quad(\mathbf{1 b}), \quad$ 1-dodecanethiol $\quad\left(\mathrm{C}_{10} \mathrm{SH}\right) \quad(\mathbf{1 d}), \quad$ and 1-hexadecanethiol $\left(\mathrm{C}_{16} \mathrm{SH}\right)(\mathbf{1 g})$ and 14400 devices of the alkanedithiols, ${ }^{[24,35]}{ }^{1,8-}$ octanedithiol ( $\left.\mathrm{HSC}_{8} \mathrm{SH}\right)$ (2a), 1,9-nonanedithiol ( $\left.\mathrm{HSC}_{9} \mathrm{SH}\right)$ (2b) and 1,10-decanedithiol $\left(\mathrm{HSC}_{10} \mathrm{SH}\right)$ (2c) in metal evaporated tunneling junctions, with the top contact being evaporative $\mathrm{Au}$ (Figure 2.12a). From the 13440 devices of the alkanethiols, the authors reported that 11744 were electrical shorts/short circuits, 392 succumbed to fabrication failure, with an additional 1103 electrical open devices which were also attributed to failures during the fabrication process. With the remaining 201 devices a comprehensive statistical analysis was performed on the $J(V)$ data obtained. This led to an exclusion of an additional 45 devices, which were classed as non-working due to their outlying $J$ values. This left only 
156 devices being classified as 'working devices' giving a yield of only $1.2 \%$. The same type of study and analysis was also carried out on 14400 alkanedithiol devices, with the authors reporting 12340 devices as electrical shorts, 472 succumbing to fabrication failure, 1252 being electrical open devices and after statistical analysis an additional 65 being classified as non-working devices, leaving 271 working devices with a working device yield of only $1.9 \%$. However, due to the large sample size, the 156 and 271 working devices provided sufficient data to investigate the charge transport characteristics of the alkanethiols and alkanedithiols, respectively. The authors found that $J$ obtained was clearly dependent on the molecular length (i.e., the longer the molecule, the larger the distance between electrodes) and the metal-molecular contacts (i.e., monothiol vs. dithiol)(Figure 2.12a), ${ }^{[24,35]}$ and determined the tunneling decay constant $\beta$ to be $0.81 \pm 0.05,0.83 \pm 0.04$ and $0.86 \pm 0.06$ for $\mathrm{C}_{8} \mathrm{SH}(\mathbf{1 b}), \mathrm{C}_{10} \mathrm{SH}(\mathbf{1 d})$, and $\mathrm{C}_{16} \mathrm{SH}(\mathbf{1 g})$, respectively, ${ }^{[24]}$ and $0.55 \pm 0.06$, $0.57 \pm 0.06$ and $0.58 \pm 0.08 \AA^{-1}$ for $\mathrm{HSC}_{8} \mathrm{SH}(\mathbf{2 a}), \mathrm{HSC}_{9} \mathrm{SH}(\mathbf{2 b})$ and $\mathrm{HSC}_{10} \mathrm{SH}(\mathbf{2 c})$, respectively (Figure 2.12c). ${ }^{[35]}$ The higher $J$ values and lower $\beta$ values of the alkanedithiols are due to the two chemisorbed contacts (one with each electrode), whereas the alkanethiols possess only one chemisorbed contact with the other being physisorbed. The physisorbed contact leads to a poor tunneling rate, thus giving lower $J$ values and higher $\beta$ values.

From semi-log plots of tunneling current densities at various voltages as a function of the molecular length of the different alkanethiols (data used from these plots were from representative devices chosen from the positions of the mean values in the statistical analysis), the tunneling current densities show that $J$ decreases exponentially with increasing molecular length (Figure 2.12b). ${ }^{[24]}$ This allowed (along with temperature measurements) the authors to determine that the charge transport is through-bond tunneling.

From these studies, the authors were able to propose a 'multibarrier tunneling model' that generalized the traditional Simmons tunneling model. However, this is beyond the scope of this review, more information can be found in the articles by Lee and co-workers. ${ }^{[35,47]}$ 
a)

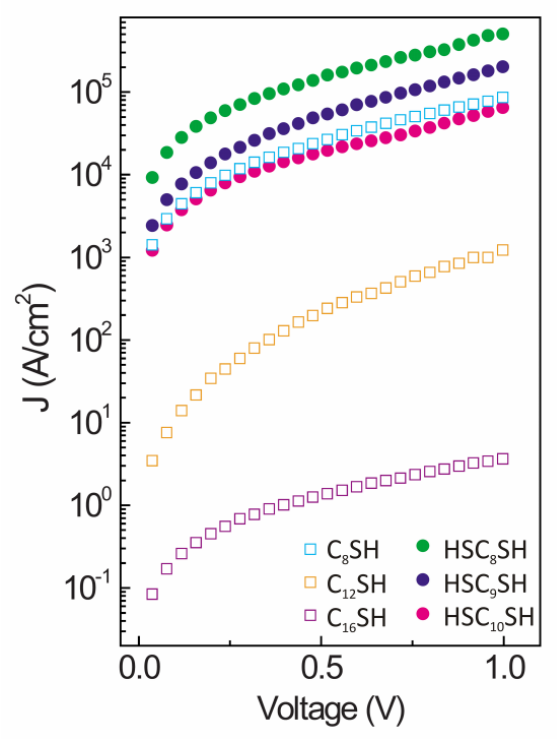

b)

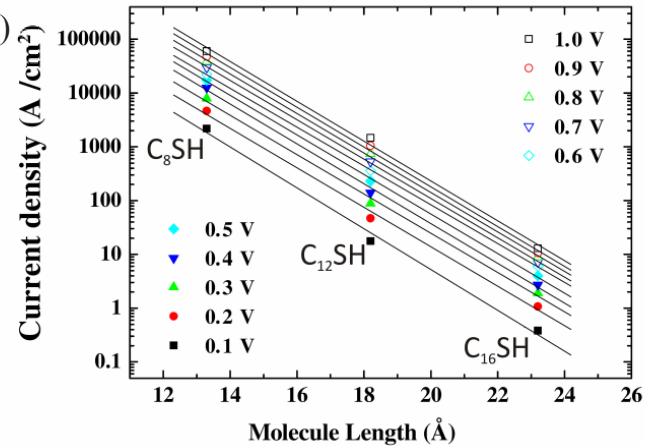

c)

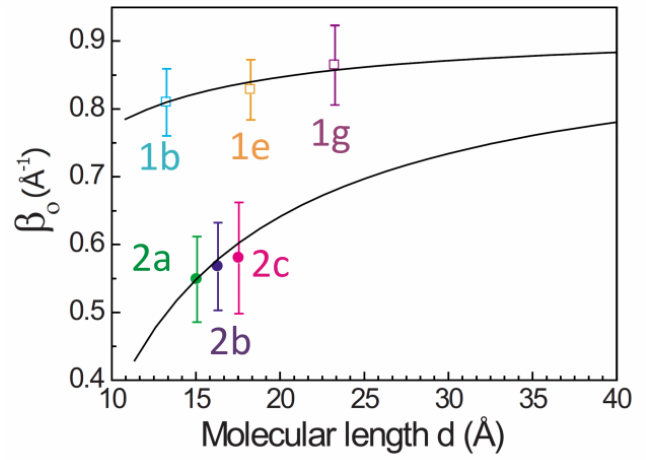

Figure 2.12: (a) Semi-log plot of the $J(V)$ measurements $0 \rightarrow 1.0 \mathrm{~V}$ from representative devices (chosen from the mean positions of the fitted Gaussian functions) for the SAMs $\mathrm{C}_{8} \mathrm{SH}(\mathbf{1 b}), \mathrm{C}_{12} \mathrm{SH}(\mathbf{1 e}), \mathrm{C}_{16} \mathrm{SH}(\mathbf{1 g})$, $\mathrm{HSC}_{8} \mathrm{SH}$ (2a), $\mathrm{HSC}_{9} \mathrm{SH}$ (2b) and $\mathrm{HSC}_{10} \mathrm{SH}$ (2c) in metal evaporated tunneling junctions. (b) Semi-log plot of the averaged values of $J$ measured at different biases in metal evaporated tunneling junctions as a function of the molecular length of the alkanethiols $\mathrm{C}_{8} \mathrm{SH}(\mathbf{1 b}), \mathrm{C}_{12} \mathrm{SH}(\mathbf{1 e}), \mathrm{C}_{16} \mathrm{SH}(\mathbf{1 g})$. (c) Plot of the averaged values of the overall tunneling decay coefficient $\left(\beta_{0}\right)$ vs. molecular length, for SAMs of $\mathrm{C}_{8} \mathrm{SH}(\mathbf{1 b}), \mathrm{C}_{12} \mathrm{SH}(\mathbf{1 e}), \mathrm{C}_{16} \mathrm{SH}$ (1g), $\mathrm{HSC}_{8} \mathrm{SH}(\mathbf{2 a}), \mathrm{HSC}_{9} \mathrm{SH}(\mathbf{2 b})$ and $\mathrm{HSC}_{10} \mathrm{SH}$ (2c) in metal evaporated tunneling junctions. (Figures (a) and (c) reprinted with permission from. ${ }^{[35]}$ Copyright (C) 2007 by the American Physical Society. Figure (b) reprinted with permission from. ${ }^{[24]}$ Copyright (C) 2007 IOPscience). 


\subsection{2 $\pi$ - Conjugated Molecules}

$\pi$ - Conjugated molecules are considered as the prototype of molecular semi-conducting wires (Figure 2.13). ${ }^{[48]}$ These molecular systems have a smaller energy gap between their HOMO and LUMO level than alkane chains, thus for $\pi$ - conjugated molecules longer than $3 \mathrm{~nm}$, hopping is the dominant mechanism of charge transport. ${ }^{[39]}$ However, for $\pi-$ conjugated molecules shorter than $\sim 3 \mathrm{~nm}$, which is typically the length of molecule used in molecular tunneling junctions, the dominant mechanism of charge transport is tunneling. ${ }^{[39]}$ As in the alkanethiols, the tunneling current decays exponentially with the distance between the two electrodes (Equation 2.2), which is proportional to the length of molecule investigated. Published $\beta$ values for $\pi$ - conjugated molecules shorter than $3 \mathrm{~nm}$ in single molecule and large scale self-assembled junctions range from $0.04-0.61 \AA^{-1}{ }^{[49]}$ The structures of the molecules discussed in this section (2.3.2) are given on the next page (page $33)$. 
<smiles>Sc1ccc(C#Cc2ccc3cc(C#Cc4ccc(S)cc4)ccc3c2)cc1</smiles><smiles>Sc1ccc(-c2ccc(S)cc2)cc1</smiles><smiles>Sc1ccc(-c2ccccc2)cc1</smiles>

$10 b$<smiles>O=C1c2ccc(C#Cc3ccc(S)cc3)cc2C(=O)c2ccc(C#Cc3ccc(S)cc3)cc21</smiles>

8<smiles>CC1c2ccc(C#Cc3ccc(S)cc3)cc2Cc2ccc(C#Cc3ccc(S)cc3)cc21</smiles>

9<smiles>Sc1ccc(C#Cc2ccc(-c3ccccc3)cc2)cc1</smiles><smiles>Sc1ccc(-c2cnc(-c3ccccn3)cn2)nc1</smiles>

Figure 2.13: Molecular structure of 4,4'-(anthracene-2,6-diylbis(ethyne-2,1-diyl))dibenzenethiol (7), 2,6bis((4-mercaptophenyl)ethynyl)anthracene-9,10-dione (8) and 4,4'-((9,10-dihydroanthracene-2,6diyl)bis(ethyne-2,1-diyl))dibenzenethiol (9), an oligo-para-phenylene dithiol (10) ([1,1'-biphenyl]-4,4'-dithiol (10b)), an oligo-para-phenylene monothiol (11) ([1,1'-biphenyl]-4-thiol (11b)) an oligophenylene ethynylene dithiol (12) (4,4'-(ethyne-1,2-diyl)dibenzenethiol (12b)), a benzylic derivative of an oligo-para-phenylene thiol (13) ([1,1'-biphenyl]-4-ylmethanethiol (13b)) and 6-(5-pyridin-2-ylpyrazin-2-yl)pyridine-3-thiol (14). 


\subsubsection{EGaIn Tunneling Junction}

To our knowledge, the only $J(V)$ measurements performed on SAMs of conjugated molecules in EGaIn tunneling junctions, were carried out by Chiechi and co-workers on SAMs of three arylethylene thiols/thioates, 4,4'-(anthracene-2,6-diylbis(ethyne-2,1diyl))dibenzenethiol (7), 2,6-bis((4-mercaptophenyl)ethynyl)anthracene-9,10-dione (8) and 4,4'-((9,10-dihydroanthracene-2,6-diyl)bis(ethyne-2,1-diyl))dibenzenethiol (9). ${ }^{[50]}$ The three SAMs are similar in thickness and differ only in their core conjugation patterns, with 7 having a linear conjugated core, 8 having a cross-conjugated core and $\mathbf{9}$ having a broken-conjugated core, all connected at the 2,6 positions (Figure 2.14). After accumulating and analyzing statistically relevant numbers of $J(V)$ data $\pm 0.4 \mathrm{~V}$, at the outermost voltages the linear-conjugated core of 7 appeared at least ten times more conductive than the brokenconjugated and the cross-conjugated cores of $\mathbf{8}$ and $\mathbf{9}$, respectively. Therefore, lower values of $J$ were observed in SAMs where the linear-conjugation was broken. The authors also showed that the experimental data obtained was also in good qualitative agreement with the theoretical transport properties of these three conjugated systems. In this study the yield of working junctions was lower than that of other transport studies carried out on SAMs using the EGaIn technique. ${ }^{[41 b]}$ This is largely because it was not possible to form these SAMs on template stripped metal surfaces, as the adhesive used to bind the metal film to the solid support in the fabrication of the template stripped surfaces, is not compatible with the organic solvents required to dissolve the arylethylene thiols/thioates. Therefore, these SAMs were formed on surfaces of vapor-deposited gold-on-mica, which give large islands of atomically flat $\mathrm{Au}(111)$, but are separated by large step-edges and grain boundaries that function as defects (i.e. the surfaces are 'rough'). As discussed in section 2.2.1, SAMs prepared on these surfaces are far less ordered than those prepared on template stripped surfaces. Thus, the junctions created and measured in this study had only $6 J(V)$ scans performed $\pm 0.4 \mathrm{~V}$, whereas in other EGaIn junction studies where the SAM was formed on template stripped surfaces typically 20 scans were measured $\pm 1.0 \mathrm{~V}$. The rougher surfaces most likely caused a higher percentage of junctions to immediately short (20-30\%). From the junctions that did not short immediately, only $80 \%$ did not short during the $J(V)$ measurements. Typically other EGaIn studies have a total yield of working junctions of $\sim 85 \%$. Therefore, when possible, SAMs should be formed on template stripped surfaces. 


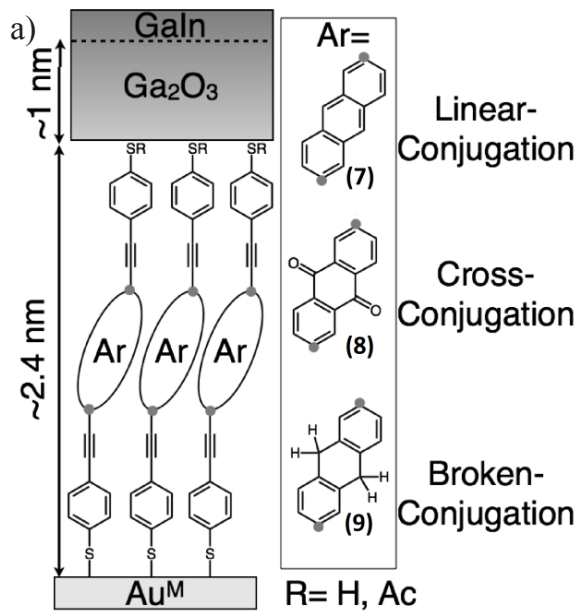

b)

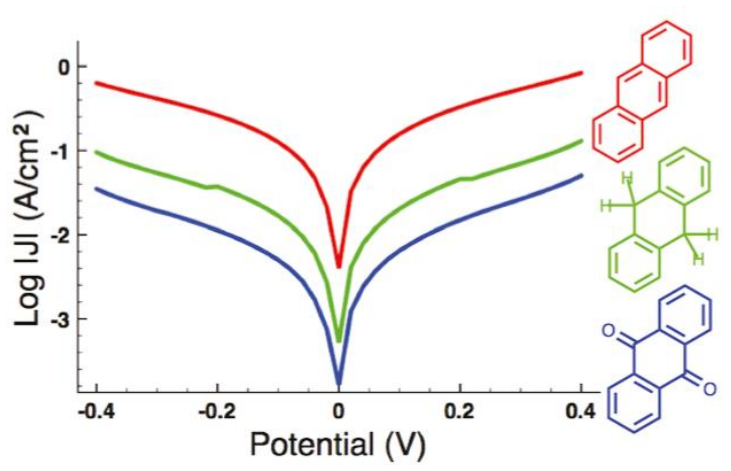

Figure 2.14: (a) Schematic of the EGaIn tunneling junctions, with SAMs of thiolated arylethynylenes, 4,4'(anthracene-2,6-diylbis(ethyne-2,1-diyl))dibenzenethiol (7) having a linear conjugated core, 2,6-bis((4mercaptophenyl)ethynyl)anthracene-9,10-dione (8) having a crossed conjugated core and 4,4'-((9,10dihydroanthracene-2,6-diyl)bis(ethyne-2,1-diyl))dibenzenethiol (9) having a broken conjugated core, all connected at the 2,6 positions (indicated with gray circles) formed on a vapor deposited Au surface. (b) Semi$\log$ plots of the geometric mean of the $J(V)$ data obtained for 7 (red), 8 (blue) and $\mathbf{9}$ (green) in EGaIn tunneling junctions. (Figures (a) and (b) adapted with permission from. ${ }^{[50]}$ Copyright (C) 2011 American Chemical Society)

\subsubsection{PEDOT:PSS Tunneling Junctions}

The $J(V)$ characteristics of a broader range of conjugated molecules have been investigated using the PEDOT:PSS technique. De Leeuw and co-workers performed a systematic lengthdependent study on SAMs of $\pi$ - conjugated oligo-para-phenylene dithiols (10) and monothiols (11), with an increasing number of phenylene rings. ${ }^{[30,49]}$ The molecules investigated were 1,4-benzenedithiol (10a), [1,1'-biphenyl]-4,4'-dithiol (10b), [1,1':4'1'”terphenyl]-4,4"-dithiol (10c), [1,1':4',1":4",1"'-quaterphenyl]-4,4"'-dithiol (10d), 1benzenethiol (11a), [1,1'-biphenyl]-4-thiol (11b) and [1,1':4'1'-terphenyl]-4-thiol (11c), as shown in Table 1 . The $J(V)$ characteristics of these SAMs were determined by performing statistically relevant numbers of scans $\pm 0.5 \mathrm{~V}$. Figure 2.15 displays the normalized resistance $(R S)$ at $0.5 \mathrm{~V}$, as a function of molecular length for the conjugated series 10a-d, 
11a-c, and for junctions containing only PEDOT:PSS. Each data point is an average of $>240$ devices with diameters from $5-50 \mu \mathrm{m}$. The authors found that for both series of molecules the $R S$ increases exponentially with molecular length (i.e. $J$ decreases exponentially with molecular length). They determined the tunneling decay coefficient $(\beta)$ to be $0.26 \pm 0.04 \AA^{-1}$ for $10(10 a-10 d)$ and $0.20 \pm 0.06 \AA^{-1}$ for $11(11 a-11 d)$. The $R S$ values found for the phenylenes $\mathbf{1 0}$ and $\mathbf{1 1}$ in the PEDOT:PSS junctions are higher than that found for the alkanes $\mathbf{1}$ and $\mathbf{2}$ in the same junction technique, which the authors attribute to a smaller fraction of contacted molecules.

The $\beta$ found for the SAMs of phenylenes $\left(0.26 \AA^{-1} \text { for } \mathbf{1 0} \text { and } 0.20 \AA^{-1} \text { for } \mathbf{1 1}\right)^{[30,49]}$ was smaller than $\beta$ found for the SAMs of alkanethiols (1) and alkanedithiols (2) also determined in the PEDOT:PSS tunneling junctions (as discussed earlier, $0.66 \AA^{-1}$ for 1 and $0.73 \AA^{-1}$ for 2). ${ }^{[20,30-31,44]}$ Smaller values of $\beta$ are expected for $\pi$ - conjugated molecules as they have a smaller HOMO - LUMO gap than alkanethiols. Therefore, the $\beta$ values found in the PEDOT:PSS junctions agree with this theory. Interestingly, the authors also reported that the $\beta$ values found for SAMs of phenylenes in PEDOT:PSS tunneling junctions were lower than that reported for single molecules and phenelyne SAMs in a $\mathrm{Hg}$ tunneling junctions (as will be discussed in section 2.3.2.3). By referring to theoretical studies carried out by Zhang and co-workers ${ }^{[51]}$ and Yoshizawa and co-workers, ${ }^{[52]}$ the authors attributed the lower values of $\beta$, in comparison to single molecule measurements, to the para-phenylene system having no torsion angle between adjacent rings when being in a densely packed SAM and thus being in a planar geometry. The maximization of the $\pi-\pi$ interactions of the planar-para-phenylene SAMs, would lower the HOMO-LUMO gap yielding smaller decay coefficients. Therefore, the authors tentatively believe that it can be expected to obtain lower values of $\beta$ for SAMs of $\pi$-conjugated molecules, than single $\pi$-conjugated molecules. The difference in $\beta$ determined for SAMs of phenylenes in PEDOT:PSS tunneling junctions and $\mathrm{Hg}$ drop tunneling junctions, may be due to one or many of the uncertainties associated with these top electrodes. 
Self-Assembled Monolayers in Large Area Molecular Tunneling Junctions

\begin{tabular}{|c|c|c|c|c|}
\hline Molecule & Structure & $\begin{array}{l}\text { Molecular Length } \\
(\AA)\end{array}$ & $\begin{array}{c}\text { Monolayer Thickness } \\
\text { (A) }\end{array}$ & $\begin{array}{l}\text { Tilt Angle } \\
\text { (Deg.) }\end{array}$ \\
\hline $10 \mathrm{a}$ & & 8.9 & 5.8 & 49 \\
\hline $10 \mathrm{~b}$ & & 13.3 & 9.8 & 43 \\
\hline $10 \mathrm{c}$ & & 17.6 & 14.7 & 33 \\
\hline 10d & & 21.8 & 20.6 & 17 \\
\hline $11 \mathrm{a}$ & & 7.9 & 2.4 & 72 \\
\hline $11 b$ & & 12.3 & 9.6 & 38 \\
\hline $11 \mathrm{c}$ & & 16.6 & 15.7 & 17 \\
\hline
\end{tabular}

Table 2.1: Thicknesses of the SAMs of oligo-para-phenylenedithiol (10) and oligo-para-phenylenemonothiol (11) SAMs as determined from ellipsometry measurements (Table adapted with permission from ${ }^{[49]}$ Copyright C 2010 , American Institute of Physics, and from. ${ }^{[30]}$ Copyright $(C)$ 2011, Auke Jisk Kronemeijer).

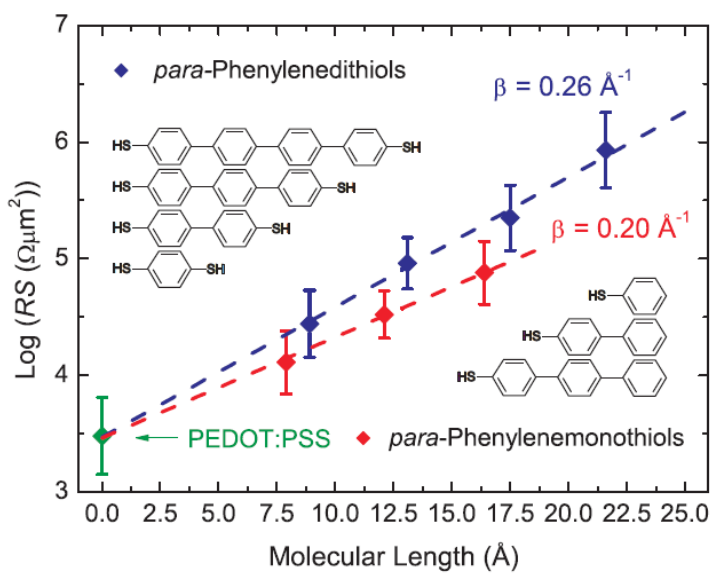

Figure 2.15: Semi-log plot of the normalized resistance $(R S)$ as a function of molecular length for 10a - 10d and 11a - 11c in PEDOT:PSS tunneling junctions. Each data point is an average of at least 240 junctions of 5$50 \mu \mathrm{m}$ in diameter. Error bars represent 1 standard deviation (Figure reprinted with permission from. ${ }^{[49]}$ Copyright $(\subset$ 2010, American Institute of Physics).

The SAMs of three arylethylene thiols, 4,4'-(anthracene-2,6-diylbis(ethyne-2,1diyl))dibenzenethiol (7), 2,6-bis((4-mercaptophenyl)ethynyl)anthracene-9,10-dione (8) and 4,4'-((9,10-dihydroanthracene-2,6-diyl)bis(ethyne-2,1-diyl))dibenzenethiol (9), which have either a linear-conjugated core (7), a cross-conjugated (8) or a broken-conjugated core (9) connected at the 2,6 positions, measured in the EGaIn junctions (as discussed in section 2.3.2.1) were also measured in the PEDOT:PSS junctions. The PEDOT:PSS $J(V)$ measurements displayed no clear difference in the value of $J$ measured for the three 
molecules ${ }^{[53]}$ whereas the EGaIn junctions did show a difference in the value of $J$ measured for the three molecules. This clearly illustrates the experimental differences in $J(V)$ measurements when using different techniques to form the top contact.

Hummelen and co-workers used PEDOT:PSS tunneling junctions as an additional technique along with ellipsometry, X-ray Photon Spectroscopy (XPS) and electrochemistry to characterize the quality of oligophenylene ethynylene (OPE) dithiol (12) SAMs formed on $\mathrm{Au}$ (Figure 2.16b). ${ }^{[54]}$ The SAM was formed via the deprotection of acetyl protected oligophenylene ethynylene dithiols in solution using two different bases, $5-10 \%$ of triethylamine $\left(\mathrm{Et}_{3} \mathrm{~N}\right)$ in THF (which when analyzed via other techniques was found to form dense high quality SAMs), and tetrabutylammonium hydroxide $\left(\mathrm{Bu}_{4} \mathrm{NOH}\right)$ (which when analyzed via other techniques was found to form less dense SAMs along with the incorporation of $\mathrm{Bu}_{4} \mathrm{~N}^{+}$ions into the monolayer). OPE (12) SAMs were also formed in the absence of base, to serve as a control.

A length dependent study was carried out by performing $J(V)$ measurements $\pm 0.5 \mathrm{~V}$ on high quality SAMs of OPE's (12) (formed using 5-10\% $\mathrm{Et}_{3} \mathrm{~N}$ in THF) of different lengths; 1,4benzenedithiol $\left(\mathrm{OPE}_{0}\right)(\mathbf{1 0 a}) /(\mathbf{1 2 a}), 4,4$ '-(ethyne-1,2-diyl)dibenzenethiol $\left(\mathrm{OPE}_{1}\right)(\mathbf{1 2 b}), 4,4$ '(1,4-phenylenebis(ethyne-2,1-diyl))dibenzenethiol $\left(\mathrm{OPE}_{2}\right) \quad(\mathbf{1 2 c})$ and 4,4'-((ethyne-1,2diylbis(4,1-phenylene))bis(ethyne-2,1-diyl))dibenzenethiol $\left(\mathrm{OPE}_{3}\right) \quad(\mathbf{1 2 d}){ }^{[54]}$ For $\mathrm{OPE}_{0-2}$ (12a-c) an exponential decrease in $J$ was found for increasing chain length, with $\beta$ being $0.15 \AA^{-1}$ (Figure 2.16a). For $\mathrm{OPE}_{3}(\mathbf{1 2 d})$ the values of $J$ found were very similar to $J$ found for $\mathrm{OPE}_{2}(\mathbf{1 2 c})$. The authors attributed this observation to a change in the mechanism of charge transport, as the increasing length of the conjugated molecule (as mentioned in section 2.3.2) results in tunneling, which was found for $\mathrm{OPE}_{2}$ (12c) to change to hopping for $\mathrm{OPE}_{3}(\mathbf{1 2 d})$.

To investigate the quality of the SAM formed when using different methods, $J(V)$ measurements were carried out on $\mathrm{OPE}_{2}$ (12c) SAMs formed (as mentioned above) with $10 \% \mathrm{Et}_{3} \mathrm{~N}\left(0.5 \mathrm{mM}\right.$ in THF immersed for two days), with four eq. $\mathrm{Bu}_{4} \mathrm{NOH}(0.3 \mathrm{mM})$ in THF, immersed for one hour) and in the absence of base (0.5 mM in THF for two days). ${ }^{[54]}$ The SAMs formed in the presence of $\mathrm{Et}_{3} \mathrm{~N}$ gave values of $J$ similar to (within the experimental error) the values of $J$ measured for $\mathrm{OPE}_{2}(\mathbf{1 2 c})$ in the length dependent study. SAMs formed in the absence of base gave values of $J$ a factor of ten higher, which was 
attributed to less densely packed SAMs. Interestingly, SAMs formed in the presence of $\mathrm{Bu}_{4} \mathrm{NOH}$, gave values of $J$ two orders of magnitude lower than that found for $\mathrm{OPE}_{2}(\mathbf{1 2 c})$ formed in the presence of $\mathrm{Et}_{3} \mathrm{~N}$. The authors attributed this to either the formation of multilayers, the presence of $\mathrm{Bu}_{4} \mathrm{~N}^{+}$or the adsorption of polymerized disulfides in top of the SAM, therefore, these values of $J$ also indicated the formation of a poor SAM. Thus the $J(V)$ data obtained were in agreement with the data obtained using other experimental techniques, emphasizing that the quality of the SAM formed via the deprotection of acetyl protected oligophenylene ethynylene dithiols is highly dependent on the base used.

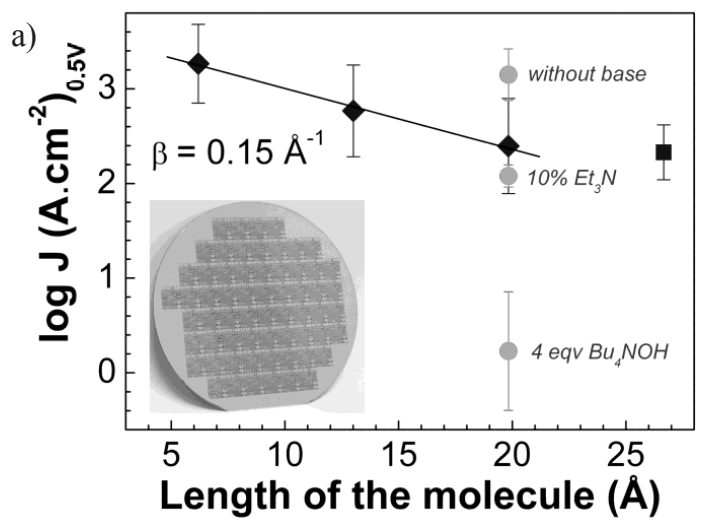

b)

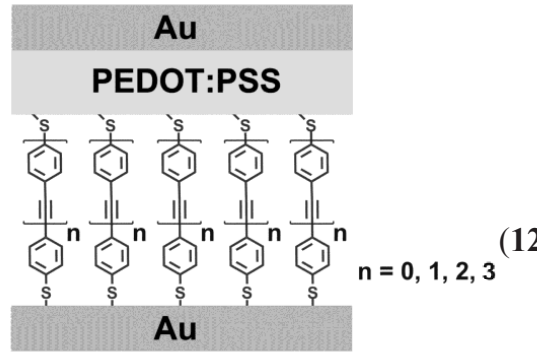

Figure 2.16: (a) Semi-log plot of $J$ as a function of molecular length. Black diamonds represent the average values of $J$ for SAMs of 1,4-benzenedithiol $\left(\mathrm{OPE}_{0}\right)(\mathbf{1 0 a}) /(\mathbf{1 2 a}), 4,4$ '-(ethyne-1,2-diyl)dibenzenethiol $\left(\mathrm{OPE}_{1}\right)$ (12b), 4,4'-(1,4-phenylenebis(ethyne-2,1-diyl))dibenzenethiol $\left(\mathrm{OPE}_{2}\right)$ (12c) and 4,4'-((ethyne-1,2-diylbis(4,1phenylene))bis(ethyne-2,1-diyl))dibenzenethiol $\left(\mathrm{OPE}_{3}\right)(\mathbf{1 2 d})$. Linear fit of $\left(\mathrm{OPE}_{0}\right)(\mathbf{1 0 a}) /(\mathbf{1 2 a})-\left(\mathrm{OPE}_{2}\right)(\mathbf{1 2 c})$ gives $\beta$ of $0.15 \AA^{-1}$. Gray circles represent the average values of $J$ for $\mathrm{SAMs}$ of $\mathrm{OPE}_{2}(\mathbf{1 2 c})$ formed under different conditions. (a, inset) 6 inch wafer with PEDOT:PSS tunneling junctions. (b) Idealized schematic of OPE (12) SAMs immobilized within PEDOT:PSS tunneling junctions. (Figures (a) and (b) reprinted with permission from. ${ }^{[54 \mathrm{~b}]}$ Copyright (C) 2011, E.H. Valkenier-van Dijk.

\subsubsection{Mercury Drop Tunneling Junctions}

In the same study reported above in section 2.3.1.4, Whitesides and co-workers investigated the $J(V)$ characteristics of conjugated SAMs in mercury drop tunneling junctions. ${ }^{[22 \mathrm{~d}, 45]} J(V)$ scans were performed from 0 to $1 \mathrm{~V}$ across oligo-para-phenylene thiol (11) SAMs of 1benzenethiol (11a), [1,1'-biphenyl]-4-thiol (11b) and [1,1':4'1'"-terphenyl]-4-thiol (11c) and the benzylic derivatives of oligo-para-phenylene thiol (13) SAMs of phenylmethanethiol 
(13a), [1,1'-biphenyl]-4-ylmethanethiol (13b) and [1,1':4'1"-terphenyl]-4-ylmethanethiol (13c) (Table 2 and Figure 2.17). The authors reported $\beta$ to be $0.61 \pm 0.1 \AA^{-1}$ and $0.67 \pm 0.1 \AA^{-1}$ at an applied potential of $0.5 \mathrm{~V}$ for $\mathbf{1 1}$ on $\mathrm{Ag}$ and $\mathbf{1 3}$ on $\mathrm{Ag}$, respectively. ${ }^{[45]}$ Thus $J$ measured for SAMs of the same thickness was larger for 11 than 13, with both conjugated SAMs giving larger $J$ values than that of the alkanethiols (1) of the same thickness that were discussed earlier. As in section 2.3.1.4, the same breakdown voltage (BDV) study was carried out with the conjugated molecules. The authors found that the longer the conjugated molecule (i.e. the thicker the SAM) the higher the BDV, and that conjugated (11 and 13) and alkanethiol (1) SAMs of the same thickness, gave similar BDVs. ${ }^{[22 \mathrm{~d}]}$

\begin{tabular}{|c|c|c|c|c|c|}
\hline $\begin{array}{l}\text { Thiol used to form } \\
\text { SAM on Ag }\end{array}$ & $\begin{array}{l}\text { Thiol used to form } \\
\text { SAM on } \mathrm{Hg}\end{array}$ & $\begin{array}{l}\mathbf{d}_{\mathbf{A g}, \mathbf{H}_{\mathbf{g}}} \\
(\mathbf{n m})^{\mathrm{a}}\end{array}$ & $\begin{array}{l}\text { Current Density } \\
\left(\mathbf{A} / \mathrm{cm}^{2}\right)^{\mathbf{b}}\end{array}$ & $\begin{array}{c}\text { BDV } \\
(\mathbf{V})\end{array}$ & $\begin{array}{c}\text { BD-Field } \\
\text { (GV/m) }\end{array}$ \\
\hline (11a)HS- $-\square$ & $\mathrm{HS}\left(\mathrm{CH}_{2}\right)_{15} \mathrm{CH}_{3}$ & $3.2 \pm 0.2$ & $7 \times 10^{-4}$ & $0.7 \pm 0.2$ & $0.22 \pm 0.08$ \\
\hline (13a) $\mathrm{HSCl}$ & & $3.3 \pm 0.2$ & $2 \times 10^{-4}$ & $0.7 \pm 0.1$ & $0.21 \pm 0.05$ \\
\hline (11b) HS- & & $3.7 \pm 0.2$ & $8 \times 10^{-5}$ & $1.4 \pm 0.4$ & $0.4 \pm 0.1$ \\
\hline$(13 b) \mathrm{HS}$ & & $3.7 \pm 0.2$ & $6 \times 10^{-5}$ & $1.5 \pm 0.1$ & $0.41 \pm 0.05$ \\
\hline 1 & & $4.1 \pm 0.2$ & $3 \times 10^{-6}$ & $2.0 \pm 0.2$ & $0.49 \pm 0.08$ \\
\hline (13c) HS & & $4.2 \pm 0.2$ & $5 \times 10^{-7}$ & $1.7 \pm 0.1$ & $0.40 \pm 0.05$ \\
\hline
\end{tabular}

Table 2.2: Summary of data obtained by Whitesides et al in $\pi$-conjugated $\mathrm{Hg}$ tunneling junctions; Distance between $\mathrm{Ag}$ and $\mathrm{Hg}$ electrodes $(\mathrm{dAg}, \mathrm{Hg})$, Current Density $(J)$ measured at $0.5 \mathrm{~V}$, Breakdown Voltage (BDV) and Breakdown Field (BD-Field). (Table adapted with permission from. ${ }^{[45 a]}$ Copyright (C) 2001, American Chemical Society.) 


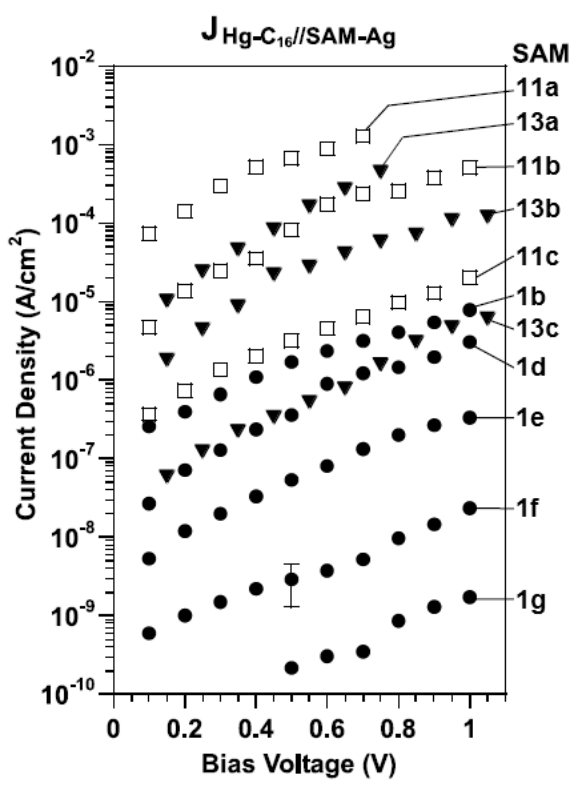

Figure 2.17: Semi-log plot of the averaged $J(V)$ data measured $0 \rightarrow 1.0 \mathrm{~V}$ for SAMs of $\mathrm{C}_{8} \mathrm{SH}(\mathbf{1 b}), \mathrm{C}_{10} \mathrm{SH}$ (1d), $\mathrm{C}_{12} \mathrm{SH}$ (1e), $\mathrm{C}_{14} \mathrm{SH}$ (1f), $\mathrm{C}_{16} \mathrm{SH}$ (1g), 1-benzenethiol (11a), [1,1'-biphenyl]-4-thiol (11b) and [1,1':4'1”terphenyl]-4-thiol (11c), phenylmethanethiol (13a), [1,1'-biphenyl]-4-ylmethanethiol (13b) and [1,1':4'1'”terphenyl]-4-ylmethanethiol (13c) formed on $\mathrm{Ag}$ vapor deposited surfaces in $\mathrm{Hg} / \mathrm{C} 16$ tunneling junctions (Figure adapted from, ${ }^{[23]}$ Copyright (C) 2002 with permission from Elsevier).

Zharnikov and co-workers investigated the influence of nitrogen atoms in the backbone of conjugated SAMs on the $J(V)$ characteristics. ${ }^{[55]}$ The authors performed statistically relevant numbers of $J(V)$ measurements across SAMs of 6-(5-pyridin-2-ylpyrazin-2-yl)pyridine-3thiol (14) and $\left[1,1^{\prime}: 4\right.$ ' 1'"-terphenyl]-4-thiol (11c) on Au and Ag, in mercury drop tunneling junctions (Figure 2.18). They found that throughout the bias regime, the value of $J$ measured for $\mathbf{1 4}$ was more than one order of magnitude lower than that measured for 11c. As $\mathbf{1 4}$ forms high quality SAMs, the authors attributed the lower values of $J$ to the electronic effect of the nitrogen atoms weakening the thiol-metal bond and additionally causing a distortion in the intermolecular interactions, leading to a lower packing density (by 20\%) than that found for 11c. Additionally, as a control $J(V)$ measurements were performed on SAMs of dodecanethiol $\left(\mathrm{C}_{12} \mathrm{SH}\right)(\mathbf{1 e})$. Lower values of $J$ were found for $\left(\mathrm{C}_{12} \mathrm{SH}\right)(\mathbf{1 e})$ than for the 
$\pi$-conjugated molecules of 11c and 14, which is consistent with sets of measurements performed in similar studies.

a)<smiles>Sc1ccc(-c2cnc(-c3ccccn3)cn2)nc1</smiles>

14<smiles>Sc1ccc(-c2ccc(-c3ccccc3)cc2)cc1</smiles>

$11 \mathrm{c}$ b)

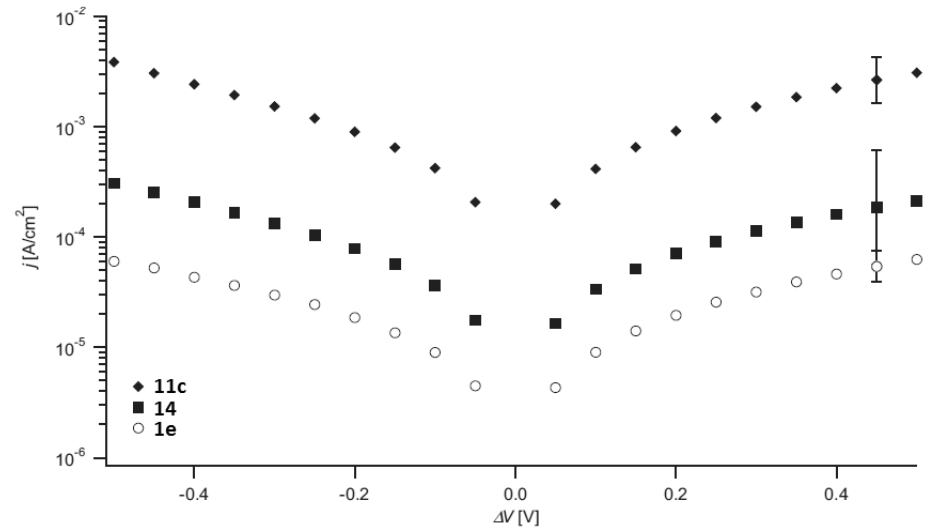

Figure 2.18: (a) Molecular structure of 6-(5-pyridin-2-ylpyrazin-2-yl)pyridine-3-thiol (14) and [1,1':4'1'terphenyl]-4-thiol (11c). (b) Semi-log plot of the averaged $|J|(V)$ data measured $\pm 0.5 \mathrm{~V}$ for SAMS of (14), (11c) and dodecanethiol $\left(\mathrm{C}_{12} \mathrm{SH}\right)(\mathbf{1 e})$ in $\mathrm{Hg} / \mathrm{C}_{12} \mathrm{SH}$ tunneling junctions. (Figure (b) adapted with permission from. ${ }^{[55]}$ Copyright $(\mathcal{C}$ 2007, John Wiley and Sons.)

\subsubsection{Hybrid Tunneling Junctions}

In the same study discussed in section 2.3.1.5, Rampi and co-workers investigated the $J(V)$ characteristics of the $\pi$-conjugated SAM of [1,1':4' 1"-terphenyl]-4-thiol (11a) in PmPV/Hg drop tunneling junctions. ${ }^{[25]}$ The values of $J$ measured were one order of magnitude higher than that of the alkanethiol SAM of similar thickness, 1-dodecanethiol $\left(\mathrm{C}_{12} \mathrm{SH}\right)(\mathbf{1 e})$. This trend is consistent with similar types of $J(V)$ studies in other molecular tunneling junction techniques. From measuring this single SAM the authors estimated $\beta$ to be $0.61 \pm 0.03 \AA^{-1}$ for oligo-para-phenylenes (11) in PmPV/Hg tunneling junctions. 


\subsubsection{Ferrocene Alkanethiol}

The groups of Williams and co-workers, ${ }^{[38 c]}$ and Baranger and co-workers, ${ }^{[38 b]}$ proposed that molecules like ferrocene alkanethiols, possess the appropriate molecular characteristics to function as molecular diodes and thus rectify currents. These authors proposed that in order for the junction to rectify currents, the junction must contain a single conducting molecular orbital such as a HOMO or LUMO, that is slightly offset in energy from the Fermi levels of the electrodes, and asymmetrically situated within the junction, coupled with one of the electrodes (i.e., positioned in closer spatial proximity to one electrode than the other). To fulfill these requirements the molecules immobilized within the molecular tunneling junction must contain three attributes, all of which the ferrocene alkanethiols (15) possess: First, an active headgroup to bind the molecule to the electrode(s) (i.e. a thiol group, Figure $2.19,(a))$, second, a conductive chemical functional group that possesses an energetically accessible HOMO or LUMO level (a ferrocene moiety contains an energetically accessible HOMO level, Figure 2.19, (c)), and third and finally, an insulating component to provide molecular asymmetry within the junction (the alkane chain can provide the appropriate asymmetry, Figure 2.19, (b)). Therefore, theoretically ferrocene alkanethiols should function as the perfect molecular diode, and be the origin of rectification in molecular tunneling junctions. The molecular structures of the different ferrocene alkanethiols discussed in this section are shown on the next page in Figure 2.19. 


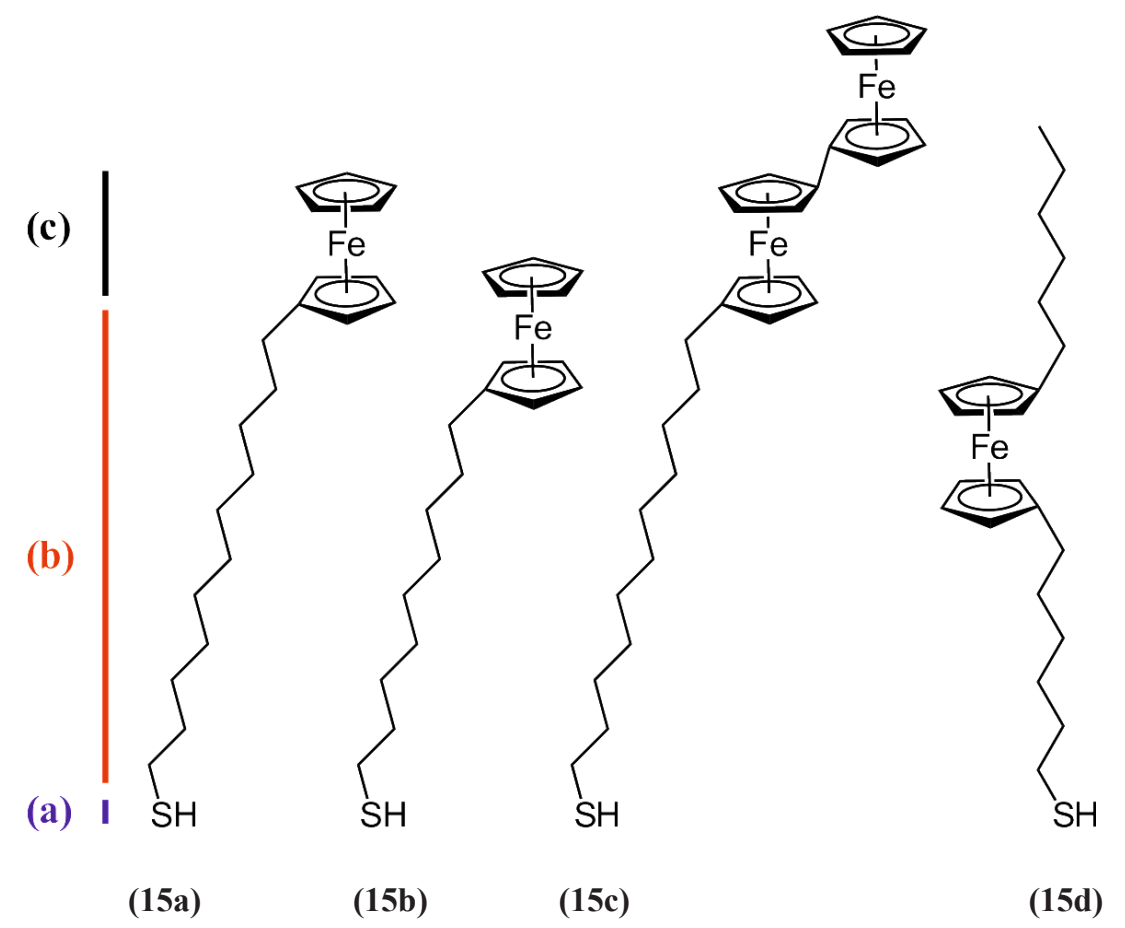

Figure 2.19: Molecular structures of the ferrocene alkanethiols: $\mathrm{SC}_{11} \mathrm{Fc}(15 \mathbf{a}), \mathrm{SC}_{9} \mathrm{Fc}(\mathbf{1 5 b}), \mathrm{SC}_{11} \mathrm{Fc}_{2}(\mathbf{1 5 c})$, $\mathrm{SC}_{6} \mathrm{FcC}_{5} \mathrm{CH}_{3}$ (15d). In $\mathrm{SC}_{11} \mathrm{Fc}$ (15a), (a) indicates the thiol headgroup, (b) indicates the alkane chain which is the insulating component and (c) indicates a ferrocene terminal functional group which contains an energetically accessible HOMO level.

\subsubsection{EGaIn Tunneling Junctions}

Whitesides and co-workers carried out extensive charge transport studies on SAMs of ferrocene alkanethiols in EGaIn tunneling junctions (Figure 2.20a). ${ }^{[27,41]}$ From statistically relevant numbers of $J(V)$ measurements $\pm 1.0 \mathrm{~V}$, the SAMs of ferrocene alkanethiols, $\mathrm{SC}_{11} \mathrm{Fc}$ (15a), on $\mathrm{Ag}^{\mathrm{TS}}$, were found to rectify currents at a negative bias, with a rectification ratio $(R)$ of $1.0 \times 10^{2}(\mathrm{R}=|J(-\mathrm{V})||J(+\mathrm{V})|$ at $\pm 1.0 \mathrm{~V}$ with a $\log$ standard deviation of 3.0) (Figure $2.20 \mathrm{~b}){ }^{[27]}$ Within this tunneling junction, the ferrocene $(\mathrm{Fc})$ moiety forms a van der Waals contact with the $\left(\mathrm{Ga}_{2} \mathrm{O}_{3}\right)$ EGaIn top electrode and is separated from the $\mathrm{Ag}^{\mathrm{TS}}$ bottom electrode by the aliphatic carbon chain. The HOMO level of the Fc moiety is therefore 
asymmetrically placed close to and coupled with the orbitals of the $\left(\mathrm{Ga}_{2} \mathrm{O}_{3}\right)$ EGaIn topelectrode. The estimated energy for the HOMO level of the Fc moiety (from cyclic voltammetry measurements) was $-5.0 \mathrm{eV}$, which lies very close to the Fermi levels of the electrodes, $\sim-4.7 \mathrm{eV}$ for $\mathrm{Ag}$ and $-4.3 \mathrm{eV}$ for $\left(\mathrm{Ga}_{2} \mathrm{O}_{3}\right)$ EGaIn. As when performing the $J(V)$ measurements the authors biased the $\left(\mathrm{Ga}_{2} \mathrm{O}_{3}\right)$ EGaIn top electrode and connected the $\mathrm{Ag}^{\mathrm{TS}}$ bottom electrode to ground, they originally deduced the following hypothesis for the mechanism of charge transport.

a)

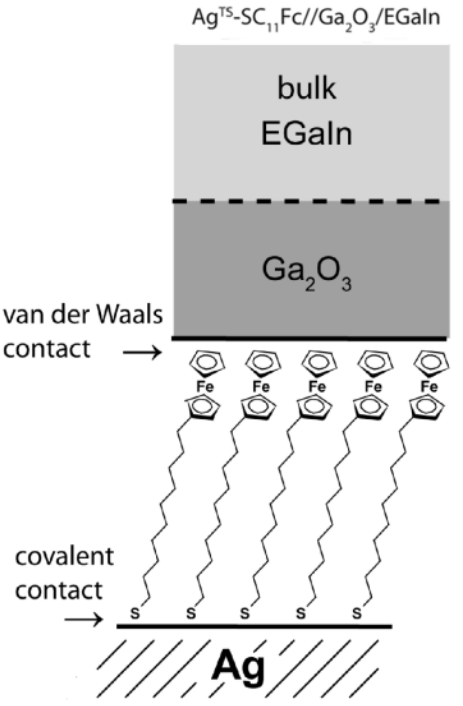

b)

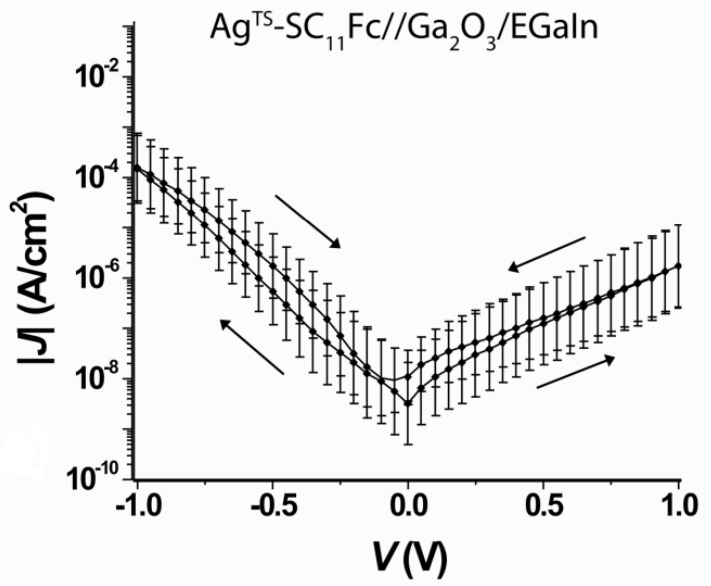

Figure 2.20: (a) Idealized schematic of the ferrocene alkanethiol SAM $\left(\mathrm{SC}_{11} \mathrm{Fc}\right)(\mathbf{1 5 a})$ immobilized on $\mathrm{Ag}^{\mathrm{TS}}$ within an EGaIn tunneling junction. (b) Semi-log of the averaged $|J|(V)$ measurements performed $\pm 1.0 \mathrm{~V}$, for $\mathrm{SAMs}$ of $\mathrm{SC}_{11} \mathrm{Fc}$ (15a) in EGaIn tunneling junctions (Figures (a) and (b) reprinted with permission from. ${ }^{[27]}$ Copyright (C) 2009, American Chemical Society).

The authors hypothesized that during a $J(V)$ scan $\pm 1.0 \mathrm{~V}$, most of the potential drops across the aliphatic carbon chain causing the Fe moiety to follow the potential of the $\left(\mathrm{Ga}_{2} \mathrm{O}_{3}\right) \mathrm{EGaIn}$ top-electrode. At a positive bias, the Fermi level of the $\left(\mathrm{Ga}_{2} \mathrm{O}_{3}\right)$ EGaIn top-electrode would decrease (from $-4.3 \mathrm{eV}$ to $-5.3 \mathrm{eV}$ ) and likewise the HOMO level of the Fc moiety (from $-5.0 \mathrm{eV}$ to $-5.7 \mathrm{eV}$ ) (Figure 2.21). The HOMO level of the Fc moiety would not change to the same degree as the Fermi level of the $\left(\mathrm{Ga}_{2} \mathrm{O}_{3}\right)$ EGaIn top electrode, which is thought to be due to the applied potential dropping across the van der Waals interface. In 
this case the Fc moiety would not be able to participate in charge transport, as its HOMO level would not overlap with the Fermi levels of both electrodes, therefore suggesting that tunneling is the dominant mechanism of charge transport at a positive bias. At a negative bias, the Fermi level of the $\left(\mathrm{Ga}_{2} \mathrm{O}_{3}\right) \mathrm{EGaIn}$ top-electrode would increase (from $-4.3 \mathrm{eV}$ to $-3.3 \mathrm{eV}$ ) and likewise the HOMO level of the $\mathrm{Fc}$ (from $-5.0 \mathrm{eV}$ to $-4.3 \mathrm{eV}$ ). In this case, the Fc moiety would be able to participate in charge transport, as its HOMO level does overlap with the Fermi levels of both electrodes. Therefore, this suggested that hopping is a possible mechanism of charge transport at a negative bias. Thus, the larger values of $J$ measured at a negative bias compared to the values of $J$ measured at a positive bias, which clearly display rectification, are due to the Fc moiety only being able to participate in charge transport at a negative bias.

positive bias

$$
V=1.0 \mathrm{~V}
$$

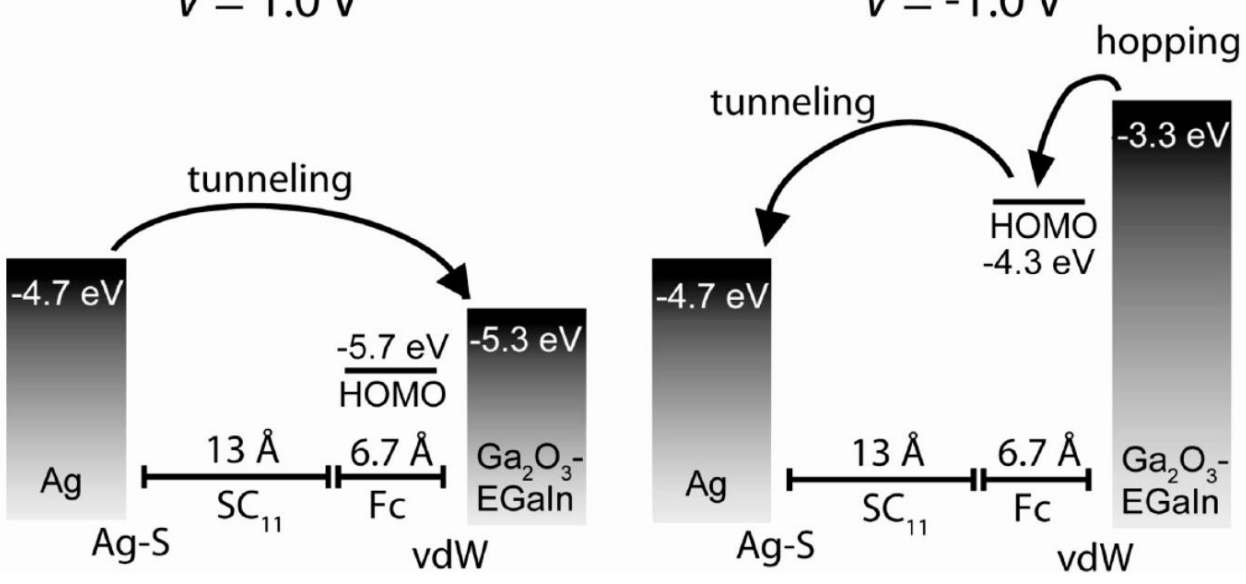

\section{negative bias}

$V=-1.0 \mathrm{~V}$

hopping

Figure 2.21: The proposed energy level diagram for the mechanism of charge transport across the ferrocene alkanethiol SAM $\left(\mathrm{SC}_{11} \mathrm{Fc}\right) \mathbf{( 1 5 a )}$ immobilized on $\mathrm{Ag}^{\mathrm{TS}}$ in EGaIn tunneling junctions. This allows the charge to hop from the $\left(\mathrm{Ga}_{2} \mathrm{O}_{3}\right)$ EGaIn top electrode to the Fc moiety, thus reducing the width of the tunneling barrier (Figure reprinted with permission from. ${ }^{[41 \mathrm{~b}]}$ Copyright (C) 2010, American Chemical Society).

To experimentally investigate the hypothesis that the dominant mechanism of charge transport is electron hopping at a negative bias and electron tunneling at a positive bias, thus, causing rectification to occur, Whitesides and co-workers, performed temperature dependent $J(V)$ measurements $\pm 1.0 \mathrm{~V}$, over a temperature range of 110 to $293 \mathrm{~K}$, across small arrays of tunneling junctions consisting of $\mathrm{SC}_{11} \mathrm{Fc}(\mathbf{1 5 a})$ immobilized on $\mathrm{Ag}^{\mathrm{TS}}$ bottom electrodes, with 46 
the $\left(\mathrm{Ga}_{2} \mathrm{O}_{3}\right)$ EGaIn top electrodes stabilized in microchannels of the polymer polydimethylsiloxane (PDMS) (Figure 2.22). ${ }^{[41 \mathrm{~b}]}$ In this study the values of $J$ obtained depended on temperature at a negative bias but were (nearly) independent of temperature at a positive bias (Figure 2.22). This confirmed that tunneling (which is temperature independent) dominated the mechanism of charge transport at a positive bias and that hopping (which is temperature dependent) dominated the mechanism of charge transport at a negative bias.
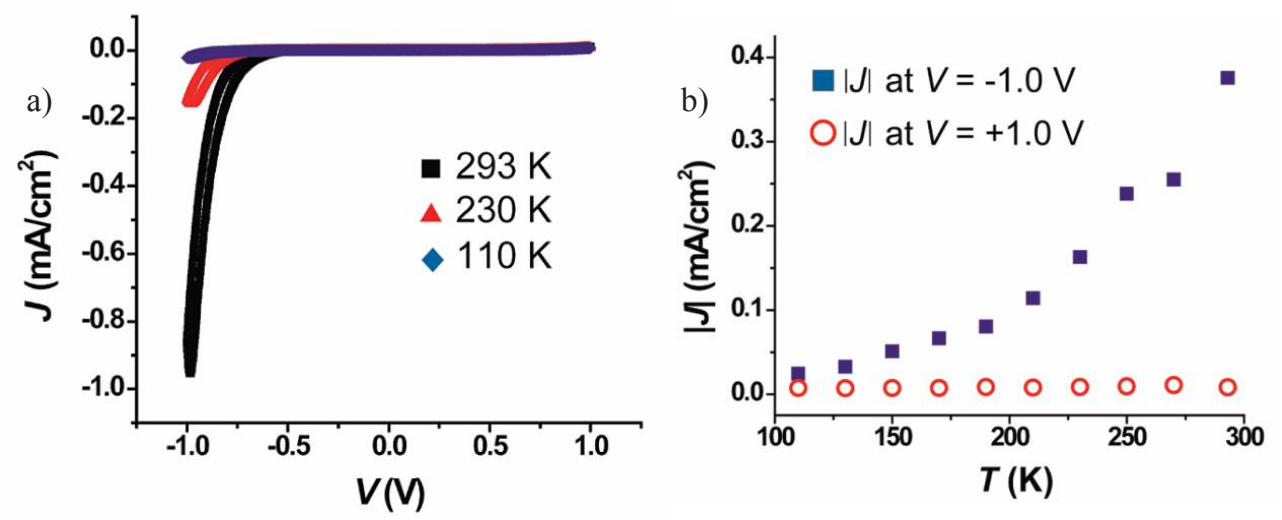

Figure 2.22: (a) Three $J(V)$ measurements performed at three different temperatures (110, 230 and $293 \mathrm{~K})$ and (b) values of $J$ measured at $-1.0 \mathrm{~V}$ and $+1.0 \mathrm{~V}$ as a function of temperature, for $\mathrm{SAMs}_{\mathrm{S}} \mathrm{SC}_{11} \mathrm{Fc}(\mathbf{1 5 a})$ in microchannel EGaIn tunneling junctions. (Figures (a) and (b) reprinted with permission from. ${ }^{[41 b]}$ Copyright $(\mathrm{C}$ 2010, American Chemical Society.)

In an extended study, Whitesides and co-workers further manipulated the ability of ferrocene alkanethiol molecules to rectify current in EGaIn tunneling junctions by investigating the $J(V)$ characteristics of an additional three ferrocene alkanethiol molecules. ${ }^{[41 \mathrm{a}]}$ Relative to the ferrocene alkanethiol previously investigated, $\mathrm{SC}_{11} \mathrm{Fc}(\mathbf{1 5 a})$, which gave a $R$ of $1.0 \times 10^{2}$ (with a $\log$ standard deviation of 3.0), the additional ferrocene alkanethiols either possess a shorter insulating component, $\mathrm{SC}_{9} \mathrm{Fc}(\mathbf{1 5 b})$, giving an $R$ of 10 (with a $\log$ standard deviation of 6.8), a longer conductive component, $\mathrm{SC}_{11} \mathrm{Fc}_{2}$ (15c), giving an $R$ of $5.0 \times 10^{2}$ (with a $\log$ standard deviation of 3.5) or have the HOMO level placed symmetrically within the tunneling junction (rather than asymmetrically), $\mathrm{SC}_{6} \mathrm{FcC}_{5} \mathrm{CH}_{3}$ (15d), giving an $R$ of 1.2 (with a log standard deviation of 1.7). The authors found that these 
experimental data agreed with the theoretical model of rectification proposed by Baranger et al., ${ }^{[38 b]}$ but disagreed with the models proposed by Williams et al., ${ }^{[38 c]}$ and Ford et al. ${ }^{[38 d]}$

Recently, Whitesides and co-workers were also able to perform alternating current (AC) measurements on SAMs of the ferrocene alkanethiols of $\mathrm{SC}_{11} \mathrm{Fc}$ (15a) and $\mathrm{SC}_{11} \mathrm{Fc}_{2}(\mathbf{1 5 c})$ immobilized within EGaIn tunneling junctions (Figure 2.23). ${ }^{[5]}$ The authors found that the $\mathrm{AC}$ circuits operating at $50 \mathrm{~Hz}$ were stable over a larger bias range than the direct current (DC) circuits, allowing for an investigation of charge transport over biases unattainable in DC measurements. They determined that at a reverse bias, two different regimes of charge transport were present. At $|V|<2.0 \mathrm{~V}$, direct tunneling dominated the mechanism of charge transport, with Fowler - Nordheim $(\mathrm{FN})$ tunneling ${ }^{[32]}$ then dominating charge transport at $|V| \approx 2.0 \mathrm{~V}$. However, at a forward bias three different regimes of charge transport were present; direct tunneling $(V=0-0.5 \mathrm{~V})$, hopping plus direct tunneling $(V \approx 0.5-2.0 \mathrm{~V})$ and FN tunneling $(\mathrm{V}>\sim 2.0 \mathrm{~V})$. Due to the difference in mechanisms of charge transport at forward and reverse biases, large rectification values were obtained $(R>100)$, allowing half wave rectification.

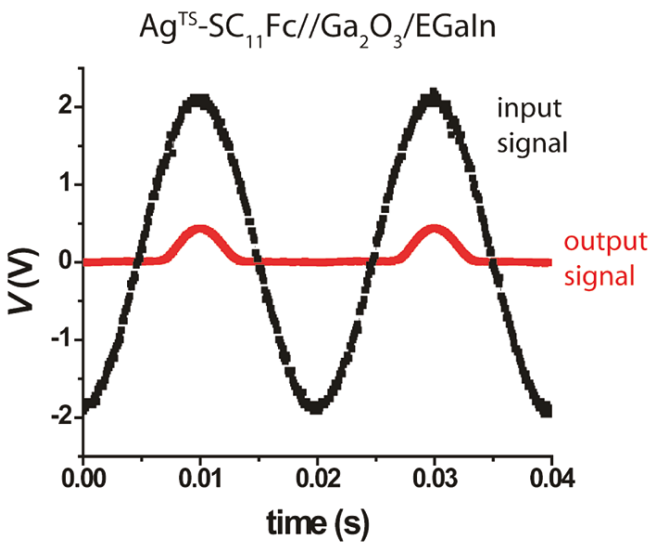

Figure 2.23: Plot of the sinusoidal input signals $\left(\mathrm{V}_{\mathrm{in}}\right.$, black $)$ with corresponding output signal $\left(\mathrm{V}_{\text {out }}\right.$, red $)$ as a function of time, for alternating current (AC) measurements performed across $\mathrm{SAMs}$ of $\mathrm{SC}_{11} \mathrm{Fc}$ (15a) in EGaIn tunneling junctions. Input signal is sinusoidal $(50 \mathrm{~Hz}$ ), with a peak voltage of $2.1 \mathrm{~V}$ (Figure reprinted with permission from. ${ }^{[56]}$ Copyright $(C)$ 2011, American Chemical Society). 


\subsubsection{Organometallic SAMs}

Organometallic SAMs are interesting for the use in molecular tunneling junctions due to their reversible electrochemical and photochemical properties, which can be tuned by varying either the ancillary ligands present on the metal centers or changing the oxidation states of the metal. ${ }^{[57]}$ The problem with these SAMs is that they are complicated molecular systems for molecular tunneling junction standards, and therefore, it is difficult to deduce their true charge transport mechanism. Discussed in this section (2.3.4) are studies that have attempted to obtain a greater understanding of the charge transport mechanism across organometallic SAMs. The molecular structures of the different organometallic systems discussed in this section (2.3.4) are in Figure 2.24, on the next page (page 50). Please note that the molecular structure for molecules $\mathbf{2 1} \mathbf{- 2 4}$ is not given, as no experimental $J(V)$ data is shown for these molecules. 


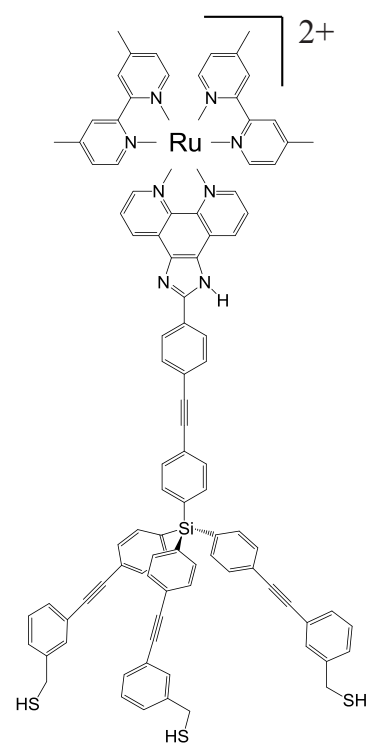

(16)

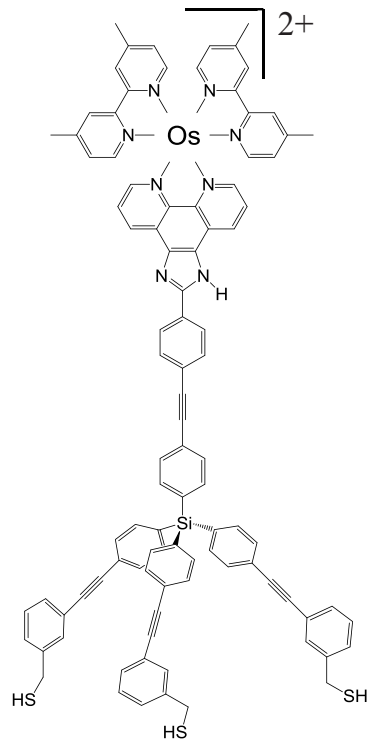

(17)

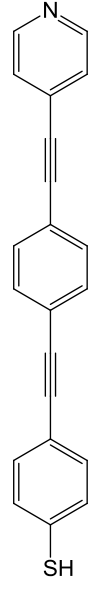

(18)

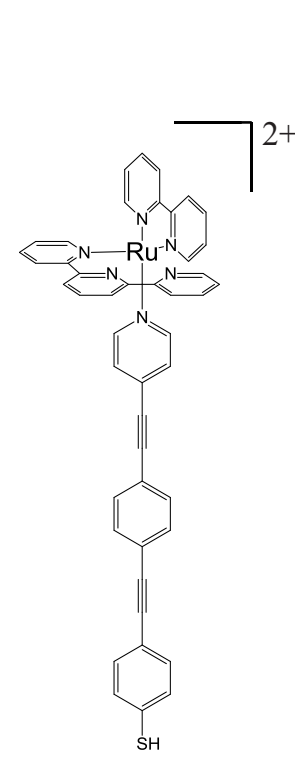

(19)

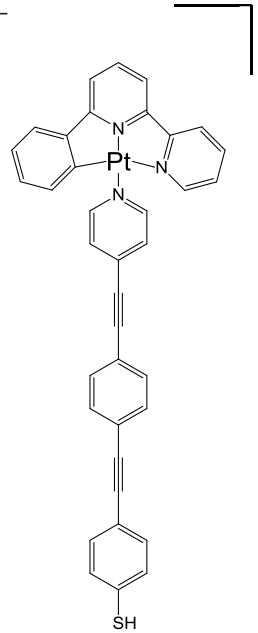

(20)

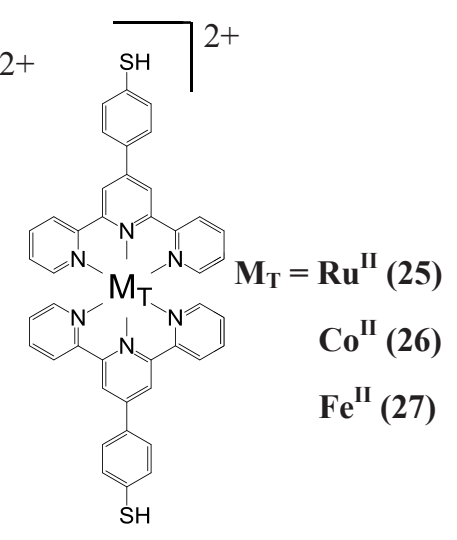

$(25$ - 27)

Figure 2.24: Molecular structure of Ru-complexed tripod thiol (16), Os-complexed tripod thiol (17), 1-((4acetylthiophenyl)ethynyl)-4-((4-pyridyl)ethynyl)benzene (18), Ru-complexed oligophenelyene ethylene thiol (19), Pt-complexed oligophenelyene ethylene thiol (20), Ru-complexed di-tridecanethiol (24), Ru-complexed di-benzenethiol (25), Co-complexed di-benzenethiol (26), Fe-complexed di-benzenethiol (27). 


\subsubsection{EGaIn Tunneling Junctions}

De Cola and co-workers investigated the charge transport characteristics of tripod-type SAMs terminated with luminescent ruthenium $(\mathrm{Ru})^{[58]}$ or osmium $(\mathrm{Os})^{[57]}$ complexes in EGaIn tunneling junctions (Figure 2.25). The authors performed statistically relevant numbers of $J(V)$ measurements $\pm 2.0 \mathrm{~V}$ across SAMs of Ru-complexed tripod thiol (16) and Os-complexed tripod thiol (17) on $\mathrm{Au}^{\mathrm{TS}}$, with a yield of working junctions of $82 \%$ and $100 \%$ respectively. SAMs of $\mathbf{1 7}$ were found to rectify currents at a negative bias with $R=32$ with a $\log$ standard deviation of 1.5 , calculated at $\pm 2.0 \mathrm{~V}$ (Figure $2.25 \mathrm{c}$ and d), whereas SAMs of 16 did not rectify currents $(R \sim 1.0)$ (Figure $2.25 \mathrm{a}$ and $\mathrm{b}$ ). The difference in charge transport characteristics is attributed to different energies of the HOMO levels of Ru and Os placed asymmetrically within the EGaIn tunneling junction, coupled with the $\left(\mathrm{Ga}_{2} \mathrm{O}_{3}\right) \mathrm{EGaIn}$ top electrode. The authors state that the HOMO level of Os in $\mathbf{1 7}$ is energetically closer to the Fermi level of the $\left(\mathrm{Ga}_{2} \mathrm{O}_{3}\right)$ EGaIn electrode than the HOMO level of $\mathrm{Ru}$ in $\mathbf{1 6}$ is. ${ }^{[57]}$ Therefore, the rectification in $\mathbf{1 7}$ is believed to occur due to the HOMO level of Os being in resonance with the Fermi levels of both electrodes at a lower applied potential at a negative bias, than at a positive bias. This then leads to the HOMO level of Os facilitating charge transport at lower applied potentials in one direction (negative bias), than the other (positive bias), thus causing rectification. The absence of rectification in $\mathbf{1 6}$ is believed to be due to the HOMO level of Ru not being in resonance with the Fermi levels of both electrodes at neither positive nor negative biases. 
a)

bulk

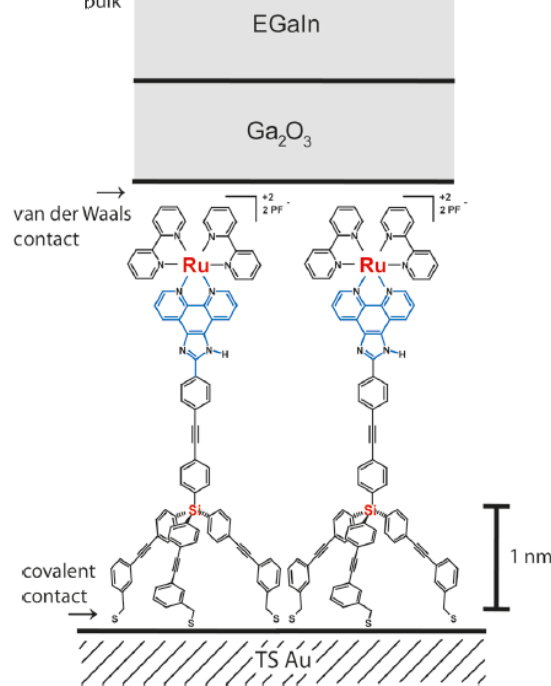

c)

c) bulk

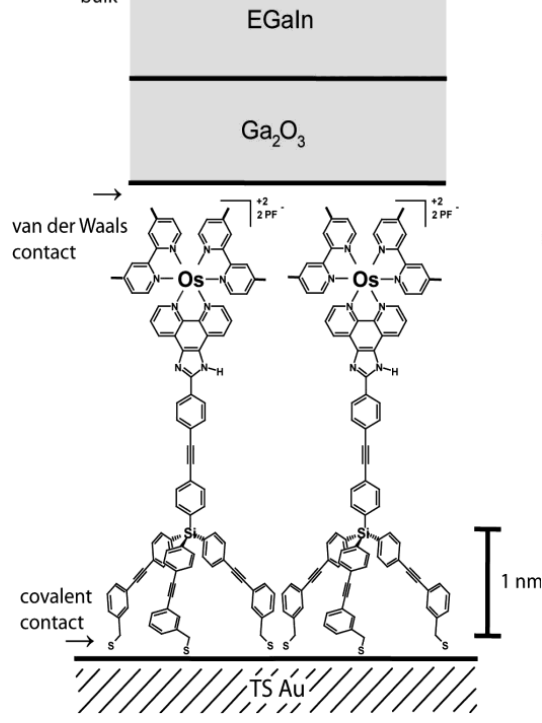

b)

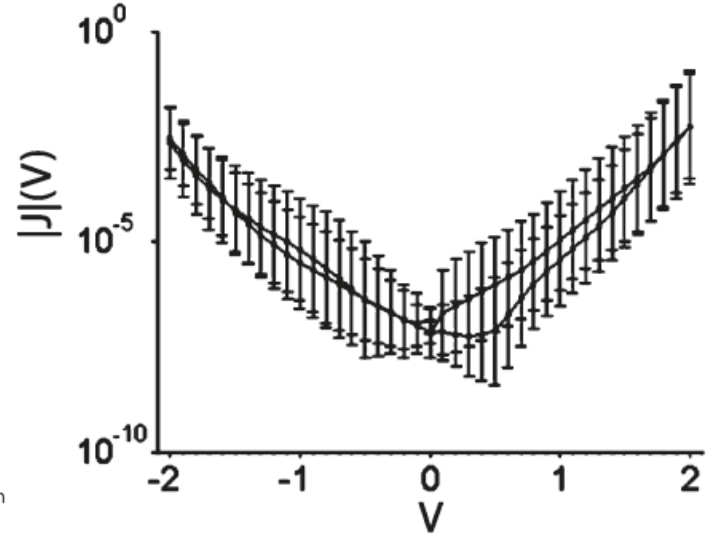

d)

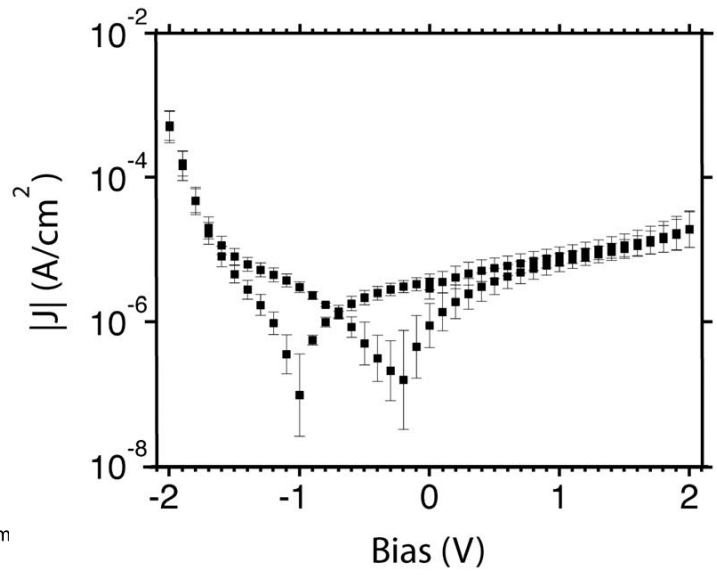

Figure 2.25: Schematic of a SAM of Ru-complexed tripod thiol (16) (a) and Os-complexed tripod thiol (17) (c) immobilized on a $\mathrm{Au}^{\mathrm{TS}}$ bottom electrode in EGaIn tunneling junctions. Semi-log plot of the averaged $|J|(V)$ measurements performed $\pm 2.0 \mathrm{~V}$, across SAMs of $\mathbf{1 6}$ (b) and $\mathbf{1 7}$ (d) in EGaIn tunneling junctions (Figures (a) and (b) reprinted with permission from. ${ }^{[58]}$ Copyright $(C)$ 2011, American Chemical Society, Figures (c) and (d) reprinted with permission from. ${ }^{[57]}$ Copyright $(\subset)$ 2011, IUPAC). 


\subsubsection{PEDOT:PSS Tunneling Junctions}

Yip and co-workers investigated the charge transport characteristics of SAMs of 1-((4acetylthiophenyl)ethynyl)-4-((4-pyridyl)ethynyl)benzene (18), Ru-complexed oligophenelyene ethylene thiol (19) and Pt-complexed oligophenelyene ethylene thiol (20) in PEDOT:PSS tunneling junctions at $\pm 1.0 \mathrm{~V}$ (Figure 2.26). ${ }^{[59]}$ Unlike other PEDOT:PSS tunneling junctions, these junctions contained a layer of chromium as an adhesion layer between the PEDOT:PSS layer and the $\mathrm{Au}$ top electrode. In the $J(V)$ measurements performed $\pm 1.0 \mathrm{~V}$, the SAM of $\mathbf{1 8}$ (black) slightly rectified currents at a negative bias, the SAM of $\mathbf{2 0}$ (dark blue) gave values of $J$ slightly higher/similar to that of $\mathbf{1 8}$ and slightly rectified currents at a positive bias. The SAM of 19 (light blue) gave values of $J$ approximately half an order of magnitude higher than that of $\mathbf{2 0}$ and also slightly rectified currents at a positive bias (Figure 2.26b). The rectification ratio $(R)$ calculated $(R=\mid J(-1.0$ $\mathrm{V})|/| J(+1.0 \mathrm{~V}) \mid)$ for SAMs of $\mathbf{2 0}$ and $\mathbf{1 9}$ was 2.25 and 2.4, respectively. The authors attributed the higher values of $J$ and the rectification at the positive bias for SAMs $\mathbf{2 0}$ and $\mathbf{1 9}$ to be due to the HOMO/LUMO levels of the metal complexes ( $\mathrm{Pt}$ and $\mathrm{Ru}$ ) being in resonance with the electrodes. However, the difference in the values of $J$ is small, the values of $R$ calculated are negligible and there is no explanation within the study whether statistically relevant numbers of data were accumulated. Therefore, no significant conclusions can be drawn from these data alone. A control $J(V)$ measurement performed $\pm 1.0 \mathrm{~V}$ on these PEDOT:PSS junctions in the absence of a SAM (red) did show values of $J$ higher than that of junctions containing the SAM, indicating that the $J(V)$ measurements were performed across the SAMs. Interestingly, these control $J(V)$ measurements also displayed a small amount of rectification at a positive bias, which further discredits the explanation of the data presented. Within this study, the $J(V)$ characteristics of these SAMs were also investigated using STM and CP-AFM, however, this is out of the scope of this review. 
a)

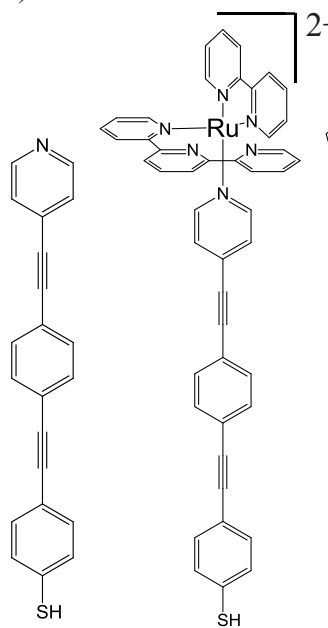

(18)

(19)

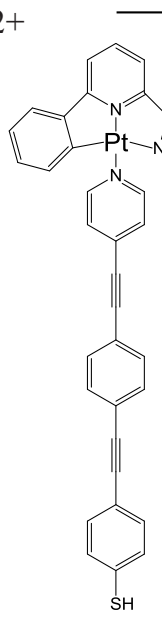

(20) b)

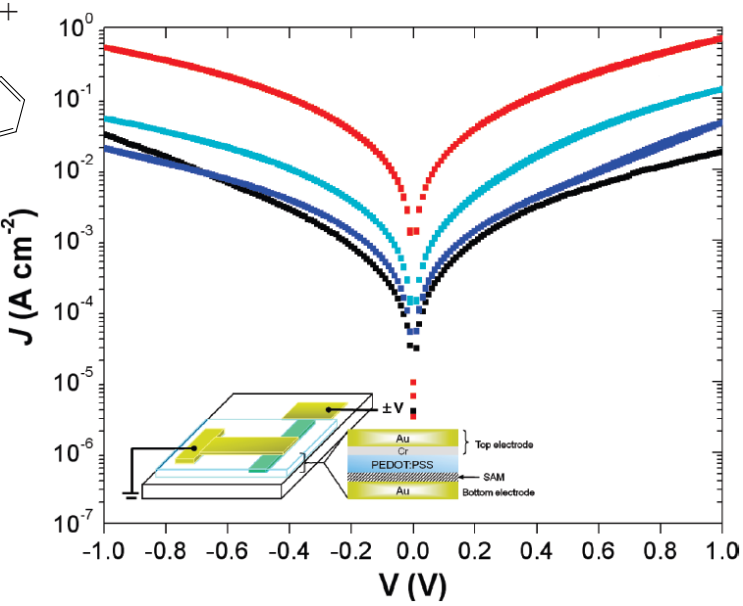

Figure 2.26: (a) Molecular structures of 1-((4-acetylthiophenyl)ethynyl)-4-((4-pyridyl)ethynyl)benzene (18), Ru-complexed oligophenelyene ethylene thiol (19) and Os-complexed oligophenelyene ethylene thiol (20). (b) Semi-log plot of the $J(V)$ measurements $\pm 1.0 \mathrm{~V}$ across SAMs of 18 (black), 20 (dark blue) and $\mathbf{1 9}$ (light blue) in PEDOT:PSS tunneling junctions with an additional chromium layer between the PEDOT:PSS layer and the top Au contact. Schematic of the PEDOT:PSS junction is shown within the Semi-log $J(V)$ plot (Figure (b) reprinted with permission from. ${ }^{[59]}$ Copyright $(C)$ 2009, American Chemical Society.)

Lee and coworkers investigated the charge transport characteristics of SAMs of dialkythiolate-tethered metal complexes in PEDOT:PSS tunneling junctions (Figure 2.27). ${ }^{[60]} I(V)$ measurements were performed $\pm 2.0 \mathrm{~V}$ across SAMs of Ru-complexed heptathiol (21), Ru-complexed tridecanethiol (22), Ru-complexed di-heptathiol (23) and Rucomplexed di-tridecanethiol (24), in PEDOT:PSS tunneling junctions with a diameter of $25 \mu \mathrm{m}^{2}$ and gave yields of working junctions of $0-4 \%$ for SAMs of 21 and 22, respectively, and $19 \%$ and $81 \%$ for SAMs of $\mathbf{2 3}$ and $\mathbf{2 4}$, respectively. The authors believe that SAMs of $\mathbf{2 4}$ displayed stable and reproducible molecular monolayer nonvolatile memory (MMNVM) as the $I(V)$ measurements displayed a small amount of hysteresis between $-1.0 \mathrm{~V}$ and $-2.0 \mathrm{~V}$ (Figure 2.27b) and voltage pulses performed at $-1.5 \mathrm{~V},-1.0 \mathrm{~V}$, $+1.5 \mathrm{~V}$ and $-1.0 \mathrm{~V}$, corresponding to writing, reading, erasing and reading voltages (WRER) respectively, were able to demonstrate that the junctions had clear high conducting ' $O N$ ' and low conducting 'OFF' states (Figure $2.27 \mathrm{c}$-e). However, the difference between the current measured for the 'ON' and 'OFF' state is relatively small and as PEDOT:PSS tunneling 54 
junctions have been shown to encounter difficulties at voltages of $\sim+2.0 \mathrm{~V}$ and $-2.0 \mathrm{~V},{ }^{[28]}$ the true origin of the nonvolatile memory may still be questionable.

a)

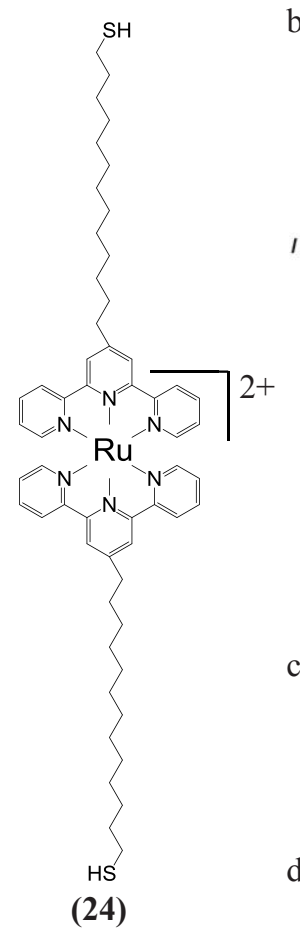

b)

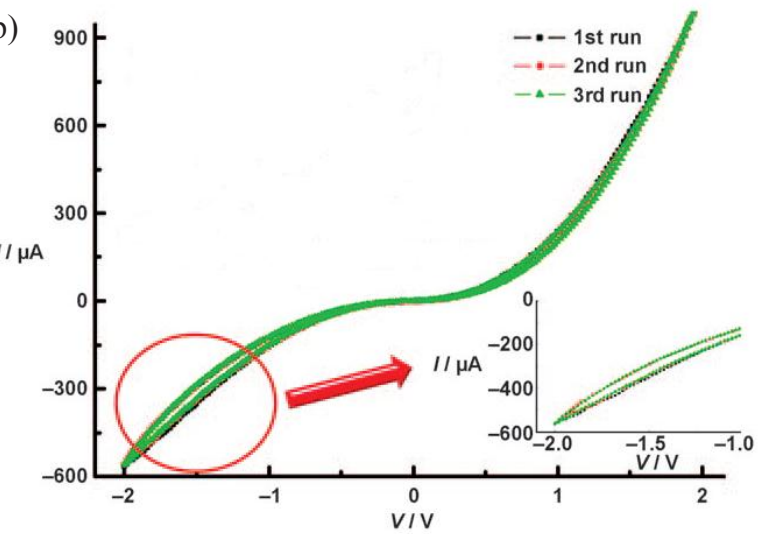

c)

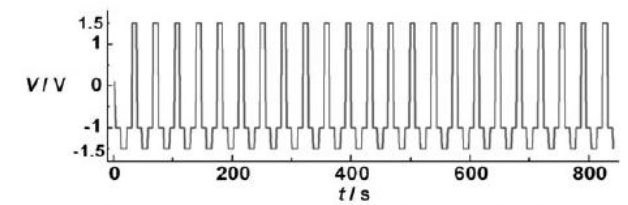

d)

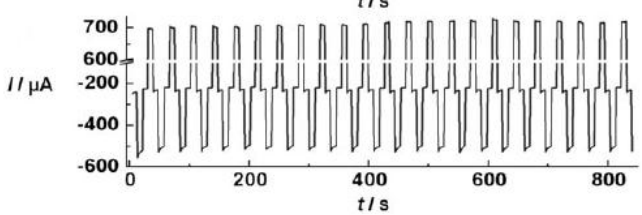

e)

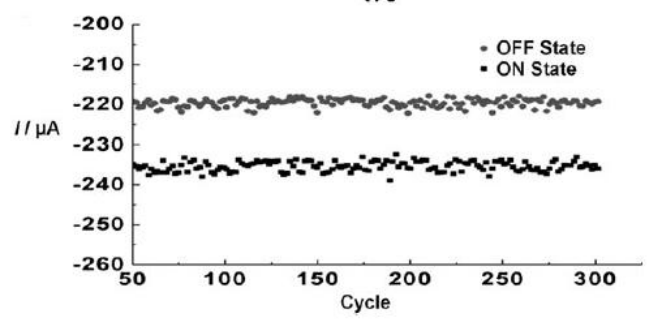

Figure 2.27: (a) Molecular structure of Ru-complexed di-tridecanethiol (24). (b) Linear $I(V)$ plot for measurement performed $\pm 2.0 \mathrm{~V}$ across the SAM of $\mathbf{2 4}$ in the PEDOT:PSS tunneling junction with the inset highlighting the hysteresis present. (c and d) Write-multiple read-erase-multiple read (WRER) cycles of SAM 24 in the PEDOT:PSS tunneling junction. (e) Current in the ON and OFF states as a function of the number of WRER cycles (Figures (b - e) reprinted with permission from. ${ }^{[60]}$ Copyright $(C)$ 2009, John Wiley and Sons). 


\subsubsection{Graphene Tunneling Junctions}

Lee and co-workers investigated the charge transport characteristics of SAMs of Rucomplexed di-benzenethiol (25), Co-complexed di-benzenethiol (26) and Fe-complexed dibenzenethiol (27) in rGO tunneling junctions (Figure 2.28). ${ }^{[21 b]}$ The $J(V)$ measurements performed $\pm 3.0 \mathrm{~V}$ across all three SAMs displayed hysteresis with the conductance switching between low-conducting and high-conducting states and therefore showed 'molecular memory' like properties (Figure $2.28 \mathrm{~b}$-d). These molecular memory properties were also examined by WRER operations. All SAMs displayed a retention time of at least 10 min for both the high-conducting state (ON) and the low conducting state (OFF), which is typically seen in nonvolatile memory. The authors propose that the conductance switching phenomena results from molecular resonant tunneling due to the energetic alignment of the molecular state with the Fermi level of the metal at a particular voltage. In addition the current hysteresis loop results from the switching between two states: the molecular charging energy at the high conductance state correlates to the energy difference between the electrochemical potential of the redox-active molecules and the metal's Fermi level. 
a)

$$
\begin{array}{r}
M_{T}=\operatorname{Ru}^{\text {II }}(25) \\
\operatorname{Co}^{\text {II }}(26) \\
\operatorname{Fe}^{\text {II }}(27)
\end{array}
$$

b)

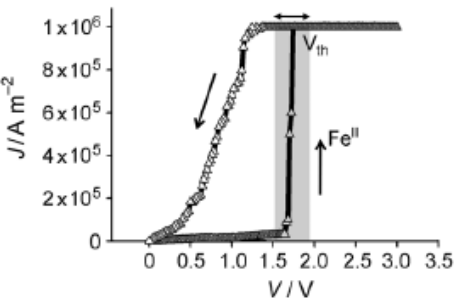

c)

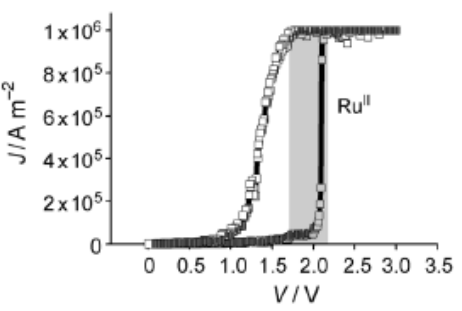

d)

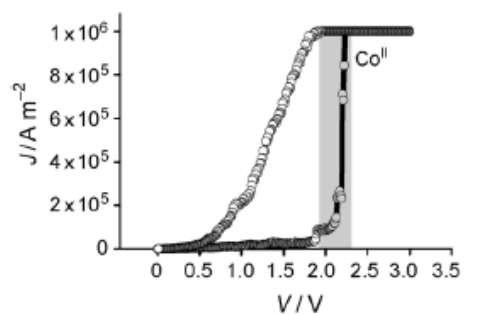

Figure 2.28: (a) Molecular structure of Ru-complexed di-benzenethiol (25), Co-complexed di-benzenethiol (26), and Fe-complexed di-benzenethiol (27). $I(V)$ measurements $0 \rightarrow 3.0 \mathrm{~V}$ across SAMs of 27 (b), 25 (c) and 26 (d), displaying the conductance switching (Figures $(b-d)$ reproduced with permission from. ${ }^{[21 b]}$ Copyright (c) 2011, John Wiley and Sons). 


\subsubsection{Photo-induced Electrical Switches}

The ability of some organic molecules to undergo a conformational change, upon the appropriate excitation with electromagnetic radiation, can be exploited in molecular tunneling junctions to create molecular switches. Typically, the conformational change originates from a $\pi$-conjugated molecular system being reversibly broken into a saturated aliphatic carbon chain, or vice versa, changing the efficiency of electron delocalization along the molecule (Figure 2.29). Below an example of this phenomena being exploited in PEDOT:PSS tunneling junctions is discussed.

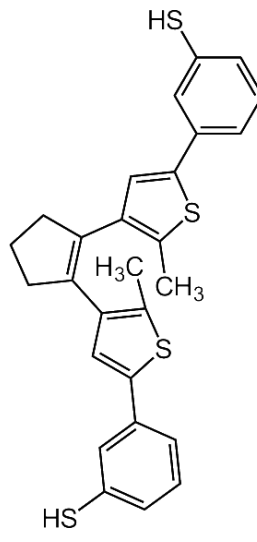

28

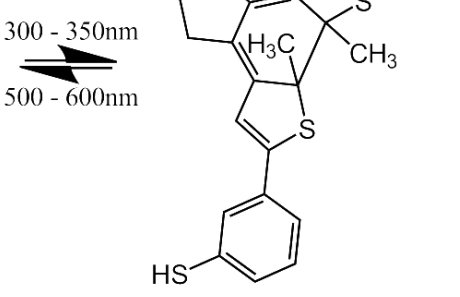

29

(conjugated closed form)

Figure 2.29: Molecular structures of the two isomers of the photochromic diarylethene: 3,3'(4,4'-(cyclopent-1ene-1,2-diyl)bis(5-methylthiophene-4,2-diyl))dibenzenethiol (non-conjugated) (28) and 3,3'-(9a,9b-dimethyl5,6,9a,9b-tetrahydro-4H-indeno[5,4-b:6,7-b’]dithiophene-2,8-diyl)dibenezenethiol (conjugated) (29).

\subsubsection{PEDOT:PSS Tunneling Junctions}

Conductance switching of a functional molecular monolayer based on the photochromic diarylethenes of 3,3'(4,4'-(cyclopent-1-ene-1,2-diyl)bis(5-methylthiophene-4,2diyl))dibenzenethiol (28) and 3,3'-(9a,9b-dimethyl-5,6,9a,9b-tetrahydro-4H-indeno[5,4$\left.b: 6,7-b^{\prime}\right]$ dithiophene-2,8-diyl)dibenezenethiol (29) has been shown by de Boer and coworkers in PEDOT:PSS tunneling junctions (Figure, 2.30). ${ }^{[30,}{ }^{61]}$. Photochromic 58 
diarylethenes can exist as one of two isomers. One is conjugated (29), whilst the other is non-conjugated (28), with it being possible to reversibly switch between both forms using either UV irradiation of 300 - $350 \mathrm{~nm}$ (non-conjugated to conjugated) or visible irradiation of $500-600 \mathrm{~nm}$ (conjugated to non-conjugated (28)). As discussed earlier in this review in section 2.3.2, conjugated (29) molecules have a smaller gap between their HOMO and LUMO level than non-conjugated (28) molecules. Therefore, when immobilized within a molecular tunneling junction, with the mechanism of charge transport being through-bond tunneling, the conjugated (29) isomer should facilitate charge transport better than the nonconjugated (28) isomer. de Boer and co-workers fabricated two separate sets of PEDOT:PSS tunneling junctions, both of which had thin semi-transparent top electrodes to allow light to pass through. ${ }^{[30,61]}$ One set of tunneling junctions contained SAMs of the conjugated (29) isomer, whilst the other contained SAMs of the non-conjugated (28) isomer. By performing statistically relevant numbers of $J(V)$ measurements at $\pm 0.75 \mathrm{~V}$, the authors found that the conjugated (29) SAM gave values of $J$ approximately 1 order of magnitude higher than that of the non-conjugated (28) SAM (Figure 2.30b) . Upon irradiating the non-conjugated (28) SAM with UV light of $312 \mathrm{~nm}$ for $15 \mathrm{~min}$, the authors found that the value of $J$ measured for the non-conjugated (28) SAM increased by 1 order of magnitude, and thus gave similar values of $J$ as the conjugated (29) SAM. This demonstrated that upon UV irradiation the non-conjugated (28) SAM 'switched' to the conjugated (29) SAM whilst immobilized within the PEDOT:PSS tunneling junction. In an attempt to reverse the switching process, the authors irradiated the now 'conjugated (29) SAM', with visible light of $532 \mathrm{~nm}$. Although, the value of $J$ measured did decrease, it decreased by only a factor of 3 , and thus the original value of $J$ measured for the non-conjugated (28) SAM was not re-obtained. To ensure that the change in $J$ measured was not due a small amount of heat created during the irradiation of the SAM, temperature dependent $J(V)$ measurements were performed across both the conjugated and non-conjugated SAM. As the $J(V)$ characteristics did not significantly change over the temperature range of $180-330 \mathrm{~K}$, the switching seen in the photochromic diarylethenes could be determined as molecular in origin.

Interestingly, when irradiating the set of tunneling junctions that were initially formed with the conjugated (29) SAM, with visible light of $532 \mathrm{~nm}$, there was no change in the value of $J$ measured. The authors hypothesized that the difference in switching phenomena could be due to two reasons. ${ }^{[30,61]}$ Firstly, that only a small percentage of the conjugated (29) 
molecule needs to be present within the SAM to dominate the $J$ measured, whereas a large percentage of non-conjugated (28) molecule needs to be present within the SAM to dominate $J$ measured. Therefore, only partial switching of the non-conjugated (28) molecule to the conjugated (29) molecule would allow $J$ to change quickly, whereas the necessary complete switching of the conjugated (29) molecule to the non-conjugated (28) molecule would cause $J$ to change much slower. Secondly, that the conjugated (29) molecule forms a more densely packed SAM than the non-conjugated (28) molecule. Therefore, the conjugated (29) molecule may be sterically hindered during the switching process, whereas the non-conjugated (28) molecule would not.

Additionally, the authors immobilized the photochromic diarylethene SAMs in 'strings', which are multiple junctions connected in series, that allow for 20, 40, 100 and even 200 junctions to be measured at once (Figure 2.30c). ${ }^{[30-31]}$ Interestingly, the switching $J(V)$ characteristics of the photochromic diarylethene SAMs measured in the single junctions were reproducible in the strings. 
a)
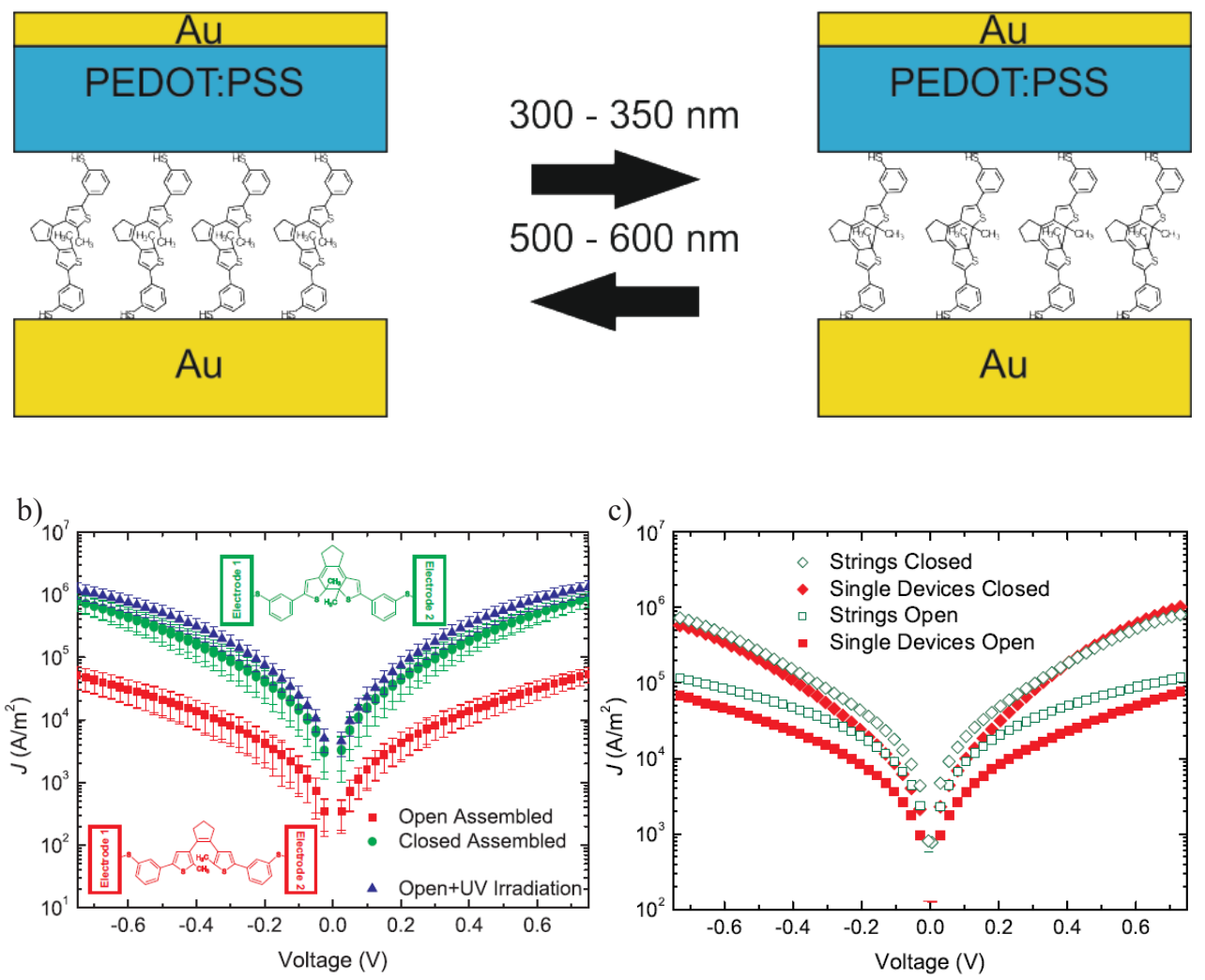

Figure 2.30: (a) Schematic of the photochromic diarylethene SAMs immobilized within the PEDOT:PSS tunneling junction, (left) non-conjugated (28) SAM, (right) conjugated SAM (29) (Figure re-drawn from references ${ }^{[30,61]}$ ). (b) Semi-log plot of the averaged $|J|(V)$ measurements performed $\pm 0.75 \mathrm{~V}$ across the conjugated (29, green) SAM and the non-conjugated (28, red) SAM of photochromic diarylethenes immobilized within the PEDOT:PSS tunneling junctions, and the $J(V)$ measurements of the PEDOT:PSS junctions where the non-conjugated (28) SAM was irradiated with UV light to the conjugated (29) SAM (blue). (c) Semi-log plot of the averaged $|J|(V)$ measurements performed $\pm 0.75 \mathrm{~V}$ across the photochromic diarylethene SAMs in strings and single junctions, with the strings displaying the same switching properties as the single junctions. For the strings, the voltage is calculated back to a single junction within the string. The data presented is an average of at least 35 single junctions and 14 strings, ranging from of 20-200 junctions in series. This allowed for over 1250 PEDOT:PSS junctions to be measured from a single fabrication batch (Figure (b) reproduced with permission from. ${ }^{[61]}$ Copyright (C) 2008, John Wiley and Sons. Figure (c) reproduced with permission from. ${ }^{[30]}$ Copyright (C) 2011, Auke Jisk Kronemeijer). 


\subsection{Conclusion and Outlook}

In this Chapter, the function and thus the $J(V)$ characteristics of various SAMs within a variety of large area molecular tunneling junctions were reviewed. Depending on the molecular characteristic of the SAM, the SAM functioned as either a dielectric (causing electrons to tunnel through the monolayer), semiconductor (causing the electrons to tunnel through the monolayer, however with less resistance than a dielectric layer), diode (causing a difference in the mechanism of charge transport at opposing biases i.e., hopping vs. tunneling, leading to rectification) or a switch (causing the current measured across the junction to change significantly).

Due to the previous complications and mis-interpretation of the $J(V)$ data in the field of molecular electronics, a majority of the results presented here were carefully analyzed and interpreted from statistically relevant numbers of data collected. However, even with this attention to detail, the data obtained for each SAM still varies depending on the molecular tunneling technique and, additionally, the way the measurements were performed. This is clearly seen in Table 3, where $\beta$ is given for alkanethiol (1) SAMs with an even numbered carbon chain and oligo-para-pheneylenethiol (9) SAMs in a variety of molecular tunneling junction architectures, with $\beta$ ranging from $0.57-0.90 \AA^{-1}$ for the alkanethiol SAMs and $0.20-0.61 \AA^{-1}$ for the oligo-para-pheneylenethiol SAMs and can also been seen in the variation of the $J(V)$ data of the three arylethylene thiol (7-9) SAMs discussed above in sections 2.3.2.1 and 2.3.2.2. This variation of $J(V)$ data also extends to small area and single molecule tunneling junctions as shown in a review by be Boer and co-workers, ${ }^{[3]}$ where $\beta$ was compared for alkanethiols and alkanedithiols in different tunneling junction techniques, ranging from single molecule STM measurements to large area tunneling junctions. Until there is a universal technique and method to investigate charge transport of SAMs in molecular tunneling junctions, these variations in $J(V)$ data are likely to still occur. Though with all of this said, the research groups within the field of molecular electronics are beginning to identify and understand the problems associated with the fabrication of working molecular tunneling junctions, and are openly acknowledging and discussing them in their subsequent publications. This is allowing for a possible collective group effort to solve these problems. However, is this enough to be able to produce molecular tunneling junctions that can rival or even be incorporated into the semiconducting industry? Only time will tell.

62 
Self-Assembled Monolayers in Large Area Molecular Tunneling Junctions

\begin{tabular}{|c|c|c|c|c|}
\hline SAM & Top Electrode & & $\beta\left(\AA^{-1}\right)$ & Reference \\
\hline & & Electrode & & \\
\hline Alkanethiol (1) & EGaIn & TS-Ag & 0.81 & 42 \\
\hline " & PEDOT:PSS & V-DEP-Au & 0.73 & $30,31,44$ \\
\hline " & Graphene (mGF) & V-DEP-Au & 0.85 & $21 \mathrm{a}$ \\
\hline " & Graphene (rGO) & V-DEP-Au & 0.82 & $21 b$ \\
\hline " & $\mathrm{Hg} / \mathrm{C}_{16} \mathrm{SH}$ & V-DEP-Ag & 0.87 & 45 \\
\hline$"$ & $\mathrm{Hg} / \mathrm{C}_{\mathrm{n}} \mathrm{SH}$ & TS-Ag & $0.57 / 0.64^{a}$ & $13 b$ \\
\hline $\bar{"}$ & $\mathrm{Hg} / \mathrm{C}_{\mathrm{n}} \mathrm{SH}$ & $\mathrm{Hg}$ & 0.68 & $22 \mathrm{c}$ \\
\hline$"$ & Complex (PmPV/Hg) & V-DEP-Au & 0.90 & 25 \\
\hline$"$ & $\mathrm{Au}$ & V-DEP-Au & $0.83^{\mathrm{b}}$ & 24 \\
\hline Oligo-para-pheneylenethiol (11) & PEDOT:PSS & V-DEP-Au & 0.20 & 30,49 \\
\hline " & $\mathrm{Hg} / \mathrm{C}_{16} \mathrm{SH}$ & V-DEP-Ag & 0.61 & 45 \\
\hline
\end{tabular}

Table 3: Values of $\beta$ for alkanethiol (1) SAMs with an even numbered carbon chain, and oligo-parapheneylenethiol (11) SAMs in a variety of molecular tunneling junctions architectures. ${ }^{\mathrm{a}}=$ two values are given as two different methods of data analysis was used, ${ }^{b}=$ an average value of $\beta$ was calculate from $\beta$ given for each individual alkanethiol molecule.

In this thesis the construction of the EGaIn tunneling technique in our laboratory is described. This technique is used to characterize the $J(V)$ characteristics of a supramolecular platform on which dendrimers of different terminal functionalities, core type and generation can be immobilized, to create well-defined structures. These studies allowed for the formulation of a hypothesis about the mechanism of charge transport within the supramolecular structure and additionally define a previously unknown limitation of the EGaIn technique. Finally, the charge transport characteristics of the supramolecular platform at the single molecule level are investigated, by performing Scanning Tunneling Microscopy measurements. 


\subsection{References}

[1] a)G. M. Whitesides, M. Boncheva, Proc. Natl. Acad. Sci. U. S. A. 2002, 99, 4769-4774; b)G. M. Whitesides, Sci.Am. 1995, 273, 146-149; c)G. M. Whitesides, B. Grzybowski, Science 2002, 295, 2418-2421.

[2] J. C. Love, L. A. Estroff, J. K. Kriebel, R. G. Nuzzo, G. M. Whitesides, Chem. Rev. 2005, 105, 1103-1169

[3] R. L. McCreery, A. J. Bergren, Adv. Mater. 2009, 21, 4303-4322.

[4] a)H. Song, M. A. Reed, T. Lee, Adv. Mater. 2011, 23, 1583-1608; b)F. Chen, J. Hihath, Z. F. Huang, X. L. Li, N. J. Tao, in Annu. Rev. Phys. Chem., Vol. 58, Annual Reviews, Palo Alto, 2007, pp. 535-564.

[5] a)L. A. Bumm, J. J. Arnold, T. D. Dunbar, D. L. Allara, P. S. Weiss, J. Phys. Chem. B 1999, 103, 8122-8127; b)X. D. Cui, A. Primak, X. Zarate, J. Tomfohr, O. F. Sankey, A. L. Moore, T. A. Moore, D. Gust, G. Harris, S. M. Lindsay, Science 2001, 294, 571-574; c)A.-S. Hallbäck, B. Poelsema, H. J. W. Zandvliet, Solid State Commun. 2007, 141, 645-648; d)B. Venkataraman, J. J. Breen, G. W. Flynn, Scanning Tunneling Microscopy Studies of Alcohol Alkane Mixtures Adsorbed on Graphite Surfaces, Plenum Press Div Plenum Publishing Corp, New York, 1994.

[6] a)D. J. Wold, C. D. Frisbie, J. Am. Chem. Soc. 2001, 123, 5549-5556; b)V. B. Engelkes, J. M. Beebe, C. D. Frisbie, J. Am. Chem. Soc. 2004, 126, 14287-14296; c)G. Wang, T. W. Kim, G. Jo, T. Lee, J. Am. Chem. Soc. 2009, 131, 5980-5985; d)C. M. Guedon, H. Valkenier, T. Markussen, K. S. Thygesen, J. C. Hummelen, S. J. van der Molen, Nature Nanotech. 2012, 7, 304-308.

[7] a)T. Lee, W. Y. Wang, J. F. Klemic, J. J. Zhang, J. Su, M. A. Reed, J. Phys. Chem. B 2004, 108, 8742-8750; b)M. A. Reed, C. Zhou, C. J. Muller, T. P. Burgin, J. M. Tour, Science 1997, 278, 252-254; c)W. Y. Wang, T. Lee, M. A. Reed, J. Phys. Chem. B 2004, 108, 18398-18407.

[8] a)J. G. Kushmerick, D. B. Holt, J. C. Yang, J. Naciri, M. H. Moore, R. Shashidhar, Phys. Rev. Lett. 2002, 89, 086802; b)J. G. Kushmerick, D. B. Holt, S. K. Pollack, M. A. Ratner, J. C. Yang, T. L. Schull, J. Naciri, M. H. Moore, R. Shashidhar, J. Am. Chem. Soc. 2002, 124, 10654 10655.

[9] a)C. Zhou, M. R. Deshpande, M. A. Reed, L. Jones li, J. M. Tour, Appl. Phys. Lett. 1997, 71, 611-613; b)J. Chen, M. A. Reed, A. M. Rawlett, J. M. Tour, Science 1999, 286, 1550-1552.

[10] a)I. Levine, S. M. Weber, Y. Feldman, T. Bendikov, H. Cohen, D. Cahen, A. Vilan, Langmuir 2012, 28, 404-415; b)F. Thieblemont, O. Seitz, A. Vilan, H. Cohen, E. Salomon, A. Kahn, D. 
Cahen, Adv. Mater. 2008, 20, 3931-3936; cY. Selzer, A. Salomon, D. Cahen, J. Am. Chem. Soc. 2002, 124, 2886-2887.

[11] a)A. P. Bonifas, R. L. McCreery, Nature Nanotech. 2010, 5, 612-617; b)R. L. McCreery, J. Wu, R. P. Kalakodimi, Phys. Chem. Chem. Phys. 2006, 8, 2572-2590; c)A. J. Bergren, K. D. Harris, F. Deng, R. McCreery, J. Phys.: Condens. Matter 2008, 20, 374117.

[12] a)R. M. Metzger, B. Chen, U. Hopfner, M. V. Lakshmikantham, D. Vuillaume, T. Kawai, X. Wu, H. Tachibana, T. V. Hughes, H. Sakurai, J. W. Baldwin, C. Hosch, M. P. Cava, L. Brehmer, G. J. Ashwell, J. Am. Chem. Soc. 1997, 119, 10455-10466; b)W. J. Shumate, D. L. Mattern, A. Jaiswal, D. A. Dixon, T. R. White, J. Burgess, A. Honciuc, R. M. Metzger, J. Phys. Chem. $B$ 2006, 110, 11146-11159.

[13] a)E. A. Weiss, J. K. Kriebel, M. A. Rampi, G. M. Whitesides, Philos. Trans. R. Soc. A-Math. Phys. Eng. Sci. 2007, 365, 1509-1537; b)E. A. Weiss, R. C. Chiechi, G. K. Kaufman, J. K. Kriebel, Z. Li, M. Duati, M. A. Rampi, G. M. Whitesides, J. Am. Chem. Soc. 2007, 129, 43364349.

[14] P. E. Laibinis, G. M. Whitesides, D. L. Allara, Y. T. Tao, A. N. Parikh, R. G. Nuzzo, J. Am. Chem. Soc. 1991, 113, 7152-7167.

[15] E. A. Weiss, G. K. Kaufman, J. K. Kriebel, Z. Li, R. Schalek, G. M. Whitesides, Langmuir 2007, 23, 9686-9694.

[16] M. H. Dishner, M. M. Ivey, S. Gorer, J. C. Hemminger, F. J. Feher, J. Vac. Sci. Technol. A-Vac. Surf. Films 1998, 16, 3295-3300.

[17] a)D. Q. Gao, F. Scholz, H. G. Nothofer, W. E. Ford, U. Scherf, J. M. Wessels, A. Yasuda, F. von Wrochem, J. Am. Chem. Soc. 2011, 133, 5921-5930; b)D. Losic, J. G. Shapter, J. J. Gooding, Langmuir 2001, 17, 3307-3316.

[18] R. C. Chiechi, E. A. Weiss, M. D. Dickey, G. M. Whitesides, Angew. Chem. Int. Ed. 2008, 47, 142-144.

[19] M. D. Dickey, R. C. Chiechi, R. J. Larsen, E. A. Weiss, D. A. Weitz, G. M. Whitesides, Adv. Funct. Mater. 2008, 18, 1097-1104.

[20] H. B. Akkerman, P. W. M. Blom, D. M. de Leeuw, B. de Boer, Nature 2006, 441, 69-72.

[21] a)G. Wang, Y. Kim, M. Choe, T. W. Kim, T. Lee, Adv. Mater. 2011, 23, 755-760; b)S. Seo, M. Min, J. Lee, T. Lee, S.-Y. Choi, H. Lee, Angew. Chem. Int. Ed. 2012, 51, 108-112.

[22] a)K. Slowinski, R. V. Chamberlain, C. J. Miller, M. Majda, J. Am. Chem. Soc. 1997, 119, 11910-11919; b)M. A. Rampi, O. J. A. Schueller, G. M. Whitesides, Appl. Phys. Lett. 1998, 72, 1781-1783; c)K. Slowinski, H. K. Y. Fong, M. Majda, J. Am. Chem. Soc. 1999, 121, 7257-7261; 
d)R. Haag, M. A. Rampi, R. E. Holmlin, G. M. Whitesides, J. Am. Chem. Soc. 1999, 121, 78957906; e)K. Slowinski, M. Majda, J. Electroanal. Chem. 2000, 491, 139-147.

[23] M. A. Rampi, G. M. Whitesides, Chem. Phys. 2002, 281, 373-391.

[24] T.-W. Kim, G. Wang, H. Lee, T. Lee, Nanotechnology 2007, 18, 315204.

[25] F. Milani, C. Grave, V. Ferri, P. Samori, M. A. Rampi, ChemPhysChem 2007, 8, 515-518.

[26] E. A. Speets, B. J. Ravoo, F. J. G. Roesthuis, F. Vroegindeweij, D. H. A. Blank, D. N. Reinhoudt, Nano Lett. 2004, 4, 841-844.

[27] C. A. Nijhuis, W. F. Reus, G. M. Whitesides, J. Am. Chem. Soc. 2009, 131, 17814-17827.

[28] B. C. de Brito, E. C. P. Smits, P. A. van Hal, T. C. T. Geuns, B. de Boer, C. J. M. Lasance, H. L. Gomes, D. M. de Leeuw, Adv. Mater. 2008, 20, 3750-3753.

[29] a)A. J. Kronemeijer, E. H. Huisman, I. Katsouras, P. A. van Hal, T. C. T. Geuns, P. W. M. Blom, S. J. van der Molen, D. M. de Leeuw, Phys. Rev. Lett. 2010, 105, 156604; b)A. J. Kronemeijer, I. Katsouras, E. H. Huisman, P. A. van Hal, T. C. T. Geuns, P. W. M. Blom, D. M. de Leeuw, Small 2011, 7, 1593-1598.

[30] A. J. Kronemeijer, PhD Thesis, University of Groningen, The Netherlands 2011. ISBN: 97890-367-4845-2

[31] P. A. Van Hal, E. C. P. Smits, T. C. T. Geuns, H. B. Akkerman, B. C. De Brito, S. Perissinotto, G. Lanzani, A. J. Kronemeijer, V. Geskin, J. Cornil, P. W. M. Blom, B. De Boer, D. M. De Leeuw, Nature Nanotech. 2008, 3, 749-754.

[32] R. L. McCreery, Chem. Mater. 2004, 16, 4477-4496.

[33] H. B. Akkerman, B. de Boer, J. Phys.: Condens. Matter 2008, 20, 013001.

[34] a)J. M. Seminario, L. Yan, Int. J. Quantum Chem 2005, 102, 711-723; b)C.-C. Kaun, H. Guo, Nano Lett. 2003, 3, 1521-1525.

[35] G. Wang, T. W. Kim, H. Lee, T. Lee, Phys. Rev. B 2007, 76, 205320.

[36] a)X. Y. Zhu, J. Phys. Chem. B 2004, 108, 8778-8793; b)Y. Imry, R. Landauer, Rev. Mod. Phys. 1999, 71, S306-S312; c)A. Salomon, D. Cahen, S. Lindsay, J. Tomfohr, V. B. Engelkes, C. D. Frisbie, Adv. Mater. 2003, 15, 1881-1890.

[37] a)W. Y. Wang, T. Lee, M. A. Reed, Phys. Rev. B 2003, 68, 035416; b)J. G. Simmons, J. Appl. Phys. 1963, 34, 1793-1803.

[38] a)A. Aviram, M. A. Ratner, Chem. Phys. Lett. 1974, 29, 277-283; b)R. Liu, S. H. Ke, W. T. Yang, H. U. Baranger, J. Chem. Phys. 2006, 124, 024718; c)P. E. Kornilovitch, A. M. Bratkovsky, R. S. Williams, Phys. Rev. B 2002, 66, 165436; d)N. Armstrong, R. C. Hoft, A. McDonagh, M. B. Cortie, M. J. Ford, Nano Lett. 2007, 7, 3018-3022. 
[39] a)Q. Lu, K. Liu, H. Zhang, Z. Du, X. Wang, F. Wang, ACS Nano 2009, 3, 3861-3868; b)S. H. Choi, C. Risko, M. C. R. Delgado, B. Kim, J. L. Bredas, C. D. Frisbie, J. Am. Chem. Soc. 2010, 132, 4358-4368; c)S. H. Choi, B. Kim, C. D. Frisbie, Science 2008, 320, 1482-1486.

[40] C. Joachim, M. A. Ratner, Proc. Natl. Acad. Sci. U. S. A. 2005, 102, 8801-8808.

[41] a)C. A. Nijhuis, W. F. Reus, G. M. Whitesides, J. Am. Chem. Soc. 2010, 132, 18386-18401; b)C. A. Nijhuis, W. F. Reus, J. R. Barber, M. D. Dickey, G. M. Whitesides, Nano Lett. 2010, 10, 3611-3619.

[42] M. M. Thuo, W. F. Reus, C. A. Nijhuis, J. R. Barber, C. Kim, M. D. Schulz, G. M. Whitesides, J. Am. Chem. Soc. 2011, 133, 2962-2975.

[43] H. B. Akkerman, A. J. Kronemeijer, P. A. van Hal, D. M. de Leeuw, P. W. M. Blom, B. de Boer, Small 2008, 4, 100-104.

[44] H. B. Akkerman, PhD Thesis, University of Groningen, The Netherlands, 2008. ISBN: 978-90$367-4845-2$

[45] a)R. E. Holmlin, R. Haag, M. L. Chabinyc, R. F. Ismagilov, A. E. Cohen, A. Terfort, M. A. Rampi, G. M. Whitesides, J. Am. Chem. Soc. 2001, 123, 5075-5085; b)R. E. Holmlin, F. I. Rustem, H. Rainer, M. Vladimiro, A. R. Mark, R. Maria Anita, M. W. George, Angew. Chem. Int. Ed. 2001, 40, 2316-2320.

[46] a)R. L. York, K. Slowinski, J. Electroanal. Chem. 2003, 550, 327-336; b)R. L. York, P. T. Nguyen, K. Slowinski, J. Am. Chem. Soc. 2003, 125, 5948-5953.

[47] G. Wang, T.-W. Kim, Y. H. Jang, T. Lee, J. Phys. Chem. C 2008, 112, 13010-13016.

[48] D. Vuillaume, Vol. 3, Oxford University Press, 2010, pp. 312-342.

[49] A. J. Kronemeijer, E. H. Huisman, H. B. Akkerman, A. M. Goossens, I. Katsouras, P. A. van Hal, T. C. T. Geuns, S. J. van der Molen, P. W. M. Blom, D. M. de Leeuw, Appl. Phys. Lett. 2010, 97, 173302.

[50] D. Fracasso, H. Valkenier, J. C. Hummelen, G. C. Solomon, R. C. Chiechi, J. Am. Chem. Soc. 2011, 133, 9556-9563.

[51] H. Liu, N. Wang, J. Zhao, Y. Guo, X. Yin, F. Y. C. Boey, H. Zhang, ChemPhysChem 2008, 9, 1416-1424.

[52] M. Kondo, T. Tada, K. Yoshizawa, J. Phys. Chem. A 2004, 108, 9143-9149.

[53] Information obtained from a conversation with Hennie Valkenier.

[54] a)H. V. H. Valkenier, E. H. Huisman, P. A. van Hal, D. M. de Leeuw, R. C. Chiechi, J. C. Hummelen, J. Am. Chem. Soc. 2011, 133, 4930-4939; b)H. Valkenier, PhD Thesis, University of Groningen, The Netherlands 2011. ISBN: 978-90-367-4943-5 
[55] C. Grave, C. Risko, A. Shaporenko, Y. L. Wang, C. Nuckolls, M. A. Ratner, M. A. Rampi, M. Zharnikov, Adv. Funct. Mater. 2007, 17, 3816-3828.

[56] C. A. Nijhuis, W. F. Reus, A. C. Siegel, G. M. Whitesides, J. Am. Chem. Soc. 2011, 133, 1539715411.

[57] S. Ramachandra, F. Polo, F. Edafe, K. C. Schuermann, C. A. Nijhuis, P. Belser, W. F. Reus, G. M. Whitesides, L. De Cola, Pure Appl. Chem. 2011, 83, 779-799.

[58] S. Ramachandra, K. C. Schuermann, F. Edafe, P. Belser, C. A. Nijhuis, W. F. Reus, G. M. Whitesides, L. De Cola, Inorg. Chem. 2011, 50, 1581-1591.

[59] Z. Y. Ng, K. P. Loh, L. Q. Li, P. Ho, P. Bai, J. H. K. Yip, ACS Nano 2009, 3, 2103-2114.

[60] J. Lee, H. Chang, S. Kim, G. S. Bang, H. Lee, Angew. Chem. Int. Ed. 2009, 48, 8501-8504.

[61] A. J. Kronemeijer, H. B. Akkerman, T. Kudernac, B. J. van Wees, B. L. Feringa, P. W. M. Blom, B. de Boer, Adv. Mater. 2008, 20, 1467-1473. 


\section{Chapter 3}

\section{The EGaIn Technique}

The use of eutectic gallium indium (EGaIn) to contact self-assembled monolayers (SAMs) has been able to address a major shortcoming, i.e. the top contact electrode, of the molecular electronics field. The EGaIn technique developed by Whitesides and co-workers, forms stable, reproducible, 'high' yielding molecular tunneling junctions that allow for the accumulation of statistically relevant numbers of data. As using this technique in the laboratories of Whitesides and co-workers was highly successful and as the components used to construct this technique are relatively cheap and easy to assemble, a similar setup was also built at the University of Twente. Discussed in this chapter are the construction of the apparatus, including all parts ordered and assembled, and the calibration measurements performed. The advantages and disadvantages of the EGaIn technique are compared with the other techniques currently available to investigate charge transport across SAMs in tunneling junctions. 


\subsection{Introduction}

A variety of techniques has been developed to investigate charge transport across SAMbased tunneling junctions. ${ }^{[1]}$ In 2008 Whitesides and co-workers developed a technique where the fluid metal eutectic gallium-indium (which they abbreviated to "EGaIn", and pronounce as a coined term "egain"; $75.5 \% \mathrm{Ga}, 24.5 \% \mathrm{In}$ by weight, $15.7{ }^{\circ} \mathrm{C}$ melting point) is used as the top contact to address SAMs in molecular tunneling junctions. ${ }^{[2]}$ The "EGaIn technique" is able to address the difficulty in forming stable, reproducible, molecular tunneling junctions in high yields, allowing for statistically relevant numbers of data to be accumulated. ${ }^{[2-3]}$ Using the EGaIn technique in the laboratories of Whitesides and coworkers allowed for the investigation of charge transport characteristics of supramolecular tunneling junctions. Due to the successful nature of these studies (Chapter 4), and minimal expense to construct this technique, the EGaIn technique was re-constructed at the University of Twente. This allowed for the further investigation of the charge transport characteristics of various supramolecular tunneling junctions (Chapter 5) and the EGaIn technique itself (Chapter 6).

First in this Chapter, the different techniques used to create two-terminal large-area SAM based tunneling junctions will be discussed along with their advantages and disadvantages. The scope of this discussion will exclude techniques used to create small-area $(<1 \mu \mathrm{m}$ in diameter) SAM tunneling junctions, ${ }^{[1 \mathrm{a}, 4]}$ such as, scanning tunneling microscopy $(\mathrm{STM})^{[5]}$ conductive probe atomic force microscopy $(\mathrm{CP}-\mathrm{AFM}),{ }^{[6]}$ break junctions, ${ }^{[7]}$ crossed wire junctions ${ }^{[8]}$ and nanopores. ${ }^{[9]}$ This will be followed by a detailed account of the construction of the EGaIn technique at the University of Twente, including all materials used and calibration measurements performed, along with how to create an EGaIn tunneling junction and subsequently perform $I(V)$ measurements. Finally, discussed will be the importance of the accumulation of statistically relevant numbers of data, and how to analyze all of the data.

There is not one universal technique to investigate the $I(V)$ characteristics of SAMs in largearea tunneling junctions. ${ }^{[1 \mathrm{~b}, 2,10]}$ The most common two-terminal large-area SAM based tunneling junction techniques that have been or are being used to perform $I(V)$ measurements are, metal evaporation techniques, ${ }^{[10 \mathrm{a}, 10 \mathrm{~b}]}$ metal/nano transfer printing (soft contact deposition) ${ }^{[10 \mathrm{c}, 10 \mathrm{~d}, 10 \mathrm{~h}]}$ graphene interlayer electrode ${ }^{[10 \mathrm{i}, 10 \mathrm{j}]}$ hanging mercury drop

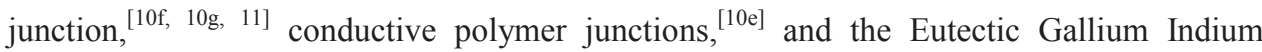
technique ${ }^{[2]}$. Below these will be discussed separately. 
Originally, the technique of choice to fabricate molecular tunneling junctions was to create top contacts of a well-defined size by thermal evaporation of metals through a shadow mask directly onto the SAM. ${ }^{[10 a, 10 b]}$ This method made it possible to use the same metal for both the bottom and top electrode (i.e. both metal electrodes will then have the same work function), and allows for the formation of a chemisorbed contact at both electrode-molecule interfaces. However, this technique has been found to have severe drawbacks. Studies carried out in our group (Figure 3.1b), ${ }^{[12]}$ and by Lee and co-workers, ${ }^{[10 b]}$ demonstrated how destructive metal evaporation onto the SAM can be and that even when carefully prepared, junctions created by this method give low yields $(<5 \%)$ of working junctions. This is due to the evaporated metals forming metal filaments within the SAM (Figure 3.1a), damaging the organic molecules and possibly forming metal oxides giving rise to such phenomena as Schottky barriers and causing 'ill-defined' chemical interactions with the SAM. Therefore, the $I(V)$ characteristics of metal evaporated tunneling junctions may be dominated by the metal filaments and/or metal oxides (i.e. being the origin of switching and rectification), making it difficult to study the mechanism of charge transport of the SAM itself.

a)

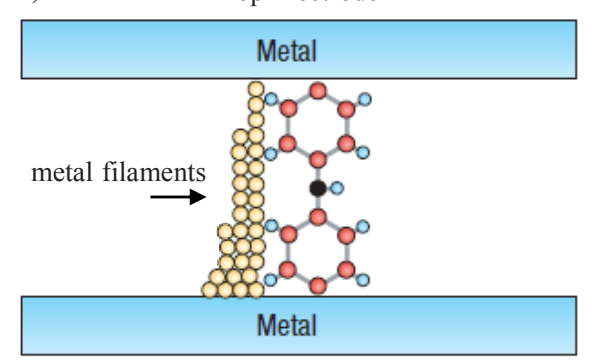

Bottom Electrode b)

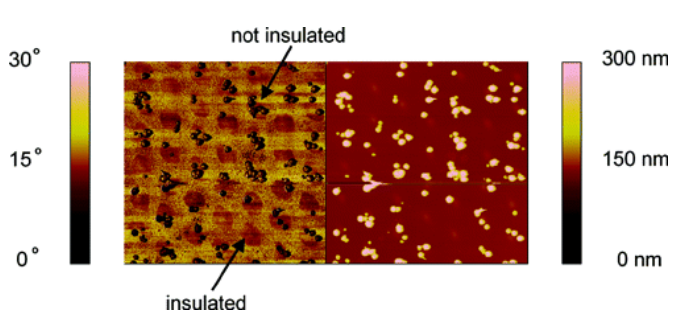

Figure 3.1: (a) Schematic of a molecular tunneling junction where the top electrode was fabricated by thermal evaporation of the metal directly onto the SAM, with metal filaments within the molecular layer, bridging the top and bottom electrode, causing an electrical short (Figure reprinted by permission from Macmillan Publishers Ltd: Nature, ${ }^{[13]}$ Copyright $($ 2006). (b) AFM phase and height images of a SAM of octadecanethiol $\left(\mathrm{C}_{18} \mathrm{SH}\right)$ on $\mathrm{Au}$ after subsequent pulsed laser deposition of $\mathrm{Au}$ islands and electrochemical copper deposition (Scale of AFM scans: $10 \times 10 \mu \mathrm{m}$, Figure reprinted with permission from. ${ }^{[12]}$ Copyright $(\subset)$ 2004, American Chemical Society). 
The technique nano-transfer printing (nTP) ${ }^{[10 \mathrm{~h}, 14]}$ was developed to overcome the problems associated with the direct evaporation of metals onto the SAM, whilst still holding true the technique's original advantages. The metal was evaporated onto a pre-patterned polymer based stamp (such as polydimethylsiloxane (PDMS) or perfluoropolyether (PFPE) molded on and removed from a patterned silicon wafer), and then brought into contact with the SAM (commonly an alkanedithiol) by hand. After a specified period of time the stamp was removed, transferring the patterned metal film from the stamp to the SAM due to the chemical $\mathrm{Au}-\mathrm{S}$ interaction being stronger than the interaction of the Au with the PDMS stamp (Figure 3.2). Although innovative, this technique also has several drawbacks. It is a lengthy process which involves costly cleanroom procedures (i.e. creating silicon wafer masters for patterning the PDMS stamps). Placing the metal coated stamps onto the SAM by hand creates an undefined amount of force on the SAM that will vary from device to device. The type of SAM investigated and metal used is limited as a strong interaction between the metal and molecule is required to remove the metal film from the PDMS stamp. Finally, a low yield of working devices is suspected (and can only be suspected as there is no study stating the yields of $I(V)$ measurements on tunneling junctions created using this technique) as a study carried out by Rogers and co-workers ${ }^{[14]}$ speculates that $>15 \%$ of the top contacts are transferred successfully. Therefore $<20 \%$ of the devices were successfully fabricated, with the yield surely decreasing upon $I(V)$ investigation.

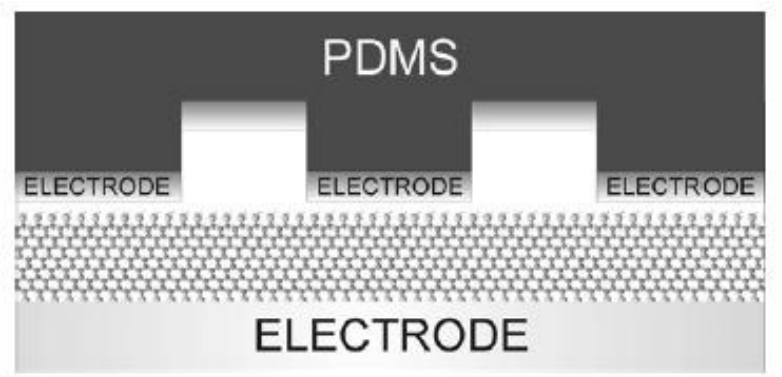

Figure 3.2: Schematic representation of the top electrodes being fabricated using the nano-transfer printing (nTP) procedure (Figure reprinted with permission from. ${ }^{[1 \mathrm{c}]}$ Copyright $(C)$ 2008, IOPscience).

Additional 'soft contact deposition' techniques such as the lift-off-float-on (LOFO) process $^{[10 \mathrm{c}]}$ and the polymer-assisted lift-off (PALO) process ${ }^{[10 \mathrm{~d}]}$ have also been developed to improve on the problems associated with creating top contacts via metal evaporation and in some cases nTP. The LOFO process entails detaching a thin metal film from a suitable 72 
solid support in a specific liquid (Figure 3.3). Detachment readily occurs when the interaction between the metal film and solid support is weak (i.e. glass and Au), however, if the interaction is strong, a 'release layer' that can be selectively etched, is inserted in-between the two materials, allowing for the detachment of the metal film. As the metal film begins to detach itself, the solid support is dipped into a 'clean suitable solvent', allowing the metal film to float on the surface. Removal of the solvent allows the metal film to be lowered onto a SAM formed on a pre-patterned metal electrode. The PALO process is very similar to the LOFO process. It entails an additional step where a backing polymer layer is added on top of a solid support containing patterned metal electrodes. The patterned metal electrodes along with the polymer are both detached from the solid support upon immersion into a suitable solvent.

The drawbacks of these procedures are that they are both lengthy and complicated (i.e. removal of all liquid from the device requires drying under vacuum for hours) and may require expensive clean room procedures to obtain patterned metal electrodes. ${ }^{[10 \mathrm{c}, 10 \mathrm{~d}]}$ The SAM-metal combinations are limited by repulsion forces (i.e. hydrophilicity and hydrophobicity) between the SAM and floating metal film. Even when optimized, the LOFO process causes metal films contacted to the SAM to be torn and wrinkled (peak to peak roughness $=5 \mathrm{~nm}) .{ }^{[10 \mathrm{c}]}$ The additional polymer layer in the PALO process prevents wrinkling and tearing and allows for an array of electrodes to be transferred simultaneously, creating multiple devices in one step. ${ }^{[10 \mathrm{~d}]}$ The authors state that using the PALO method, $90 \%$ of non-shorting molecular junctions were created. However, these studies were carried out on thick langmuir-blodgett films, with no studies to our knowledge being carried out on SAMs. 
(a)

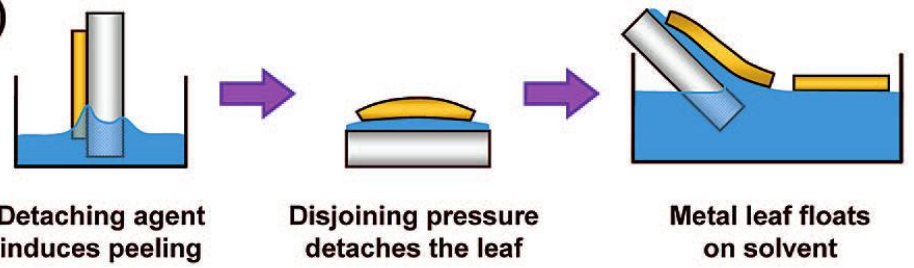

(b)

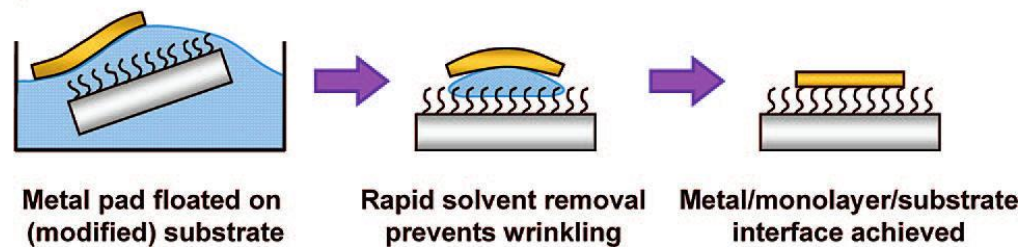

Figure 3.3: Schematic off the lift-off-float-on (LOFO) process; (a) lift-off of the metal film from a glass slide using a suitable solvent/detaching agent, (b) float-on of the metal film onto a substrate modified with a SAM in a clean solvent (Figures (a) and (b) reprinted with permission from. ${ }^{[15]}$ Copyright (C) 2008, American Chemical Society).

The conductive polymer technique conceived by de Boer and co-workers, ${ }^{[10 e]}$ has proven to be a very successful strategy in forming molecular tunneling junctions. The conductive polymer mix of poly(3,4-ethylenedioxythiophene) stabilized with poly(4-styrenesulphonic acid) (PEDOT:PSS) is spin-coated on top of the SAM, with a Au layer subsequently being vapor deposited on top, creating PEDOT:PSS tunneling junctions (Figure 3.4a). The PEDOT:PSS acts as a protective layer preventing the vapor deposited $\mathrm{Au}$ atoms from penetrating and damaging the SAM, whilst still promoting electrical conduction through the tunneling junction. This technique has been used to investigate the electrical characteristics of a variety of SAMs with yields of working junctions of $>90 \%$. Additionally, these junctions have been found to be stable for months at a time, with even some SAMs being investigated with multiple junctions connected in series. ${ }^{[16]}$ However, due to the successful use of this technique to fabricate tunneling junctions, its drawbacks have been extensively investigated and are as follows. 
a)

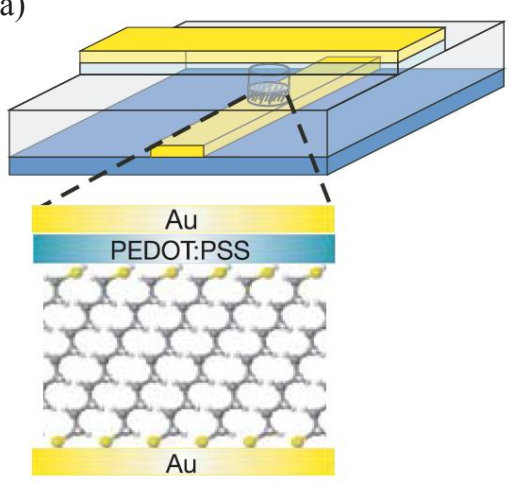

b)

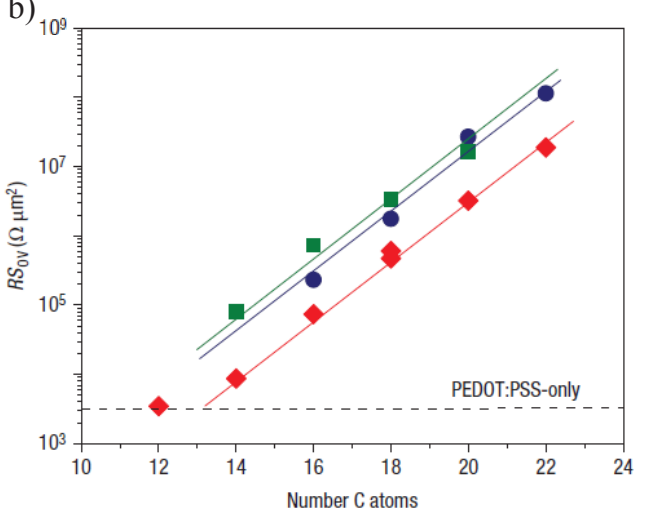

Figure 3.4: (a) Schematic representation of an alkanedithiol SAM immobilized within a PEDOT:PSS tunneling junction (Figure adapted by permission from Macmillan Publishers Ltd: Nature, ${ }^{[10 \mathrm{e}]}$ Copyright (C) 2006). (b) A semi-log plot of the normalized resistance $(R S)$ as a function of the number of carbon atoms for SAMs of alkanethiols, $\mathrm{C}_{\mathrm{n}} \mathrm{H}_{2 \mathrm{n}+1} \mathrm{SH}\left(\mathrm{C}_{\mathrm{n}} \mathrm{SH}\right)(\mathrm{n}=14-22)$, immobilized within PEDOT:PSS tunneling junctions that have been prepared using different process parameters. Red diamonds $=$ junctions made with the default process flowchart using L6000.5 photoresist and Agfa ICP new-type PEDOT:PSS with 5\% DMSO. Blue circles $=$ junctions made using PH500 PEDOT:PSS with 5\% DMSO. Green squares $=$ junctions made using the MA1407 photoresist instead of L6000.5. Dotted line = the PEDOT:PSS-only value. (Figure reprinted by permission from Macmillan Publishers Ltd: Nature Nanotechnology, ${ }^{[17]}$ Copyright $\odot 2008$ ).

The fabrication of PEDOT:PSS junctions require expensive clean room procedures such as photolithography. Also, small variations in the fabrication procedure, such as using different commercially available PEDOT:PSS formulations and different types of photoresist can cause a variation in the absolute current measured (Figure 3.4b) and using $\mathrm{SiO}_{2}$ instead of photoresist decreases the overall yield of working devices (from $>90 \%$ to $\sim 58 \%$ ). ${ }^{[18]}$ Additionally, upon spin-coating the PEDOT:PSS layer on top of the SAM, it may engulf the SAM. This could be an explanation for the higher $J$ values and lower $\beta$ values obtained in PEDOT:PSS tunneling junctions ${ }^{[10 \mathrm{e}]}$ compared to other techniques, and could also inhibit the true functional characteristics of the SAM investigated.

PEDOT:PSS itself is hydroscopic and hence contains water in ambient conditions, with the electrolysis of water leading to a small amount of hysteresis in the $J(V)$ measurements at lower voltages $(<1.0 \mathrm{~V})$. At higher voltages $(>2.0 \mathrm{~V})$ it leads to the formation of $\mathrm{H}_{2}$ gas inside the enclosed junction, causing it to blow up. ${ }^{[19]}$ As PEDOT:PSS is organic, it does not 
exhibit the same electrical characteristics as the traditional metal electrode, thus may influence the $J(V)$ characteristics of the SAM, for example during temperature dependent charge transport investigations. ${ }^{[20]}$ Finally, PEDOT:PSS films are comprised of spherical grains of 20 to $50 \mathrm{~nm}$ with PEDOT rich cores surrounded by PSS rich shells. This causes an inhomogeneous PEDOT:PSS layer for junctions of $<5 \mu \mathrm{m}$ in diameter, leading to a larger variation of $J$ values measured.

The recently developed 'Graphene Electrode' technique allows fabrication of molecular tunneling junctions by using two similar methods. ${ }^{[10 \mathrm{i}, 10 \mathrm{j}]}$ In the initial study a multilayer graphene film (mGF) $\left(<10 \mathrm{~nm}\right.$ thick) was transferred on top of the $\mathrm{SAM},{ }^{[10 \mathrm{i}]}$ whilst in the most recent study reduced graphene oxide (rGO) was dissolved in DMF, with the supernatant of the rGO solution used to spin coat a rGO film $(\sim 10 \mathrm{~nm}$ thick $)$ on top of the SAM (Figure 3.5). ${ }^{[10 j]}$ In both cases an Au layer was vapor deposited on top of the graphene based layer. In the same manner as PEDOT:PSS, graphene acts as a conducting protective layer, preventing the vapor deposited $\mathrm{Au}$ atoms from penetrating and damaging the SAM, whilst still allowing electron transport through the tunneling junction. Both techniques have shown the ability to collect statistically relevant numbers of data, with yields of working junctions of $\sim 90 \%$ for $\mathrm{mGF}$ tunneling junctions and $>99 \%$ for rGO tunneling junctions. Also, both techniques have been found to produce molecular tunneling junctions that are stable for prolonged periods of time, with the original $J(V)$ characteristics being reproduced for mGF tunneling junctions after storage in ambient conditions for 40 days and for rGO tunneling junctions after storage under vacuum for 30 days. Additionally, the mGF tunneling junctions have shown good thermal stability (better than that of PEDOT:PSS), allowing temperature dependent measurement to be performed.

Due to the recent nature of graphene electrode techniques, their (dis)advantages are still not well understood, yet it is clear that the fabrication of the bottom electrodes in the mGF tunneling junctions require expensive photolithography techniques limiting the usage to well-equipped laboratories and institutions. ${ }^{[10 \mathrm{i}]}$ A more speculative disadvantage in the $\mathrm{mFG}$ tunneling junctions is that the process in which the solid multi-layer graphene film is transferred to the substrate as the top electrode seems cumbersome, leading to an ill-defined interlayer. The fabrication process has been simplified in rGO tunneling junctions with the spin-coating from DMF of the rGO top electrode. ${ }^{[10 \mathrm{j}]}$ However, the homogeneity of this layer is ill-defined as the rGO sheets were found to overlap each other. Additionally, as 
DMF has a high boiling point, the complete removal of the solvent must be difficult, or just simply impossible. Finally, for an unknown reason all $J(V)$ measurements for the rGO tunneling junctions were performed under vacuum.

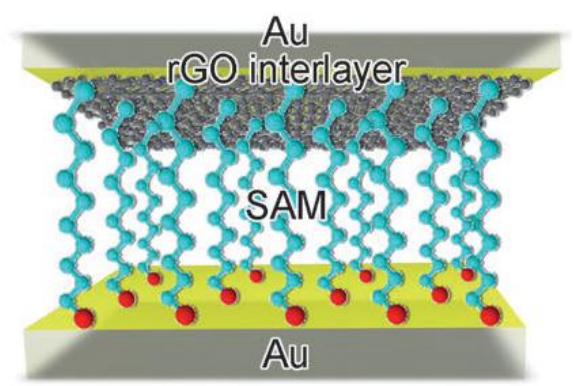

Figure 3.5: Schematic representation of a alkanethiol SAM immobilized within a rGO molecular tunneling junction (Figure reprinted with permission from. ${ }^{[10 j]}$ Copyright $(C)$ 2011, John Wiley and Sons).

The hanging mercury $(\mathrm{Hg})$ drop tunneling junction is a relatively cheap and simple technique to construct in general lab space, which overcomes the excessive clean room expense required for some other techniques. It consists of a drop of $\mathrm{Hg}$ protruding from a metal syringe, covered with a protective alkanethiol SAM (Figure 3.6). ${ }^{[10 \mathrm{f}, 10 \mathrm{~g}, 11,21]} \mathrm{The} \mathrm{Hg}$ drop is then lowered into contact with a second SAM (SAM of interest) formed on a second (bottom) $\mathrm{Hg}$ drop (Figure 3.6a) ${ }^{[10 \mathrm{f}, 11 \mathrm{~b}, 21 \mathrm{~b}]}$ or a solid metal film ${ }^{[10 \mathrm{~g}, 11]}$ (Figure 3.6b), in a solution of the protective alkanethiol. Advantageously, $\mathrm{Hg}$ can form well-ordered SAMs in seconds and self-correct defects such as pinholes present in a SAM. Additionally, the liquid $\mathrm{Hg}$ surface is smooth and does not have structural features such as step edges, grains, pits and lattice structures, which are present in solid metal films and cause defects to occur in the absorbed SAM. Finally, the Hg drop makes good conformal contact with SAMs and conforms readily to the topography of solid surfaces.

However, this technique has several drawbacks. ${ }^{[10 \mathrm{f}, 10 \mathrm{~g}, 11,21]} \mathrm{Hg}$ itself is volatile, toxic, and easily amalgamates with metals such as $\mathrm{Au}, \mathrm{Ag}$ and $\mathrm{Cu}$. To stabilize the junctions, minimize amalgamation and optimize reproducibility, the $J(V)$ measurements are carried out in an alkanethiol solution. The alkanethiol forms a protective SAM layer on the Hg drop, creating an additional tunnel barrier, which along with the liquid itself provides unwanted additional parameters. Under an applied potential, $\mathrm{Hg}$ suffers from electronmigration, which changes the shape of the material, causing a lateral movement of the SAMs on the $\mathrm{Hg}$ electrode, and the junctions are only stable for $100-200$ seconds/scans. For these reasons the Whitesides 
research group abandoned the use of this technique in their charge transport/molecular tunneling junction studies, opting to use the similar but slightly different material of Eutectic Gallium Indium (EGaIn). Nevertheless, there are various other groups still using the $\mathrm{Hg}$ drop in their charge transport investigations. ${ }^{[22]}$

a)

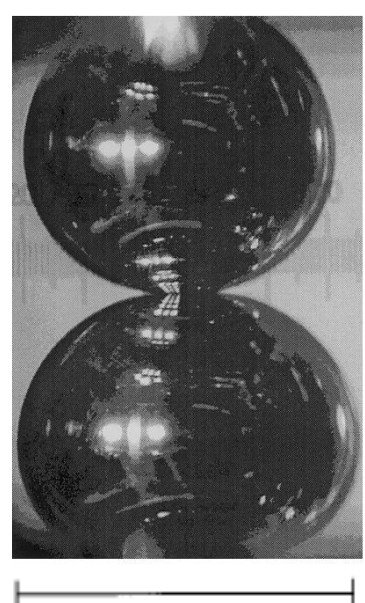

b)

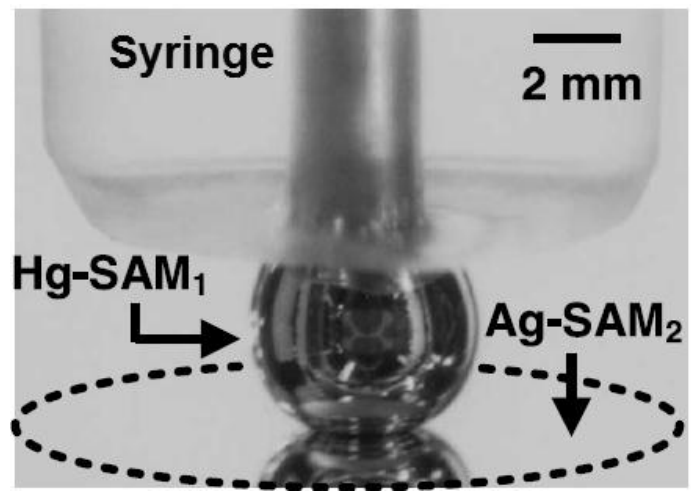

$1 \mathrm{~mm}$

Figure 3.6: Photographic images of hanging $\mathrm{Hg}$ drop tunneling junctions; (a) where both the top and bottom electrode are a $\mathrm{Hg}$ drop covered with SAM, (b) where the $\mathrm{Hg}$ drop top electrode covered with a protective SAM is in contact with the SAM of interested immobilized on a Ag surface (Figure (a) reprinted from, ${ }^{[21 b]}$ Copyright (C) 2000, with permission from Elsevier, Figure (b) reprinted from, ${ }^{[21 a]}$ Copyright (C) 2002, with permission from Elsevier).

Eutectic Gallium Indium (EGaIn) has been used as an alternative to $\mathrm{Hg}$, with it being used as the top electrode in two different experimental setups. ${ }^{[2,23]}$ The first is a direct substitution of the hanging $\mathrm{Hg}$ drop junction, and referred to in this thesis as 'the EGaIn technique' ${ }^{[2]}$ As in the $\mathrm{Hg}$ drop junctions, a drop of EGaIn is suspended from a metal syringe. However, due to a layer of $\mathrm{Ga}_{2} \mathrm{O}_{3}$ that forms on the surface of the EGaIn, using a sacrificial surface, it can be shaped into metastable, nonspherical structures (such as conical probes) (Figure 3.7a), eliminating the need for a protective alkanethiol layer and a solvent bath. Therefore, EGaIn can make conformal non-damaging contact with the SAM in ambient conditions, producing working junctions with yields of $\sim 85 \%$, that are typically stable for hours at a time. ${ }^{[2-3]}$ In the second set-up, EGaIn electrodes are stabilized in PDMS channels to create an 'EGaIn device' (Figure 3.7b). ${ }^{[23]}$ This increases the stability of the junctions to 2-3 days, and since 
the $J(V)$ characteristics of EGaIn itself are temperature independent, allows for temperature dependent charge transport investigations. Finally, unlike Hg, EGaIn is non-toxic.

However, EGaIn's strongest attribute, the $\mathrm{Ga}_{2} \mathrm{O}_{3}$ layer, is also its greatest weakness, leading to several ambiguities within the junctions, which are openly acknowledged. ${ }^{[3,23-24]}$ Even after extensive investigations, the exact thickness and resistivity of the $\mathrm{Ga}_{2} \mathrm{O}_{3}$ layer is unknown. The exact nature of the interface between the SAM and $\mathrm{Ga}_{2} \mathrm{O}_{3}$ layer (the molecules investigated thus far are believed to form a van der Waals contact with $\mathrm{Ga}_{2} \mathrm{O}_{3}$ ) and also the real contact area is ill-defined, with the latter being due to the unknown surface roughness of $\mathrm{Ga}_{2} \mathrm{O}_{3}$. Also, the 'EGaIn technique' junctions are assembled in ambient conditions, and therefore the effect that physisorbed organic material on the surface of $\mathrm{Ga}_{2} \mathrm{O}_{3}$ has on the electrical characteristics is uncertain. Therefore, to overcome these uncertainties using this technique, it is essential to carry out the appropriate control experiments (as it is with all tunneling junction techniques). Additionally, although the 'EGaIn device' offers some added advantages over the 'EGaIn technique', the fabrication of the devices requires more time than an 'EGaIn technique' junction, and also requires expensive clean room procedures such as photolithography. Finally, even though both EGaIn experimental set-ups are more stable than the $\mathrm{Hg}$ drop technique, they are only adequate for physical-organic studies of charge transport through SAMs, and are not as stable as the PEDOT:PSS and Graphene techniques. 
a)

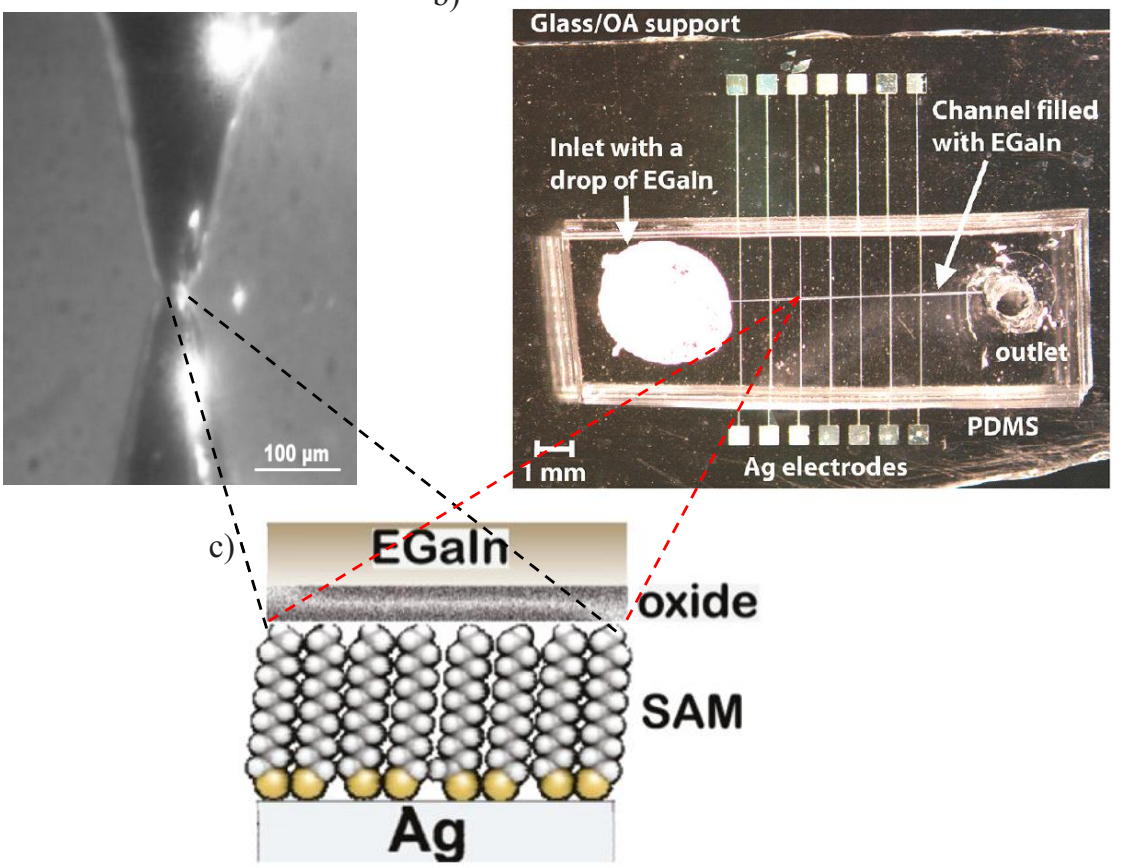

Figure 3.7: (a) Photographic image of the formation of an EGaIn tunneling junction, lowering of the EGaIn tip onto a SAM immobilized on a metal (Ag) film, with contact being indicated upon convergence of the EGaIn tip and its reflection on the metal (Ag) film. (b) Photographic image of the EGaIn device, with EGaIn being stabilized in PDMS channels across patterned Ag electrodes on glass. (c) Cross section of the SAM immobilized on the Ag film within the EGaIn tunneling junction/device. (Figures (a) and (c) reprinted with permission from. ${ }^{[25]}$ Copyright (C) 2011, American Chemical Society, Figure (b) reprinted with permission from. ${ }^{[23]}$ Copyright $(\subset) 2010$, American Chemical Society.

To directly investigate the technique's ability to form SAM tunneling junctions, it is best to firstly take into account the yield of working junctions (in optimum state), secondly the facilities required to fabricate them, thirdly the stability of the junctions, as this may affect the yield of working junctions, due to there not being a universal definition for a working junction (i.e. in some cases a working junction is defined as a junction that only needs to be able to perform five $J(V)$ scans, whereas others require twenty $J(V)$ scans). Finally, there is the type of investigation or application required, i.e., if temperature dependent measurements are required, if a specific SAM needs to be investigated and if the level of toxicity can be controlled (Table 3.1). 
Table 3.1: Comparison of Molecular Tunneling Junction Techniques*

\begin{tabular}{|c|c|c|c|c|c|c|c|}
\hline $\begin{array}{l}\text { Molecular Junction } \\
\text { Technique }\end{array}$ & $\begin{array}{l}\text { Yield of } \\
\text { working } \\
\text { junctions } \\
(\%)^{[b]}\end{array}$ & $\begin{array}{l}\text { Facilities } \\
\text { Needed } \\
(\mathrm{C}) /(\mathrm{L})^{[\mathrm{c}]}\end{array}$ & $\begin{array}{l}\text { Time of } \\
\text { Stability } \\
\text { [d] }\end{array}$ & $\begin{array}{l}\text { Time of } \\
\text { Fabrication }\end{array}$ & Toxic & $\begin{array}{l}\text { Temperature } \\
\text { Independent }^{[t]}\end{array}$ & $\begin{array}{l}\text { SAM } \\
\text { Limitation }\end{array}$ \\
\hline 1 metal evaporated & $<5$ & $\mathrm{C} / \mathrm{L}$ & NA & Hours & No & Yes & No \\
\hline $2 \mathrm{nTP}$ & $<20$ & $\mathrm{C}$ & NA & Days & No & NA & Yes \\
\hline 3 LOFO & $\mathrm{NA}$ & $\mathrm{C} / \mathrm{L}$ & NA & Days & No & NA & Yes \\
\hline $4 \quad$ PALO & $>90 / \mathrm{NA}$ & $\mathrm{C}$ & NA & Days & No & NA & Yes \\
\hline 5 PEDOT:PSS & $>90(\sim 58)$ & $\mathrm{C}$ & Months & Hours & No & Yes\# & Yes \\
\hline $6 \mathrm{mGF}$ & -90 & $\mathrm{C}$ & Months & Hours & No & Yes & No \\
\hline $7 \mathrm{rGO}$ & $>99$ & $\mathrm{C} / \mathrm{L}$ & Months & Hours/Days & No & NA & No \\
\hline $8 \mathrm{Hg}(1)$ & - & $\mathrm{L}$ & Seconds & Minutes & Yes & NA & No \\
\hline $9 \quad \mathrm{Hg}(2)$ & $33-81$ & $\mathrm{~L}$ & Seconds & Minutes & Yes & NA & No \\
\hline 10 EGaIn technique & $\sim 85$ & $\mathrm{~L}$ & Hours & Minutes & No & No & No \\
\hline 11 EGaIn device & $70-90$ & $\mathrm{C}$ & Days & Hours/Days & No & Yes & No \\
\hline
\end{tabular}

[a]; 1 = metal evaporated directly onto the SAM, 2 = nano-transfer printing, $3=$ lift-off-float-on, $4=$ polymerassisted lift-off (PALO), $5=$ the conductive polymer mix of poly(3,4-ethylenedioxythiophene) stabilized with poly(4-styrenesulphonic acid), $6=$ multilayer graphene film, $7=$ reduced graphene oxide, $8=$ both the bottom and top electrode are drops of $\mathrm{Hg}, 9=$ bottom electrode is a solid metal surface and top electrode is $\mathrm{Hg}$ drop, $10=$ EGaIn drop technique, $11=$ EGaIn device. $[\mathrm{b}]=$ based on studies performed on alkanethiol SAMs. [c]; $(\mathrm{C})=$ clean room facilities, $(\mathrm{L})=$ general lab space. [d] = length of time $J(V)$ measurements are reproducible. $[\mathrm{e}]=$ Time required to fabricate the top electrode. $[\mathrm{f}]=$ does not greatly influence charge transport characteristics over the temperature range of $250-300 \mathrm{~K}$, allowing for charge transport investigations of SAMs at various temperatures, \# a study carried out by Lee and co-workers ${ }^{[10 \mathrm{i}]}$ shows that at temperatures $>300 \mathrm{~K}$, the $J(V)$ characteristics are less influenced in mGF tunneling junctions than in PEDOT:PSS tunneling junctions. [g], suffers from needing specific SAM metal interaction, or can hinder SAM functionality.

*All data presented must fall within the scope of the discussion (i.e., the molecular tunneling junction must consist of a SAM, and must be $>1 \mu \mathrm{m}$ in diameter)

$\mathrm{NA}=$ Not available - defined as no known information has been published on the criteria, within the scope of this discussion (i.e., $\mathrm{Hg}$ has been used to perform temperature dependent measurements across molecular junctions, however the molecular layer was not a $\mathrm{SAM}^{[26]}$ )

The EGaIn technique was the most appropriate technique to build at the University of Twente to carry out fundamental physical-organic charge transport studies. In the following section (3.2) of this chapter the construction of the EGaIn technique at the University of Twente is described. 


\subsection{Construction of the EGaIn technique}

As only minimal amounts of specialized equipment are needed and a clean room environment to evaporate metals, or perform lithographic patterning (which is used for the fabrication of some of other molecular tunneling junctions) is not necessary, this technique can be easily accessible to a wide range of laboratories. Explained below are the materials and procedure used to re-construct the EGaIn technique in our laboratory.

\subsubsection{Materials and Construction}

Figure 3.8 shows photographic images of the EGaIn technique and Table 3.1 in the experimental details (section 3.5) displays the components required to build each apparatus in the EGaIn technique. As can be seen from these images (and Table 3.1 in the experimental details), the only expensive piece of equipment is the Keithley 6430 sub-femtoamp remote source meter. Also in the images it can be seen that the technique sits on top of concrete stones ${ }^{[27]}$ in an ambient environment. 


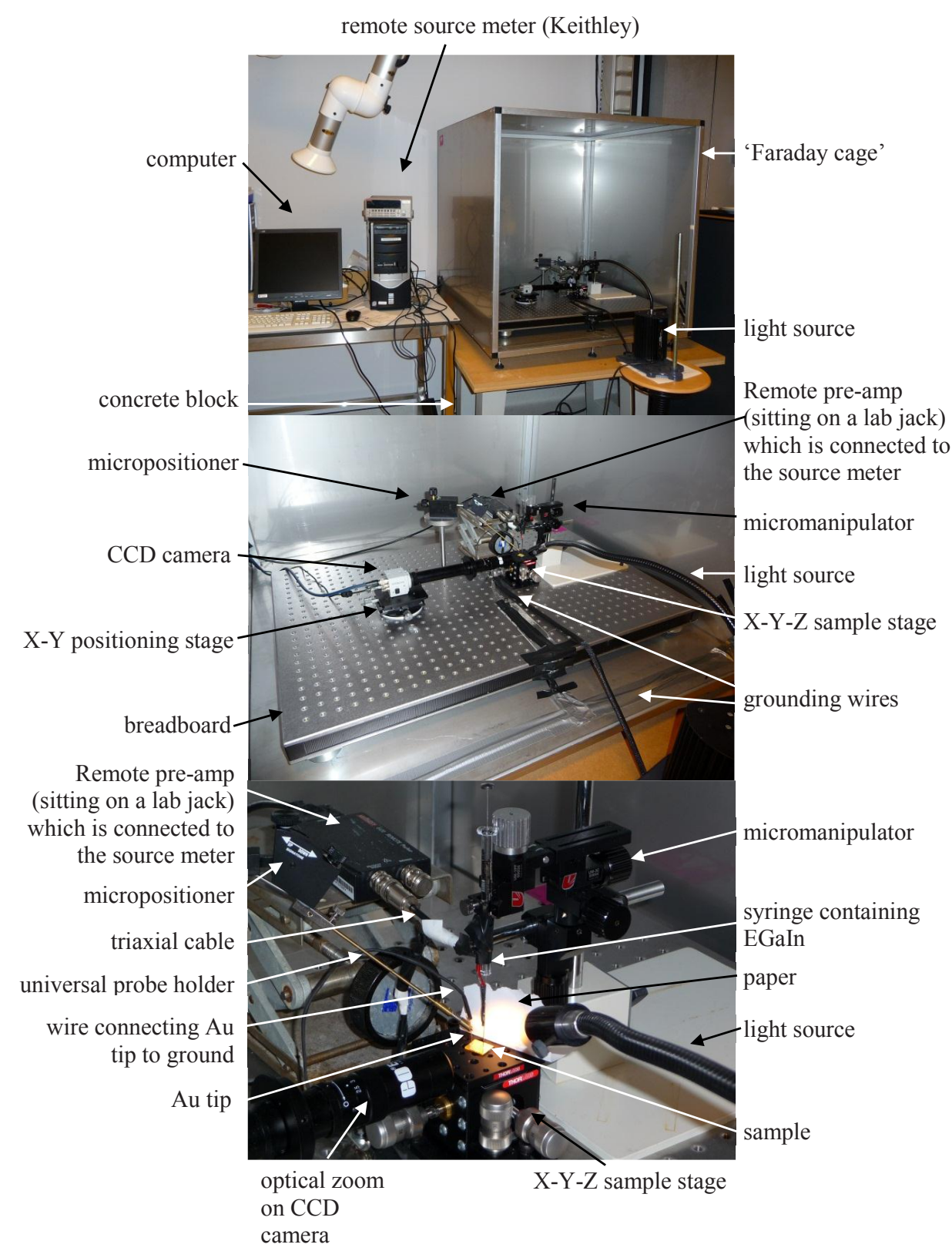

Figure 3.8: Photographic Images of the EGaIn technique built at the University of Twente; overview of whole apparatus (top image), overview of components attached to the breadboard (middle image), zoomed in overview of components, and example of junction formation (bottom image). 
General Framework: The breadboard where each apparatus was to be mounted was placed inside a large metal 'Faraday cage', which, as it is not closed, is mainly used to protect the apparatus from the external environment, air drafts, dust etc. (Figure 3.8 top and middle). The following apparatus are mounted onto the breadboard as described below.

CCD Camera: The X-Y positioning stage was mounted towards the front left hand side of the breadboard. Using a 'home made' bracket, the CCD camera which has an optical lens attached, was mounted to the X-Y positioning stage (Figure 3.8 middle). This allows the CCD camera to be moved independently in the $\mathrm{X}-\mathrm{Y}$ direction. Optimally an $\mathrm{X}-\mathrm{Y}-\mathrm{Z}$ positioning stage would give greater versatility, however an X-Y stage is sufficient.

X-Y-Z Sample Stage: A breadboard adapter plate was attached to the microblock X-Y-Z sample stage, which in turn was mounted towards the front center of the breadboard, in line with the CCD camera (Figure 3.8 middle and bottom).

Micropositioner: A steel post was screwed into the breadboard, slightly behind and in between the CCD camera and sample stage. A 'homemade' metal disc was screwed on top of the steel post to act as a platform for the micropositioner (Figure 3.8 middle). The Universal Probeholder was attached to the Micropositioner, with the Micropositioner then being attached to the metal disc using its magnetic base. A Au coated probe was placed into the Universal Probeholder to act as the counter probe, which is grounded (Figure 3.8 bottom). As the micropositioner is placed on the left hand side in this setup, a left handed micropositioner is used, however, if an inverted version of this setup would be built, a right handed micropositioner should be used.

Micromanipulator: Extra mounting holes were drilled into the iron plate which was then screwed onto the breadboard on the right hand side, in close proximity of the sample stage. After assembled, the X-Y-Z-micromanipulator was magnetically attached to the iron plate in such a way that the 'holder' overhung the sample stage. The syringe that would contain the EGaIn was placed inside the holder (Figure 3.8 middle and bottom). 


\subsubsection{Wiring and Connecting to the Electrometer/Remote Source Meter (Keithley) and Computer}

A Y-C S-Video cable was used to connect the CCD camera to an external video grabber, which by using a USB cable, was connected to the computer, allowing the images from the camera to be seen on the computer screen. The Keithley 6430 sub-femtoamp Remote Source Meter (Keithley) was situated outside of the Faradaic Cage, on or next to the computer. The Keithley was connected to the computer using a GPIB connected from the mainframe (back) of the Keithley (Figure 3.9a) to a USB port in the back of the computer. To allow for low potentials to be applied and low currents to be measured, this Keithley has a remote preamplifier (remote preamp). The remote preamp was placed on a small jack, inside the Faraday Cage, on the breadboard, behind the sample stage, in between the micropositioner and micromanipulator (Figure 3.8 middle and bottom). The remote preamp was connected to the preamp connection on the rear panel of the Keithley mainframe by feeding a preamp cable through a small hole cut in the side of the Faraday cage. The remote preamp has two outputs, labeled in Figure 3.9b as IN/OUT HIGH and SENSE. As the IN/OUT HIGH is used to source-measure volts, amps and ohms and the SENSE is used for 4 wire sense measuring, the tri-axial cable was connected IN/OUT HIGH output and capped the SENSE output, as only two terminal $I(V)$ measurements will be performed. To optimize the sensitivity and eliminate external effects such as electric fields, the tri-axial cable was cut as short as possible, minimizing the distance between the remote preamp and the apparatuses that the tri-axial cable will be connected to (the syringe containing the EGaIn attached to the micromanipulator, and the counter/ground probe attached to the micropositioner), whilst still allowing enough length on the cable to minimize tension on the wire which will lead to unstable junctions and cause damage to the wire itself. Figure 3.9c shows a cross section of the three different wire components that make up the tri-axial cable. When connected to the IN/OUT HIGH output, the three cables are as follows, the most inner is the HI (Input/Output High), the middle is the GUARD (Cable Guard) and the most outer is the LO (Input/Output Low), which is also known as the ground. When shortening the tri-axial cable the 3 wires were stripped back and separated. The HI was soldered directly to the needle of the EGaIn syringe, the GUARD was bypassed as when it was connected $J(V)$ displayed a recurring switching effect, and the LO/Ground was attached to the physical ground formed by the ground wire attached to the breadboard. An additional wire was soldered to the back end of the grounded $\mathrm{Au}$ counter probe that was in the universal probe holder attached to the 
micropositioner. This wire was also connected to the ground on the breadboard. The breadboard ground point was also used as the point where the Faradaic Cage is grounded to.

The grounding point was achieved by attaching a copper braided wire to a bracket, which was then screwed onto the breadboard. The other end of the copper wire was also coupled to a bracket and was attached to a grounding point within the room. The grounding wire of the power board, where all of the electrical apparatuses were plugged in, was taken out of the power board and attached to the same grounding point within the room, and therefore was not attached to the power plug in the wall. This gave a common ground for all apparatus in the set up. 

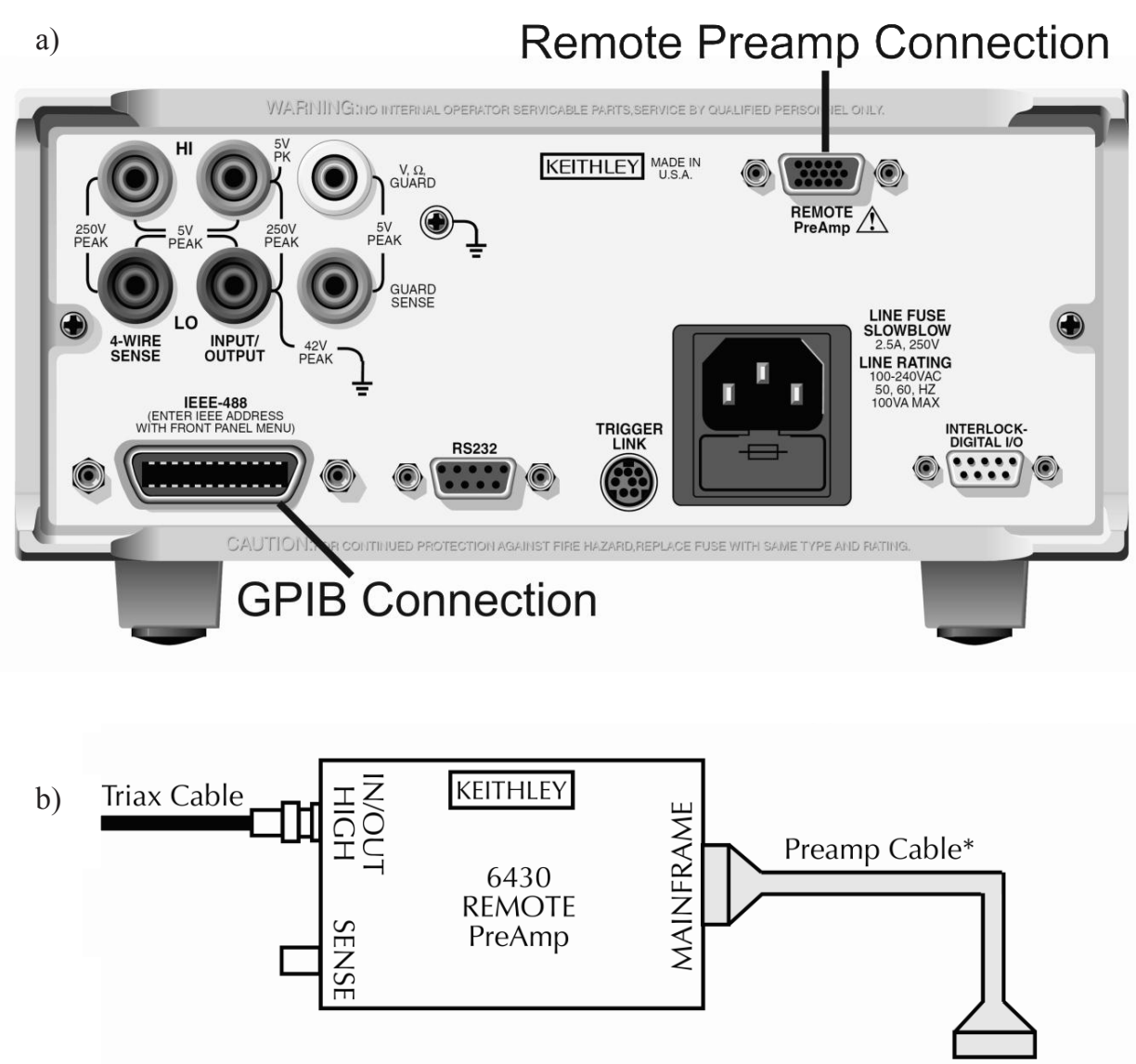

Connect to REMOTE PreAmp connector on rear panel of mainframe

c)

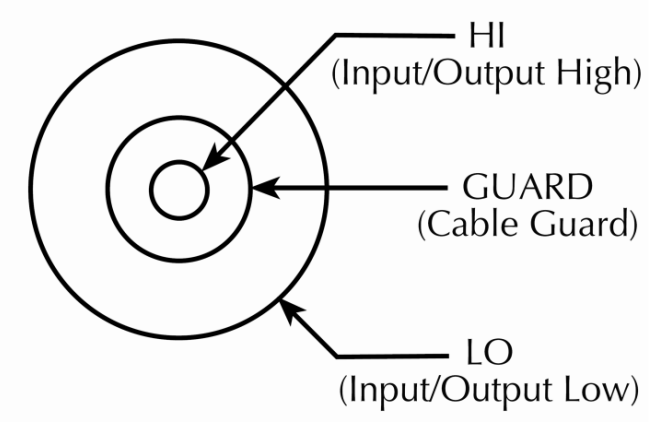

Figure 3.9: Mainframe (back side) of the Keithley (a), remote preamp (b), cross-section of triaxial cable (c) (Figures (a - c) adapted with permission from. ${ }^{[28]}$ Copyright $\odot$ 2008, Keithley Instruments, Inc.). 


\subsubsection{Resistor Tests}

Once constructed the EGaIn technique was tested for its accuracy and sensitivity when performing $I(V)$ measurements by using standard metal film resistors. The experimental limit for this technique can be seen from the $I(V)$ measurements performed $\pm 5 \times 10^{-4} \mathrm{~V}$ $(0.5 \mathrm{mV})$ across a $10 \mathrm{M} \Omega$ resistor, as it did not give an ohmic response (Figure 3.10). The $I(V)$ shows a small amount of hysteresis at $0 \mathrm{~V}$, with a maximum offset of $\sim+5 \mathrm{pA}$ and the measured resistance was $10.5 \mathrm{M} \Omega$ at $+0.5 \mathrm{mV}$ and $11.6 \mathrm{M} \Omega$ at $-0.5 \mathrm{mV}$. Therefore, when performing a voltage sweep, the EGaIn technique has an offset of $\sim+5 \mathrm{pA}$. However, when performing $I(V)$ measurements across the supramolecular tunneling junctions the minimum bias range investigated is $\pm 2.0 \mathrm{~V}$, with $I$ being measured every $0.1 \mathrm{~V}$. Also as a majority of the data analyzed is based on $I$ measured at $\pm 2.0 \mathrm{~V}$, with the values of $I$ obtained being in the order of 10's to 100's of nA, an offset of $\sim+5 \mathrm{pA}$ is negligible and more than acceptable for the use of this technique.

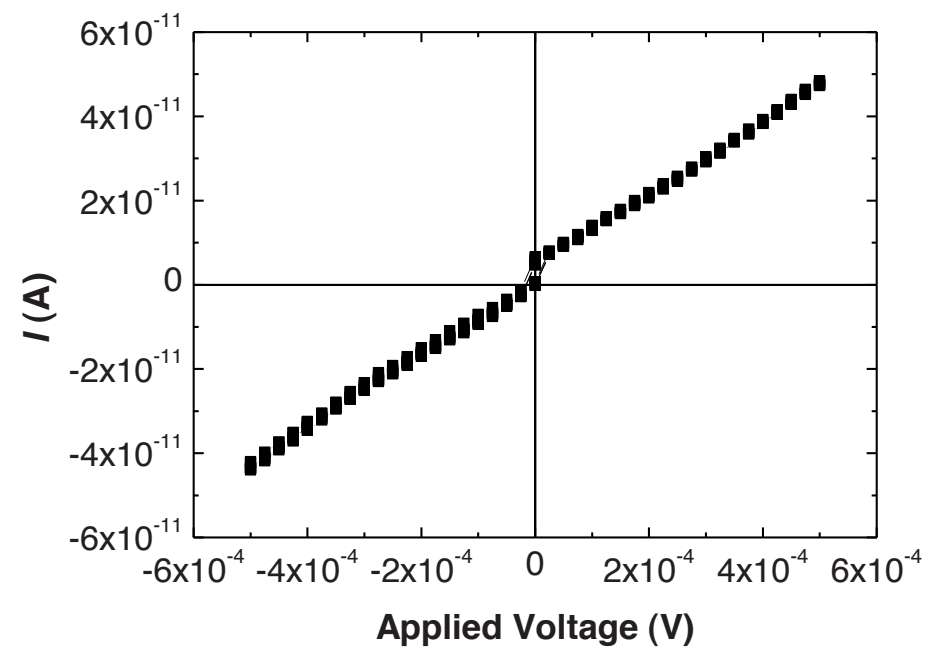

Figure 3.10: Three $I(V)$ scans performed $+/-0.0005 \mathrm{~V}(0.5 \mathrm{mV})$ across a $10 \mathrm{M} \Omega$ resistor using the EGaIn drop technique.

\subsubsection{Challenges with the Setup}

Very occasionally (if occurs, then usually only during the first couple of scans), during the $J(V) / I(V)$ measurement, a small 'noise peak' is present approximately a quarter of the way during the positive and negative voltage sweep. Below this is shown when performing $I(V)$ measurements $\pm 5 \mathrm{mV}$ across a $10 \mathrm{M} \Omega$ resistor, with the 'noise peak' being present at \pm 
1.25/1.5 $\mathrm{mV}$ (Figure 3.11). This can be attributed to the Keithley source meter, as when the measured current changes from one magnitude to another, a 'noise peak' is present due to a delay in the Keithley averaging the data. As this phenomenon does not regularly occur during $J(V)$ measurements, it does not affect the averaged $J(V)$ data.

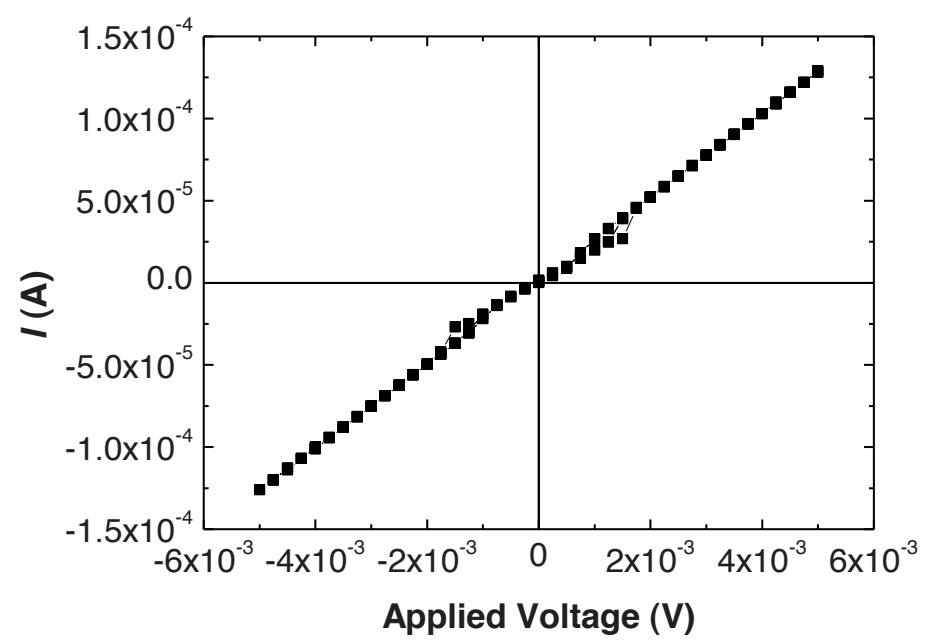

Figure 3.11: Three $I(V)$ scans performed $\pm 5.0 \mathrm{mV}$ across a $10 \mathrm{M} \Omega$ resistor using the EGaIn drop technique. A noise peak is present at $\pm 1.25 / 1.5 \mathrm{mV}$, where the measured current is changing magnitude, causing a delay in the Keithley averaging the data.

The limiting factor for the measurement sensitivity is the interface between the HI (input/output High) wire soldered to the syringe. Multiple attempts may be needed to obtain a good contact, and maximize sensitivity. It was found that attaching the wire lengthways along the syringe gave the best results. However, due to the materials used to solder the HI wire to the syringe, and applying continuous biases across the system, over time the wire starts to corrode, reducing the sensitivity of the setup. Typically, this does not reduce the sensitivity of the set up significantly over a period of months. However, in one particular measurement performed on a supramolecular tunneling junction, for an unknown reason (most likely a chemical reaction causing the wire-syringe contact to become oxidized, causing an increase in contact resistance), a very large reduction in sensitivity was apparent within $20 J(V)$ scans (Figure 3.12). In this measurement, as the scan number increases, the value of $|J|$ measured at $0 \mathrm{~V}$ increases, and a larger applied bias is required for $|J|$ measured to change sign/polarity ( from $\pm 0.5 \mathrm{~V}$ to $\pm 1.0 \mathrm{~V}$ ). Additionally, the same problem can occur for the grounded wire soldered to the Au probe in the universal probe holder. Therefore, 
regular tests with a resistor must be carried out in order to ensure that the system is working optimally. Other methods to attach the wires to the syringe could possibly entail using small alligator clips, however the pressure applied to the syringe by the clip, may destroy and destabilize the syringe.

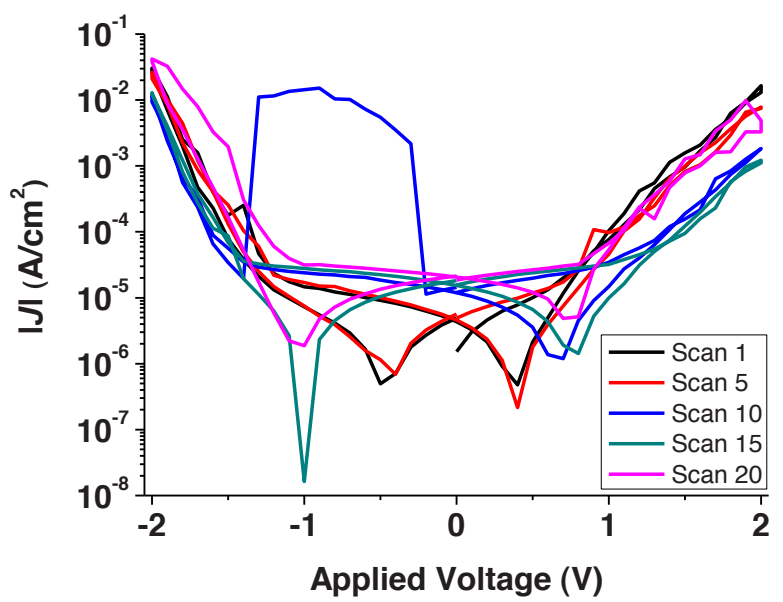

Figure 3.12: Semi-log plot of $J(V)$ measurements performed $\pm 2.0 \mathrm{~V}$ across a supramolecular tunneling junctions. The increase in the value of $|J|$ measured at $0 \mathrm{~V}$, and the larger the applied bias required for $\mid J$ measured to change sign/polarity (from $\pm 0.5 \mathrm{~V}$ to $\pm 1.0 \mathrm{~V}$ ) demonstrates the increase in the contact resistance between the HI (input/output High) wire and the EGaIn syringe.

The micromanipulator purchased to hold and position the syringe containing EGaIn, was not optimal. The knobs used to lower and retract the syringe are placed right next to and directly attached to the same extension the encompasses the EGaIn syringe (Figure 3.8 bottom) Therefore, upon adjusting these knobs to lower the EGaIn probe onto the surface, sometimes pressure from the hands move the EGaIn probe laterally and vertically, making it difficult to approach the SAM carefully, and at times affecting the yield of working devices by scratching the surface. To overcome this inconvenience, the EGaIn probe had to be placed in close proximity to the sample stage, with the sample stage then being carefully raised to bring the SAM into contact with the EGaIn probe. Whilst raising the stage, great care had to be taken in order to prevent the Au counter electrode, connected to the Au surface, moving and creating further complications upon forming the molecular junction. Nevertheless, once the EGaIn probe was in contact with the SAM, the micromanipulator was stable enough to regularly allow $J(V)$ measurements to be performed for $\sim 2$ hours and at times up to 16 hours 
(see Chapter 6). This complication of lowering the EGaIn probe onto the SAM can be easily solved in two different ways. Firstly, a more stable micromanipulator could be purchased, such as that sold by Leica and used in the EGaIn setup of Whitesides and co-workers. Secondly, the grounded counter electrode can be incorporated into a sample holder on the sample stage. Therefore, once the sample is secured in the holder, it is connected to ground, with the sample stage being able to move freely up and down in a stable manner and allowing the EGaIn syringe to be placed in a solid immovable support. The EGaIn setup built by Chiechi and co-workers at the University of Groningen is based around this idea.

The electrical characteristics of the $I(V)$ or $J(V)$ measurements of supramolecular tunneling junctions, at low biases (of approximately $\pm 0.6 \mathrm{~V}$ ) can be influenced by the contact area of the $\left(\mathrm{Ga}_{2} \mathrm{O}_{3}\right)$ EGaIn tip with the supramolecular layer. Contact areas of $\sim<100 \mathrm{~mm}^{2}$ commonly showed hysteresis in the voltage at $\sim+0.6 \mathrm{~V}$ (Figure 3.13a), whereas contact areas $\sim>100 \mu \mathrm{m}^{2}$ displayed an even hysteresis with the current changing at approximately opposing biases (Figure 3.13c) (which is the common trend for the $J(V)$ measurements performed in this thesis). The origin of this phenomena is unclear, but it is speculated that the small $\left(\mathrm{Ga}_{2} \mathrm{O}_{3}\right)$ EGaIn tips are dominated by the outer $\mathrm{Ga}_{2} \mathrm{O}_{3}$ layer and therefore this may affect $I$ and hence $J$ measured at lower biases. However, the values of $J$ measured at these biases are extremely small (typically $\mathrm{pA}$ ) and therefore, are only visible as $J$ is plotted on a $\log$ scale. If $J$ is plotted on a linear scale (Figure 3.13b and c) (as many others in the field do) these offsets are not visible. We choose to plot out our data on a log scale to be able to gather as much information as possible. 

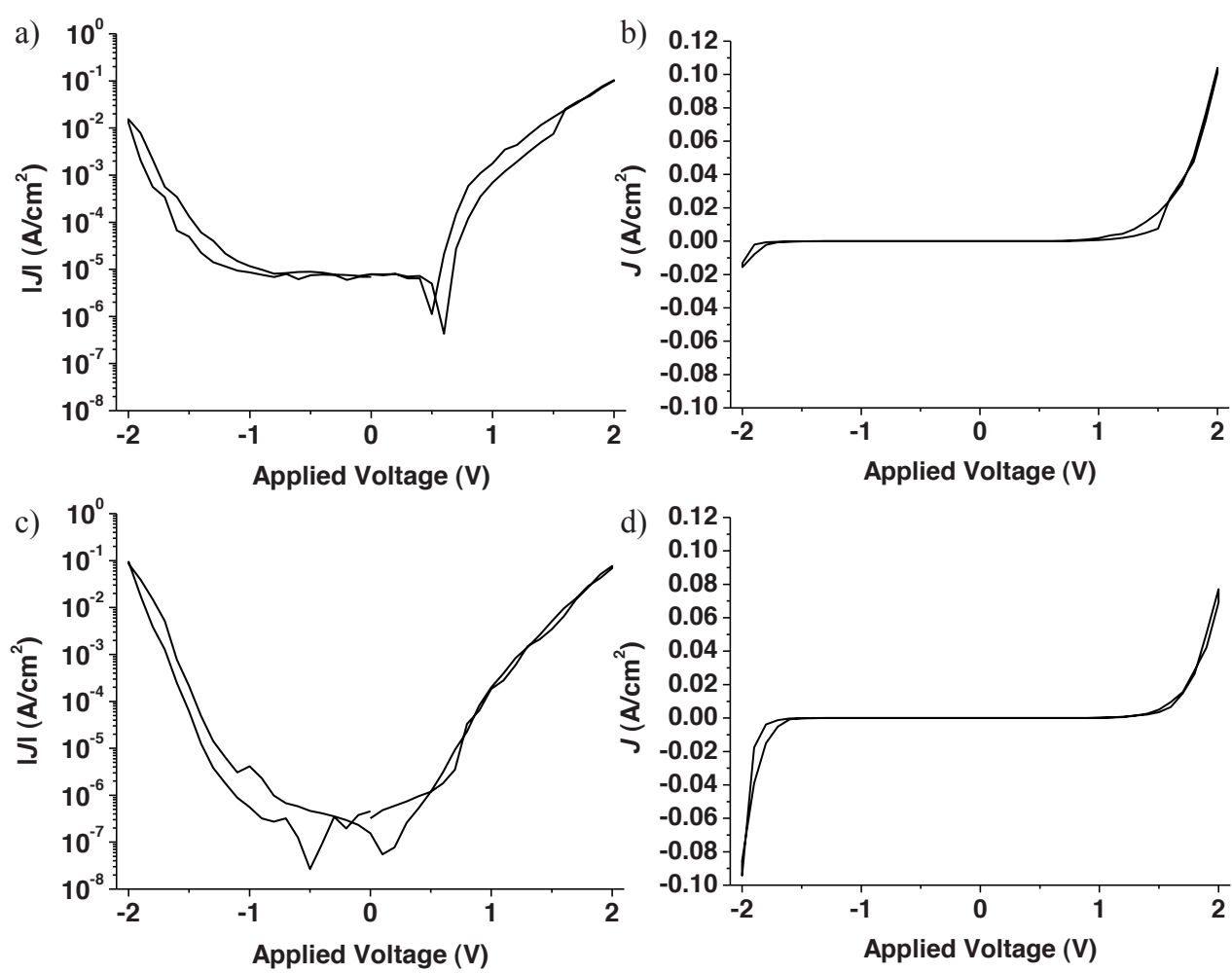

d) 0.12

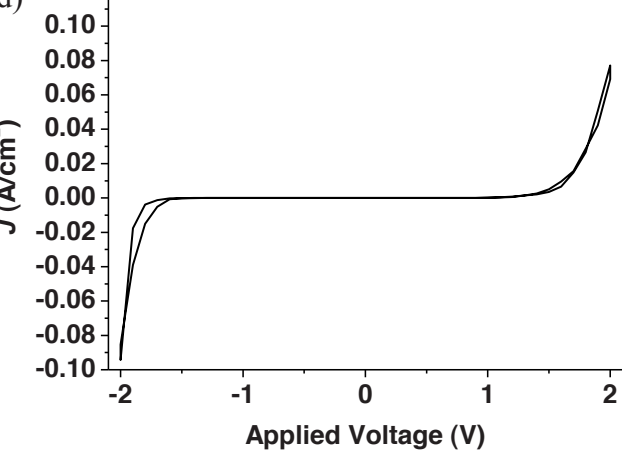

Figure 3.13: Semi-log plots of $J(V)$ measurements performed $\pm 2.0 \mathrm{~V}$ across supramolecular tunneling junctions, for contact areas of the $\left(\mathrm{Ga}_{2} \mathrm{O}_{3}\right)$ EGaIn tip with the supramolecular layer of $<\sim 100 \mu \mathrm{m}^{2}$ (a) and $>\sim 100 \mu \mathrm{m}^{2}$ (c). Linear plots of the same $J(V)$ measurements performed $\pm 2.0 \mathrm{~V}$ across supramolecular tunneling junctions, for contact areas of the $\left(\mathrm{Ga}_{2} \mathrm{O}_{3}\right) \mathrm{EGaIn}$ tip with the supramolecular layer of $<\sim 100 \mu \mathrm{m}^{2}$ (b) and $>\sim 100 \mu \mathrm{m}^{2}(\mathrm{~d})$. 


\subsection{Results and Discussion}

Described in this section is how to perform measurements on molecular tunneling junctions using the EGaIn technique, and how to statistically analyze the data obtained. Why it is important to accumulate statistically relevant numbers of data, how to minimize the amount of defects present in molecular tunneling junctions and how rectification can be used to investigate the $J(V)$ characteristics of molecular tunneling junctions is also discussed. Finally, the ability of the EGaIn technique to give reproducible data in different laboratories is shown by presenting the data obtained at the University of Twente for three supramolecular tunneling junctions that were also investigated in the laboratories of Whitesides and co-workers in the U.S.A.

\subsubsection{Performing Measurements on Molecular Tunneling Junctions}

As previously published by Whitesides and co-workers, ${ }^{[2]}$ to create an EGaIn drop junction an EGaIn probe must be formed. EGaIn probes are formed by suspending a drop of EGaIn from a metal $26 \mathrm{~s}$-gauge needle affixed to a $10-\mu \mathrm{L}$ syringe, bringing the drop into contact with the bare surface of a sacrificial metal film (usually Au or Ag on glass) placed on the sample stage, and using a micromanipulator and/or sample stage to retract the needle and/or sample stage slowly (ca. $50 \mu \mathrm{m} \mathrm{s}^{-1}$ ). The EGaIn adheres to both the needle and the metal film, allowing the drop of EGaIn to be pinched into an hour-glass shape until it bifurcates into two structures (Figure 3.14), one attached to the syringe (a cone approximately $0.05 \mathrm{~mL}$ in volume) and one (which was discarded) attached to the Ag. Conical tips of EGaIn can be produced with diameters ranging from less than $1 \mu \mathrm{m}$ to $100 \mu \mathrm{m}$; the larger the bore of the needle, and the more rapidly the syringe is raised, the larger the tip. The EGaIn tip protruding from the needle does not retract into a semispherical droplet (as would $\mathrm{Hg}$ ) due to the layer of $\mathrm{Ga}_{2} \mathrm{O}_{3}$ forming on the EGaIn's surface immediately upon contact with atmospheric oxygen. 


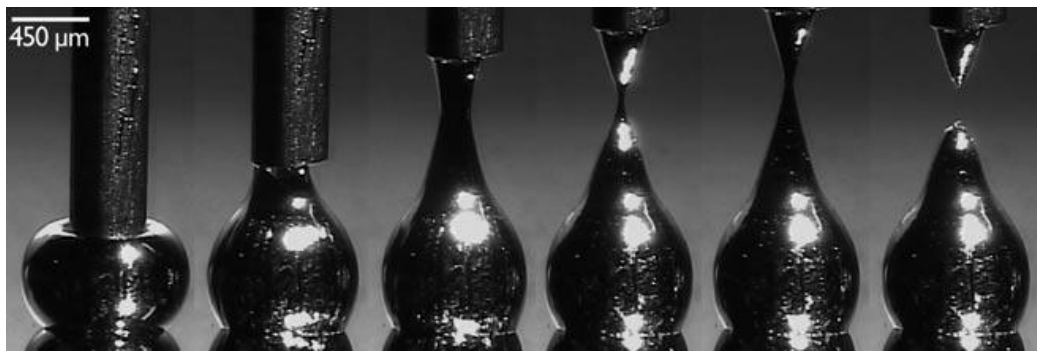

Figure 3.14: Image of the step by step formation of an EGaIn tip (Figure reprinted with permission from. ${ }^{\left[{ }^{2]}\right.}$ Copyright (C) 2007, John Wiley and Sons).

The EGaIn junction is created by placing the metal film covered with the SAM of interest onto the sample stage. The grounded Au counter probe attached to the micropositioner is penetrated through the SAM to contact and ground the metal film. Using the micromanipulator, the EGaIn tip is brought into contact with the SAM by using the CCD camera to monitor the proximity of the tip with its reflection on the metal film. Contact is indicated when the tip and its reflection converge.

To perform an $I(V)$ scan, the Keithley applies a bias, $V$, across the junction, with a positive value of $V$ corresponding to EGaIn being biased positively with respect to the metal film. ${ }^{[2}$ ${ }^{29]}$ In order to be able to conclude anything of significance, statistically relevant numbers of data must be collected for the molecular tunneling junctions. Therefore for each molecular junction current density $(J)$ is measured as a function of voltage $(V)$ across typically 20 individual EGaIn junctions, created over a minimum of 3 individual substrates. The maximum number of junctions measured on a single substrate, depends on the metal used and time that each measurement takes, i.e. Ag oxidizes in ambient conditions much faster than $\mathrm{Au}$. For each EGaIn junction 20 scans are measured \pm the desired $V$ range, for example $\pm 2.0 \mathrm{~V}$ with one scan consisting of a voltage sweep, $0.0 \mathrm{~V} \rightarrow+2.0 \mathrm{~V} \rightarrow 0.0 \mathrm{~V} \rightarrow-2.0 \mathrm{~V} \rightarrow$ $0.0 \mathrm{~V}$. For ease of data analysis, $I$ must be measured across consistent values of $V$ for each molecular tunneling junction, i.e. using a consistent voltage step size. In a majority of the data presented in this thesis, $I$ is measured every $0.1 \mathrm{~V}$ across a bias range of $\pm 2.0 \mathrm{~V}$, giving a minimum of 82 measurement points. 


\subsubsection{Statistical Data Analysis}

From the obtained data, average $|J|$ vs. $V$ scans are calculated by plotting the $|J|$ measured at each voltage value (in the example below the voltage step size is $0.1 \mathrm{~V}$, in both sweep directions) into histograms, giving in total 82 individual histograms corresponding to the number of times a particular value of $|J|$ was measured at that particular voltage (Figure 3.15). As the experimental values of $J$ are not normally distributed but log-normally distributed, the most relevant statistic for describing the distribution of $J$ is not the mean (arithmetic mean, with $N$ the number of values of $J$ [Eq. (3.1)]) which is biased toward high values of $J$, but the $\log$ mean (also known as the geometric mean [Eq. (3.2)]). ${ }^{[2-3,23-24,30]}$ Therefore, all the histograms were plotted on a log scale, giving a normal distribution, allowing the data to be fit with Gaussian curves (Figure 3.15b-e). This gave the average $|J|$ (the log mean) and the error (one-log standard deviation, 68\% of the data is within one $\log$ standard deviation of the log-mean) for each voltage value, allowing the construction of the average $|J|$ vs. $V$ scan (Figure 3.15a), with one single data point on the graph representing one Gaussian fit histogram.

$$
\begin{gathered}
\langle J\rangle=\frac{1}{N} \sum_{i=1}^{N} J i \\
\langle J\rangle \log =10^{z} \text { with } z=\frac{1}{N} \sum_{i=1}^{N} \log 10|J i|
\end{gathered}
$$


a) $10^{-2}$
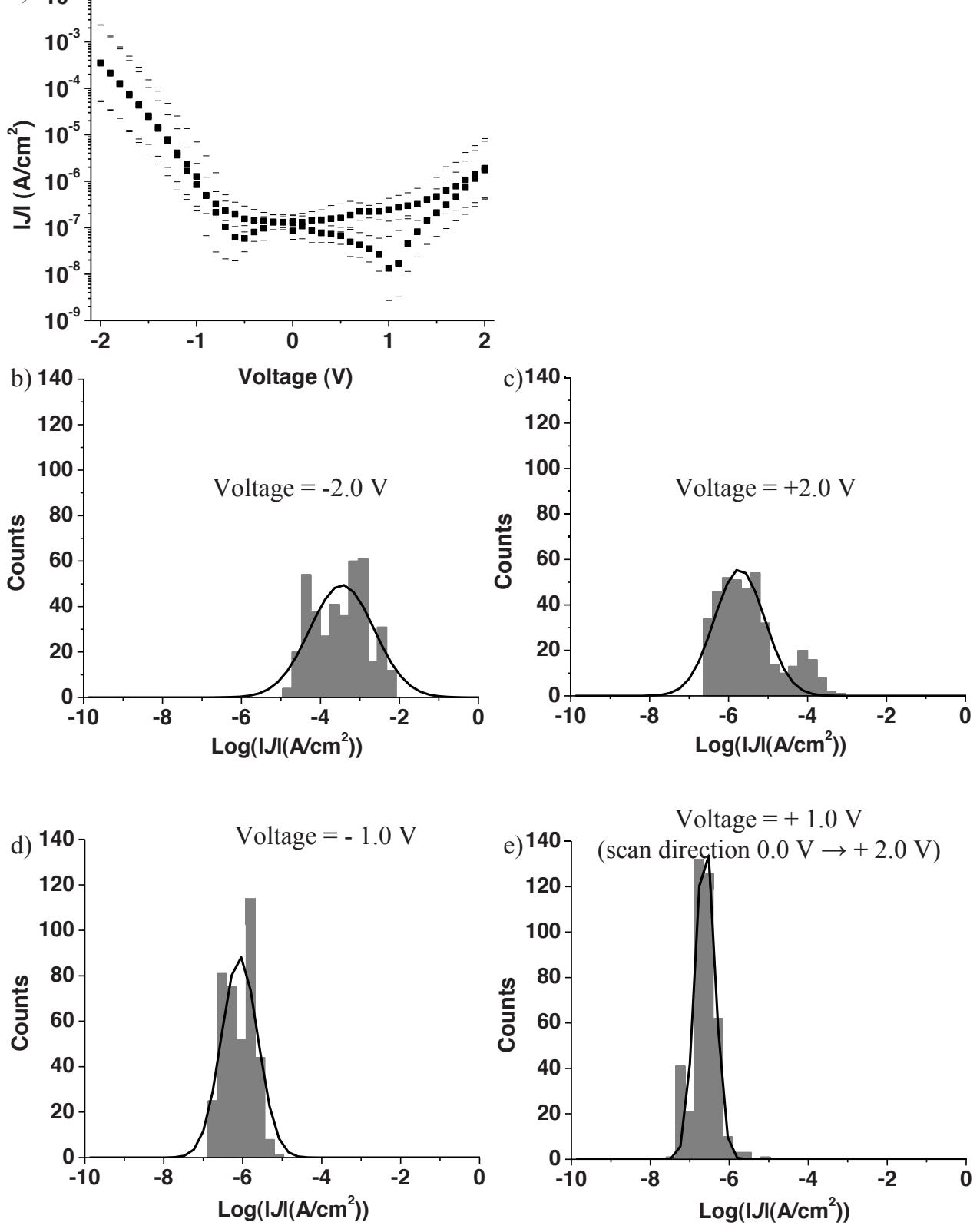

Figure 3.15: A semi-log plot of the averaged $|J|$ vs. $V$ for a EGaIn supramolecular tunneling junction (a), the statistical analysis for determining the averaged data point (log mean) at $-2.0 \mathrm{~V}$ (b), $+2.0 \mathrm{~V}$ (c), $-1.0 \mathrm{~V}(\mathrm{~d}),+1.0$ $\mathrm{V}(\mathrm{e})$. Shorts cannot be seen on this scale as they have current densities of approximately $10^{\wedge}$ (Figure reprinted with permission from. ${ }^{[29]}$ Copyright (C) 2010, John Wiley and Sons). 
The explanation for the log normal distribution of $J$, stems from the fact that tunneling current depends exponentially on the distance between the two electrodes. ${ }^{[31]}$ This is clearly shown in a simplified version of the Simmons equation [Eq. (3.3) $]^{[31 \mathrm{a}]}$ where $J\left(\mathrm{~A} / \mathrm{cm}^{2}\right)$ is current density, $d(\AA)$ is the distance between the two electrodes, $J_{0}$ is the current density flowing through the electrode-SAM interfaces in the hypothetical case of $d=0 \AA$ and $\beta\left(\AA^{-1}\right)$ is the tunneling decay constant). However, in an ideal case the value of $d$ is only determined by the thickness of the SAM, which in real junctions has a variety of defects present. ${ }^{[24,30]}$ As these defects create thin (where $d$ becomes smaller) and thick (where $d$ becomes larger) areas, they lead to a normal distribution of the value of $d$. Therefore as $J$ depends exponentially on a normally distributed variable, it is itself log normally distributed.

$$
J=J_{\mathrm{o}} \mathrm{e}^{-\beta \mathrm{d}}
$$

\subsubsection{Importance of Statistically Relevant Numbers of Data}

The importance of collecting statistically large amounts of data, stems from the fact that every molecular junction formed is different. This is mainly due to the presence of defects in the SAM formed on the metal film (Figure 3.16). ${ }^{[32]}$ These defects are caused by impurities in the metal film, and the SAM adsorbate solution and finally the structural defects present in the metal film itself, which will always be present to some extent no matter how much care is taken to make the substrates as flat as possible. In areas where defects are present, the packing and orientation of the SAM will change, and hence, also will the distance between the electrodes. ${ }^{[24,30]}$ Some defects will decrease the distance between the electrodes $(d)$ (thin area defects), and others will increase $d$, (thick area defects). As shown by the simplified version of the Simmons [Eq. (3.3) $]^{[29,31]} J$ depends exponentially on $d$. Therefore, as different types and different amounts of defects are present in each junction, it is essential to obtain statistically relevant numbers of data to average out the influence that the defects have on the obtained $J$ values. ${ }^{[2-3,10 b]}$ Additionally, because $J$ decays exponentially with increasing distance between the electrodes, the thin area defects can dominate the charge transport characteristics, leading to disproportionally high values of $J$ relative to the junction area, whereas thick area defects can only decrease the values of $J$ obtained proportionally to the junction area. However, by using the geometric mean [Eq. (3.2)] rather than the arithmetic mean [Eq. (3.1)] to average the accumulative $J$ values, the influence of the thin area defects can be somewhat minimized. ${ }^{[30]}$ 


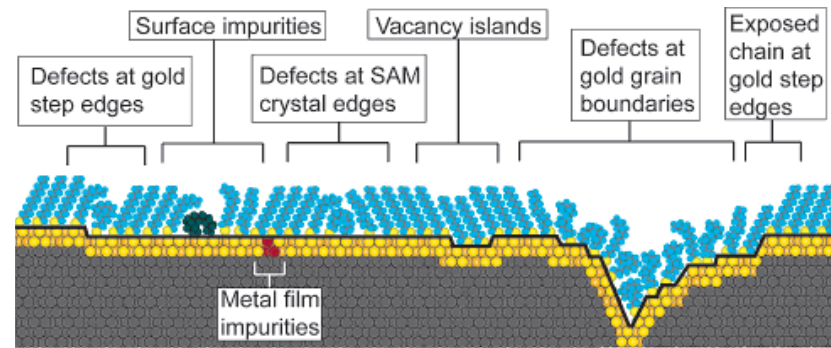

Figure 3.16: Defects in a SAM and on a metal substrate (Figure reprinted with permission from. ${ }^{[32]}$ Copyright (C) 2005, American Chemical Society).

\subsubsection{Minimizing Defects in Supramolecular Tunneling Junctions}

To minimize the defects present in the supramolecular tunneling junctions investigated in this thesis, the SAMs were formed on template-stripped $\mathrm{Au}$ surfaces $\left(\mathrm{Au}^{\mathrm{TS}}\right)$. The procedure to fabricate a TS surface is explained in Chapter 2 in section 2.2.1. In Chapter 4, the $\mathrm{Au}^{\mathrm{TS}}$ surfaces were fabricated using an UV-curable optical adhesive, as published by Whitesides and co-workers and were stated to produce $\mathrm{Au}^{\mathrm{TS}}$ with a root mean squared (RMS) roughness of $0.6 \mathrm{~nm}$ over an area of $25 \mathrm{\mu m}^{2}{ }^{[33]}$ When re-creating these $\mathrm{Au}^{\mathrm{TS}}$ at the University of Twente, these surfaces were found to visibly bubble upon immersion in $60^{\circ} \mathrm{C}$ ethanol. As heating the solution was an essential step to obtain high quality monolayers, $\mathrm{Au}^{\mathrm{TS}}$ were alternatively fabricated using a heat-curable optical adhesive, as published by Wrochem and co-workers. ${ }^{[34]}$ Wrochem and co-workers claimed that heating the adhesive at $150^{\circ} \mathrm{C}$ for 1 hour produced $\mathrm{Au}^{\mathrm{TS}}$ with a RMS roughness of $0.3 \mathrm{~nm},{ }^{[34]}$ however, they did not state over which area this value was obtained. This claim was tested by creating $\mathrm{Au}^{\mathrm{TS}}$ by heating the adhesive for $80^{\circ} \mathrm{C}$ for $\sim 20$ hours, $100^{\circ} \mathrm{C}$ for $\sim 5$ hours and $150^{\circ} \mathrm{C}$ for 1 hour. Once removed from the silicon wafer, these surfaces were immersed in $60^{\circ} \mathrm{C}$ ethanol overnight, to simulate the monolayer formation procedure. After rinsing with ethanol, and drying under a constant stream of $\mathrm{N}_{2}$, the roughness of these surfaces were investigated with AFM. Across an area of $25 \mu^{2}$, the RMS roughness of the $\mathrm{Au}^{\mathrm{TS}}$ created by heating the adhesive for $80^{\circ} \mathrm{C}$ for $\sim 20$ hours, $100^{\circ} \mathrm{C}$ for $\sim 5$ hours and $150^{\circ} \mathrm{C}$ for 1 hour were $0.3 \mathrm{~nm}, 0.3 \mathrm{~nm}$ and $0.9 \mathrm{~nm}$, respectively (Figure 3.17). Therefore, the temperature used by Wrochem and co-workers to cure the heat-curable adhesive was too high, and thus, the $\mathrm{Au}^{\mathrm{TS}}$ used in Chapters 5 and 6 were fabricated by curing the adhesive at $80^{\circ} \mathrm{C}$ for $\sim 20$ hours. 
a)
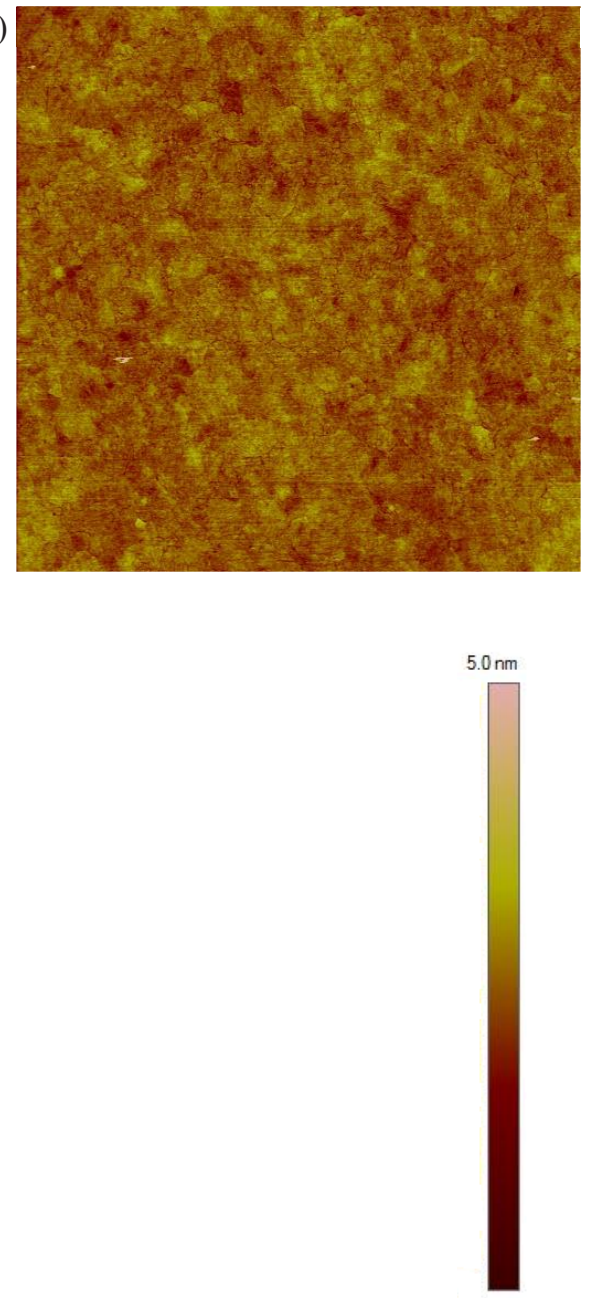

b)

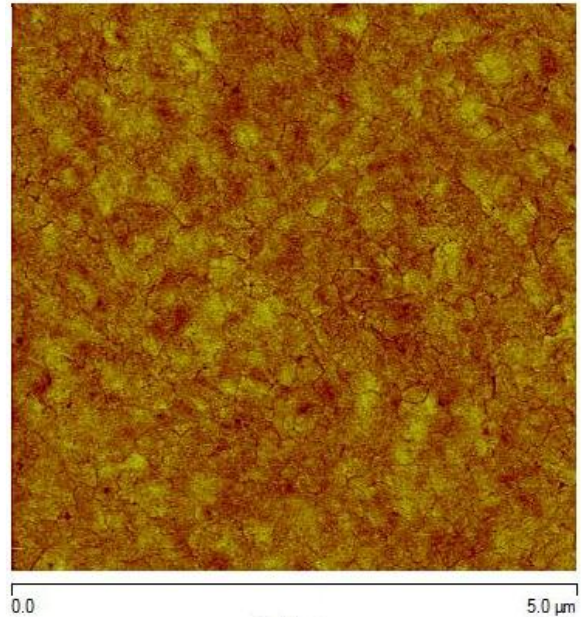

c)

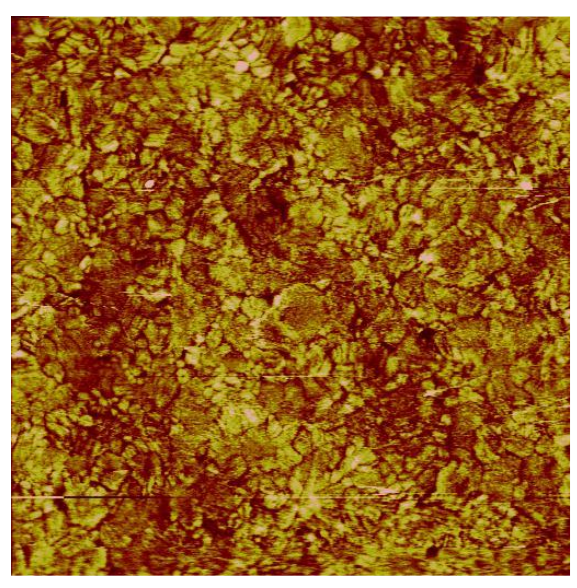

Figure 3.17: AFM images $5.0 \mu \mathrm{m}$ by $5.0 \mu \mathrm{m}$ of $\mathrm{Au}^{\mathrm{TS}}$ that were fabricated using the heat-curable optical adhesive, and subsequently after removal from the $\mathrm{SiO}_{2}$ wafer, were immersed in $60^{\circ} \mathrm{C}$ ethanol overnight. The $\mathrm{Au}^{\mathrm{TS}}$ were fabricated by heating the adhesive for $80^{\circ} \mathrm{C}$ for $\sim 20$ hours (a), $100^{\circ} \mathrm{C}$ for $\sim 5$ hours (b) and $150^{\circ} \mathrm{C}$ for 1 hour (c). Height scale for all AFM images is $5 \mathrm{~nm}$. 


\subsubsection{The Use of Rectification to Investigate $J(V)$ Characteristics}

Using rectification which is defined as $R=|J(-\mathrm{X} \mathrm{V})| /|J(+\mathrm{X} \mathrm{V})|$, (in this thesis $\mathrm{X}=2.0 \mathrm{~V}$ ) to investigate charge transport, has the advantage of using, within the same tunneling junction, the current measured at the forward bias as the reference for the current measured at the reverse bias. $^{[3,29]}$ This minimizes many of the uncertainties and complexities, such as contact resistances and contact areas, which are associated with comparing values of $J$ obtained from different tunneling junctions. Even the most carefully prepared SAM-based tunneling junctions can exhibit larger variations in $J$. Throughout this thesis $R$ is used to compare different supramolecular junctions, for the very reasons mentioned above. $R$ is analyzed in the same fashion as the $J$ measurements for each molecular junction and as previously published. ${ }^{[3]}$ The $R$ for each individual scan is calculated at $\pm 2.0 \mathrm{~V}$ for each supramolecular junction structure and plotted into histograms. As in the same case of $J, R$ is also log normally distributed, due to it being the ratio of $|J|$ at two opposing biases. Therefore, plotting the histograms on a log scale allowed them to be fit with single Gaussian functions that gave the log-mean value (average) $R$ and the log standard deviation (with all errors stated representing one log-standard deviation, $68 \%$ of the distribution of the data is within one log-standard deviation of the log-mean (Figure 3.18).

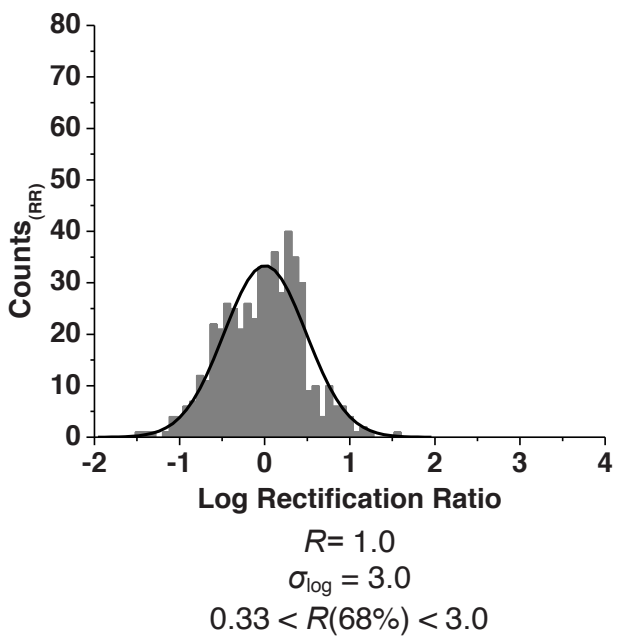

Figure 3.18: Example of a histogram of $\log$ Rectification ratio $(R)$ calculated $\pm 2.0 \mathrm{~V}$ for a supramolecular tunneling junction (Figure adapted with permission from. ${ }^{[29]}$ Copyright $(C) 2010$, John Wiley and Sons). 


\subsubsection{Reproducibility of Data.}

To determine if the data obtained for molecular tunneling junctions is reproducible on different EGaIn setups, three supramolecular tunneling junctions that were investigated using the EGaIn setup in the laboratory of Whitesides and co-workers (presented in Chapter 4 of this thesis) were also investigated in the EGaIn setup built at the University of Twente. Upon comparison, the $J(V)$ data and the values of $R$ determined were reproducible from setup to setup within experimental error (Figure 3.19) (For comparisons please refer to $J(V)$ data in Figure 4.3, and values of $R$ determined in Figure 4.4, in Chapter 4). 

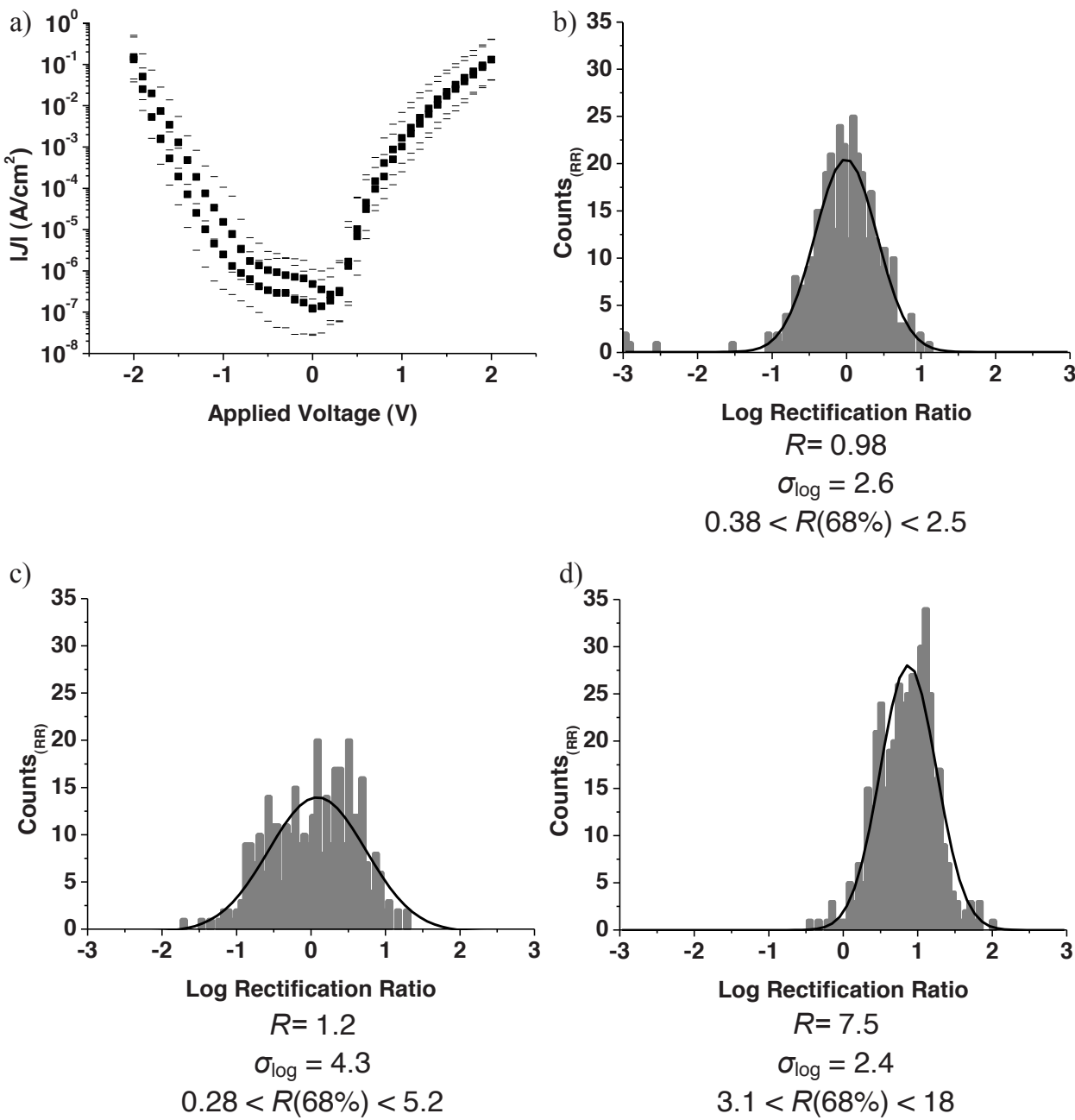

Figure 3.19: Examples of data obtained from $J(V)$ measurements performed on three different supramolecular tunneling junctions, in the EGaIn setup rebuilt at the University of Twente. The data is the same (within experimental error) of that obtained for the same supramolecular tunneling junctions in the EGaIn setup in the Laboratory of Whitesides and co-workers. (a) Semi $\log J(V)$ plot which should be compared to junction 4.4 in Chapter 4, histograms of $\log$ Rectification ratio $(R)$ calculated $\pm 2.0 \mathrm{~V}$, with (b) to be compared with junction $4.4\left(R=1.0, \sigma_{\log }=3.0\right)$, (c) to be compared with junction $4.3\left(R=0.7, \sigma_{\log }=2.5\right)$, (d) to be compared with junction $4.1\left(R=7.7, \sigma_{\log }=3.1\right)$ in Chapter 4 . 


\subsection{Conclusion}

In this Chapter, the advantages and disadvantages of the most common techniques used to create large area tunneling junctions were critically discussed. Subsequently, the successful construction of the EGaIn technique at the University of Twente was described, including all materials required, calibration measurements performed, complications and possible improvements. The system was found to be very sensitive, with a current offset of only $\sim 5$ pA, and very stable, allowing for $J(V)$ measurements to be regularly performed for a period of $\sim 2$ hours. The challenges faced with constructing and performing $J(V)$ measurements using this EGaIn set-up are discussed in order to optimize 'future' setups and to help understand small anomalies that may occur in the $J(V)$ data obtained. Subsequently, a thorough example of how to create an EGaIn junction and how to collect and analyze statistically relevant numbers of data was given, along with a discussion of why statistical relevant numbers of data are necessary. Also, $I(V)$ or $J(V)$ measurements performed on supramolecular tunneling junctions using the EGaIn setup in the laboratory of Whitesides and co-workers were found to be reproducible in the EGaIn setup at the University of Twente. Therefore, by referring to this Chapter a prospective user can re-construct this setup and use the EGaIn technique to investigate charge transport across molecular assemblies. Finally, as the EGaIn technique is the basis technique in this thesis, this chapter will have allowed for a greater understanding of the experiments undertaken and data presented in the upcoming Chapters of 4, 5 and 6. 


\subsection{Experimental Details}

Table 3.1: Components needed to build the EGaIn technique.

\begin{tabular}{|c|c|c|}
\hline Amount & Apparatus + all components ordered & Company \\
\hline & Breadboard and general components & \\
\hline 1 & NT55-251: Metric composite breadboard 600X900 $\mathrm{mm}$ (optic table) & Edmund Optics \\
\hline 1 & NT54-262: 3/4" Post Breadboard Adapter & Edmund Optics \\
\hline 1 & NT59-763: Steel post. $152.4 \mathrm{~mm}$ m6stud & Edmund Optics \\
\hline 2 & NT56-923: Sorbothane Bumpers M6 (pkg of 4) & Edmund Optics \\
\hline 2 & NT55-382: 5" square metric adapter & Edmund Optics \\
\hline 6 & NT58-987: Thread adapter m6-m6 & Edmund Optics \\
\hline \multirow[t]{2}{*}{1} & NT55-193: M6x1.0 x25mm SCHS, 50 pack & Edmund Optics \\
\hline & CCD Camera & \\
\hline 1 & NT55-834: VZM ${ }^{\mathrm{TM}} 1000 \mathrm{i}$ and Complete VZM ${ }^{\mathrm{TM}} 1000 \mathrm{i}$ Color System & Edmund Optics \\
\hline 1 & NT-57-291: Hitachi KP-D20B Color CCD Camera & Edmund Optics \\
\hline 1 & NT54-644: Cable Y-C(S-VIDEO) 12' Long Type Y-C (S-Video), Length 12' RoHS compliant & Edmund Optics \\
\hline \multirow[t]{2}{*}{1} & NT39-930: X-Y Positioning stage & Edmund Optics \\
\hline & Illuminating Light & \\
\hline 1 & NT59-236: 220V, MI-150 Fiber opticilluminator & Edmund Optics \\
\hline 1 & NT54-210: Single Branch Semi-rigid Light Guide & Edmund Optics \\
\hline \multirow[t]{2}{*}{1} & NT38-946: 0.565" ID fiber optic light guide adapter & Edmund Optics \\
\hline & Micropositioner & \\
\hline 1 & S-725PLM: Micropositioner left handed & Sel-Tek \\
\hline \multirow[t]{2}{*}{1} & U-P: Universal probeholder & Sel-Tek \\
\hline & Au coated probes & American Probes and Tech. \\
\hline \multirow[t]{3}{*}{1} & Metal disc platform & Internal \\
\hline & & \\
\hline & Micromanipulator & \\
\hline 1 & YOU-1: XYZ-manipulator & Narishige International \\
\hline \multirow[t]{3}{*}{1} & IP: Iron plate for magnetic stand & Narishige International \\
\hline & Syringes & Hamiltion \\
\hline & Sample Stage & \\
\hline 1 & MBT616/M: MicroblockXYZ & Thorlabs \\
\hline \multirow[t]{2}{*}{1} & RB13P1/M: Breadboard Adapter Plate for MicroBlock Stages & Thorlabs \\
\hline & Current-Voltage source and measurement & \\
\hline \multirow[t]{2}{*}{1} & Model 6430 Sub-femtoamp Remote Source Meter & Keithley Instruments \\
\hline & Specific Connectors & \\
\hline 1 & NI GPIB-USB-HS (GPIB Controller for Hi Speed USB) & National Instruments \\
\hline \multirow[t]{5}{*}{1} & 000-000015: External video G x rabber + usb cable & Opto Sonderbedarf \\
\hline & & GMBH \\
\hline & & \\
\hline & Labview & Internal \\
\hline & VR Magic Camlab & Edmund Optics \\
\hline
\end{tabular}




\subsection{References}

[1] a)H. Song, M. A. Reed, T. Lee, Adv. Mater. 2011, 23, 1583-1608; b)R. L. McCreery, A. J. Bergren, Adv. Mater. 2009, 21, 4303-4322; c)H. B. Akkerman, B. de Boer, J. Phys.: Condens. Matter 2008, 20, 013001.

[2] R. C. Chiechi, E. A. Weiss, M. D. Dickey, G. M. Whitesides, Angew. Chem. Int. Ed. 2008, 47, 142-144.

[3] C. A. Nijhuis, W. F. Reus, G. M. Whitesides, J. Am. Chem. Soc. 2009, 131, 17814-17827.

[4] F. Chen, J. Hihath, Z. F. Huang, X. L. Li, N. J. Tao, in Annu. Rev. Phys. Chem., Vol. 58, Annual Reviews, Palo Alto, 2007, pp. 535-564.

[5] a)L. A. Bumm, J. J. Arnold, T. D. Dunbar, D. L. Allara, P. S. Weiss, J. Phys. Chem. B 1999, 103, 8122-8127; b)X. D. Cui, A. Primak, X. Zarate, J. Tomfohr, O. F. Sankey, A. L. Moore, T. A. Moore, D. Gust, G. Harris, S. M. Lindsay, Science 2001, 294, 571-574; c)A.-S. Hallbäck, B. Poelsema, H. J. W. Zandvliet, Solid State Commun. 2007, 141, 645-648; d)B. Venkataraman, J. J. Breen, G. W. Flynn, Scanning Tunneling Microscopy Studies of Alcohol Alkane Mixtures Adsorbed on Graphite Surfaces, Plenum Press Div Plenum Publishing Corp, New York, 1994.

[6] a)D. J. Wold, C. D. Frisbie, J. Am. Chem. Soc. 2001, 123, 5549-5556; b)V. B. Engelkes, J. M. Beebe, C. D. Frisbie, J. Am. Chem. Soc. 2004, 126, 14287-14296; c)G. Wang, T. W. Kim, G. Jo, T. Lee, J. Am. Chem. Soc. 2009, 131, 5980-5985.

[7] a)T. Lee, W. Y. Wang, J. F. Klemic, J. J. Zhang, J. Su, M. A. Reed, J. Phys. Chem. B 2004, 108, 8742-8750; b)M. A. Reed, C. Zhou, C. J. Muller, T. P. Burgin, J. M. Tour, Science 1997, 278, 252-254; c)W. Y. Wang, T. Lee, M. A. Reed, J. Phys. Chem. B 2004, 108, 18398-18407.

[8] a)J. G. Kushmerick, D. B. Holt, J. C. Yang, J. Naciri, M. H. Moore, R. Shashidhar, Phys. Rev. Lett. 2002, 89, 086802; b)J. G. Kushmerick, D. B. Holt, S. K. Pollack, M. A. Ratner, J. C. Yang, T. L. Schull, J. Naciri, M. H. Moore, R. Shashidhar, J. Am. Chem. Soc. 2002, 124, 1065410655.

[9] a)C. Zhou, M. R. Deshpande, M. A. Reed, L. Jones li, J. M. Tour, Appl. Phys. Lett. 1997, 71, 611-613; b)J. Chen, M. A. Reed, A. M. Rawlett, J. M. Tour, Science 1999, 286, 1550-1552.

[10] a)R. L. McCreery, J. Wu, R. P. Kalakodimi, Phys. Chem. Chem. Phys. 2006, 8, 2572-2590; b)T.W. Kim, G. Wang, H. Lee, T. Lee, Nanotechnology 2007, 18, 315204; c)A. Vilan, D. Cahen, Adv. Funct. Mater. 2002, 12, 795-807; d)K. T. Shimizu, J. D. Fabbri, J. J. Jelincic, N. A. Melosh, Adv. Mater. 2006, 18, 1499-1504; e)H. B. Akkerman, P. W. M. Blom, D. M. de Leeuw, B. de Boer, Nature 2006, 441, 69-72; f)K. Slowinski, H. K. Y. Fong, M. Majda, J. Am. Chem. Soc. 1999, 121, 7257-7261; g)R. Haag, M. A. Rampi, R. E. Holmlin, G. M. Whitesides, J. Am. Chem. Soc. 1999, 121, 7895-7906; h)Y. L. Loo, D. V. Lang, J. A. Rogers, J. W. P. Hsu, 
Nano Lett. 2003, 3, 913-917; i)G. Wang, Y. Kim, M. Choe, T. W. Kim, T. Lee, Adv. Mater. 2011, 23, 755-760; j)S. Seo, M. Min, J. Lee, T. Lee, S.-Y. Choi, H. Lee, Angew. Chem. Int. Ed. 2012, 51, 108-112.

[11] a)R. E. Holmlin, R. Haag, M. L. Chabinyc, R. F. Ismagilov, A. E. Cohen, A. Terfort, M. A. Rampi, G. M. Whitesides, J. Am. Chem. Soc. 2001, 123, 5075-5085; b)R. E. Holmlin, F. I. Rustem, H. Rainer, M. Vladimiro, A. R. Mark, R. Maria Anita, M. W. George, Angew. Chem. Int. Ed. 2001, 40, 2316-2320.

[12] E. A. Speets, B. J. Ravoo, F. J. G. Roesthuis, F. Vroegindeweij, D. H. A. Blank, D. N. Reinhoudt, Nano Lett. 2004, 4, 841-844.

[13] V. V. Zhirnov, R. K. Cavin, Nature Mater. 2006, 5, 11-12.

[14] Y. L. Loo, R. L. Willett, K. W. Baldwin, J. A. Rogers, J. Am. Chem. Soc. 2002, 124, 7654-7655.

[15] H. Haick, D. Cahen, Acc. Chem. Res. 2008, 41, 359-366.

[16] A. J. Kronemeijer, H. B. Akkerman, T. Kudernac, B. J. van Wees, B. L. Feringa, P. W. M. Blom, B. de Boer, Adv. Mater. 2008, 20, 1467-1473.

[17] P. A. Van Hal, E. C. P. Smits, T. C. T. Geuns, H. B. Akkerman, B. C. De Brito, S. Perissinotto, G. Lanzani, A. J. Kronemeijer, V. Geskin, J. Cornil, P. W. M. Blom, B. De Boer, D. M. De Leeuw, Nature Nanotech. 2008, 3, 749-754.

[18] a)G. Wang, H. Yoo, S. I. Na, T. W. Kim, B. Cho, D. Y. Kim, T. Lee, Thin Solid Films 2009, 518, 824-828; b)A. J. Kronemeijer, I. Katsouras, E. H. Huisman, P. A. van Hal, T. C. T. Geuns, P. W. M. Blom, D. M. de Leeuw, Small 2011, 7, 1593-1598.

[19] B. C. de Brito, E. C. P. Smits, P. A. van Hal, T. C. T. Geuns, B. de Boer, C. J. M. Lasance, H. L. Gomes, D. M. de Leeuw, Adv. Mater. 2008, 20, 3750-3753.

[20] A. J. Kronemeijer, E. H. Huisman, I. Katsouras, P. A. van Hal, T. C. T. Geuns, P. W. M. Blom, S. J. van der Molen, D. M. de Leeuw, Phys. Rev. Lett. 2010, 105, 156604.

[21] a)M. A. Rampi, G. M. Whitesides, Chem. Phys. 2002, 281, 373-391; b)K. Slowinski, M. Majda, J. Electroanal. Chem. 2000, 491, 139-147.

[22] a)I. Levine, S. M. Weber, Y. Feldman, T. Bendikov, H. Cohen, D. Cahen, A. Vilan, Langmuir 2012, 28, 404-415; b)F. Thieblemont, O. Seitz, A. Vilan, H. Cohen, E. Salomon, A. Kahn, D. Cahen, Adv. Mater. 2008, 20, 3931-3936.

[23] C. A. Nijhuis, W. F. Reus, J. R. Barber, M. D. Dickey, G. M. Whitesides, Nano Lett. 2010, 10, 3611-3619.

[24] C. A. Nijhuis, W. F. Reus, G. M. Whitesides, J. Am. Chem. Soc. 2010, 132, 18386-18401.

[25] M. M. Thuo, W. F. Reus, C. A. Nijhuis, J. R. Barber, C. Kim, M. D. Schulz, G. M. Whitesides, J. Am. Chem. Soc. 2011, 133, 2962-2975. 
[26] A. Salomon, T. Boecking, O. Seitz, T. Markus, F. Amy, C. Chan, W. Zhao, D. Cahen, A. Kahn, Adv. Mater. 2007, 19, 445-450.

[27] In the laboratory of Whitesides and co-workers, the EGaln technique is suspended from the roof.

[28] Model 6430 Sub-Femtoamp Remote SourceMeter Instruction Manual, Vol. 4, Keithley Instruments, Inc., Cleveland, 2001.

[29] K. S. Wimbush, W. F. Reus, W. G. van der Wiel, D. N. Reinhoudt, G. M. Whitesides, C. A. Nijhuis, A. H. Velders, Angew. Chem. Int. Ed. 2010, 49, 10176-10180.

[30] E. A. Weiss, R. C. Chiechi, G. K. Kaufman, J. K. Kriebel, Z. Li, M. Duati, M. A. Rampi, G. M. Whitesides, J. Am. Chem. Soc. 2007, 129, 4336-4349.

[31] a)J. G. Simmons, J. Appl. Phys. 1963, 34, 1793-1803; b)C. Joachim, M. A. Ratner, Proc. Natl. Acad. Sci. U. S. A. 2005, 102, 8801-8808.

[32] J. C. Love, L. A. Estroff, J. K. Kriebel, R. G. Nuzzo, G. M. Whitesides, Chem. Rev. 2005, 105, 1103-1169.

[33] E. A. Weiss, G. K. Kaufman, J. K. Kriebel, Z. Li, R. Schalek, G. M. Whitesides, Langmuir 2007, 23, 9686-9694.

[34] D. Q. Gao, F. Scholz, H. G. Nothofer, W. E. Ford, U. Scherf, J. M. Wessels, A. Yasuda, F. von Wrochem, J. Am. Chem. Soc. 2011, 133, 5921-5930. 


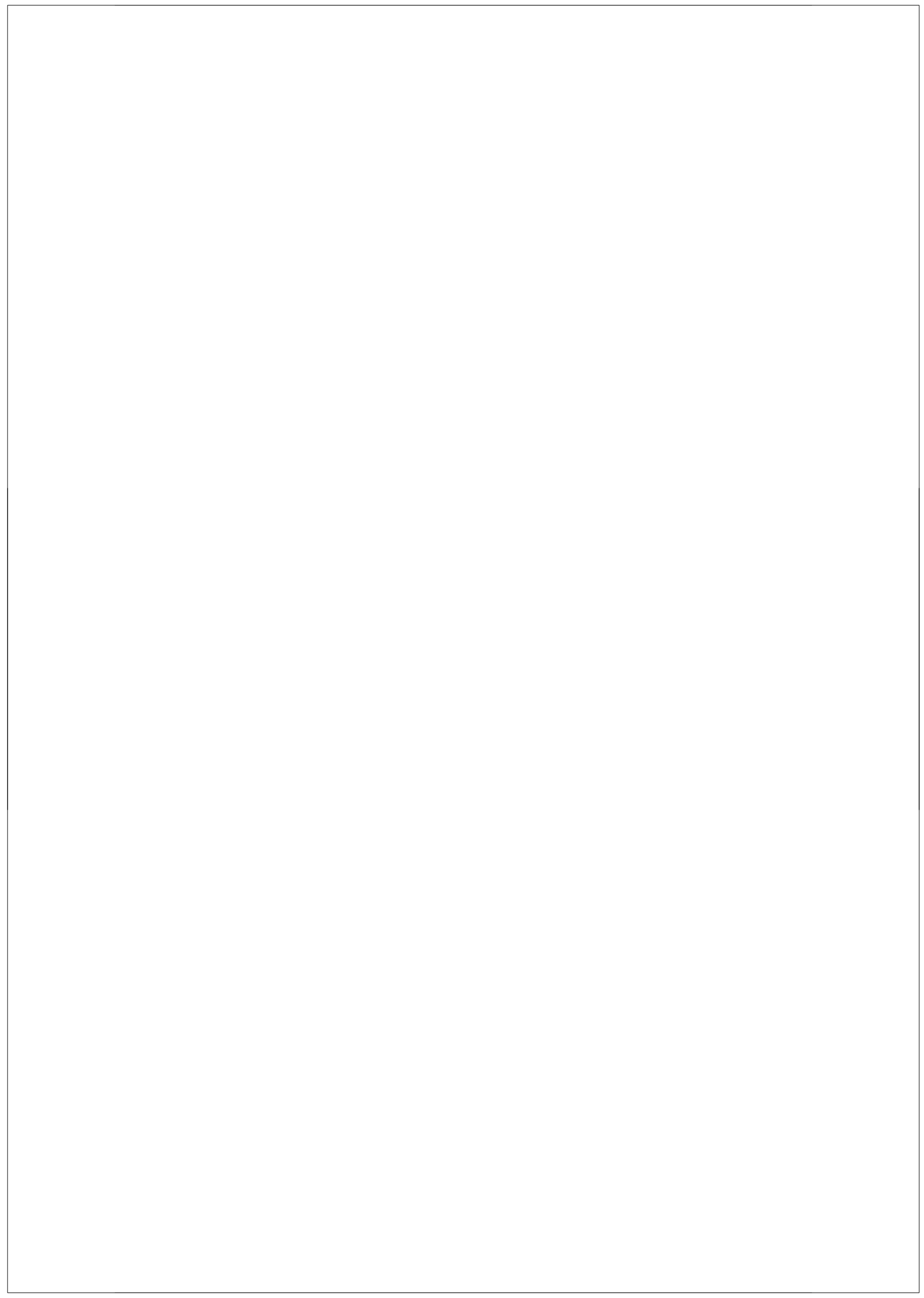




\section{Control Over Rectification in}

\section{Supramolecular Tunneling Junctions}

Supramolecular interactions on surfaces have an advantage over chemical (covalent) modification, as they allow self-correction, are specific and directional and are well defined, which implies that the binding properties can be manipulated with great precision. Here, well-defined supramolecular interactions, of a variety of generation-one guest dendrimers with template stripped $\mathrm{Au}$ immobilized $\beta$-cyclodextrin $(\beta \mathrm{CD})$ host molecules, have been exploited in Eutectic Gallium Indium (EGaIn) tunneling junctions. Control of the rectification in these junctions was achieved by varying the terminal functionality of poly(propylene) imine (PPI) dendrimers from adamantyl (Ad) to ferrocene $(\mathrm{Fc})$ and biferrocene $(\mathrm{BFc})$.

*This chapter was published as K. S. Wimbush, W. F. Reus, W. G. van der Wiel, D. N. Reinhoudt, G. M. Whitesides, C. A. Nijhuis, A. H. Velders, Angew. Chem. Int. Ed. 2010, 49, 10176-10180, and is adapted with permission. Copyright (C) 2010 John Wiley and Sons. 


\subsection{Introduction}

Reported herein is the concept of using a supramolecular platform on which poly(propylene) imine (PPI) dendrimers can be immobilized to result in tunneling junctions formed from assemblies with well-defined structures. In this way, the rectification can be controlled by changing only the chemical structure of the termini of the PPI dendrimers, while minimizing the changes of the whole supramolecular assemblies. This method makes it possible to perform physical-organic studies of charge transport across self-assembled monolayer (SAM)-based junctions. These junctions were fabricated using ultra-flat template-stripped $\mathrm{Au}$ bottom-electrodes $\left(\mathrm{Au}^{\mathrm{TS}}\right)$ and liquid metal top-electrodes of an eutectic alloy of $\mathrm{Ga}$ and In (EGaIn), with its superficial layer of $\mathrm{Ga}_{2} \mathrm{O}_{3}$. Junctions with monolayers of PPI dendrimers possessing terminal moieties with accessible highest occupied molecular orbital (HOMO) levels, viz. ferrocene $(\mathrm{Fc})$, immobilized on a supramolecular platform of a self-assembled monolayer (SAM) of $\beta$-cyclodextrin $(\beta C D)$, rectified currents with rectification ratios $(R$; at $\pm 2.0 \mathrm{~V})$ having a log-mean $\left(\mu_{\log }\right)$ of $\sim 1.7 \times 10^{2}$ and a log standard deviation ${ }^{[1]}\left(\sigma_{\log }\right)$ of $\sim 1.9$ [Eq. (4.1); $J=$ current density $\left(\mathrm{A} / \mathrm{cm}^{2}\right)$ and $V=$ voltage $\left.(\mathrm{V})\right]$. In contrast the junctions with monolayers of PPI dendrimers possessing terminal moieties without accessible HOMO levels, viz. adamantyl, did not rectify currents $\left(R=0.70 ; \sigma_{\log }=2.5\right)$, nor did the bare supramolecular platform, $\left(R=1.0 ; \sigma_{\log }=3.0\right)$. These experiments show that the rectification is dependent on the chemical structures of the molecules in these SAM-based junctions and that the rectification does not originate from any of the other asymmetries in the junctions or the $\mathrm{Ga}_{2} \mathrm{O}_{3}$ layer. ${ }^{[2]}$

$$
R=|J(-2.0 \mathrm{~V})| /|J(+2.0 \mathrm{~V})|
$$

A fundamental understanding of the mechanism of charge transport in tunneling junctions is important in molecular electronics, and more broadly, in understanding charge transport through organic matter ${ }^{[3]}$ in biochemistry, ${ }^{[3 \mathrm{a}]}$ energy harvesting, ${ }^{[3 \mathrm{~b}]}$ information storage, ${ }^{[3 \mathrm{c}, 3 \mathrm{~d}]}$ and sensing ${ }^{[3 e]}$. Studies of charge transport across SAM-based junctions, however, have been troublesome because an "ideal" technique to contact SAMs without forming shorts, ${ }^{[4]}$ filaments of metal, ${ }^{[5]}$ or altering the molecular and supramolecular structure of the SAMs, ${ }^{[5 a}$ ${ }^{5 b]}$ with a high yield of working devices, without defects caused by surface roughness of the electrodes, impurities, pin holes, step edges, or grains, ${ }^{[6]}$ is not available. Indeed, often "working" junctions or devices are ill-defined terms. Lee et $a 1,{ }^{[4 a, 4 c]}$ recognized these 
problems and analyzed large numbers of data statistically to determine yields in working devices, reproducibility, and the mechanism of charge transport in their devices. Thus it is postulated that physical-organic studies with statistically large numbers of data without "selecting" data, are required to account for defects in the junctions, discriminate artifactual data from real data, ${ }^{[7]}$ and to determine that the mechanism of charge transport across these junctions, and the electrical characteristics, such as tunneling, switching or rectification, of the junctions, are dominated by the molecules inside the junctions. ${ }^{[2,8]}$

Molecular rectification ${ }^{[9]}$ has been reported for molecules in a variety of molecular tunneling junctions. ${ }^{[10]}$ These studies as a group could not unambiguously determine the mechanism of charge transport, or that the rectification was caused by the SAMs inside the junctions, for four main reasons. First, poorly defined structures of the SAMs make it difficult to study the mechanism of charge transport as a function of molecular structure. ${ }^{[10 \mathrm{~b}, 11]}$ Second, physicalorganic studies with statistically large numbers of data have not been performed. Third, to acquire large numbers of data has been difficult due to the instability, low yield and low reproducibility of the molecular junctions. ${ }^{[4 \mathrm{a}]}$ Finally, some experimental data can be misleading, as molecular junctions that have their top- and bottom-electrodes fabricated from different materials, or have other asymmetries that are not related to the chemical structure of the SAM, have the possibility of rectifying in the absence of any structural or asymmetric organic components. ${ }^{[12]}$

Through the recent development of the $\left(\mathrm{Ga}_{2} \mathrm{O}_{3}\right)$ EGaIn technique, ${ }^{[13]}$ the difficulty in forming stable, reproducible, 'high' yielding molecular tunneling junctions that allow for the accumulation of statistically large numbers of data, has been addressed. This technique allowed for a charge transport investigation on SAMs of ferrocene functionalized alkanethiolates, on template stripped silver $\left(\mathrm{Ag}^{\mathrm{TS}}\right)$, with a $\left(\mathrm{Ga}_{2} \mathrm{O}_{3}\right)$ EGaIn top electrode. The Fc functionalized alkanethiolates were found to rectify currents with $R=1.0 \times 10^{2}$ $\left(\sigma_{\log }=3.0\right)$ at $\pm 1.0 \mathrm{~V} .^{[2]}$

Supramolecular tunneling junctions were fabricated consisting of SAMs of PPI dendrimers with different termini multivalently adsorbed on a supramolecular platform, i.e., the $\beta \mathrm{CD}$ SAM. These supramolecular systems were synthesized and characterized ${ }^{[14]}$ with atomic force microscopy (AFM), ${ }^{[14 \mathrm{~b},}{ }^{14 \mathrm{c},}{ }^{14 \mathrm{j}]}$ scanning tunneling microscopy (STM), ${ }^{[14 \mathrm{i}]}$ electrochemistry (cyclic voltammetry $(\mathrm{CV}),{ }^{[14 \mathrm{~b},}{ }^{14 \mathrm{e}-\mathrm{h}]}$ electrochemical impedance spectroscopy (EIS), ${ }^{[14 \mathrm{~b},}{ }^{14 \mathrm{~g}]}$ differential pulse voltammetry (DPV), ${ }^{[14 \mathrm{f}-\mathrm{h}]}$ scanning 
electrochemical microscopy $(\mathrm{SEM}),{ }^{[14 \mathrm{k}]}$ surface plasmon resonance $(\mathrm{SPR})^{[14 \mathrm{c}, 14 \mathrm{f}, 14 \mathrm{~h}]}$ and SPR and CV combined, ${ }^{[14 \mathrm{f}, 14 \mathrm{~h}]}$ to determine the thickness, molecular orientation and packing density of the supramolecular platform itself, and the coverage and conformation of the adsorbed PPI dendrimers (including the number of interactions of the dendrimer with the $\beta C D$ SAM; Table 1). In addition, Thompson ${ }^{[15]}$ modeled (with molecular dynamics) the adsorption of the Fc PPI dendrimers on the supramolecular platform and his findings support our conclusions. Thus, the supramolecular printboard provides optimal control over kinetics and thermodynamics resulting in well-defined supramolecular structures immobilized at the Au electrodes (Figure 4.1). As supramolecular interactions such as these ( $\beta C D+$ guest) have been reported to be stable in a dry state over prolonged periods (i.e., more than six months), ${ }^{[16]}$ we extend the use of the supramolecular platform to perform studies of charge transport across SAMs as a function of chemical composition of the SAM and to prove that the electrical characteristics are molecular in origin.

\subsection{Construction of the Supramolecular Tunneling Junctions: Generation One Poly(propylene) imine Dendrimers}

Figure 4.1 shows the construction of the supramolecular tunneling junctions. A welldefined, hexagonally packed SAM of heptathioether-functionalized $\beta$-cyclodextrin $(\beta C D)^{[14 a, 14 b]}$ is formed on $\mathrm{Au}^{\mathrm{TS}}$, thus creating the supramolecular platform. $\mathrm{Au}^{\mathrm{TS}}$ are used as the root mean square (RMS) roughness of these surfaces is 5 times less than that of evaporated surfaces, ${ }^{[17]}$ thus ensuring the optimization of working devices. The use of dendrimers as guest molecules, allows for multivalent host-guest interactions with the supramolecular platform to increase the stability of the supramolecular structures (single $\beta C D-F c$ interactions are too weak to obtain a fully covered $\beta C D$ SAM with native $F c$ ). Absorbed on this platform were PPI dendrimer guest molecules with three different terminal functionalities: 1) ferrocene (Fc), 2) bi-ferrocene (BFc), and 3) adamantyl (Ad). The Fc and $\mathrm{BFc}$ functionalized PPI dendrimers ${ }^{[18]}$ are important for providing molecular orbitals that are spatially asymmetrically located within the junction and energetically accessible (see below), and the Ad-functionalized dendrimers serve as a control. After dendrimer immobilization, or directly after the formation of the supramolecular platform (depending on the desired supramolecular junction) the top contact was applied using the $\left(\mathrm{Ga}_{2} \mathrm{O}_{3}\right)$ EGaIn technique. ${ }^{[13]}$ 


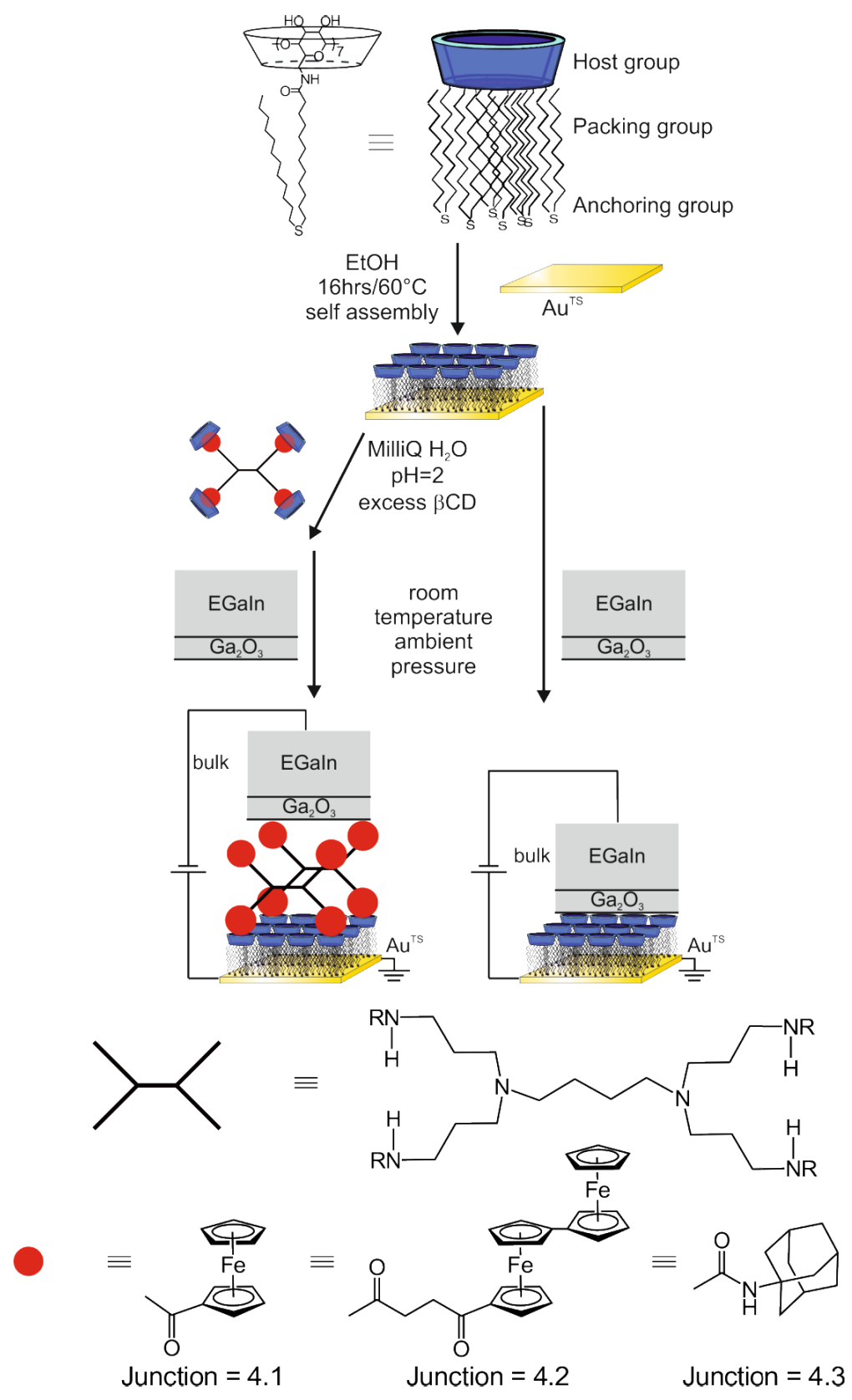

Figure 4.1: Schematic of the fabrication process of the molecular junctions. The eutectic gallium-indium (EGaIn) top contact is represented exhibiting its inherent gallium oxide layer. Molecular structure of the core of the poly(propylene) imine dendrimers, with the red sphere representing the functional groups at the periphery of the dendrimer, with the functionality of the dendrimer corresponding to junctions 4.1, 4.2 and 4.3, respectively. The $J(V)$ measurements were performed by biasing the $\left(\mathrm{Ga}_{2} \mathrm{O}_{3}\right) \mathrm{EGaIn}$ top-electrode and connecting the Au bottom-electrode to ground. 
All junctions are described using nomenclature, $\mathrm{Au}^{\mathrm{TS}}-\beta \mathrm{CDSAM} / \mathrm{X} /\left(\mathrm{Ga}_{2} \mathrm{O}_{3}\right) \mathrm{EGaIn}$ where "-" in $\mathrm{Au}^{\mathrm{TS}}-\beta \mathrm{CD}$ represents the non-covalent interface of the Au surface and the sulfur of the heptathioether functionalized $\beta C D$, “" represents the supramolecular host-guest interaction between the $\beta C D$ and the terminal functional group of the PPI dendrimer, " $\mathrm{X}$ " represents the PPI dendrimer, " $\left(\mathrm{Ga}_{2} \mathrm{O}_{3}\right)$ " represents the oxides of gallium present on the skin of the EGaIn and "///represents the Van der Waals interactions at the interface between the terminal group of the molecular structure and the $\mathrm{Ga}_{2} \mathrm{O}_{3}$ on the skin of the EGaIn.

Four different types of tunneling junctions were investigated. Two supramolecular junctions contained Fc moieties: one junction consisted of a generation one $\mathrm{Fc}$ functionalized poly(propylene) imine dendrimer $\left(\mathrm{G} 1-\mathrm{PPI}-(\mathrm{Fc})_{4}\right)$ immobilized on a $\beta \mathrm{CD}$ monolayer, $\mathrm{Au}^{\mathrm{TS}}$ $\beta C D S A M / G 1-P P I-(F c)_{4} / /\left(\mathrm{Ga}_{2} \mathrm{O}_{3}\right)$ EGaIn (4.1), and the other consisted of a G1-PPI-(BFc $)_{4}$ functionalized dendrimer immobilized on a $\beta C D$ monolayer, $\mathrm{Au}^{\mathrm{TS}}-\beta C D S A M / G 1-P P I-$ $(\mathrm{BFc})_{4} / /\left(\mathrm{Ga}_{2} \mathrm{O}_{3}\right) \mathrm{EGaIn}$ (4.2). Two other junctions did not contain ferrocene moieties: one junction consisted of a G1-PPI-(Ad) 4 functionalized dendrimer immobilized on a $\beta C D$ monolayer, $\mathrm{Au}^{\mathrm{TS}}-\beta C D S A M / G 1-P P I-(\mathrm{Ad})_{4} / /\left(\mathrm{Ga}_{2} \mathrm{O}_{3}\right)$ EGaIn (4.3) and the other was simply the bare $\beta C D$ monolayer, $\mathrm{Au}^{\mathrm{TS}}-\beta \mathrm{CDSAM} / /\left(\mathrm{Ga}_{2} \mathrm{O}_{3}\right) \mathrm{EGaIn}$ (4.4) (Figure 4.1). Junctions 4.3 and 4.4 served as "control" junctions to ensure that any possible characteristic trends seen in the $J(V)$ measurements of junctions 4.1 and 4.2 were attributed to the $\mathrm{Fc}$ or BFc moieties and did not arise from the supramolecular platform or the additional structural asymmetry created within the supramolecular tunneling junction by absorbing a dendrimer to the platform.

\subsection{Results and Discussion}

Presented and discussed throughout this section are the $J(V)$ data accumulation and statistical analysis, supramolecular rectification, mechanism of charge transport and finally the current density and hysteresis.

\subsection{1 $J(V)$ Data Accumulation and Statistical Analysis}

The $\left(\mathrm{Ga}_{2} \mathrm{O}_{3}\right)$ EGaIn electrodes are large on the molecular scale $\left(100\right.$ to $\left.300 \mu \mathrm{m}^{2}\right)$, ${ }^{[13]}$ therefore these so-called large area tunneling junctions contain in the order of $10^{6}-10^{7}$ molecules within the junction. The junctions in this study, as do all large area junctions, contain defects that arise, for example, from surface roughness of the electrodes (step edges, 
grains), defects in the SAMs, impurities. ${ }^{[6-7]}$ The amount and types of defects vary from junction to junction and substrate to substrate, so it is essential to collect a statistically large amount of data from different $\mathrm{Au}^{\mathrm{TS}}$ substrates in order to conclude anything of significance. ${ }^{[4 a]}$ Therefore, in this study large numbers of data were collected (Table 4.1) allowing for a statistical analysis similar to that reported by Whitesides and co-workers to be performed. ${ }^{[2]}$ (as explained in Chapter 3 and described below, Figure 4.2).

$J(V)$ measurements were performed by biasing the $\left(\mathrm{Ga}_{2} \mathrm{O}_{3}\right)$ EGaIn top-electrode and connecting the Au bottom-electrode to ground. As explained in Chapter 3, and as previously published, ${ }^{[2]}$ an in depth statistical analysis was carried out for all supramolecular junction measurements. Each averaged $|J|$ was calculated by plotting the $|J|$ measured at each voltage value (step size $0.10 \mathrm{~V}$, in both sweep directions, with total sweep being $\pm 2.0 \mathrm{~V}$ ) into histograms, giving in total 82 individual histograms. All histograms were plotted on a log scale, giving a normal distribution, allowing the data to be fit with Gaussian curves. This gave the average $|J|$ (the log mean) and the error (one-log standard deviation, $68 \%$ of the data is within one log standard deviation of the log-mean) for each voltage value, allowing the construction of the average $|J|$ vs. $V$ scan, with one single data point on the graph representing one Gaussian fit histogram. Figure 4.2 gives an example of how the averaged data points were calculated to obtain the average $|J|(V)$ scan with the error bars on the graph representing one log standard deviation from the log mean value, indicating between which $J$ values $68 \%$ of our total obtained $J$ measurements lie. Figure 4.3 shows an overlay semi log plot of the averaged (log mean) $|J|$ vs. $V$, for all EGaIn supramolecular junction structures (For clarity error bars are not shown, the individual semi-log plots of the averaged (log mean) $|J|$ vs. $V$ for all EGaIn supramolecular junction structures, including error bars can be found in the appendix (A.4.1))

Each individual $\left(\mathrm{Ga}_{2} \mathrm{O}_{3}\right)$ EGaIn junction was also characterized as either a 'working junction' (junction that gave 20 reproducible scans $\pm 2.0 \mathrm{~V}$ with the current measured being within $3 \sigma_{\log }$ of the $\mu_{\log }$ value), a 'short' (a junction that produced an immediate ohmic response, or a junction that suddenly produced an ohmic response during the 20 scans \pm 2.0 $\mathrm{V}$ ), or an 'unstable junction' (during the 20 scans $\pm 2.0 \mathrm{~V}$ the current measured varied greater than $3 \sigma_{\log }$ from the $\mu_{\log }$ value; Table 1). Considering all junction structures, working junctions were found with an average yield of $80-85 \%$ (Table 4.1 ), which is in accordance with other molecular structures measured in $\left(\mathrm{Ga}_{2} \mathrm{O}_{3}\right)$ EGaIn junctions. ${ }^{[2,13]}$ 
Table 4.1: Statistical overview of all $\left(\mathrm{Ga}_{2} \mathrm{O}_{3}\right)$ EGaIn junctions measured that contain generation one PPI dendrimers.

\begin{tabular}{|c|c|c|c|c|c|c|c|c|c|c|}
\hline $\begin{array}{l}\text { Molecular } \\
\text { Junction }^{\text {[a] }}\end{array}$ & $\begin{array}{l}\text { No. of } \\
\text { Substrates }{ }^{[\mathrm{b}]}\end{array}$ & $\begin{array}{l}\text { No. of } \\
\text { Junctions } \\
\text { Created }{ }^{[c]}\end{array}$ & $\begin{array}{l}\text { No. of } \\
\text { working } \\
\text { Junctions } \\
(\%)^{[d]}\end{array}$ & $\begin{array}{l}\text { No. of } \\
\text { Shorts }\end{array}$ & $\begin{array}{l}\text { No. of } \\
\text { Unstable } \\
\text { Junctions }{ }^{[f]}\end{array}$ & $\begin{array}{l}\text { Total } \\
\text { Scans } \\
\mid[\mid[\mid\end{array}$ & $R^{[\mathrm{h}]}$ & Error $(68 \%)^{[i]}$ & $\begin{array}{l}\text { No. of } \\
\text { interactions } \\
\text { with } \beta C D \\
\text { SAM }^{[j]}\end{array}$ & $\begin{array}{l}\text { Surface } \\
\text { Coverage } \\
\text { of } \beta C D \\
\text { SAM }(\%)^{[k]}\end{array}$ \\
\hline 4.1 "Fc" & 4 & 22 & $17(77)$ & 3 & 2 & 340 & 7.7 & $2.5-24$ & 2 & $\sim 89$ \\
\hline 4.2 "BFc" & 3 & 21 & $20(95)$ & 1 & 0 & 400 & $1.7 \times 10^{2}$ & $89-3.2 \times 10^{2}$ & 3 & -100 \\
\hline 4.3 "Ad" & 7 & 30 & $24(80)$ & 4 & 2 & 480 & 0.70 & $0.28-1.8$ & 2 & $>95$ \\
\hline 4.4 "-" & 7 & 30 & $25(83)$ & 4 & 1 & 500 & 1.0 & $0.33-3.0$ & - & - \\
\hline
\end{tabular}

[a] $4.1=\mathrm{Au}^{\mathrm{TS}}-\beta \mathrm{CDSAM} / \mathrm{G} 1-\mathrm{PPI}-(\mathrm{Fc})_{4} /\left(\mathrm{Ga}_{2} \mathrm{O}_{3}\right) \mathrm{EGaIn}, 4.2=\mathrm{Au}^{\mathrm{TS}}-\beta \mathrm{CDSAM} / \mathrm{G} 1-\mathrm{PPI}-(\mathrm{BFc})_{4} / /\left(\mathrm{Ga}_{2} \mathrm{O}_{3}\right) \mathrm{EGaIn}$, $4.3=\mathrm{Au}^{\mathrm{TS}}-\beta$ CDSAM/G1-PPI-(Ad) $)_{4} /\left(\mathrm{Ga}_{2} \mathrm{O}_{3}\right)$ EGaIn, $4.4=\mathrm{Au}^{\mathrm{TS}}-\beta$ CDSAM $/ /\left(\mathrm{Ga}_{2} \mathrm{O}_{3}\right)$ EGaIn. $[\mathrm{b}]=1 \mathrm{~cm} \times 1 \mathrm{~cm}$ $\mathrm{Au}^{\mathrm{TS}}$ surface on glass. $[\mathrm{c}]=\left(\mathrm{Ga}_{2} \mathrm{O}_{3}\right)$ EGaIn top electrode contacting the molecular monolayer immobilized on the $\mathrm{Au}^{\mathrm{TS}}$ surface. $[\mathrm{d}]=$ junctions that gave 20 reproducible scans $\pm 2.0 \mathrm{~V}$ with the current measured being within $3 \sigma_{\log }$ of the $\mu_{\log }$ value, (number of working junctions/number of junctions created) [e] = a junction that produced an immediate ohmic response, or a junction that produced an ohmic response during the 20 scans \pm $2.0 \mathrm{~V}$. [f] = during the 20 scans $\pm 2.0 \mathrm{~V}$ the current measured varied greater than $3 \sigma_{\log }$ from the $\mu_{\log }$ value. [g] $=$ a voltage sweep, $0.0 \mathrm{~V} \rightarrow+2.0 \mathrm{~V} \rightarrow 0.0 \mathrm{~V} \rightarrow-2.0 \mathrm{~V} \rightarrow 0.0 \mathrm{~V}$. [h] $=|J(-2.0 \mathrm{~V})| / J(+2.0 \mathrm{~V}) \mid .[\mathrm{i}]=$ one logstandard deviation, $68 \%$ of the data is within one $\log$ standard deviation of the $\log$-mean. $[\mathrm{j}]=$ amount of dendrimer terminal moieties that form host-guest interactions with the $\beta C D S A M$, out of a possible total of four. $[\mathrm{k}]=$ surface coverage $(\%)$ of the dendrimer adsorbed to the supramolecular platform. 

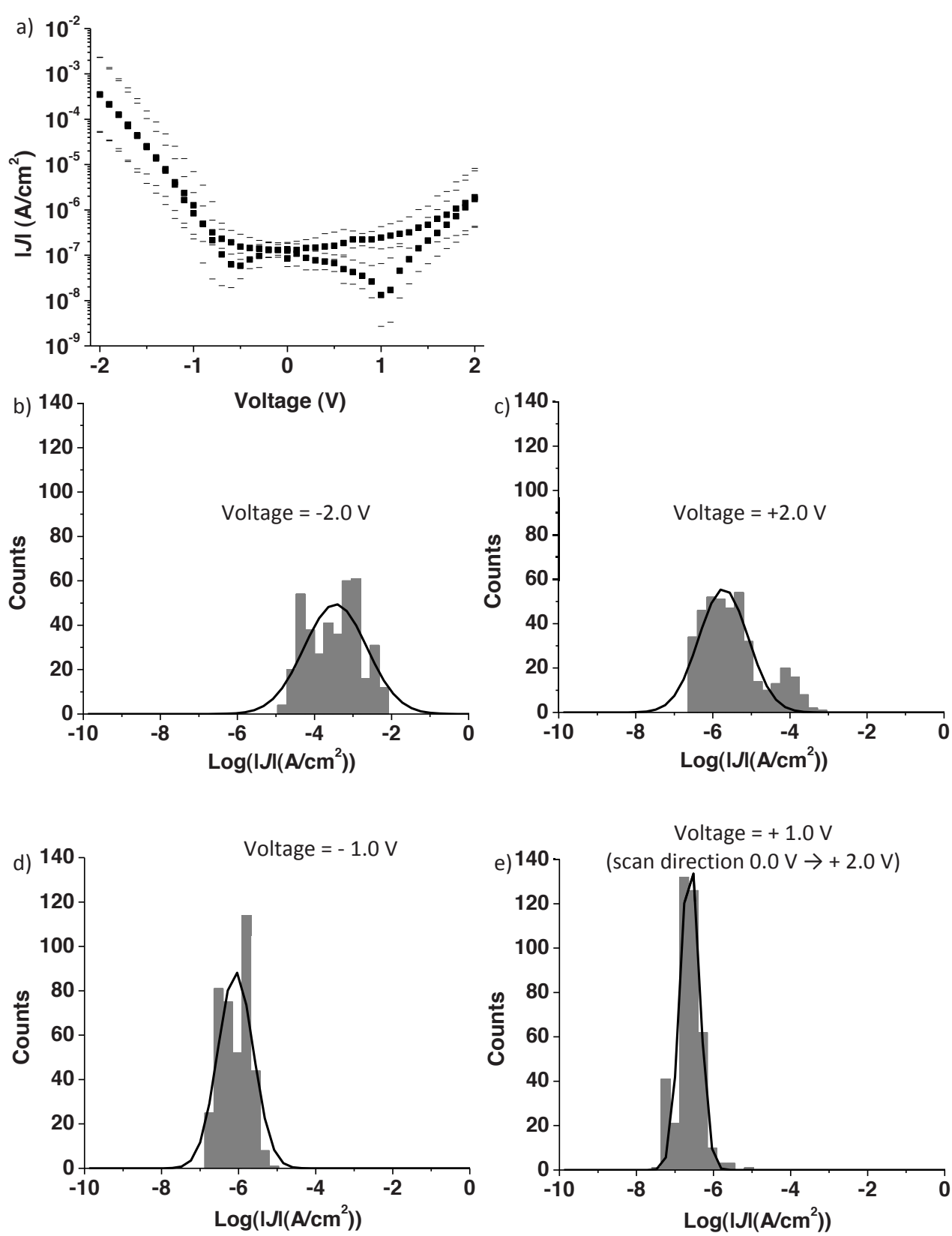

Figure 4.2: A semi-log plot of the averaged $|J|$ vs. $V$ for junction $\mathrm{Au}^{\mathrm{TS}}-\beta \mathrm{CDSAM} / \mathrm{G} 1-\mathrm{PPI}-$ $(\mathrm{BFc})_{4} / /\left(\mathrm{Ga}_{2} \mathrm{O}_{3}\right)$ EGaIn (4.2), (a), the statistical analysis for determining the averaged data point (log mean) at $2.0 \mathrm{~V}(\mathrm{~b}),+2.0 \mathrm{~V}(\mathrm{c}),-1.0 \mathrm{~V}(\mathrm{~d}),+1.0 \mathrm{~V}(\mathrm{e})$. Shorts cannot be seen on this scale as they have current densities of approximately $10^{\wedge}$ 


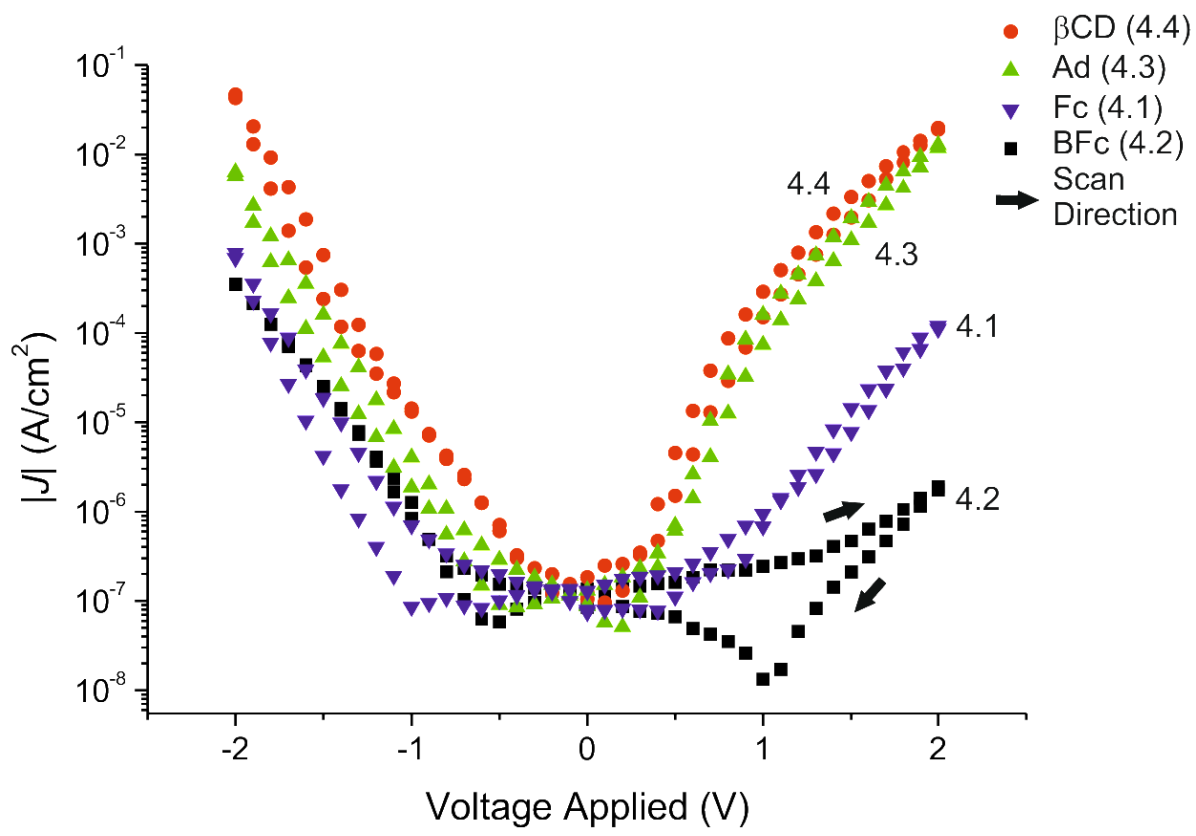

Figure 4.3: A semi-log plot of the averaged absolute current density vs. voltage $(|J|$ vs. $V)$ for junctions 4.1 4.4. The difference in the rectification ratio $(R)$ for each junction structure at $\pm 2.0 \mathrm{~V}$ is apparent. (For clarity error bars are not shown, the individual semi log plots of the averaged (log mean) $|J|$ vs. $V$ for all EGaIn supramolecular junction structures, including error bars can be found in the appendix)

\subsubsection{Supramolecular Rectification}

In Figure 4.3 the change of the $R$ value of all four junctions is clearly seen when comparing the relative difference in current densities at the outermost voltage points, that is, $-2.0 \mathrm{~V}$ and $+2.0 \mathrm{~V}$. The $R$ value [Eq. (4.1)] was calculated for each individual scan for each junction, and statistically analyzed in the same manner as the averaged $|J|(V)$ curves (Figure 4.4, also see Chapter 3 and experimental). ${ }^{[2]}$ Junctions 4.3 and 4.4 have small values of $R$ close to unity (Table 1). On the other hand, junctions 4.1 and 4.2 have significant values of $R, 7.7$ $\left(\sigma_{\log }=3.1\right)^{[19]}$ and $1.7 \times 10^{2}\left(\sigma_{\log }=1.9\right)$, respectively. The value of $R$ of $0.70\left(\sigma_{\log }=2.5\right)$ for junction 4.3 is less than unity because for these junctions, unlike the other junctions, the values of $J$ at a positive bias were larger than at a negative bias and we used for all junctions Eq (4.1) to calculate the values of $R$. It seems that neither the bare supramolecular platform 
nor the additional asymmetry created within the junction by adsorbing a PPI dendrimer on the supramolecular platform caused rectification. Thus, the significant values of $R$ in junctions 4.1 and 4.2, indicate that the $\mathrm{Fc}$ or $\mathrm{BFc}$ moieties are required for this supramolecular junction to act as a molecular rectifier.
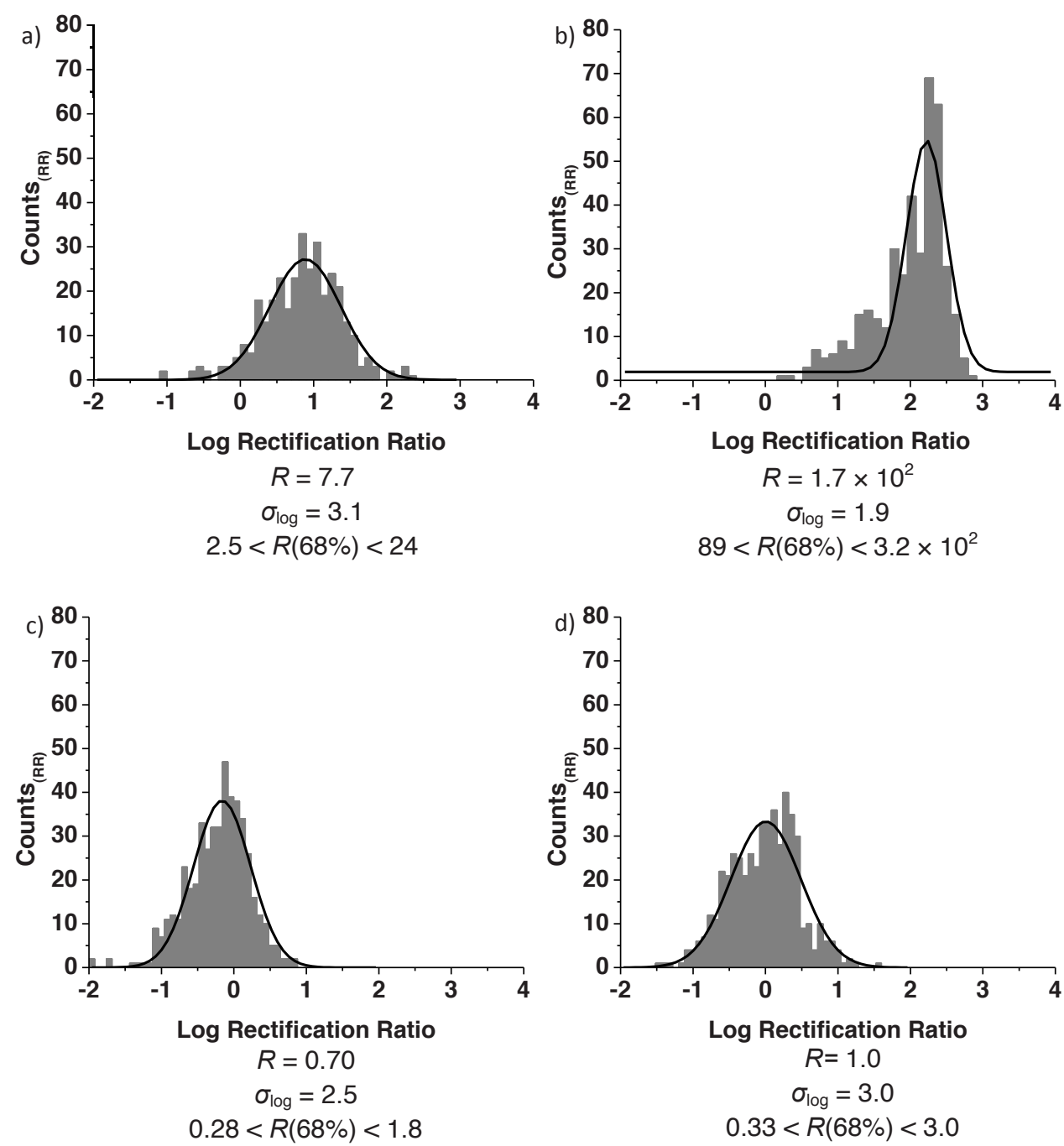

Figure 4.4: Histograms of $\log$ Rectification ratio $(R)$ for all molecular junction structures, $4.1=\mathrm{Au}^{\mathrm{TS}}$ $\beta C D S A M / G 1-P P I-(F c)_{4} / /\left(\mathrm{Ga}_{2} \mathrm{O}_{3}\right)$ EGaIn (a), $4.2=\mathrm{Au}^{\mathrm{TS}}-\beta C D S A M / G 1-P P I-(\mathrm{BFc})_{4} / /\left(\mathrm{Ga}_{2} \mathrm{O}_{3}\right)$ EGaIn (b), $4.3=$ $\mathrm{Au}^{\mathrm{TS}}-\beta C D S A M / G 1-P P I-(\mathrm{Ad})_{4} / /\left(\mathrm{Ga}_{2} \mathrm{O}_{3}\right)$ EGaIn (c), $4.4=\mathrm{Au}^{\mathrm{TS}}-\beta \operatorname{CDSAM} / /\left(\mathrm{Ga}_{2} \mathrm{O}_{3}\right) \mathrm{EGaIn}(\mathrm{d})$. All histograms were fitted with a Gaussian curve to obtain the $\log$ mean $(\mu l o g)$ and $\log$ standard deviation $\left(\sigma_{\log }\right)$ thus allowing for the calculation of $R(68 \%)$. 


\subsubsection{Mechanism of Charge Transport}

The Fc and BFc functionalized PPI dendrimers, are important for providing a low lying HOMO level, that is, a HOMO level close to that of the Fermi levels of the electrodes (as discussed in Chapter 2). The HOMO level for the G1-PPI-(Fc) $)_{4}$ and G1-PPI-(BFc) 4 dendrimers were estimated as $-5.1 \mathrm{eV}$ and $-5.0 \mathrm{eV}$ respectively, relative to vacuum, from cyclic voltammetry ${ }^{[14 f, 14 h]}$ using Eq (4.2), where $E_{\mathrm{NHE}, \text { abs }}=$ absolute potential energy of the normal hydrogen electrode $(-4.5 \mathrm{eV})$ and $\mathrm{E}_{1 / 2} \mathrm{NHE}=$ formal potential vs. normal hydrogen electrode (which is $0.466 \mathrm{eV}$ ).

$$
\mathrm{E}_{\mathrm{HOMO}}=\mathrm{E}_{\mathrm{NHE}, \mathrm{abs}}-\mathrm{eE}_{1 / 2, \mathrm{NHE}}
$$

These HOMO levels fulfill this requirement, as the Fermi level of $\mathrm{Au}$ and $\left(\mathrm{Ga}_{2} \mathrm{O}_{3}\right) \mathrm{EGaIn}$ is $\sim-5.1 \mathrm{eV}$ and $\sim-4.3 \mathrm{eV}$, respectively. We believe that junctions 4.1 and 4.2 rectify current by the mechanism proposed by the groups of Banger and co-workers, ${ }^{[20]}$ and Williams and co-workers, ${ }^{[21]}$ and more importantly experimentally shown and discussed by Whitesides and co-workers. $\left.{ }^{[2,}, 8,22\right]$ (discussed in Chapter 2). They proposed that an asymmetrically positioned molecular orbital, (either HOMO or LUMO) inside a tunneling junction, can rectify current. Whitesides and co-workers performed temperature dependant $J(V)$ measurements on a molecular junction similar to that reported here, $\mathrm{Ag}^{\mathrm{TS}}-$ $\mathrm{SC}_{11} \mathrm{Fc} / /\left(\mathrm{Ga}_{2} \mathrm{O}_{3}\right) \mathrm{EGaIn} .{ }^{[8]}$ They reported that tunneling (which is temperature independent) dominated the mechanism of charge transport at a positive bias and that hopping (which occurs when the HOMO level of the functional moiety overlaps with both the Fermi levels of the electrodes) dominated the mechanism of charge transport at a negative bias. ${ }^{[8]}$ As the Fc and BFc PPI dendrimers, in junctions 4.1 and 4.2, are placed spatially asymmetrically inside the junction, i.e., close to and coupled with the $\left(\mathrm{Ga}_{2} \mathrm{O}_{3}\right)$ EGaIn top-electrode and separated from the $\mathrm{Au}^{\mathrm{TS}}$ bottom-electrode by the $\beta \mathrm{CD}$ SAM, with HOMO levels that have energies close to that of the Fermi levels of the electrodes, they satisfy the criteria for a molecular rectifying junction and we believe that a mechanism of charge transport applies to our junction similar to that reported by Whitesides and co-workers ${ }^{[8]}$ (Figure 4.5).

Figure 4.5 displays the molecular energy diagram for our supramolecular tunneling junction containing the $\beta C D$ SAM + G1-PPI- $(\mathrm{BFc})_{4}$ dendrimer (junction 4.2 ) at a positive bias of $2.0 \mathrm{~V}$ (left) and a negative bias of $2.0 \mathrm{~V}$ (right). The PPI dendrimers form a van der Waals 
contact with the $\left(\mathrm{Ga}_{2} \mathrm{O}_{3}\right)$ EGaIn top-electrode, but are separated from the $\mathrm{Au}^{\mathrm{TS}}$ bottomelectrode by the $\beta C D$ SAM. The HOMO level of the BFc moiety is thus asymmetrically placed close to and coupled with the orbitals of the $\left(\mathrm{Ga}_{2} \mathrm{O}_{3}\right)$ EGaIn top-electrode. The estimated HOMO level of the BFc dendrimers (from $\mathrm{CV}$ ) is $-5.0 \mathrm{eV}$ which lies very close to the Fermi levels of the electrodes, $\sim-5.0 \mathrm{eV}$ for $\mathrm{Au}$ and $\sim-4.3 \mathrm{eV}$ for $\left(\mathrm{Ga}_{2} \mathrm{O}_{3}\right) \mathrm{EGaIn}$. Thus, the $\beta C D$ SAM may have a similar function as the $C_{11}$ alkyl chain in SAMs of $\mathrm{SC}_{11} \mathrm{Fc}$ and separates the $\mathrm{Fc}$ or BFc moieties from the bottom-electrode and, thus, provides asymmetry in the junctions, and the Fc and BFc moieties (regardless whether they interact or not with a $\mathrm{CD}$ moiety) of the PPI dendrimers, as in the case of the $\mathrm{SAMs}$ of $\mathrm{SC}_{11} \mathrm{Fc}$, provide a HOMO level that is in energy slightly off set with the Fermi levels of the electrodes.

When performing the experiments, the $\left(\mathrm{Ga}_{2} \mathrm{O}_{3}\right)$ EGaIn top-electrode was biased, and the $\mathrm{Au}^{\mathrm{TS}}$ bottom-electrode was connected to ground. As most of the potential drops across the $\beta C D$ SAM, the HOMO level of the BFc moiety follows the potential of the $\left(\mathrm{Ga}_{2} \mathrm{O}_{3}\right)$ EGaIn top-electrode. At a positive bias the Fermi level of the $\left(\mathrm{Ga}_{2} \mathrm{O}_{3}\right)$ EGaIn top-electrode decreases $(-6.3 \mathrm{eV})$ and therefore also does the HOMO level of the BFc $(-6.4 \mathrm{eV})$ (Figure 4.5 (right)). The HOMO level of the BFc moiety does not change by the same amount as the Fermi level of the $\left(\mathrm{Ga}_{2} \mathrm{O}_{3}\right)$ EGaIn top-electrode, because some of the applied potential will drop across the van der Waals interface. This leads to the BFc moiety not being able to participate in charge transport, as its HOMO level does not overlap with the Fermi levels of both electrodes, thus suggesting that tunneling is the dominant mechanism of charge transport at a positive bias. However, at a negative bias the Fermi level of the $\left(\mathrm{Ga}_{2} \mathrm{O}_{3}\right) \mathrm{EGaIn}$ top-electrode increases $(-2.3 \mathrm{eV})$ and therefore also does the HOMO level of the $\mathrm{BFc}$ $(-3.5 \mathrm{eV})$ (Figure 4.5 (left)). This leads to the BFc moiety being able to participate in charge transport, as its HOMO level does overlap with the Fermi levels of both electrodes, thus suggesting that hopping is a possible mechanism of charge transport at a negative bias. Therefore, the values of $J$ are larger at a negative bias than at a positive bias and rectification occurs as the BFc moiety can only participate in charge transport at a negative bias. 


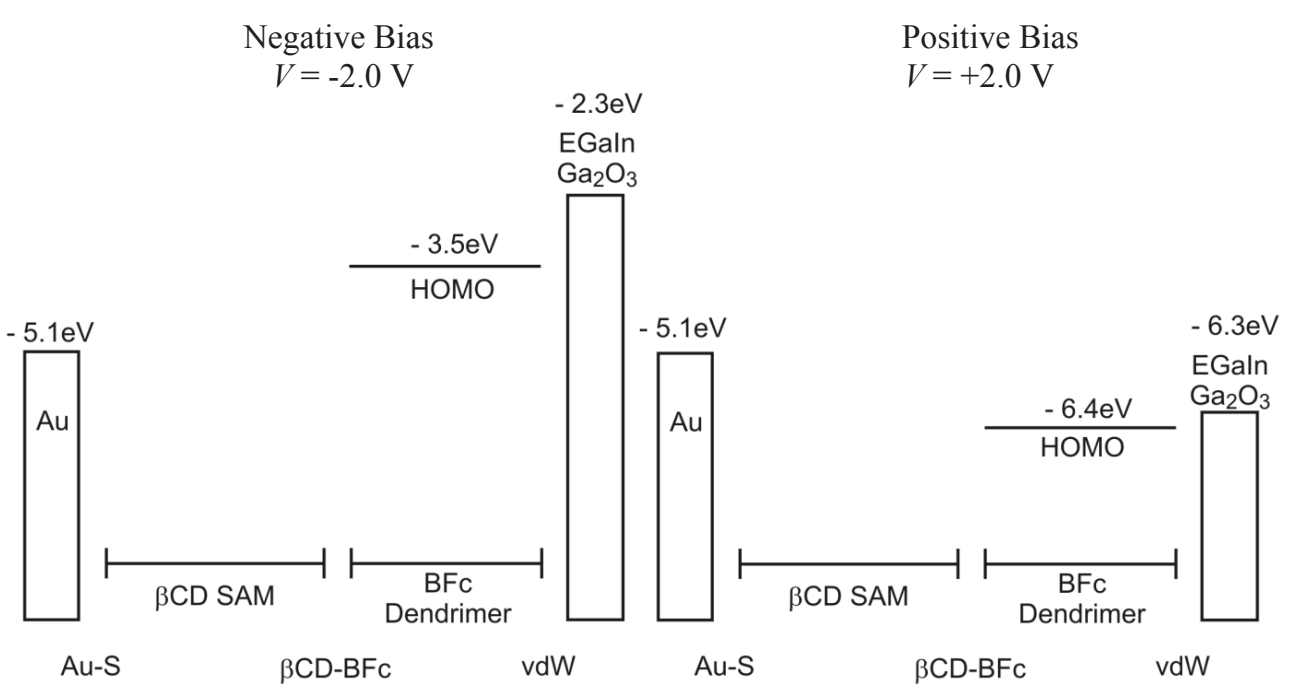

Figure 4.5: The proposed molecular energy diagram for $\mathrm{Au}^{\mathrm{TS}}-\beta \mathrm{CDSAM} / \mathrm{G} 1-\mathrm{PPI}-(\mathrm{BFc})_{4} / /\left(\mathrm{Ga}_{2} \mathrm{O}_{3}\right) \mathrm{EGaIn}$, (junction 4.2 ) at $-2.0 \mathrm{~V}$ (left) and $+2.0 \mathrm{~V}$ (right). $\mathrm{Au}-\mathrm{S}=$ the gold-thiolate interface, $\beta \mathrm{CD}-\mathrm{BFc}=$ the supramolecular host-guest interaction between the $\beta C D$ SAM and the BFc terminal group of the dendrimer, $\mathrm{vdW}=$ van der Waals interface. Total thickness of the molecular layer $=\sim 4.5 \mathrm{~nm}, \beta C D$ SAM $=\sim 2.5 \mathrm{~nm}$ (calculated from EIS) ${ }^{[14 b]}$ and G1-PPI-(BFc) $)_{4}=\sim 2 \mathrm{~nm}$ (estimated from molecular dynamic modeling). ${ }^{[15]}$

The large difference of two orders of magnitude in the values of $R$ measured for the $\mathrm{Fc}$ and $\mathrm{BFc}$ functionalized systems is most likely caused by the fact that the G1-PPI-(Fc $)_{4}$ forms a discontinuous monolayer on the $\beta C D$ SAM, while the G1-PPI-(BFc $)_{4}$ forms a continuous monolayer (Table 4.1). ${ }^{[13 \mathrm{e}-\mathrm{h}]}$ Thus, contacting the sub-monolayer of G1-PPI- $(\mathrm{Fc})_{4}$ on the $\beta C D$ SAM results in junctions in which the $\left(\mathrm{Ga}_{2} \mathrm{O}_{3}\right)$ EGaIn top-electrodes form contacts with the G1-PPI-(Fc) $)_{4}$ dendrimers (approx. 90\% per unit area) and with the $\beta C D$ SAM (approx. $10 \%$ per unit area). The areas with $\left(\mathrm{Ga}_{2} \mathrm{O}_{3}\right) \mathrm{EGaIn}$ in contact with the $\beta \mathrm{CD}$ SAM can be referred to as "thin area defects". ${ }^{[7]}$ The tunneling current $J\left(\mathrm{~A} / \mathrm{cm}^{2}\right)$ decays exponentially with the distance between the two electrodes $d(\AA)$, as approximated by a simple form of the Simmons equation, $J=J_{0} \mathrm{e}^{-\beta \mathrm{d}}$, (where $J_{0}\left(\mathrm{~A} / \mathrm{cm}^{2}\right)$ is the current density flowing through the electrode-SAM interfaces in the hypothetical case of $d=0 \AA$, and $\beta\left(\AA^{-1}\right)$ is the decay constant). Consequently, these thin area defects dominate the measured tunneling current, ${ }^{[7]}$ which, in turn, decreases $R$ (see Figure 4.3). Unlike the G1-PPI- $(\mathrm{Fc})_{4}$ dendrimer, the G1PPI-(BFc $)_{4}$ dendrimer is able to bind to the supramolecular platform with three out of its 
four BFc terminal moieties, ${ }^{[14 \mathrm{~h}]}$ as the BFc moieties (which are also larger than Fc moieties) are connected to the dendritic core with longer tethers than those used to connect the $\mathrm{Fc}$ moieties. This binding results in a densely packed monolayer on the platform, which, in turn, minimizes the formation of direct contact of the top-electrode with the $\beta C D$ SAM. Consequently, these junctions are dominated by the PPI dendrimers within the molecular junctions and thus rectify more.

\subsubsection{Current Density and Hysteresis}

Interestingly, the differences in the average values of $J$ or the hysteresis of the supramolecular junctions are not understood. The difference in the average $J$ of all the supramolecular junction structures does produce an unclear trend. The average values for junction 4.4 are, as expected, higher than those observed for junctions 4.1 and 4.2. The values of $J$ for junctions 4.3 and 4.4 are, however, similar. The values of $J$ for 4.3 would be expected to be much lower than 4.4 because junction 4.3 is thicker due to the presence of the monolayer of the PPI dendrimer which, in turn, would result in lower values of $J$. Table 1 shows that the Ad PPI dendrimer forms a monolayer with a surface coverage of $~ 95 \%$. Thus, also in these junctions the liquid metal top-contact may form direct contacts with the $\beta C D$ SAM and increase the measured value of $J$. In addition, the log-standard deviations for these junctions are larger than for the other junctions which are probably the result of other variations between junctions that could affect $J$. In this chapter rectification was used to investigate charge transport, which has the advantage of using, within the same tunneling junction, the current measured at the forward bias as the reference for the current measured at the reverse bias; Eq (4.1). This minimizes many of the uncertainties and complexities, such as contact resistances and contact areas, which are associated with comparing values of $J$ obtained from different tunneling junctions. Even the most carefully prepared SAM-based tunneling junctions can exhibit larger variations in $J$ (see histograms of $J$, Figure 4.2b-e), but this will not effect the value of $R$. Thus, despite the error in the values of $J$, the trend observed for the values of $R$ holds. As the hysteresis is more prominent in junctions 4.1 and 4.2, we speculate that the hysteresis could be due to the oxidation/reduction of the $\mathrm{Fc}$ moieties present in these junctions. However, the $J$ measured at the biases where the hysteresis occurs is small $\left(\sim 10^{-8}-10^{-7} \mathrm{~A} / \mathrm{cm}^{2}\right)$. As the data is presented on a semi-log plot, with the absolute values of $|J|$ on a logarithmic scale, these small currents are exaggerated. However, in Chapters 5 and 6, the concept of using a supramolecular platform in tunneling 
junctions allows us to systematically vary the type of functional dendrimer in the junction and investigate these unclear phenomena, rectification and the mechanism of charge transport in more detail.

\subsection{Conclusion}

In summary, first, a method is presented to fabricate well-defined tunneling junctions. This method makes it possible to conduct physical-organic studies by only altering the end group functionality of dendrimers anchored on a supramolecular platform while keeping other possible structural changes to a minimum. Second, the stability of the junctions permits the accumulation of statistically large amounts of data, which makes a statistical analysis possible to account for defects in the junctions. Third, this physical- organic study shows that the rectification is induced by the $\mathrm{Fc}$ and $\mathrm{BFc}$ moieties, positioned asymmetrically inside the junction, and is of molecular origin and not due to any other asymmetries in the junction. Finally, the rectification ratio is controlled by changing the end group functionality of the dendrimer. In Chapters 5 and 6 of this thesis these supramolecular surface interactions are further exploited in $\left(\mathrm{Ga}_{2} \mathrm{O}_{3}\right)$ EGaIn tunneling junctions by varying the dendrimer generation and core type. This allowed for the rectification within the supramolecular junction to be further controlled and contributed to the fundamental understanding of charge transport in molecular tunneling junctions.

\subsection{Experimental Details}

Materials: Preparation of heptathioether-functionalized $\beta$-cyclodextrin ${ }^{[14 a]}$ and G1-PPI$(\mathrm{Ad})_{4},{ }^{[23]} \mathrm{G} 1-\mathrm{PPI}-(\mathrm{Fc})_{4}{ }^{[24]}$ and G1-PPI-(BFc) ${ }_{4}{ }^{[14 \mathrm{~h}]}$ dendrimers was described previously. All compounds were characterized with Nuclear Magnetic Resonance Spectroscopy $\left({ }^{1} \mathrm{H}\right.$ NMR) and Mass Spectrometry (MALDI ToF and ESI-MS), with all compounds yielding similar results to what has been previously published. Eutectic Gallium Indium was used as purchased from Aldrich.

\section{Method:}

\section{SAM Formation}

Ultra flat substrates of thin films of gold metal on a polymer supported by a glass slide, were obtained by delaminating an evaporated film of gold from a $\mathrm{Si} / \mathrm{SiO}_{2}$ template (the 'mechanical template-stripping' procedure (TS)) as previously described in detail. ${ }^{[17]} \beta \mathrm{CD}$ 
SAMs were prepared by immersing freshly cleaved ultra-flat gold substrates into a $0.1-1$ $\mathrm{mM}$ solution of $\beta \mathrm{CD}$ dissolved in ethanol for 16 hours at $60^{\circ} \mathrm{C}$. The substrates were removed and placed in a vial of fresh warm ethanol with the vial being gently agitated for one to two minutes. After removal they were further rinsed with ethanol (room temperature) and MilliQ water, and then dried under a stream of dry nitrogen. As previously published, Electrochemical Impedance Spectroscopy was used to characterize control $\beta C D$ SAMs and yielded similar results.

\section{Dendrimer Absorption}

Substrates that were to contain PPI dendrimers were immersed in an aqueous solution of the corresponding dendrimer- $\beta C D$ assembly; G1-PPI-(Ad) $)_{4}$ and G1-PPI-(Fc) $)_{4}$ dendrimer, 2-3 $\mathrm{mM}$ in $\mathrm{Ad} / \mathrm{Fc}$ concentration in the presence of $2-3 \mathrm{mM}$ of $\beta \mathrm{CD}$ at $\mathrm{pH}=2$ for at least $1 \mathrm{hr}$, G1-PPI-(BFc) $)_{4}$ dendrimer, $1-2 \mathrm{mM}$ in BFc functionality in the presence of $10 \mathrm{mM} \beta \mathrm{CD}$ at $\mathrm{pH}=2$, for at least 2.5 hours. ${ }^{[14 f, 14 \mathrm{~h}]}$ Subsequently the samples were rinsed with MilliQ water and dried under a stream of dry nitrogen. As previously published, Surface Plasmon Resonance and Cyclic Voltammetry were used to characterize the control $\beta C D$ SAM + dendrimer assemblies and yielded similar results.

\section{Calculation of Rectification Ratio}

The rectification ratio which is defined as $R=|J(-2.0 \mathrm{~V})| /|J(+2.0 \mathrm{~V})|$ was analyzed in the same fashion as the $J$ measurements for each molecular junction and as previously published. ${ }^{[2]}$ The $R$ for each individual scan was calculated at $\pm 2.0 \mathrm{~V}$ for each supramolecular junction structure and plotted into histograms. Fitting the histograms with single Gaussian functions gave the log-mean value (average) $R$ and the log standard deviation (with all errors stated representing one log-standard deviation, 68\% of the distribution of the data is within one log-standard deviation of the log-mean, as shown in Figure 4.4 this chapter and discussed in Chapter 3).

\subsection{References}

[1] $68 \%$ of the distribution of $R$ lies within one log standard deviation ( $\sigma_{\log }$ ) of the log mean $\left(\mu_{\mathrm{log}}\right)$, for example if $\mu_{\mathrm{log}}=170$ and $\sigma_{\mathrm{log}}=1.6$ than $68 \%$ of the distribution of $R$ lies within the range of $106(170 / 1.6)$ and $272(170 * 1.6)$.

[2] C. A. Nijhuis, W. F. Reus, G. M. Whitesides, J. Am. Chem. Soc. 2009, 131, 17814-17827. 
[3] a)R. G. Endres, D. L. Cox, R. R. P. Singh, Rev. Mod. Phys. 2004, 76, 195-214; b)M. Gratzel, J Photochem. Photobiol. C-Photochem Rev. 2003, 4, 145-153; c)R. L. McCreery, Chem. Mater. 2004, 16, 4477-4496; d)J. C. Scott, L. D. Bozano, Adv. Mater. 2007, 19, 1452-1463; e)D. M. Adams, L. Brus, C. E. D. Chidsey, S. Creager, C. Creutz, C. R. Kagan, P. V. Kamat, M. Lieberman, S. Lindsay, R. A. Marcus, R. M. Metzger, M. E. Michel-Beyerle, J. R. Miller, M. D. Newton, D. R. Rolison, O. Sankey, K. S. Schanze, J. Yardley, X. Y. Zhu, J. Phys. Chem. B 2003, 107, 6668-6697.

[4] a)T.-W. Kim, G. Wang, H. Lee, T. Lee, Nanotechnology 2007, 18, 315204; b)M. D. Austin, S. Y. Chou, Nano Lett. 2003, 3, 1687-1690; c)G. S. Bang, H. Chang, J. R. Koo, T. Lee, R. C. Advincula, H. Lee, Small 2008, 4, 1399-1405.

[5] a)G. L. Fisher, A. V. Walker, A. E. Hooper, T. B. Tighe, K. B. Bahnck, H. T. Skriba, M. D. Reinard, B. C. Haynie, R. L. Opila, N. Winograd, D. L. Allara, J. Am. Chem. Soc. 2002, 124, 5528-5541; b)A. V. Walker, T. B. Tighe, O. M. Cabarcos, M. D. Reinard, B. C. Haynie, S. Uppili, N. Winograd, D. L. Allara, J. Am. Chem. Soc. 2004, 126, 3954-3963; c)J. M. Beebe, J. G. Kushmerick, Appl. Phys. Lett. 2007, 90; d)C. N. Lau, D. R. Stewart, R. S. Williams, M. Bockrath, Nano Lett. 2004, 4, 569-572.

[6] J. C. Love, L. A. Estroff, J. K. Kriebel, R. G. Nuzzo, G. M. Whitesides, Chem. Rev. 2005, 105, 1103-1169.

[7] E. A. Weiss, R. C. Chiechi, G. K. Kaufman, J. K. Kriebel, Z. Li, M. Duati, M. A. Rampi, G. M. Whitesides, J. Am. Chem. Soc. 2007, 129, 4336-4349.

[8] C. A. Nijhuis, W. F. Reus, J. R. Barber, M. D. Dickey, G. M. Whitesides, Nano Lett. 2010, 10, 3611-3619.

[9] A. Aviram, M. A. Ratner, Chem. Phys. Lett. 1974, 29, 277-283.

[10] a)M. L. Chabinyc, X. Chen, R. E. Holmlin, H. Jacobs, H. Skulason, C. D. Frisbie, V. Mujica, M. A. Ratner, M. A. Rampi, G. M. Whitesides, J. Am. Chem. Soc. 2002, 124, 11730-11736; b)M.K. Ng, D.-C. Lee, L. Yu, J. Am. Chem. Soc. 2002, 124, 11862-11863; c)R. McCreery, J. Dieringer, A. O. Solak, B. Snyder, A. M. Nowak, W. R. McGovern, S. DuVall, J. Am. Chem. Soc. 2003, 125, 10748-10758; d)T. Böhme, C. D. Simpson, K. Müllen, J. P. Rabe, Chem. Eur. J. 2007, 13, 7349-7357; e)A.-S. Hallbäck, B. Poelsema, H. J. W. Zandvliet, Solid State Commun. 2007, 141, 645-648; f)I. Dies-Perez, J. Hihath, Y. Lee, L. Yu, L. Adamska, M. A. Kozhushner, I. I. Oleynik, N. Tao, Nature Chem. 2009, 1, 635-641; g)M. Elbing, R. Ochs, M. Koentopp, M. Fischer, C. von Hanisch, F. Weigend, F. Evers, H. B. Weber, M. Mayor, Proc. Natl. Acad. Sci. USA 2005, 102, 8815-8820.

[11] a)R. M. Metzger, Anal. Chim. Acta 2006, 568, 146-155; b)G. J. Ashwell, B. Urasinska, W. D. Tyrrell, Phys. Chem. Chem. Phys. 2006, 8, 3314-3319. 
[12] a)S. Lenfant, D. Guerin, F. Tran Van, C. Chevrot, S. Palacin, J. P. Bourgoin, O. Bouloussa, F. Rondelez, D. Vuillaume, J. Phys. Chem. B 2006, 110, 13947-13958; b)C. Zhou, M. R. Deshpande, M. A. Reed, L. Jones Ii, J. M. Tour, Appl. Phys. Lett. 1997, 71, 611-613; c)S. Lenfant, C. Krzeminski, C. Delerue, G. Allan, D. Vuillaume, Nano Lett. 2003, 3, 741-746.

[13] R. C. Chiechi, E. A. Weiss, M. D. Dickey, G. M. Whitesides, Angew. Chem. Int. Ed. 2008, 47, 142-144.

[14] a)M. W. J. Beulen, J. Bugler, B. Lammerink, F. A. J. Geurts, E. M. E. F. Biemond, K. G. C. van Leerdam, F. C. J. M. van Veggel, J. F. J. Engbersen, D. N. Reinhoudt, Langmuir 1998, 14, 6424-6429; b)M. W. J. Beulen, J. Bügler, M. R. de Jong, B. Lammerink, J. Huskens, H. Schönherr, G. J. Vancso, B. A. Boukamp, H. Wieder, A. Offenhäuser, W. Knol, F. C. J. M. van Veggel, D. N. Reinhoudt, Chem. Eur. J. 2000, 6, 1176-1183; c)J. Huskens, M. A. Deij, D. N. Reinhoudt, Angew. Chem. Int. Ed. 2002, 41, 4467-4471; d)M. J. W. Ludden, D. N. Reinhoudt, J. Huskens, Chem. Soc. Rev 2006, 35, 1122-1134; e)C. A. Nijhuis, J. Huskens, D. N. Reinhoudt, J. Am. Chem. Soc. 2004, 126, 12266-12267; f)C. A. Nijhuis, F. Yu, W. Knoll, J. Huskens, D. N. Reinhoudt, Langmuir 2005, 21, 7866-7876; g)C. A. Nijhuis, B. A. Boukamp, B. J. Ravoo, J. Huskens, D. N. Reinhoudt, J. Phys. Chem. C 2007, 111, 9799-9810; h)C. A. Nijhuis, K. A. Dolatowska, B. J. Ravoo, J. Huskens, D. N. Reinhoudt, Chem. Eur. J. 2007, 13, 69-80; i)C. A. Nijhuis, N. Oncel, J. Huskens, H. J. W. Zandvliet, B. J. Ravoo, B. Poelsema, D. N. Reinhoudt, Small 2006, 2, 1422-1426; j)C. M. Bruinink, C. A. Nijhuis, M. Peter, B. Dordi, O. Crespo-Biel, T. Auletta, A. Mulder, H. Schonherr, G. J. Vancso, J. Huskens, D. N. Reinhoudt, Chem. Eur. J. 2005, 11, 3988-3996; k)C. A. Nijhuis, J. K. Sinha, G. Wittstock, J. Huskens, B. J. Ravoo, D. N. Reinhoudt, Langmuir 2006, 22, 9770-9775.

[15] D. Thompson, Langmuir 2007, 23, 8441-8451.

[16] a)J. Szejtli, J. Mater. Chem. 1997, 7, 575-587; b)We expect that the supramolecular structures inside the tunnel junction resemble the supramolecular structures deduced from solution based studies.

[17] E. A. Weiss, G. K. Kaufman, J. K. Kriebel, Z. Li, R. Schalek, G. M. Whitesides, Langmuir 2007, 23, 9686-9694.

[18] At this moment we cannot deduce the different roles that CD complexed and free standing FC and BFC moities play in the molecular rectification, and therefore have only presented a simple mechanism hypothesis, where all functional moities are asymmetrically positioned with the junction.

[19] $\mu_{\log }=8$ and $\sigma_{\log }=3.1$, thus $68 \%$ of the distribution of $R$ is within the range 3 to $25,8 / 3.1$ and $8 * 3.1$.

[20] R. Liu, S. H. Ke, W. T. Yang, H. U. Baranger, J. Chem. Phys. 2006, 124, 024718. 
[21] P. E. Kornilovitch, A. M. Bratkovsky, R. S. Williams, Phys. Rev. B 2002, 66, 165436.

[22] C. A. Nijhuis, W. F. Reus, G. M. Whitesides, J. Am. Chem. Soc. 2010, 132, 18386-18401.

[23] M. Baars, A. J. Karlsson, V. Sorokin, B. F. W. de Waal, E. W. Meijer, Angew. Chem. Int. Ed. 2000, 39, 4262-4265.

[24] I. Cuadrado, M. Moran, C. M. Casado, B. Alonso, F. Lobete, B. Garcia, M. Ibisate, J. Losada, Organometallics 1996, 15, 5278-5280. 


\section{Appendix}

\section{Section A4.1:}

The averaged (log mean) $|J|$ vs. $V$ measurements for all EGaIn supramolecular junction structures, including error bars (error bars represent one-log standard deviation from the log mean, $68 \%$ of the data is within one-log standard deviation of the log-mean).
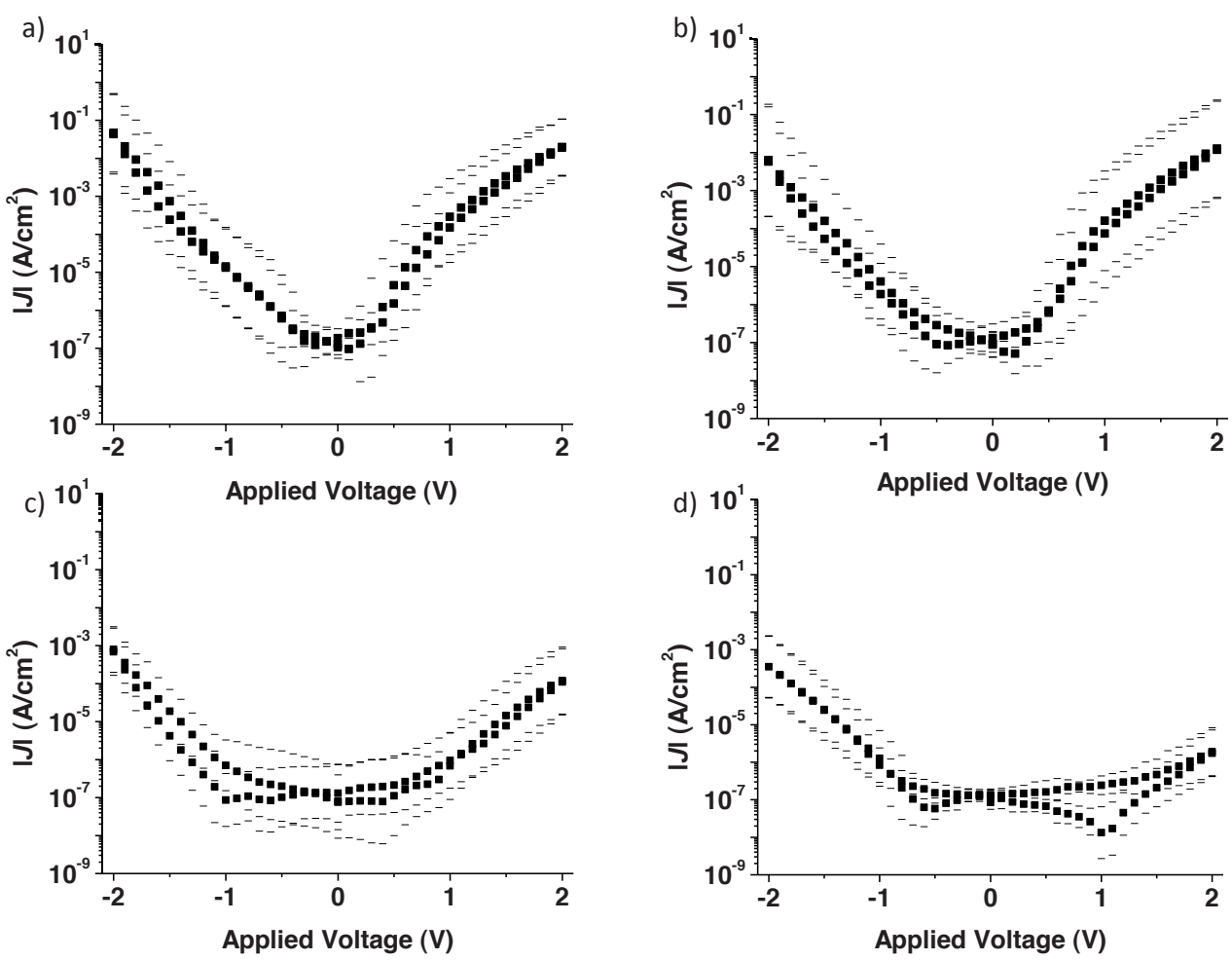

Figure A.4.1: A semi-log plot of the averaged $|J|$ vs. $V$ for junction $\mathrm{Au}^{\mathrm{TS}}-\beta \mathrm{CDSAM} / /\left(\mathrm{Ga}_{2} \mathrm{O}_{3}\right) \mathrm{EGaIn}$ (4.4) (a), junction $\quad \mathrm{Au}^{\mathrm{TS}}-\beta \mathrm{CDSAM} / \mathrm{G} 1-\mathrm{PPI}-(\mathrm{Ad})_{4} / /\left(\mathrm{Ga}_{2} \mathrm{O}_{3}\right) \mathrm{EGaIn} \quad$ (4.3) (b), junction $\mathrm{Au}^{\mathrm{TS}}-\beta C D S A M / G 1-P P I-$ $(\mathrm{Fc})_{4} / /\left(\mathrm{Ga}_{2} \mathrm{O}_{3}\right)$ EGaIn (4.1) (c), junction $\mathrm{Au}^{\mathrm{TS}}$ - $\beta$ CDSAM/G1-PPI-(BFc) $)_{4} /\left(\mathrm{Ga}_{2} \mathrm{O}_{3}\right)$ EGaIn (4.2) (d). 


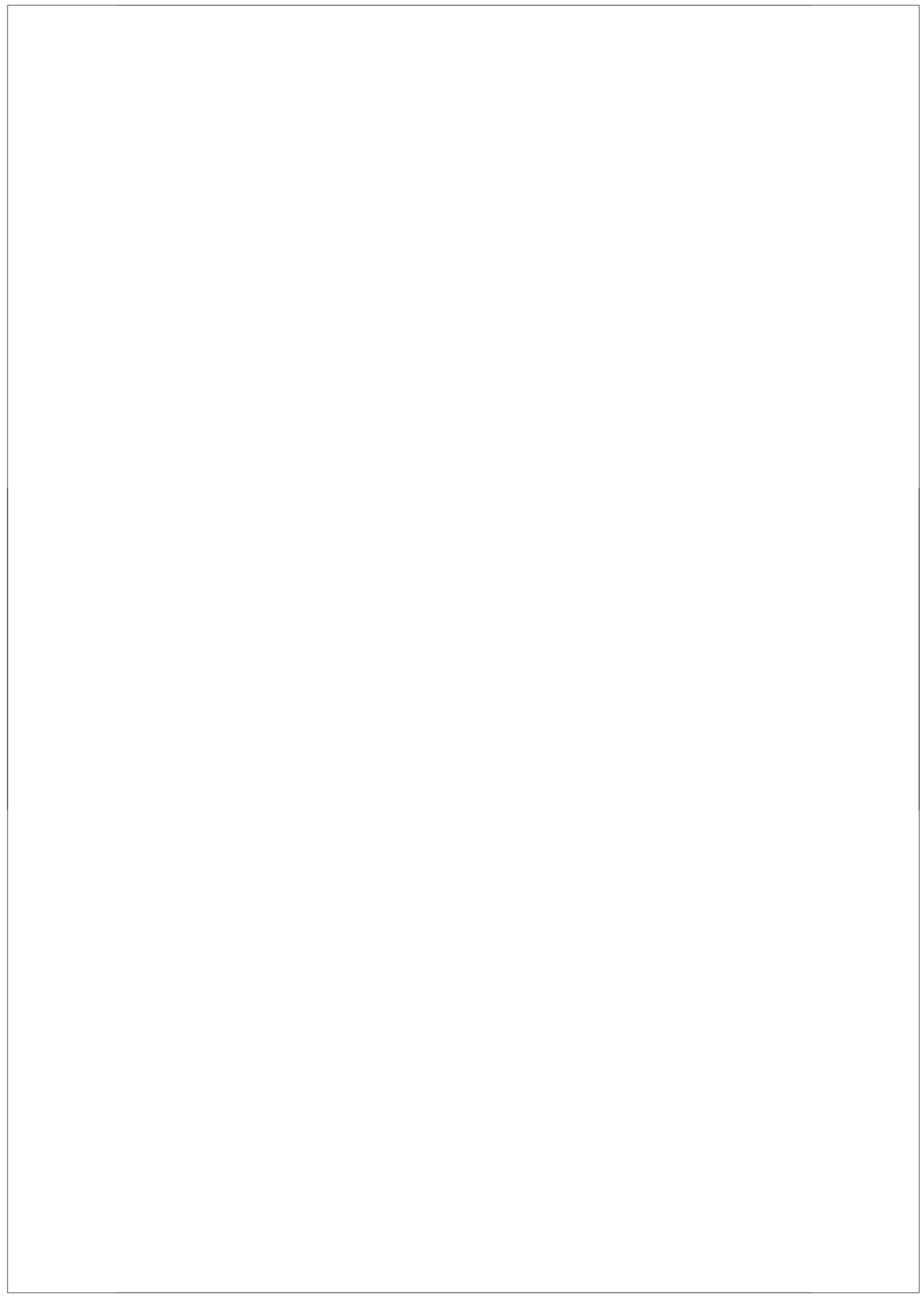




\section{Control Over Rectification in} Supramolecular Tunneling Junctions: Poly(amido amine)

\section{Dendrimers}

In the previous Chapter the ability to control rectification in supramolecular tunneling junctions by changing the terminal functional moiety of the generation one poly(propylene) imine dendrimer (G1-PPI) absorbed to the supramolecular platform was shown. Dendrimers containing ferrocene $(\mathrm{Fc})$ and bi-ferrocene $(\mathrm{BFc})$ terminal moieties were found to rectify currents. This gave two hypotheses. Hypothesis 1, that rectification occurs due to the Fc functional groups providing a low lying HOMO level with energies close to that of the Fermi levels of the electrodes, whilst being asymmetrically placed within the tunneling junction and close to and coupled with the top $\left(\mathrm{Ga}_{2} \mathrm{O}_{3}\right)$ EGaIn electrode, and hypothesis 2, the reason for the difference of two orders of magnitude in the values of $R$ measured for the $\mathrm{Fc}$ and $\mathrm{BFc}$ functionalized dendrimers was caused by the fact that the G1-PPI-(Fc) $)_{4}$ formed a discontinuous monolayer on the supramolecular platform causing 'thin area defects', whereas G1-PPI-(BFc) $)_{4}$ formed a continuous monolayer, thus minimizing the formation of thin area defects. In this Chapter these hypotheses are investigated in supramolecular tunneling junctions by: (1) changing the position of the terminal functional moiety (hence, the position of the HOMO level) within the junction and (2) changing the packing density of the dendrimer on the surface. This is achieved by changing the core of the dendrimer and the distance between the terminal function moieties by using a generation zero poly(amido amine) ferrocene functionalized dendrimer (G0-PAMAM- $\left.(\mathrm{Fc})_{4}\right)$ and a generation zero poly(amido amine) ethylene glycol linker-ferrocene functionalized dendrimer (G0PAMAM-((EG) $\left.\left.)_{3}-\mathrm{Fc}\right)_{4}\right)$. 


\subsection{Introduction}

In 1974 Aviram and Ratner proposed that molecules could function as diodes. ${ }^{[1]}$ Since then a variety of molecular assemblies, a majority of which are self-assembled-monolayers (SAMs), have been investigated in different types of molecular tunneling junctions. ${ }^{[2]}$ Many of these studies have claimed molecular architecture that rectify currents and thus act as molecular diodes. ${ }^{[3]}$ However, proving that the rectification is molecular in origin and determining the mechanism of charge transport within the junction is not trivial. This is primarily due to three reasons: First, studying the mechanism of charge transport as a function of molecular structure is difficult due to poorly defined structures of the SAMs. ${ }^{[3 \mathrm{k}, 4]}$ Second, physical-organic studies with statistically large numbers of data have rarely been performed $^{[2 \mathrm{c}, 2 \mathrm{f}, 3 \mathrm{~d}]}$ due to the instability, low yield and low reproducibility of the molecular junctions obtained by fabrication techniques based on the evaporation of a metal contacts directly on to the SAM. ${ }^{[2 \mathrm{f}]}$ Finally, molecular junctions that have their bottom and top electrodes fabricated from different materials or those that may have other non-organic asymmetries (for example an ill-defined a metal oxide layer), can have the ability to rectify current without the organic component having any structural asymmetry, which in turn complicates the interpretation of experimental data. ${ }^{[3 \mathrm{~h}, 5]}$

One approach to address the difficulty to contact SAMs electrically resulting in stable, reproducible, molecular tunneling junctions in high yields that allow for the accumulation of statistically large numbers of data, is to use the eutectic alloy of gallium and indium $\left(\left(\mathrm{Ga}_{2} \mathrm{O}_{3}\right)\right.$ EGaIn $)$ technique. ${ }^{[2 \mathrm{e}, 3 \mathrm{~b}, 3 \mathrm{~d}]}$ This technique allowed for very thorough investigations of the mechanisms of charge transport across SAMs of ferrocene functionalized alkanethiolates, on template stripped silver $\left(\mathrm{Ag}^{\mathrm{TS}}\right)$, with a $\left(\mathrm{Ga}_{2} \mathrm{O}_{3}\right)$ EGaIn top electrode. ${ }^{\text {[3a-d] }}$ The ferrocene $(\mathrm{Fc})$ functionalized n-alkanethiolates were found to rectify currents in DC measurements with $R=1.0 \times 10^{2}\left(\sigma_{\log }=3.0\right)$ at $\pm 1.0 \mathrm{~V}$ (where $\left.R=|J(-\mathrm{V})| /|J(+\mathrm{V})|\right)$, ${ }^{[3 \mathrm{~d}]}$ and act as the functional element of a half-wave rectifier (a simple circuit that converts or rectifies an alternating current (AC) to a direct current (DC). ${ }^{[3 \mathrm{a}]}$ Additionally by performing temperature dependent measurements ${ }^{[3 \mathrm{c}]}$ and systematical variations of the lengths and positions of the "insulating" and "conductive" molecular components within the Fc functionalized alkanethiolates, ${ }^{[3 b]}$ it was possible to determine the mechanism of rectification for these molecular tunneling junctions, and critically compare and contrast the results 
obtained to the theory of molecular rectification proposed by Williams and co-workers, ${ }^{[6]}$ Baranger and co-workers, ${ }^{[7]}$ and Ford and co-workers. ${ }^{[8]}$

Chapter 4 reports the ability to control rectification in well-defined supramolecular tunneling junctions. ${ }^{[9]}$ Monolayers of a variety of generation one poly(propylene) imine (G1-PPI) dendrimers on a $\beta$-cyclodextrin self-assembled monolayer ( $\beta C D$ SAM) on template stripped $\mathrm{Au}\left(\mathrm{Au}^{\mathrm{TS}}\right)$, were exploited in $\left(\mathrm{Ga}_{2} \mathrm{O}_{3}\right)$ EGaIn tunneling junctions. Control over the rectification $(R)$, (where $R=|J(-2.0 \mathrm{~V})| /|J(+2.0 \mathrm{~V})|$; [Eq. (4.1)]) in these junctions was achieved by varying the terminal functionality of the dendrimer from adamantyl $\left(\mathrm{Ad}, R=0.7, \sigma_{\log }=2.5\right)$ to ferrocene $\left(\mathrm{Fc}, R=7.7, \sigma_{\log }=3.1\right)$ and bi-ferrocene $(\mathrm{BFc}, R=1.7$ $\left.\times 10^{2}, \sigma_{\log }=1.9\right)$, with the bare $\beta$ CD SAM being measured as a control $\left(R=1.0, \sigma_{\log }=3\right)$. These results left two testable hypotheses.

1) Junctions containing $\mathrm{Fc}$ and $\mathrm{BFc}$ functionalized dendrimers rectified currents by the same mechanism proposed by Williams and co-workers ${ }^{[6]}$ and Baranger and co-workers, ${ }^{[7]}$ and experimentally shown by Whitesides and co-workers. ${ }^{[3 \mathrm{~b}-\mathrm{d}]}$ That is, that the Fc functional groups provided a low lying HOMO level with energies close to that of the Fermi levels of the electrodes and were asymmetrically positioned within the tunneling junction and coupled with the $\left(\mathrm{Ga}_{2} \mathrm{O}_{3}\right)$ EGaIn electrode.

2) The difference of two orders of magnitude in the values of $R$ measured for the Fc and $\mathrm{BFc}$ functionalized dendrimers were most likely caused by the fact that the G1-PPI-(Fc) formed a discontinuous monolayer on the $\beta$ CD SAM causing 'thin area defects' (as only two of its four terminal moieties were able to bind to the $\beta \mathrm{CD}$ SAM, giving a surface coverage of approx. $90 \%$ per unit area due to the kinetics of the multivalent interactions, leaving approx. $10 \%$ of the $\beta \mathrm{CD}$ SAM free), whereas the $\mathrm{G} 1-\mathrm{PPI}-(\mathrm{BFc})_{4}$ formed a continuous monolayer (as three of its four terminal moieties were able to bind to the $\beta C D$ SAM, giving an approx. $100 \%$ coverage $).{ }^{[10]}$ As tunneling current $J\left(\mathrm{~A} / \mathrm{cm}^{2}\right)$ decays exponentially with the distance between the two electrodes $d(\AA)$, as approximated by the simple form of the Simmons equation, $J=J_{\mathrm{o}} \mathrm{e}^{-\beta \mathrm{d}}$ (where $J_{0}\left(\mathrm{~A} / \mathrm{cm}^{2}\right)$ is the current density flowing through the electrode-SAM interfaces in the hypothetical case of $d=0 \AA$, and $\beta\left(\AA^{-1}\right)$ is the decay constant), the "thin area defects', ${ }^{[11]}$ i.e. $\beta \mathrm{CD}$ SAM, dominated the measured tunneling current in the G1-PPI-(Fc) $)_{4}$ tunneling junction, and as the $\beta C D$ SAM does not rectify current, it decreased $R$. 
This Chapter describes experiments to test these hypotheses by using the well-defined $\beta C D$ SAM as a supramolecular platform to adsorb four different functionalized poly(amido amine) (PAMAM) dendrimers.

\subsection{Construction of Supramolecular Tunneling Junctions: Generation Zero Poly(amido amine) Dendrimers}

All junctions are described using nomenclature, $\mathrm{Au}^{\mathrm{TS}}-\beta C D S A M / \mathrm{X} /\left(\mathrm{Ga}_{2} \mathrm{O}_{3}\right) \mathrm{EGaIn}$ where "-" in $\mathrm{Au}^{\mathrm{TS}}-\beta \mathrm{CD}$ represents the non-covalent interface of the Au surface and the sulfur of the heptathioether functionalized $\beta C D$, “" represents the supramolecular host-guest interaction between the $\beta C D$ and the terminal functional group of the dendrimer, " $\mathrm{X}$ " represents the dendrimer, " $\left(\mathrm{Ga}_{2} \mathrm{O}_{3}\right)$ " represents the oxides of gallium present on the skin of the EGaIn and "//"represents the Van der Waals interactions at the interface between the terminal group of the molecular structure and the $\mathrm{Ga}_{2} \mathrm{O}_{3}$ on the skin of the EGaIn.

Figure 5.1 on page 137, shows the fabrication of the supramolecular tunneling junctions. A well-defined, quasi-hexagonally packed SAM of heptathioether-functionalized $\beta$ cyclodextrin $(\beta C D)^{[12]}$ is formed on $\mathrm{Au}^{\mathrm{TS}}$, thus creating the supramolecular platform. $\mathrm{Au}^{\mathrm{TS}}$ are used as the root mean square (rms) roughness of these surfaces is five times less than that of evaporated surfaces, ${ }^{[13]}$ thus ensuring the optimization of working devices. The use of dendrimers as guest molecules allows for multivalent host-guest interactions with the supramolecular platform to increase the stability of the supramolecular structures (single $\beta C D-F c$ interactions are too weak to obtain a fully covered $\beta C D$ SAM with native $F c$ ). The four G0-PAMAM dendrimers adsorbed to the supramolecular platform are G0-PAMAM- $(\mathrm{Fc})_{4}, \quad$ G0-PAMAM- $(\mathrm{Ad})_{4}$ (the corresponding control molecule), G0-PAMAM-((EG) $\left.)_{3}-\mathrm{Fc}\right)_{4}$ (with $(\mathrm{EG})_{3}$ being long tethers of three ethylene glycol units (EG) between the PAMAM core and the Fc functionalities), and its corresponding control molecule G0-PAMAM- $\left((\mathrm{EG})_{3}-\mathrm{Ad}\right)_{4}$. Contacting these with the $\left(\mathrm{Ga}_{2} \mathrm{O}_{3}\right)$ EGaIn top electrode gives the tunneling junctions $\mathrm{Au}^{\mathrm{TS}}-\beta C D S A M / G 0-P A M A M-(\mathrm{Fc})_{4} / /\left(\mathrm{Ga}_{2} \mathrm{O}_{3}\right) \mathrm{EGaIn}$ (5.1), $\mathrm{Au}^{\mathrm{TS}}-\beta C D S A M / G 0-P A M A M-(\mathrm{Ad})_{4} / /\left(\mathrm{Ga}_{2} \mathrm{O}_{3}\right)$ EGaIn (5.2), $\mathrm{Au}^{\mathrm{TS}}-\beta C D S A M / G 0-P A M A M-$ $\left((\mathrm{EG})_{3}-\mathrm{Fc}\right)_{4} / /\left(\mathrm{Ga}_{2} \mathrm{O}_{3}\right) \mathrm{EGaIn} \quad(5.3), \quad$ and $\quad \mathrm{Au}^{\mathrm{TS}}-\beta C D S A M / G 0-P A M A M-\left((\mathrm{EG})_{3}-\right.$ $\mathrm{Ad})_{4} / /\left(\mathrm{Ga}_{2} \mathrm{O}_{3}\right)$ EGaIn (5.4). 


\subsubsection{Testing of Hypothesis One}

Hypothesis 1: rectification occurs due to the Fc functional groups providing a low lying HOMO level with energies close to that of the Fermi levels of the electrodes, whilst being asymmetrically placed within the tunnelling junction and close to and coupled with the top $\left(\mathrm{Ga}_{2} \mathrm{O}_{3}\right)$ EGaIn electrode. As the supramolecular platform ( $\beta C D$ SAM) ensures that the dendrimer itself is always positioned asymmetrically within the EGaIn tunneling junction, this hypothesis was tested by changing the position of the Fc moieties of the dendrimer within the tunneling junction and determining the influence that the relative position has on the value of $R$ (where $R=|J(-2.0 \mathrm{~V})| /|J(+2.0 \mathrm{~V})|)$ obtained. This was carried out by investigating the $J(V)$ characteristics of, and hence value of $R$ obtained for the dendrimers

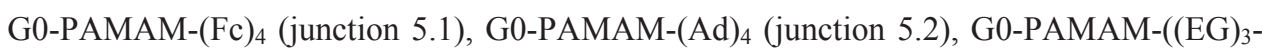
$\mathrm{Fc})_{4}$ (junction 5.3), and G0-PAMAM-((EG) $\left.3-\mathrm{Ad}\right)_{4}$ (junction 5.4), and comparing it to the value of $R$ obtained for the dendrimer G1-PPI-(Fc) $)_{4}$ investigated in Chapter 4 (junction 4.1). For G0-PAMAM- $\left((\mathrm{EG})_{3}-\mathrm{Fc}\right)_{4}$ (junction 5.3), the long tethers of three ethylene glycol units (EG) between the PAMAM core and the Fc terminal moieties allows for the complexation of all four Fc moieties with the supramolecular platform. ${ }^{[10 a, 10 b]}$ As all of the Fc moieties are bound to the supramolecular platform, there are no Fc moieties placed, close to and coupled with the $\left(\mathrm{Ga}_{2} \mathrm{O}_{3}\right)$ EGaIn top electrode. Instead, the $\left(\mathrm{Ga}_{2} \mathrm{O}_{3}\right)$ EGaIn top electrode will most likely contact the core of the PAMAM dendrimer (Figure 5.1, page 137 and 5.2, page 138). With the PAMAM core acting as an additional tunnel barrier between the layer of Fc moieties and the $\left(\mathrm{Ga}_{2} \mathrm{O}_{3}\right)$ EGaIn top electrode, these tunneling junctions are not expected to rectify currents. G0-PAMAM-((EG) $\left.)_{3}-\mathrm{Ad}\right)_{4}$ (junction 5.4) acts as a control for G0-PAMAM- $\left((\mathrm{EG})_{3}-\mathrm{Fc}\right)_{4}$ (junction 5.3), as replacing the Fc moieties with Ad moieties ensures that any possible characteristic trends seen in the $J(V)$ measurements of junction 5.3 are attributed to the Fc moieties and do not arise from the additional structural asymmetry created within the supramolecular tunneling junction by adsorbing a dendrimer to the platform. In contrast G0-PAMAM-(Fc) $)_{4}$ (junction 5.1) can only have three of its four Fc terminal moieties bound to the supramolecular platform, allowing the one free Fc moiety to be placed close to and coupled with the $\left(\mathrm{Ga}_{2} \mathrm{O}_{3}\right)$ EGaIn top electrode. ${ }^{[10 a, 10 b]}$ This molecular orientation within the tunneling junction is similar to that of G1-PPI-(Fc) $)_{4}$ investigated in Chapter 4 (junction 4.1) where two Fc moieties are complexed to the supramolecular platform and two are placed close to and coupled with the $\left(\mathrm{Ga}_{2} \mathrm{O}_{3}\right)$ EGaIn top electrode. As 
G1-PPI-(Fc) 4 (junction 4.1) was found to rectify currents, G0-PAMAM-(Fc) 4 (junction 5.1) is expected to rectify currents also. G0-PAMAM-(Ad) $)_{4}$ (junction 5.2) is used to act as a control, with the value of $R$ obtained for this junction not only being compared to $R$ found for G0-PAMAM-(Fc) $)_{4}$ (junction 5.1) but also to $R$ found for G1-PPI-(Ad) $)_{4}$ in Chapter 4 (junction 4.3) to ensure that any possible characteristic trends seen in the $J(V)$ measurements of junction 5.1 are attributed to the Fc moieties, and do not arise from the additional structural asymmetry created within the supramolecular tunneling junction by adsorbing a dendrimer to the platform.

Figure 5.1 (page 137): Idealized schematic of the fabrication process of the molecular junctions. The eutectic gallium-indium (EGaIn) top contact is represented exhibiting its inherent gallium oxide layer. Molecular structure of the core of the poly(amido amine) PAMAM dendrimers, with the red sphere representing the functional groups at the periphery of the 'standard' PAMAM dendrimers, and the jagged black line + red sphere representing the ethylene glycol linker + functional groups at the periphery of the ethylene glycol PAMAM dendrimers. The functionality of the dendrimer corresponds to junctions $\mathrm{Au}^{\mathrm{TS}}-\beta \mathrm{CDSAM} / \mathrm{G} 0$ PAMAM-(Fc) $)_{4} / /\left(\mathrm{Ga}_{2} \mathrm{O}_{3}\right)$ EGaIn $\quad(5.1), \quad \mathrm{Au}^{\mathrm{TS}}-\beta \mathrm{CDSAM} / \mathrm{G} 0-\mathrm{PAMAM}-(\mathrm{Ad})_{4} / /\left(\mathrm{Ga}_{2} \mathrm{O}_{3}\right)$ EGaIn $\quad(5.2), \quad \mathrm{Au}^{\mathrm{TS}}$ $\beta C D S A M / G 0-P A M A M-\left((E G)_{3}-F c\right)_{4} / /\left(G_{2} \mathrm{O}_{3}\right)$ EGaIn (5.3) and $\mathrm{Au}^{\mathrm{TS}}-\beta C D S A M / G 0-P A M A M-\left((E G)_{3^{-}}\right.$ $\mathrm{Ad})_{4} / /\left(\mathrm{Ga}_{2} \mathrm{O}_{3}\right)$ EGaIn (5.4), respectively. The $J(\mathrm{~V})$ measurements were performed by biasing the $\left(\mathrm{Ga}_{2} \mathrm{O}_{3}\right) \mathrm{EGaIn}$ top-electrode and connecting the Au bottom-electrode to ground. 


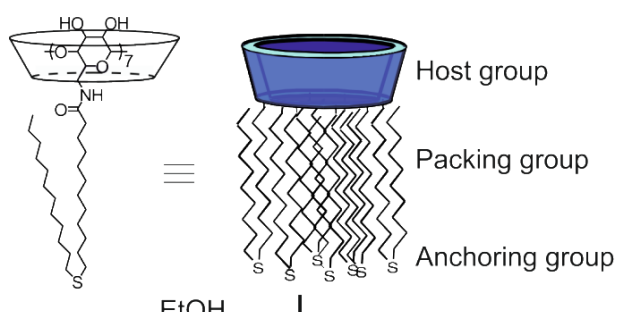

$\mathrm{EtOH}$

$16 \mathrm{hrs} / 60^{\circ} \mathrm{C}$

self assembly
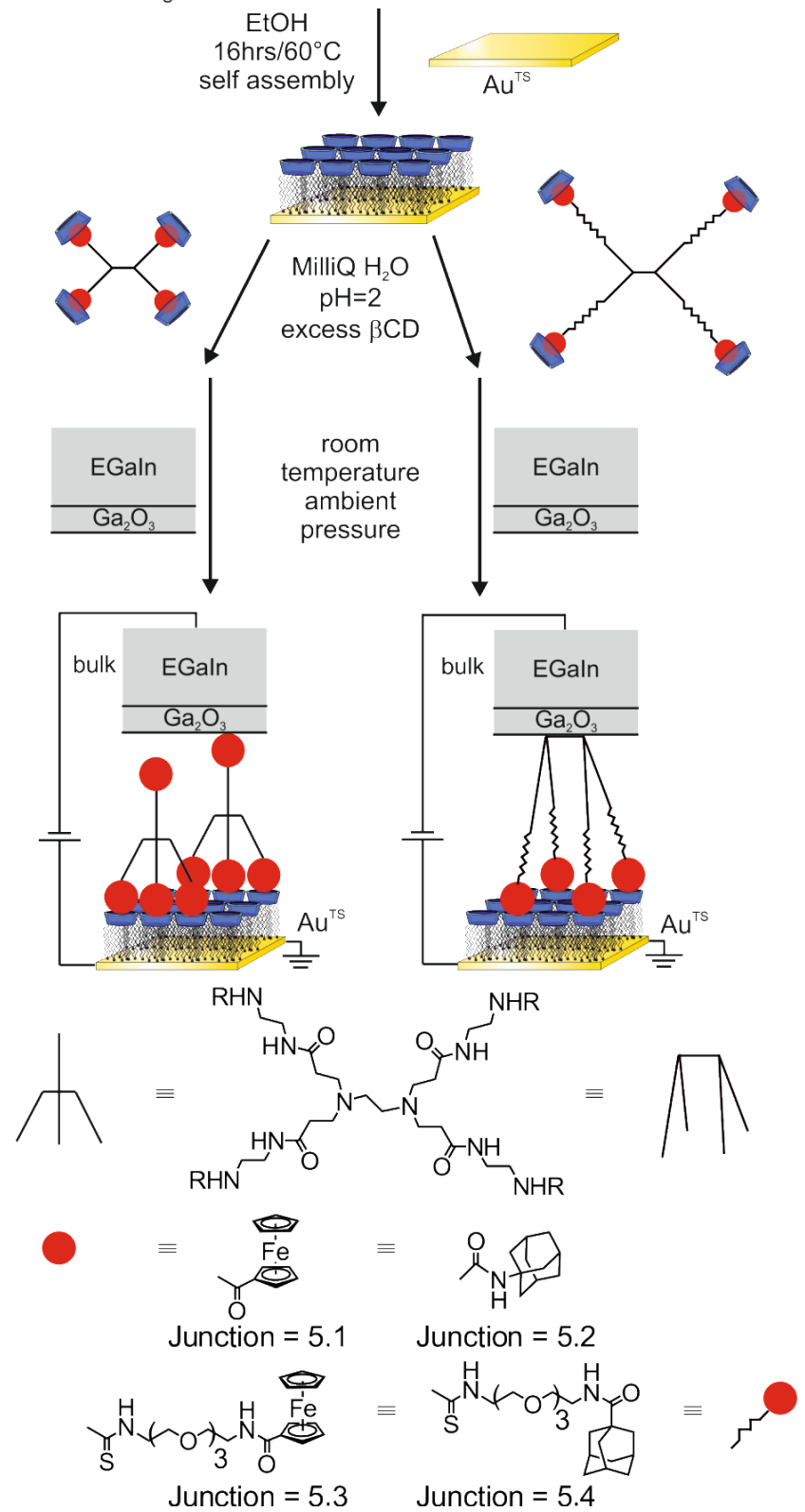
Negative Bias

$$
V=-2.0 \mathrm{~V}
$$
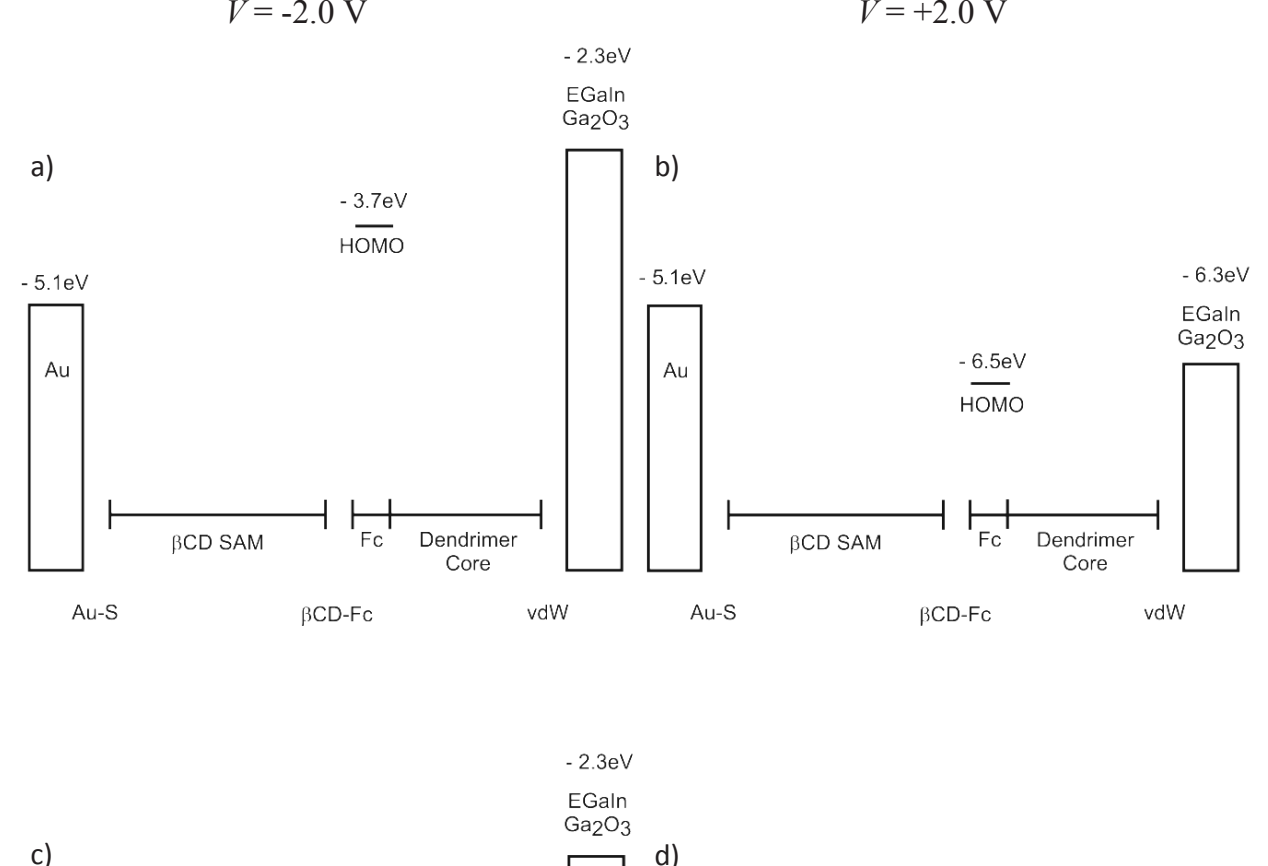

c)

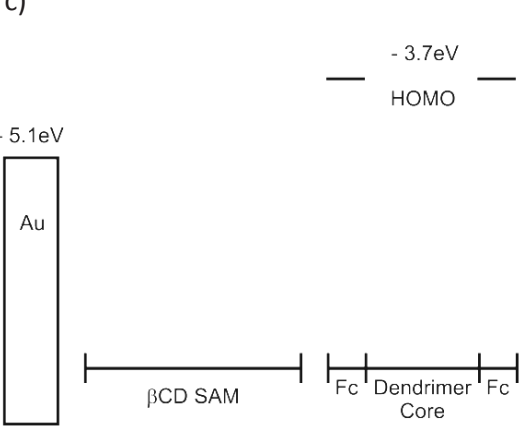

Au-S
$\mathrm{Ga}_{2} \mathrm{O}_{3}$

d)

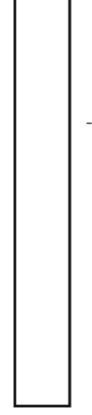

vdW
Positive Bias

$V=+2.0 \mathrm{~V}$
Au-S

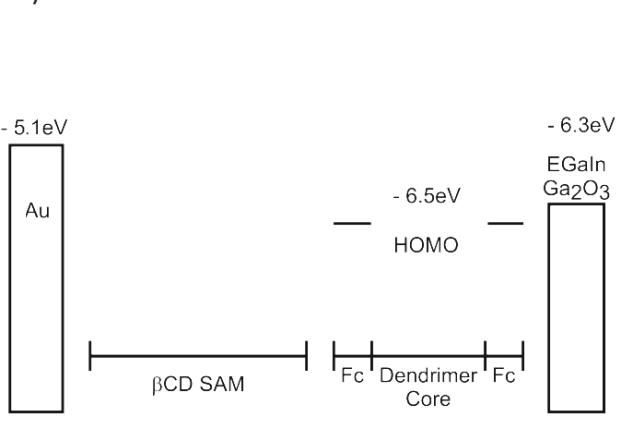

$\beta C D-F C$

vdW

Figure 5.2: The proposed molecular energy diagram for the supramolecular tunneling junctions; $\mathrm{Au}^{\mathrm{TS}}$ $\beta \mathrm{CDSAM} / \mathrm{G} 0-\mathrm{PAMAM}-\left((\mathrm{EG})_{3}-\mathrm{Fc}\right)_{4} / /\left(\mathrm{Ga}_{2} \mathrm{O}_{3}\right)$ EGaIn (junction 5.3) at $-2.0 \mathrm{~V}$ (a) and $+2.0 \mathrm{~V}$ (b), $\mathrm{Au}^{\mathrm{TS}}$ $\beta C D S A M / G 0-P A M A M-(F c)_{4} / /\left(G_{2} \mathrm{O}_{3}\right)$ EGaIn, (junction 5.1) at $-2.0 \mathrm{~V}$ (c) and $+2.0 \mathrm{~V}(\mathrm{~d}$ ). The positions of the HOMO levels in the diagram represent the position of the $\mathrm{Fc}$ terminal moiety within the tunneling junction. ${ }^{[14]}$ $\beta C D-F c=$ the supramolecular host-guest interaction between the $\beta C D$ SAM and the Fc terminal group of the dendrimer, $\mathrm{vdW}=$ van der Waals interface. 


\subsubsection{Testing of Hypothesis Two}

Hypothesis 2: the reason for the difference of two orders of magnitude in the values of $R$ measured for the Fc and BFc functionalized dendrimers in Chapter 4 was caused by the fact that the G1-PPI-(Fc) formed a discontinuous monolayer on the supramolecular platform causing 'thin area defects', whereas G1-PPI-(BFc) $)_{4}$ formed a continuous monolayer, thus minimizing the formation of thin area defects. This hypothesis is tested by comparing the value of $R$ obtained for G0-PAMAM-(Fc) $)_{4}$ (junction 5.1) with $R$ found for G1-PPI-(Fc) (junction 4.1) in Chapter 4. As mentioned above, G0-PAMAM-(Fc) 4 (junction 5.1) is able to complex three of the four terminal Fc moieties with the supramolecular platform. This results in a nearly $100 \%$ surface coverage and thus minimizes the formation of 'thin area defects'. Conversely, G1-PPI-(Fc) (junction 4.1) can only complex two of the four Fc moieties with the supramolecular platform due to its smaller PPI dendritic core, resulting in a surface coverage of approx. 90\% and subsequently suffers from 'thin area 'defects (Figure 5.3). The difference in dendrimer surface coverage is due to the absorbance kinetics of the dendrimers from a monovalent Fc- $\beta C D$ interaction in solution to a multivalent Fc- $\beta C D$ interaction on the supramolecular platform (see experimental). For G1-PPI-(Fc) 4 , the divalent Fc- $\beta C D$ interaction on the supramolecular platform is only slightly more kinetically stable than the monovalent interaction in solution, therefore limiting the coverage on the surface, whereas the trivalent interaction of G0-PMAM- $(\mathrm{Fc})_{4}$ on the supramolecular platform is more kinetically stable than the monovalent interaction, allowing for a full surface coverage. ${ }^{[10 \mathrm{a}, 10 \mathrm{~b}]}$ The remaining uncomplexed Fc moieties of the dendrimers (one for G0-PAMAM- $(\mathrm{Fc})_{4}$ and two for G1-PPI-( $\left.(\mathrm{Fc})_{4}\right)$ are placed close to and coupled with the $\left(\mathrm{Ga}_{2} \mathrm{O}_{3}\right)$ EGaIn top electrode (Figures 5.1 and 4.1), fulfilling the requirements for them to act as a molecular rectifier. However, as 'thin area defects' dominate the $J(V)$ characteristics, the value of $R$ obtained for G0-PAMAM- $(\mathrm{Fc})_{4}$ (junction 5.1) should be larger than that found for G1-PPI-(Fc) 4 (junction 4.1), as in this study a thin area defect is where the $\left(\mathrm{Ga}_{2} \mathrm{O}_{3}\right)$ EGaIn top electrode contacts the supramolecular platform below the dendrimer layer, with the supramolecular platform being found not to rectify currents in Chapter 4 . 


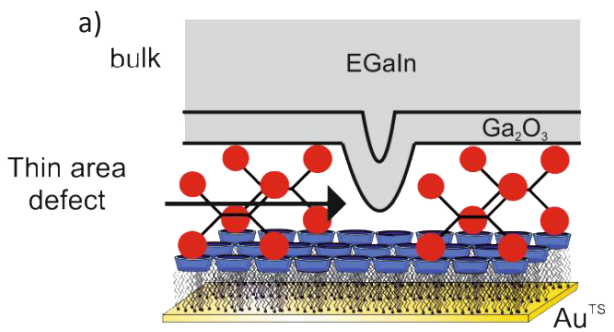

b)

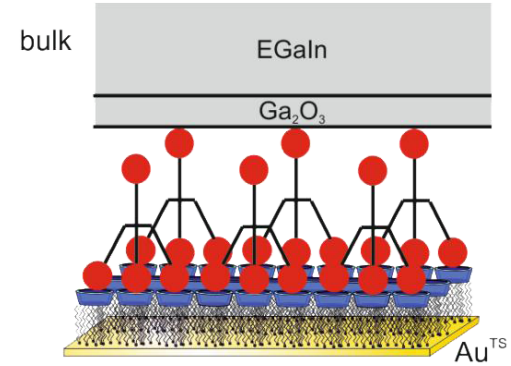

Figure 5.3: Schematic of the supramolecular tunneling junctions; (a) $\mathrm{Au}^{\mathrm{TS}}-\beta \mathrm{CDSAM} / \mathrm{G} 1-\mathrm{PPI}$ $(\mathrm{Fc})_{4} / /\left(\mathrm{Ga}_{2} \mathrm{O}_{3}\right)$ EGaIn (junction 4.1) with G1-PPI-( $\left.\mathrm{Fc}\right)_{4}$ forming an incomplete coverage on the supramolecular platform $\left(\beta C D\right.$ SAM) leading to thin area defects where the $\left(\mathrm{Ga}_{2} \mathrm{O}_{3}\right)$ EGaIn top electrode contacts the supramolecular platform, (b) $\mathrm{Au}^{\mathrm{TS}}-\beta \mathrm{CDSAM} / \mathrm{G} 0-\mathrm{PAMAM}-(\mathrm{Fc})_{4} / /\left(\mathrm{Ga}_{2} \mathrm{O}_{3}\right) \mathrm{EGaIn}$ (junction 5.1) with G0PAMAM- $(\mathrm{Fc})_{4}$ forming a complete coverage on the supramolecular platform ( $\beta C D$ SAM) and thus minimizing the formation of thin area defects.

\subsection{Results and Discussion}

This section describes the $J(V)$ data accumulation for junctions 5.1, 5.2, 5.3 and 5.4 and the statistical analysis of that data, with the results obtained being discussed as to whether they prove or disprove the two hypotheses.

\subsection{1 $J(V)$ Data Accumulation and Statistical Analysis}

The $\left(\mathrm{Ga}_{2} \mathrm{O}_{3}\right)$ EGaIn electrodes are large on the molecular scale $\left(100\right.$ to $\left.300 \mu \mathrm{m}^{2}\right),{ }^{[2 \mathrm{e}]}$ therefore these so-called large-area tunneling junctions contain in the order of $10^{6}-10^{7}$ molecules within the junction. The junctions in this study, as do all large-area junctions, contain defects that arise, for example, from surface roughness of the electrodes (step edges, grains), defects in the SAMs and impurities. ${ }^{[11,15]}$ The amount and types of defects vary from junction to junction and substrate to substrate, so it is essential to collect a statistically large amount of data from different $\mathrm{Au}^{\mathrm{TS}}$ substrates in order to conclude anything of significance. ${ }^{[2 \mathrm{f}]}$ Therefore, in this study statistically relevant numbers of data were collected (Table 5.1) allowing for a statistical analysis similar to that reported by Whitesides and co-workers ${ }^{[3 \mathrm{~b}, 3 \mathrm{~d}]}$ to be performed. (as explained in Chapter 3 and shown below, Figure 5.4 and 5.5). 
$J(\mathrm{~V})$ measurements were performed by biasing the $\left(\mathrm{Ga}_{2} \mathrm{O}_{3}\right)$ EGaIn top-electrode and connecting the Au bottom-electrode to ground. As explained in Chapters 3 and 4, an in depth statistical analysis was carried out for all supramolecular junction measurements. ${ }^{[3 \mathrm{~d}, 9]}$ Each average value of $|J|$ was calculated by plotting all the values of $|J|$ measured at each voltage value (step size $0.10 \mathrm{~V}$, in both sweep directions, with total sweep being $\pm 2.0 \mathrm{~V}$ ) into histograms, giving in total 82 individual histograms. All histograms were plotted on a $\log$ scale, giving a normal distribution, allowing the data to be fit with Gaussian curves. This gave the average $|J|$ (the log mean) and the error (one-log standard deviation, $68 \%$ of the data is within one log standard deviation of the log-mean) for each voltage value, allowing the construction of the average $|J|$ vs. $V$ scan. Figure 4.2 in Chapter 4 gives an example of how the averaged data points were calculated to obtain the average $|J|(V)$ scan with the error bars on the graph representing one log standard deviation from the log mean value, indicating between which $J$ values $68 \%$ of our total obtained $J$ measurements lie. Figures $5.4 \mathrm{a}, \mathrm{c}$ and $5.5 \mathrm{a}, \mathrm{c}$ show the semi log plot of the averaged (log mean) $|J|$ vs. $V$, for each EGaIn supramolecular junction structures investigated.

Each individual $\left(\mathrm{Ga}_{2} \mathrm{O}_{3}\right)$ EGaIn junction was also characterized as either a 'working junction' (junction that gave 20 reproducible scans $\pm 2.0 \mathrm{~V}$ with the current measured being within $3 \sigma_{\log }$ of the $\mu_{\log }$ value), a 'short' (a junction that produced an immediate ohmic response, or a junction that suddenly produced an ohmic response during the 20 scans \pm 2.0 $\mathrm{V}$ ), or an 'unstable junction' (during the 20 scans $\pm 2.0 \mathrm{~V}$ the current measured varied greater than $3 \sigma_{\log }$ from the $\mu_{\log }$ value; Table 5.1). Considering all junction structures, working junctions were found with an average yield of $80-85 \%$ (Table 1), which is in accordance with other molecular structures measured in $\left(\mathrm{Ga}_{2} \mathrm{O}_{3}\right)$ EGaIn junctions. ${ }^{[2 \mathrm{e}, 3 \mathrm{~d}, 9]}$ 
Table 5.1: Statistical overview of all $\left(\mathrm{Ga}_{2} \mathrm{O}_{3}\right)$ EGaIn junctions measured that contain generation zero PAMAM dendrimers (also includes the data of two generation one PPI dendrimers discussed in Chapter 4).

\begin{tabular}{|c|c|c|c|c|c|c|c|c|c|c|}
\hline $\begin{array}{l}\text { Molecular } \\
\text { Junction }^{\left[{ }^{[a]}\right.}\end{array}$ & $\begin{array}{l}\text { No. of } \\
\text { Substrates }{ }^{[b]}\end{array}$ & $\begin{array}{l}\text { No. of } \\
\text { Junctions } \\
\text { Created }^{(\mathrm{c})}\end{array}$ & $\begin{array}{l}\text { No. of } \\
\text { working } \\
\text { Junctions } \\
(\%)^{[d]}\end{array}$ & $\begin{array}{l}\text { No. of } \\
\text { Shorts } \\
{[\text { [c] }}\end{array}$ & $\begin{array}{l}\text { No. of } \\
\text { Unstable } \\
\text { Junctions } \\
{[\text { [r] }}\end{array}$ & $\begin{array}{l}\text { Total } \\
\text { Scans } \\
\text { [g] }\end{array}$ & $\mathrm{R}^{[\mathrm{h}]}$ & $\begin{array}{l}\text { Erтor } \\
(68 \%)^{[i]}\end{array}$ & $\begin{array}{l}\text { No. of } \\
\text { interactions } \\
\text { with } \beta C D \\
\text { SAM } \\
\text { [i] }\end{array}$ & $\begin{array}{l}\text { Surface } \\
\text { Coverage of } \\
\beta C D \text { SAM } \\
(\%)^{[k]}\end{array}$ \\
\hline 5.1 "Fc" & 5 & 22 & $20(91)$ & 1 & 1 & 400 & 12 & $2.2-66$ & 3 & $\sim 100$ \\
\hline 5.2 "Ad" & 3 & 17 & $14(82)$ & 2 & 1 & 280 & 0.83 & $0.17-4.1$ & 3 & $\sim 100$ \\
\hline 5.3 "(Fg) $)_{3}-\mathrm{Fc}^{\prime \prime}$ & 7 & 21 & $16(76)$ & 4 & 1 & 320 & 3.1 & $1.3-7.1$ & 4 & $\sim 100$ \\
\hline 5.4 "(Eg $)_{3}-\mathrm{Ad} "$ & 7 & 21 & $18(86)$ & 3 & 0 & 360 & 3.3 & $1.0-11$ & 4 & $\sim 100$ \\
\hline 4.1 "PPI-Fe" & 4 & 22 & $17(77)$ & 3 & 2 & 340 & 7.7 & $2.5-24$ & 2 & $\sim 89$ \\
\hline 4.3 "PPI-Ad" & 7 & 30 & $24(80)$ & 4 & 2 & 480 & 0.70 & $0.28-1.8$ & 2 & $>95$ \\
\hline
\end{tabular}

[a] $5.1=\mathrm{Au}^{\mathrm{TS}}-\beta \mathrm{CDSAM} / \mathrm{G} 0-\mathrm{PAMAM}-(\mathrm{Fc})_{4} /\left(\mathrm{Ga}_{2} \mathrm{O}_{3}\right)$ EGaIn, $\quad 5.2=\mathrm{Au}^{\mathrm{TS}}-\beta C D S A M / G 0-P A M A M-$ $(\mathrm{Ad})_{4} / /\left(\mathrm{Ga}_{2} \mathrm{O}_{3}\right)$ EGaIn, $\quad 5.3=\mathrm{Au}^{\mathrm{TS}}-\beta \mathrm{CDSAM} / \mathrm{G} 0-\mathrm{PAMAM}-\left((\mathrm{EG})_{3}-\mathrm{Fc}\right)_{4} / /\left(\mathrm{Ga}_{2} \mathrm{O}_{3}\right) \mathrm{EGaIn}, \quad 5.4=\mathrm{Au}^{\mathrm{TS}}-$ $\beta C D S A M / G 0-P A M A M-\left((E G)_{3}-A d\right)_{4} / /\left(G_{2} \mathrm{O}_{3}\right)$ EGaIn, $4.1=$ Au $^{\mathrm{TS}}-\beta \mathrm{CDSAM} / \mathrm{G} 1-\mathrm{PPI}-(\mathrm{Fc})_{4} / /\left(\mathrm{Ga}_{2} \mathrm{O}_{3}\right)$ EGaIn (from Chapter 4), $4.3=\mathrm{Au}^{\mathrm{TS}}-\beta \mathrm{CDSAM} / \mathrm{G} 1-\mathrm{PPI}-(\mathrm{Ad})_{4} /\left(\mathrm{Ga}_{2} \mathrm{O}_{3}\right) \mathrm{EGaIn}$ (from Chapter 4 ). $\quad[\mathrm{b}]=1 \mathrm{~cm} \times 1 \mathrm{~cm}$ $\mathrm{Au}^{\mathrm{TS}}$ surface on glass. [c] $=\left(\mathrm{Ga}_{2} \mathrm{O}_{3}\right)$ EGaIn top electrode contacting the molecular monolayer immobilized on the $\mathrm{Au}^{\mathrm{TS}}$ surface. $[\mathrm{d}]=$ junctions that gave 20 reproducible scans $\pm 2.0 \mathrm{~V}$ with the current measured being within $3 \sigma_{\log }$ of the $\mu_{\log }$ value, (number of working junctions/number of junctions created) [e] = a junction that produced an immediate ohmic response, or a junction that produced an ohmic response during the 20 scans \pm $2.0 \mathrm{~V}$. [f] = during the 20 scans $\pm 2.0 \mathrm{~V}$ the current measured varied greater than $3 \sigma_{\log }$ from the $\mu_{\log }$ value. [g] $=$ a voltage sweep, $0.0 \mathrm{~V} \rightarrow+2.0 \mathrm{~V} \rightarrow 0.0 \mathrm{~V} \rightarrow-2.0 \mathrm{~V} \rightarrow 0.0 \mathrm{~V}$. [h] $=|J(-2.0 \mathrm{~V})||J(+2.0 \mathrm{~V})|$. [i] $=$ one logstandard deviation, $68 \%$ of the data is within one $\log$ standard deviation of the log-mean. $[j]=$ amount of dendrimer terminal moieties that form host-guest interactions with the $\beta C D S A M$, out of a possible total of four. $[\mathrm{k}]=$ surface coverage $(\%)$ of the dendrimer adsorbed to the supramolecular platform. ${ }^{[10 \mathrm{a}, 10 \mathrm{~b}, 16]}$ 
Junction $5.1=\mathrm{Au}^{\mathrm{TS}}-\beta \mathrm{CDSAM} / \mathrm{G} 0-\mathrm{PAMAM}-(\mathrm{Fc})_{4} / /\left(\mathrm{Ga}_{2} \mathrm{O}_{3}\right) \mathrm{EGaIn}$

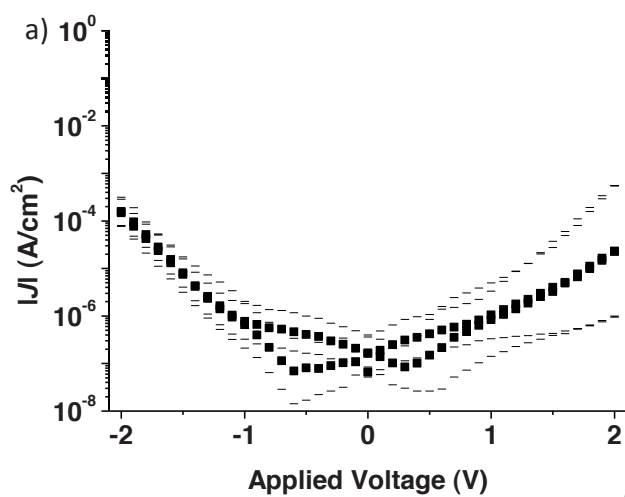

b) 35

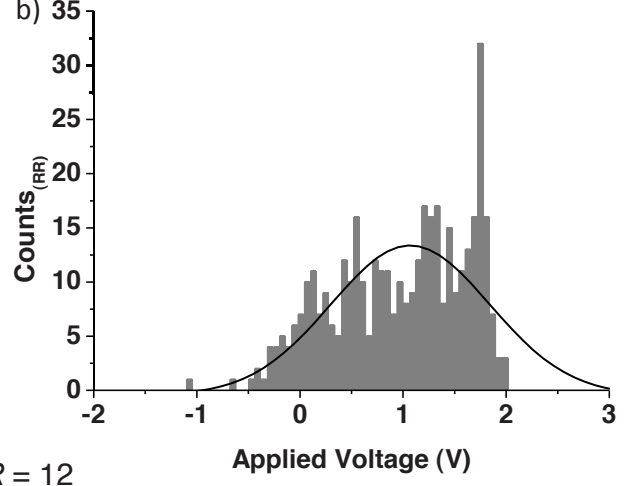

$R=12$

$\sigma_{\log }=5.5$

$2.2<R(68 \%)<66$

Junction 5.2 = $\mathrm{Au}^{\mathrm{TS}}-\beta C D S A M / G 0-P A M A M-(\mathrm{Ad})_{4} / /\left(\mathrm{Ga}_{2} \mathrm{O}_{3}\right)$ EGaIn

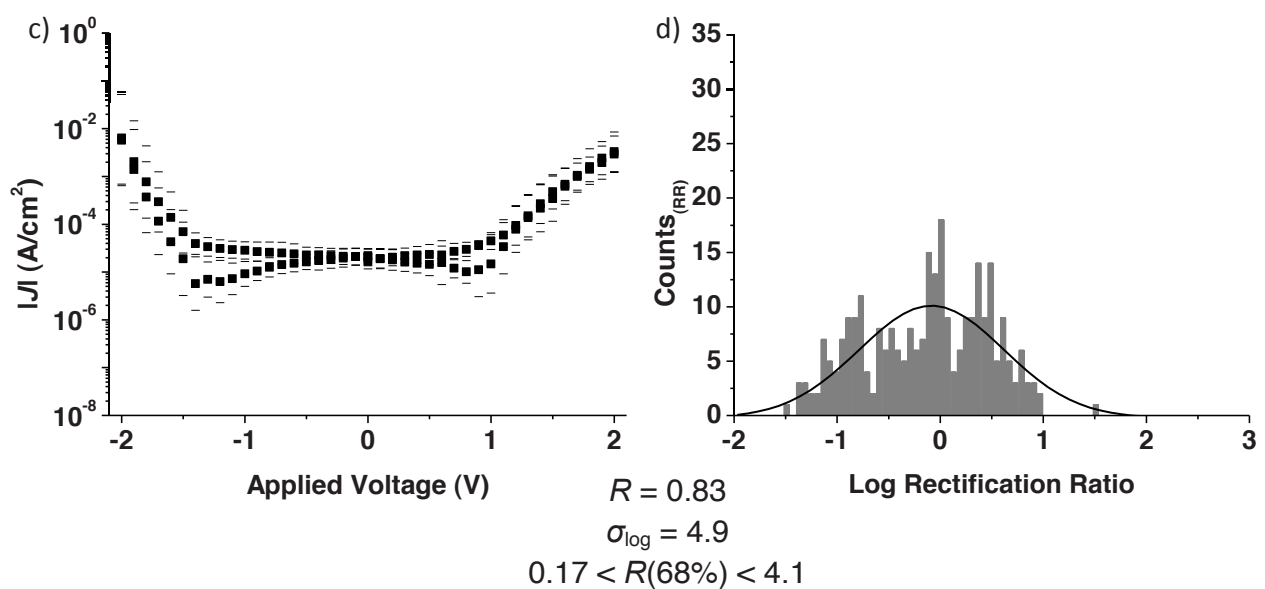

Figure 5.4: Data obtained for the supramolecular tunneling junctions containing dendrimers with three terminal functional moieties bound to the supramolecular platform, giving approx. $100 \%$ surface coverage, with the remaining free terminal moiety being asymmetrically positioned within the tunneling junction, coupled with the $\left(\mathrm{Ga}_{2} \mathrm{O}_{3}\right)$ EGaIn electrode. Semi-log plot of the averaged absolute current density vs. voltage $(|J|$ vs. $V)$ for; (a) $\mathrm{Au}^{\mathrm{TS}}-\beta C D S A M / G 0-P A M A M-(F c)_{4} /\left(\mathrm{Ga}_{2} \mathrm{O}_{3}\right) \mathrm{EGaIn}$ (junction 5.1) and (c) $\mathrm{Au}^{\mathrm{TS}}$ $\beta C D S A M / G 0-P A M A M-(A d)_{4} / /\left(\mathrm{Ga}_{2} \mathrm{O}_{3}\right)$ EGaIn (junction 5.2), with the error bars representing one log-standard deviation, $68 \%$ of the data is within one $\log$ standard deviation of the log-mean. Histograms of $\log$ Rectification ratio ( $R$ ) for; (b) $\mathrm{Au}^{\mathrm{TS}}-\beta \mathrm{CDSAM} / \mathrm{G} 0-\mathrm{PAMAM}-(\mathrm{Fc})_{4} /\left(\mathrm{Ga}_{2} \mathrm{O}_{3}\right) \mathrm{EGaIn}$ (junction 5.1) and (d) $\mathrm{Au}^{\mathrm{TS}}$ $\beta C D S A M / G 0-P A M A M-(A d)_{4} /\left(G_{2} \mathrm{O}_{3}\right)$ EGaIn (junction 5.2). All histograms were fitted with a Gaussian to obtain the log mean $\left(\mu_{\log }\right)$ and $\log$ standard deviation $\left(\sigma_{\log }\right)$ thus allowing for the calculation of $R(68 \%)$. 


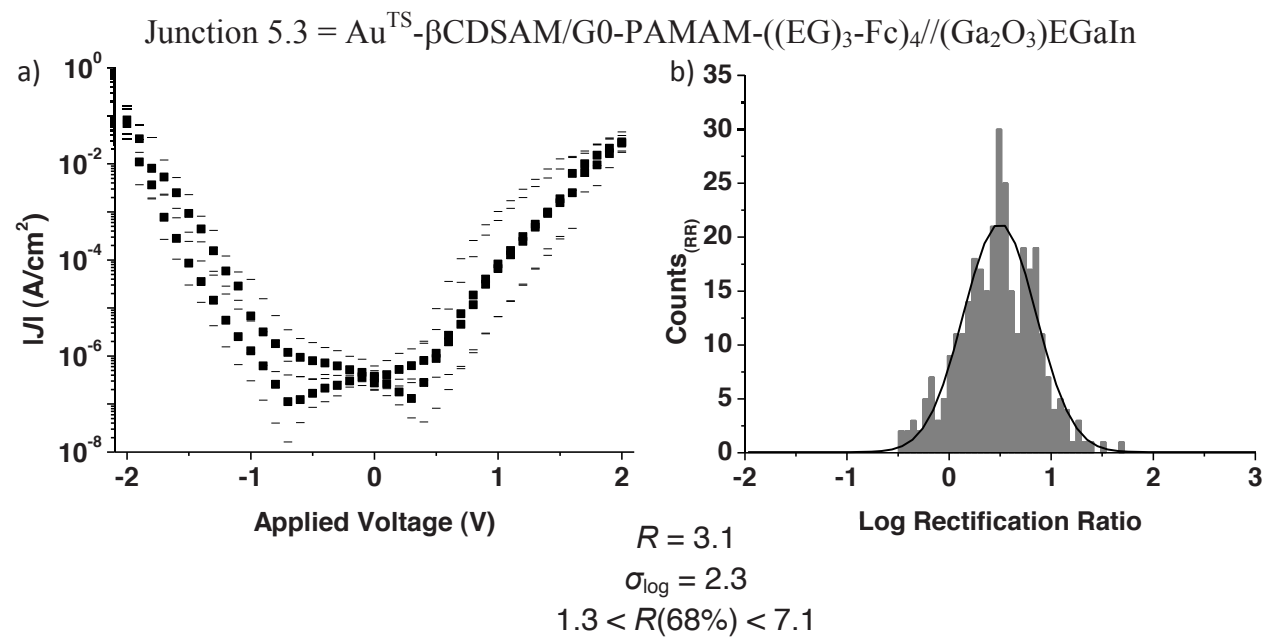

Junction 5.4 = $\mathrm{Au}^{\mathrm{TS}}-\beta \mathrm{CDSAM} / \mathrm{G} 0-$ PAMAM- $\left((\mathrm{EG})_{3}-\mathrm{Ad}\right)_{4} / /\left(\mathrm{Ga}_{2} \mathrm{O}_{3}\right)$ EGaIn
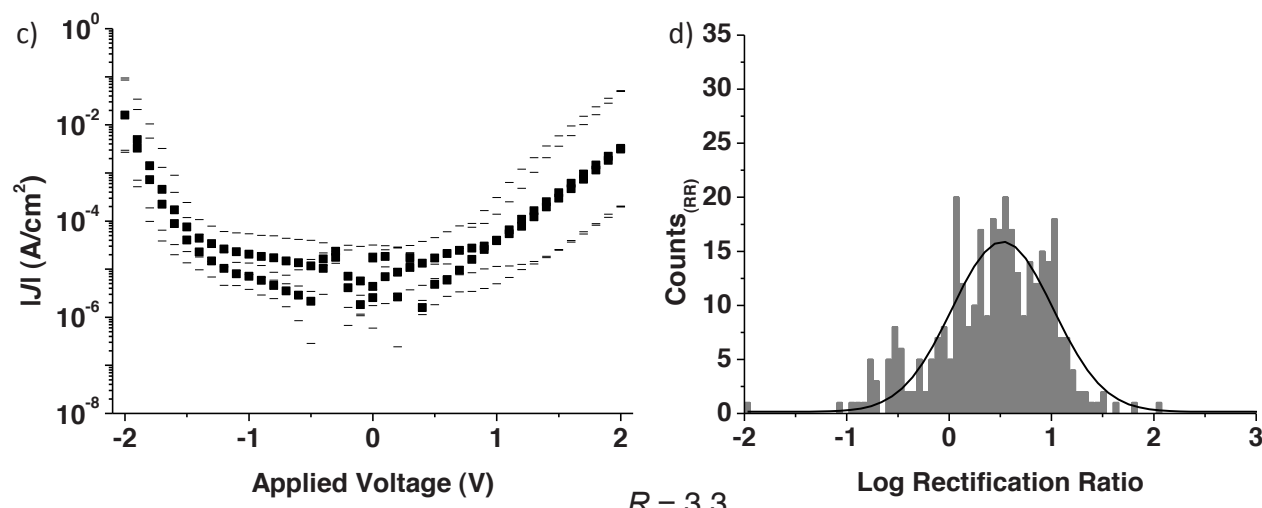

$$
\begin{gathered}
R=3.3 \\
\sigma_{\log }=3.2
\end{gathered}
$$$$
1.0<R(68 \%)<11
$$

Figure 5.5: Data obtained for the supramolecular tunneling junctions containing dendrimers with all four terminal functional moieties bound to the supramolecular platform. Semi-log plot of the averaged absolute current density vs. voltage ( $J \mid$ vs. $V$ ) for; (a) $\mathrm{Au}^{\mathrm{TS}}-\beta \mathrm{CDSAM} / \mathrm{G} 0-\mathrm{PAMAM}-\left((\mathrm{EG})_{3}-\mathrm{Fc}\right)_{4} /\left(\mathrm{Ga}_{2} \mathrm{O}_{3}\right) \mathrm{EGaIn}$ (junction 5.3) and (c) $\mathrm{Au}^{\mathrm{TS}}-\beta \mathrm{CDSAM} / \mathrm{G} 0-\mathrm{PAMAM}-\left((\mathrm{EG})_{3}-\mathrm{Ad}\right)_{4} /\left(\mathrm{Ga}_{2} \mathrm{O}_{3}\right) \mathrm{EGaIn}$ (junction 5.4), with the error bars representing one log-standard deviation, $68 \%$ of the data is within one log standard deviation of the logmean. Histograms of $\log$ Rectification ratio $(R)$ for; (b) $\mathrm{Au}^{\mathrm{TS}}-\beta \mathrm{CDSAM} / \mathrm{G} 0-\mathrm{PAMAM}-\left((\mathrm{EG})_{3^{-}}\right.$ $\mathrm{Fc})_{4} / /\left(\mathrm{Ga}_{2} \mathrm{O}_{3}\right)$ EGaIn (junction 5.3) and (d) $\mathrm{Au}^{\mathrm{TS}}-\beta \mathrm{CDSAM} / \mathrm{G} 0$-PAMAM-((EG) $\left.)_{3}-\mathrm{Ad}\right)_{4} / /\left(\mathrm{Ga}_{2} \mathrm{O}_{3}\right)$ EGaIn (junction 5.4). All histograms were fitted with a Gaussian to obtain the $\log$ mean $\left(\mu_{\log }\right)$ and $\log$ standard deviation $\left(\sigma_{\log }\right)$ thus allowing for the calculation of $R(68 \%)$. 
In summary, Figures 5.4a,c and 5.5a,c show the averaged $J(V)$ scans for all junctions investigated. The value of $R$ [Eq. (4.1)] was calculated for each individual scan for each junction, and statistically analyzed in the same manner as the averaged $J(V)$ curves (Figures 5.4b,d and 5.5b,d; also see Chapters 3 and 4). Junction 5.1 gave the largest $R$ value of 12 $\left(\sigma_{\log }=5.5\right)$, junctions 5.3 and 5.4 gave small values of $R$ similar to each other of $3.1\left(\sigma_{\log }=\right.$ 2.3 ) and $3.3\left(\sigma_{\log }=3.2\right)$, respectively, and junction 5.2 gave the $R$ value closest to unity of $0.83\left(\sigma_{\log }=4.9\right)$ (See Table 5.1) and additionally unlike the other three supramolecular junctions gave larger values of $J$ at a positive bias than at a negative bias. These results confirm hypothesis 1 , that rectification occurs due to the Fc functional groups providing a low lying HOMO level with energies close to that of the Fermi levels of the electrodes, whilst being asymmetrically placed within the tunneling junction and close to and coupled with the top $\left(\mathrm{Ga}_{2} \mathrm{O}_{3}\right)$ EGaIn electrode. Also, the results show some agreement with hypothesis 2 , that the reason for the difference of two orders of magnitude in the values of $R$ measured for the Fc and BFc functionalized dendrimers in Chapter 4 was caused by the fact that the G1-PPI-(Fc) formed a discontinuous monolayer on the supramolecular platform causing 'thin area defects', whereas G1-PPI-(BFc) $)_{4}$ formed a continuous monolayer, thus minimizing the formation of thin area defects.

\subsubsection{Verification of Hypothesis One}

For rectification to occur, the presence of $\mathrm{Fc}$ moieties are indeed important for providing a low lying HOMO level, that is a HOMO level with energies close to that of the Fermi levels of the electrodes. Additionally, this HOMO level must be placed asymmetrically within the tunneling junction and close to and coupled with one of the electrodes (in this case the $\left(\mathrm{Ga}_{2} \mathrm{O}_{3}\right)$ EGaIn top-electrode) (figure 5.2c and d). Junctions that were found to significantly rectify currents contain dendrimers G0-PAMAM- $(\mathrm{Fc})_{4}$ (junction 5.1) $\left(R=12\left(\sigma_{\log }=5.5\right)\right)$ and G1-PPI-(Fc) $)_{4}$ (junction 4.1) $\left(R=7.7\left(\sigma_{\log }=3.1\right)\right)$. The HOMO level for both of these dendrimers, estimated from Cyclic Voltammetry $(\mathrm{CV})$ is $-5.1 \mathrm{eV}^{[10 \mathrm{~b}]}$ which fulfills the energy level requirement, as the Fermi levels of $\mathrm{Au}$ and $\left(\mathrm{Ga}_{2} \mathrm{O}_{3}\right)$ EGaIn are approximately $-5.1 \mathrm{eV}$ and $-4.3 \mathrm{eV}$, respectively. Also, these dendrimers fulfill the second requirement as the supramolecular platform ensures that they are always positioned asymmetrically within the EGaIn tunneling junction; moreover, they also have a trivalent (G0-PAMAM-(Fc) $\left.)_{4}\right)$ or divalent (G1-PPI- $\left.(\mathrm{Fc})_{4}\right)$ interaction with the supramolecular platform allowing free Fc terminal moiety/ies to be positioned close to and coupled with the 
$\left(\mathrm{Ga}_{2} \mathrm{O}_{3}\right)$ EGaIn top-electrode (Figure 5.1 and 5.2c,d). Additionally, G0-PAMAM-(Ad) $)_{4}$ (junction 5.2) was found to not rectify currents $\left(R=0.83\left(\sigma_{\log }=4.9\right)\right)$, proving that the rectification seen in G0-PAMAM- $(\mathrm{Fc})_{4}$ (junction 5.1) is attributed to the Fc moieties. In contrast, the junction containing the G0-PAMAM- $\left((\mathrm{EG})_{3}-\mathrm{Fc}\right)_{4}$ dendrimer (junction 5.3) did not significantly rectify current $\left(\mathrm{R}=3.1\left(\sigma_{\mathrm{log}}=2.3\right)\right)$ as the additional ethylene glycol linker between the PAMAM core and the Fc terminal moiety enables the dendrimer to have a tetravalent interaction with the supramolecular platform. This means that in these junctions even though the dendrimer itself is positioned asymmetrically within the junction, all of the dendrimers terminal functional moieties are bound to the supramolecular platform, leaving no free Fc moieties to be positioned close to and coupled with the top electrode. Thus, the dendritic core with the ethylene glycol linker is in contact with the top-electrode rather than the Fc moieties (Figures 5.1 and 5.2a,b) which are embedded within the supramolecular platform. Or in other words, the Fc units are decoupled from the top-electrodes resulting in the small values of $R$. The theory that the $\left(\mathrm{Ga}_{2} \mathrm{O}_{3}\right)$ EGaIn top electrode is contacted to the core of the G0-PAMAM- $\left((\mathrm{EG})_{3}-\mathrm{Fc}_{4}\right.$ dendrimer in junction 5.3 is additionally supported by junction 5.4 (which contains the G0-PAMAM- $\left((E G)_{3}-A d\right)_{4}$ dendrimer) giving a similar value of $R\left(R=3.3\left(\sigma_{\log }=3.2\right)\right)$.

\subsubsection{Agreement with Hypothesis Two}

It is strongly suggested that the trivalent interaction of the G0-PAMAM- $(\mathrm{Fc})_{4}$ dendrimer with the supramolecular platform is why junction 5.1 rectifies $\left(R=12\left(\sigma_{\log }=5.5\right)\right)$ more than the junctions containing the G1-PPI-(Fc) 4 dendrimer (junction 4.1) $\left(R=7.7\left(\sigma_{\log }=3.1\right)\right)$ that have only a divalent interaction. The divalent interaction with the supramolecular platform only allows for a $90 \%$ coverage, leaving $10 \%$ of the supramolecular platform exposed, which in turn decreases the value of $R$ obtained as explained in Chapter 4, and above in section 5.2.2, and shown above in Figure 5.3. The trivalent interaction of the G0-PAMAM-(Fc) 4 dendrimer (junction 5.1) allows for a densely packed dendrimer layer to be formed on the supramolecular platform. Therefore, the "thin area" defects are minimized and charge transport across the dendrimer dominates the characteristics seen in the $J(V)$ measurements, and thus rectifies more. The reason why the junction containing the G0-PAMAM-(Fc) $)_{4}$ dendrimer (junction 5.1) rectifies less than junctions containing the G1PPI-(BFc) ${ }_{4}$ dendrimer (junction 4.2, in Chapter 4) that also have three CD-Fc interactions may originate from the different densities of $\mathrm{Fc}$ units present in both types of junctions. 146 


\subsubsection{Comparison of Control Dendrimers}

Interestingly, junction 5.2 containing the dendrimer G0-PAMAM-(Ad) 4 gave a similar value of $R\left(0.83\left(\sigma_{\log }=4.9\right)\right)$ to that found for junction 4.3 (in Chapter 4$)$ containing the dendrimer G1-PPI-(Ad) $4\left(\mathrm{R}=0.70\right.$ with $\left.\sigma_{\log }=2.5\right)$. Apparently, neither having a different dendrimer core, a different number of Ad- $\beta C D$ interactions per dendrimer (two for the PPI and three for the PAMAM dendrimer) with the supramolecular platform, or a different number of unbound Ad moieties (two for the PPI and one for the PAMAM dendrimer), affected the value of $R$ obtained. In contrast these differences in dendrimer structures for the

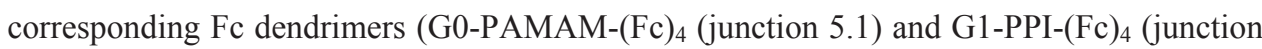
4.1) change the value or $R$ obtained $\left(R=12\left(\sigma_{\log }=5.5\right)\right.$ Vs. $\left(R=7.7\left(\sigma_{\log }=3.1\right)\right.$, respectively)). Therefore, the values of $R$ obtained for junction 5.1 and junction 4.1, are due to the presence of the Fc moieties.

\subsection{Conclusion}

In summary, the supramolecular $\beta C D$ platform provides the ability to adsorb various functionalized PAMAM dendrimers, to form well defined and stable $\left(\mathrm{Ga}_{2} \mathrm{O}_{3}\right)$ EGaIn supramolecular tunneling junctions, which allowed for the accumulation of statistically relevant numbers of data. By changing the core of the dendrimer from PPI (Chapter 4) to PAMAM and the distance between the terminal functional moieties, it was possible to change the relative position of the terminal functional moiety within the supramolecular tunneling junction and change the relative packing of the dendrimer on the supramolecular platform. The G0-PAMAM-(Fc) $)_{4}$ dendrimer which produced a densely packed layer on the supramolecular platform and is asymmetrically placed within the tunneling junction with one terminal functional group positioned close to and coupled with the $\left(\mathrm{Ga}_{2} \mathrm{O}_{3}\right) \mathrm{EGaIn}$ top-electrode, gave the largest value of $R$ in this study, with the corresponding $\mathrm{Ad}$ functionalized dendrimer, G0-PAMAM- $(\mathrm{Ad})_{4}$ not rectifying currents. Interestingly, both dendrimers G0-PAMAM- $\left((E G)_{3}-F c\right)_{4}$ and G0-PAMAM- $\left((E G)_{3}-A d\right)_{4}$, which also formed a densely packed layer on the supramolecular platform and were asymmetrically positioned within the tunneling junction but did not have a terminal functional moiety positioned close to and coupled with the $\left(\mathrm{Ga}_{2} \mathrm{O}_{3}\right)$ EGaIn top-electrode, gave similar small values of $R$, even though one dendrimer (G0-PAMAM-(EG) $\left.{ }_{3}-\mathrm{Fc}\right)_{4}$ ) contained a Fc terminal functional moiety which has a HOMO level close to that of the Fermi levels of the electrodes. These results 
allowed for the verification of hypothesis 1 , and showed a strong agreement with hypothesis 2. Additionally, the results obtained are in agreement with the theory of molecular rectification proposed by Williams and co-workers, ${ }^{[6-8]}$ Baranger and co-workers, and Ford and co-workers ${ }^{[8]}$ and experimentally shown by Whitesides and co-workers, ${ }^{[3 \mathrm{~b}-\mathrm{d}]}$ who state that for molecular rectification to occur there must be a low lying HOMO or LUMO level with energies close to that of the Fermi levels of the electrodes, and that this HOMO or LUMO level must be placed asymmetrically within the junction and coupled with one of the electrodes. 


\subsection{Experimental}

\section{Materials:}

\section{Synthesis of heptathioether-functionalized $\beta$-cyclodextrin}

Preparation of heptathioether-functionalized $\beta$-cyclodextrin ${ }^{[12 a]}$ was described previously and characterized with Nuclear Magnetic Resonance Spectroscopy ( ${ }^{1} \mathrm{H}$ NMR) and Mass Spectrometry (MALDI ToF and ESI-MS), with all compounds yielding similar results to what has been previously published. Eutectic Gallium Indium was used as purchased from Aldrich.

\section{Synthesis and Characterization of Dendrimers G0-PAMAM-((EG) $)_{3}$-Ad) ${ }_{4}$}
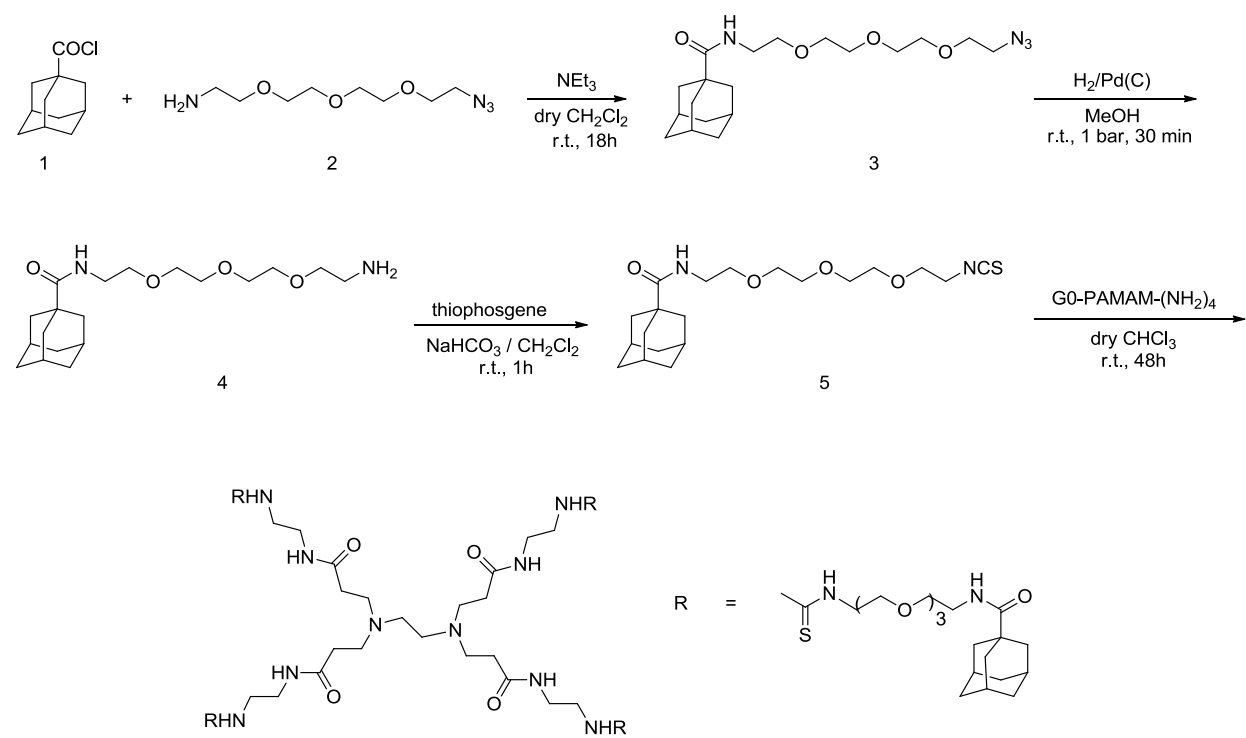

\section{Step 1: Synthesis of Ad-EG-N 3 (3)}

11-Azido-3,6,9-trioxaundecan-1-amine (2) (1 mmol, $218 \mathrm{mg}$ ) was dissolved in dry dichloromethane $(20 \mathrm{~mL})$ and $\mathrm{NEt}_{3}(2 \mathrm{mmol}, 202 \mathrm{mg}, 270 \mu \mathrm{L})$ was added. The solution was cooled to $0^{\circ} \mathrm{C}$ using an ice bath, and a solution of adamantane-carboxylic acid chloride (1) (1.5 mmol, $299 \mathrm{mg}$ in $20 \mathrm{~mL}$ of dry dichloromethane was added dropwise. The ice bath was removed and the reaction mixture was allowed to reach the room temperature and stirred overnight. After that time, the reaction mixture was washed with aqueous $0.1 \mathrm{M} \mathrm{HCl}$ solution 
( $3 \times 25 \mathrm{~mL})$, aqueous saturated $\mathrm{NaHCO}_{3}(3 \times 25 \mathrm{~mL})$, and water $(3 \times 25 \mathrm{~mL})$. The organic layer was dried over $\mathrm{Mg} \mathrm{SO}_{4}$ and the solvent was evaporated under reduced pressure. The crude reaction product was purified by flash column chromatography (silicagel, $\left.\mathrm{CH}_{2} \mathrm{Cl}_{2} / \mathrm{MeOH} 90 / 10\right)$ and isolated as a colorless oil. Yield: $78 \%(0.78 \mathrm{mmol}, 296 \mathrm{mg}) .{ }^{1} \mathrm{H}$ $\operatorname{NMR}\left(600 \mathrm{MHz}, \mathrm{CDCl}_{3}\right) \delta=6.12(\mathrm{bs}, 1 \mathrm{H}, \mathrm{CONH}), 3.68\left(\mathrm{~s}, 8 \mathrm{H}, \mathrm{OCH}_{2} \mathrm{CH}_{2} \mathrm{OCH}_{2} \mathrm{CH}_{2} \mathrm{O}\right)$, $3.64\left(\mathrm{~m}, 2 \mathrm{H}, \mathrm{OCH}_{2} \mathrm{CH}_{2} \mathrm{~N}_{3}\right), 3.55\left(\mathrm{t}, J=5.0 \mathrm{~Hz}, 2 \mathrm{H}, \mathrm{NHCH}_{2} \mathrm{CH}_{2} \mathrm{O}\right), 3.45(\mathrm{q}, J=5.0 \mathrm{~Hz}, 2 \mathrm{H}$, $\mathrm{NHCH}_{2} \mathrm{CH}_{2} \mathrm{O}$ ), 3.40 (t, $\left.J=5.0 \mathrm{~Hz}, 2 \mathrm{H}, \mathrm{OCH}_{2} \mathrm{CH}_{2} \mathrm{~N}_{3}\right), 2.04\left(\mathrm{~s}, 3 \mathrm{H}, \mathrm{CHCH}_{2}\right), 1.86(\mathrm{~s}, 6 \mathrm{H}$, $\left.\mathrm{CHCH}_{2} \mathrm{CCONH}\right), 1.72\left(\mathrm{~m}, J=15.5 \mathrm{~Hz}, 6 \mathrm{H}, \mathrm{CHCH}_{2} \mathrm{CH}\right) .{ }^{13} \mathrm{C} \mathrm{NMR}\left(75 \mathrm{MHz}, \mathrm{CDCl}_{3}\right) \delta=$ 178.3, 71.0, 70.8, 70.5, 70.3, 70.182, 50.9, 40.8, 39.4, 39.2, 36.8, 28.4. ESI-TOF Calculated for $\mathrm{C}_{19} \mathrm{H}_{32} \mathrm{~N}_{4} \mathrm{O}_{4}: m / z 380.1$ Found: $m / z 381.1[\mathrm{M}+\mathrm{H}]$.

\section{Step 2: Synthesis of Ad-EG-NH 2 (4)}

Ad-EG-N $\mathrm{N}_{3}$ (3) $(0.1 \mathrm{mmol}, 38 \mathrm{mg})$ was dissolved in $20 \mathrm{~mL}$ of methanol, and $\mathrm{Pd} / \mathrm{C}$ (0.01 eq, $0.01 \mathrm{mmol}, 10 \mathrm{mg}$ of $10 \% \mathrm{Pd} / \mathrm{C}$ ) was added. Nitrogen was bubbled through the solution for $5 \mathrm{~min}$, and then the reaction mixture was stirred at room temperature under $\mathrm{H}_{2}$ (normal pressure) for $30 \mathrm{~min}$ (monitored by TLC and IR spectroscopy- the disappearance of the characteristic azide peak at $2098 \mathrm{~cm}^{-1}$ - to assess the complete consumption of the starting material). The reaction mixture was filtered over a Celite pad, and the solvent was evaporated to afford the Ad-EG- $\mathrm{NH}_{2}$ as a colorless oil, which was used in the next step without further purification. Yield: $100 \%(0.1 \mathrm{mmol}, 35 \mathrm{mg}) .{ }^{1} \mathrm{H}-\mathrm{NMR}\left(300 \mathrm{MHz}, \mathrm{CDCl}_{3}\right)$ : $6.25(\mathrm{bd}, 1 \mathrm{H}, \mathrm{CONH}), 3.67-3.59\left(\mathrm{~m}, 9 \mathrm{H}, \mathrm{OCH}_{2} \mathrm{CH}_{2} \mathrm{OCH}_{2} \mathrm{CH}_{2} \mathrm{O}\right.$ and $\left.\mathrm{OCH}_{2} \mathrm{CH}_{2} \mathrm{NH}_{2}\right), 3.55$ $\left(\mathrm{m}, 2 \mathrm{H}, \mathrm{NHCH}_{2} \mathrm{CH}_{2} \mathrm{O}\right), 3.45\left(\mathrm{q}, J=5.0 \mathrm{~Hz}, 2 \mathrm{H}, \mathrm{NHCH}_{2} \mathrm{CH}_{2} \mathrm{O}\right), 2.87(\mathrm{dm}, 1 \mathrm{H}$, $\left.\mathrm{OCH}_{2} \mathrm{CH}_{2} \mathrm{NH}_{2}\right) 2.10$ (bs, 2H, $\left.\mathrm{OCH}_{2} \mathrm{CH}_{2} \mathrm{NH}_{2}\right) 2.04$ (s, 3H, $\left.\mathrm{CHCH}_{2}\right), 1.86$ (s, 6H, $\left.\mathrm{CHCH}_{2} \mathrm{CCONH}\right), 1.72\left(\mathrm{~m}, J=14.9 \mathrm{~Hz}, 6 \mathrm{H}, \mathrm{CHCH}_{2} \mathrm{CH}\right) .{ }^{13} \mathrm{C} \mathrm{NMR}\left(75 \mathrm{MHz}, \mathrm{CDCl}_{3}\right) \delta=$ $185.4,70.7,70.5,70.4,70.2,41.8,40.8,39.4,39.2,36.8,29.9,28.4,28.3$. ESI-TOF Calculated for $\mathrm{C}_{19} \mathrm{H}_{32} \mathrm{~N}_{4} \mathrm{O}_{4}$ : $m / z$ 354.3 Found: $m / z 355.3$ [M+H].

\section{Step 3: Synthesis of Ad-EG-NCS ${ }^{[17]}$ (5)}

A solution of Ad-EG-NH $\mathrm{NH}_{2}$ (4) $(30 \mathrm{mg}, 0.085 \mathrm{mmol})$ in $5 \mathrm{~mL}$ of dichloromethane was prepared in a $25 \mathrm{~mL}$ round-bottomed flask equipped with a stir bar. The solution was then cooled to $0{ }^{\circ} \mathrm{C}$ with an external ice/brine bath. Saturated aqueous $\mathrm{NaHCO}_{3}(5 \mathrm{~mL})$ was added, and the biphasic mixture stirred vigorously $(\sim 500 \mathrm{rpm})$ for $5 \mathrm{~min}$. The stirring was stopped, and thiophosgene (10 eq., $65 \mu \mathrm{L})$ was added via syringe to the organic layer. The 150 
flask was sealed with a rubber septum, and immediately, vigorous stirring was restored, the reaction was removed from the ice bath, and allowed to stir for $30 \mathrm{~min}$ at room temperature. The layers were separated and the aqueous layer was extracted with $\mathrm{CH}_{2} \mathrm{Cl}_{2}(2 \times 2 \mathrm{~mL})$. The combined organics were dried over $\mathrm{Na}_{2} \mathrm{SO}_{4}$, filtered, and concentrated in vacuo to afford the crude isothiocyanate as a yellow oil. The isothiocyanate was used in the subsequent step without any further purification. (32 $\mathrm{mg}, 0.08 \mathrm{mmol}$, 94\%). ESI-TOF Calculated for $\mathrm{C}_{20} \mathrm{H}_{32} \mathrm{~N}_{2} \mathrm{O}_{4} \mathrm{~S}: m / z$ 396.2 Found: $m / z$ 397.2 [M+H].

\section{Step 4: Synthesis of G0-PAMAM-EG-(Ad) $)_{4}$ (6)}

A solution of Ad-EG-NCS (5) (30 mg, $0.08 \mathrm{mmol}, 6$ eq) in $5 \mathrm{~mL}$ of dry $\mathrm{CHCl}_{3}$ was added to a solution of the amino-terminated G0-PAMAM dendrimer ${ }^{[18]}(0.0133 \mathrm{mmol}, 1 \mathrm{eq})$ in $5 \mathrm{~mL}$ of dry $\mathrm{CHCl}_{3}$. The reaction mixture was stirred at room temperature for $48 \mathrm{~h}$; after that time, the solvent was evaporated to afford the pure G0-PAMAM-EG-(Ad) $)_{4}(26 \mathrm{mg}, 99 \%) .{ }^{1} \mathrm{H}$ NMR (600 MHz, $\left.\mathrm{CD}_{3} \mathrm{OD}\right) \delta=3.60,3.51,3.49,3.35,3.23,2.74,2.70,2.69,2.46,2.32,2.00$, $1.79,1.70,1.66$.

${ }^{1} \mathrm{H}$ NMR (600 MHz, $\left.\left.\mathrm{CDCl}_{3}\right) \mathrm{CD}_{3} \mathrm{OD} / \mathrm{CDCl}_{3} 1 / 1 \mathrm{v} / \mathrm{v}\right) \delta=3.50(\mathrm{~m}, 32 \mathrm{H}, \mathbf{h}), 3.40(\mathrm{~m}, 8 \mathrm{H}, \mathbf{g})$, 3.27-3.13 (24H, f, i, d), $2.73(\mathrm{~m}, 8 \mathrm{H}, \mathbf{j}), 2.64(\mathrm{~m}, 8 \mathrm{H}, \mathbf{e}), 2.58(\mathrm{~m}, 8 \mathrm{H}, \mathbf{b}), 2.35(\mathrm{~s}, 4 \mathrm{H}, \mathbf{a})$, $2.22(\mathrm{~m}, 8 \mathrm{H}, \mathbf{c}), 1.89$ (bs, 12H, m), 1.69 (s, 24H, k), 1.58 (m, 24H, l). ${ }^{13} \mathrm{C}$ NMR (151 MHz, $\left.\mathrm{CD}_{3} \mathrm{OD}\right) \delta=180.9,175.3,71.5,71.3,70.5,52.3,51.2,42.4,41.8,40.1,37.6,34.6,29.6$.

\section{Method:}

\section{SAM Formation}

Ultra flat substrates of thin films of gold metal on a polymer supported by a glass slide, were obtained by delaminating an evaporated film of gold from a $\mathrm{Si} / \mathrm{SiO}_{2}$ template (the 'mechanical template-stripping' procedure (TS)) as previously described in detail. ${ }^{[13 a]} \beta C D$ SAMs were prepared by immersing freshly cleaved ultra-flat gold substrates into a 0.1-1 $\mathrm{mM}$ solution of $\beta \mathrm{CD}$ dissolved in ethanol for 16 hours at $60^{\circ} \mathrm{C}$. The substrates were removed and placed in a vial of fresh warm ethanol with the vial being gently agitated for one to two minutes. After removal they were further rinsed with ethanol (room temperature) and MilliQ water, and then dried under a stream of dry nitrogen. As previously published, Electrochemical Impedance Spectroscopy was used to characterize control $\beta C D$ SAMs and yielded similar results. 


\section{Dendrimer Absorption}

The $\beta C D$ SAM on $\mathrm{Au}$ was immersed in an aqueous solution of the corresponding dendrimer- $\beta C D$ assembly; G0-PAMAM-(Fc) $)_{4}$, G0-PAMAM-(Ad) $)_{4}$, G0-PAMAM-((EG) $)_{3}$ $\mathrm{Fc})_{4}, \mathrm{G} 0-\mathrm{PAMAM}-\left((\mathrm{EG})_{3}-\mathrm{Ad}\right)_{4}$ dendrimer, $2-3 \mathrm{mM}$ in $\mathrm{Ad} / \mathrm{Fc}$ concentration in the presence of $2-3 \mathrm{mM}$ of $\beta \mathrm{CD}$ at $\mathrm{pH}=2^{[10 \mathrm{~b}, 10 \mathrm{c}]}$ Subsequently the samples were rinsed with MilliQ water and dried under a stream of dry nitrogen. As previously published, Surface Plasmon Resonance and Cyclic Voltammetry were used to characterize the control $\beta C D$ SAM + dendrimer assemblies and yielded similar results.

\section{Calculation of Rectification Ratio}

The rectification ratio which is defined as $R=|J(-2.0 \mathrm{~V})| /|J(+2.0 \mathrm{~V})|$ was analyzed in the same fashion as the $J$ measurements for each molecular junction and as previously published. ${ }^{[3 \mathrm{~d}]}$ The $R$ for each individual scan was calculated at $\pm 2.0 \mathrm{~V}$ for each supramolecular junction structure and plotted into histograms. Fitting the histograms with single Gaussian functions gave the log-mean value (average) $R$ and the log standard deviation (with all errors stated representing one log-standard deviation, $68 \%$ of the distribution of the data is within one log-standard deviation of the log-mean, as shown in Figure 5.4b,d and 5.5b,d this Chapter and discussed in Chapter 3).

\subsection{References}

[1] A. Aviram, M. A. Ratner, Chem. Phys. Lett. 1974, 29, 277-283.

[2] a)H. Song, M. A. Reed, T. Lee, Adv. Mater. 2011, 23, 1583-1608; b)R. L. McCreery, A. J. Bergren, Adv. Mater. 2009, 21, 4303-4322; c)H. B. Akkerman, P. W. M. Blom, D. M. de Leeuw, B. de Boer, Nature 2006, 441, 69-72; d)R. Haag, M. A. Rampi, R. E. Holmlin, G. M. Whitesides, J. Am. Chem. Soc. 1999, 121, 7895-7906; e)R. C. Chiechi, E. A. Weiss, M. D. Dickey, G. M. Whitesides, Angew. Chem. Int. Ed. 2008, 47, 142-144; f)T.-W. Kim, G. Wang, H. Lee, T. Lee, Nanotechnology 2007, 18, 315204.

[3] a)C. A. Nijhuis, W. F. Reus, A. C. Siegel, G. M. Whitesides, J. Am. Chem. Soc. 2011, 133, 15397-15411; b)C. A. Nijhuis, W. F. Reus, G. M. Whitesides, J. Am. Chem. Soc. 2010, 132, 18386-18401; c)C. A. Nijhuis, W. F. Reus, J. R. Barber, M. D. Dickey, G. M. Whitesides, Nano Lett. 2010, 10, 3611-3619; d)C. A. Nijhuis, W. F. Reus, G. M. Whitesides, J. Am. Chem. Soc. 2009, 131, 17814-17827; e)S. Ramachandra, K. C. Schuermann, F. Edafe, P. Belser, C. A. Nijhuis, W. F. Reus, G. M. Whitesides, L. De Cola, Inorg. Chem. 2011, 50, 1581-1591; f)Z. Y. 
Ng, K. P. Loh, L. Q. Li, P. Ho, P. Bai, J. H. K. Yip, ACS Nano 2009, 3, 2103-2114; g)M. Elbing, R. Ochs, M. Koentopp, M. Fischer, C. von Hanisch, F. Weigend, F. Evers, H. B. Weber, M. Mayor, Proc. Natl. Acad. Sci. USA 2005, 102, 8815-8820; h)R. McCreery, J. Dieringer, A. O. Solak, B. Snyder, A. M. Nowak, W. R. McGovern, S. DuVall, J. Am. Chem. Soc. 2003, 125, 10748-10758; i)A.-S. Hallbäck, B. Poelsema, H. J. W. Zandvliet, Solid State Commun. 2007, 141, 645-648; j)M. L. Chabinyc, X. X. Chen, R. E. Holmlin, H. Jacobs, H. Skulason, C. D. Frisbie, V. Mujica, M. A. Ratner, M. A. Rampi, G. M. Whitesides, J. Am. Chem. Soc. 2002, 124, 11730-11736; k)M.-K. Ng, D.-C. Lee, L. Yu, J. Am. Chem. Soc. 2002, 124, 11862-11863; I)T. Böhme, C. D. Simpson, K. Müllen, J. P. Rabe, Chem. Eur. J. 2007, 13, 7349-7357; m)I. Dies-Perez, J. Hihath, Y. Lee, L. Yu, L. Adamska, M. A. Kozhushner, I. I. Oleynik, N. Tao, Nature Chem. 2009, 1, 635-641.

[4] a)R. M. Metzger, Anal. Chim. Acta 2006, 568, 146-155; b)G. J. Ashwell, B. Urasinska, W. D. Tyrrell, Phys. Chem. Chem. Phys. 2006, 8, 3314-3319.

[5] a)J. M. Beebe, J. G. Kushmerick, Appl. Phys. Lett. 2007, 90; b)S. Lenfant, D. Guerin, F. Tran Van, C. Chevrot, S. Palacin, J. P. Bourgoin, O. Bouloussa, F. Rondelez, D. Vuillaume, J. Phys. Chem. B 2006, 110, 13947-13958; c)R. M. Metzger, Acc. Chem. Res. 1999, 32, 950-957; d)S. Lenfant, C. Krzeminski, C. Delerue, G. Allan, D. Vuillaume, Nano Lett. 2003, 3, 741-746; e)C. N. Lau, D. R. Stewart, R. S. Williams, M. Bockrath, Nano Lett. 2004, 4, 569-572; f)V. V. Zhirnov, R. K. Cavin, Nature Mater. 2006, 5, 11-12; g)C. Zhou, M. R. Deshpande, M. A. Reed, L. Jones li, J. M. Tour, Appl. Phys. Lett. 1997, 71, 611-613.

[6] P. E. Kornilovitch, A. M. Bratkovsky, R. S. Williams, Phys. Rev. B 2002, 66, 165436.

[7] R. Liu, S. H. Ke, W. T. Yang, H. U. Baranger, J. Chem. Phys. 2006, 124, 024718.

[8] N. Armstrong, R. C. Hoft, A. McDonagh, M. B. Cortie, M. J. Ford, Nano Lett. 2007, 7, 30183022.

[9] K. S. Wimbush, W. F. Reus, W. G. van der Wiel, D. N. Reinhoudt, G. M. Whitesides, C. A. Nijhuis, A. H. Velders, Angew. Chem. Int. Ed. 2010, 49, 10176-10180.

[10] a)C. A. Nijhuis, J. Huskens, D. N. Reinhoudt, J. Am. Chem. Soc. 2004, 126, 12266-12267; b)C. A. Nijhuis, F. Yu, W. Knoll, J. Huskens, D. N. Reinhoudt, Langmuir 2005, 21, 7866-7876; c)C. A. Nijhuis, K. A. Dolatowska, B. J. Ravoo, J. Huskens, D. N. Reinhoudt, Chem. Eur. J. 2007, 13, 69-80; d)C. A. Nijhuis, B. A. Boukamp, B. J. Ravoo, J. Huskens, D. N. Reinhoudt, J. Phys. Chem. C 2007, 111, 9799-9810.

[11] E. A. Weiss, R. C. Chiechi, G. K. Kaufman, J. K. Kriebel, Z. Li, M. Duati, M. A. Rampi, G. M. Whitesides, J. Am. Chem. Soc. 2007, 129, 4336-4349. 
[12] a)M. W. J. Beulen, J. Bugler, B. Lammerink, F. A. J. Geurts, E. M. E. F. Biemond, K. G. C. van Leerdam, F. C. J. M. van Veggel, J. F. J. Engbersen, D. N. Reinhoudt, Langmuir 1998, 14, 6424-6429; b)M. W. J. Beulen, J. Bügler, M. R. de Jong, B. Lammerink, J. Huskens, H. Schönherr, G. J. Vancso, B. A. Boukamp, H. Wieder, A. Offenhäuser, W. Knol, F. C. J. M. van Veggel, D. N. Reinhoudt, Chem. Eur. J. 2000, 6, 1176-1183.

[13] a)E. A. Weiss, G. K. Kaufman, J. K. Kriebel, Z. Li, R. Schalek, G. M. Whitesides, Langmuir 2007, 23, 9686-9694; b)D. Q. Gao, F. Scholz, H. G. Nothofer, W. E. Ford, U. Scherf, J. M. Wessels, A. Yasuda, F. von Wrochem, J. Am. Chem. Soc. 2011, 133, 5921-5930.

[14] The HOMO level of the FC moieties and the change in energy of the HOMO level with applied bias were determined using the same method presented in Chapter 4 . However, for more detailed discussions the HOMO level of each individual Fc moiety may need to be treated individually, with the change in energy of the HOMO level with applied bias being dependent on the distance from the $\left(\mathrm{Ga}_{2} \mathrm{O}_{3}\right) \mathrm{EGaIN}$ top electrode

[15] J. C. Love, L. A. Estroff, J. K. Kriebel, R. G. Nuzzo, G. M. Whitesides, Chem. Rev. 2005, 105, 1103-1169.

[16] J. Huskens, M. A. Deij, D. N. Reinhoudt, Angew. Chem. Int. Ed. 2002, 41, 4467-4471.

[17] I. T. Raheem, P. S. Thiara, E. N. Jacobsen, Org. Lett. 2008, 10, 1577-1580.

[18] The amino-terminated GO-PAMAM dendrimer (Sigma-Aldrich) is supplied as $20 \% \mathrm{wt}$ solution in methanol; the complete removal of the methanol prior the coupling with the isothiocyanate is required in order to avoid the side reaction between $\mathrm{MeOH}$ and the Ad-EGNCS. 
Chapter 6

\title{
Voltage Induced Rectification in \\ EGaIn Supramolecular Tunneling
}

\author{
Junctions
}

This Chapter presents the ability to increase rectification $(R)[\mathrm{R}=|J(-2.0 \mathrm{~V})| /|J(+2.0 \mathrm{~V})|]$ in a variety of $\beta$-cyclodextrin $(\beta C D)$ Eutectic Gallium Indium $\left(\left(\mathrm{Ga}_{2} \mathrm{O}_{3}\right)\right.$ EGaIn) tunneling junctions by applying a constant 'high' positive voltage across the junction for prolonged periods of time (defined as a voltage pulse) prior to performing $J(V)$ measurements $\pm 2.0 \mathrm{~V}$. The resulting $R$ is dependent on the applied voltage and length of time of the pulse. 


\subsection{Introduction}

For the field of molecular electronics to continue to progress, it is essential to have a clear fundamental understanding of charge transport across the entire molecular tunneling junction, which includes two electrodes, two interfaces and a molecular intermediate. An 'ideal' technique is still not available to investigate all components and interfaces, however, in recent years several methodologies have been developed allowing for a greater understanding of charge transport across molecular tunneling junctions. ${ }^{[1]}$ One technique is the $\left(\mathrm{Ga}_{2} \mathrm{O}_{3}\right)$ EGaIn top-electrode technique developed by Whitesides and co-workers. ${ }^{[\mathrm{b}]}$ This technique has been able to form stable molecular tunneling junctions (stable for up to 20 hours) thus allowing statistical amounts of reproducible data to be collected ( $>400 \mathrm{~J}(\mathrm{~V})$ scans) with high yields of working junctions $(80-100 \%){ }^{[2]}$ The $\left(\mathrm{Ga}_{2} \mathrm{O}_{3}\right)$ EGaIn technique, like all other techniques also has its disadvantages. The thin layer of $\mathrm{Ga}_{2} \mathrm{O}_{3}$ on the surface of the EGaIn adds complexity to the interpretation of the data obtained from $\left(\mathrm{Ga}_{2} \mathrm{O}_{3}\right) \mathrm{EGaIn}$ tunneling junctions.

Since its conception, the EGaIn technique has allowed for an extensive investigation of charge transport by applying direct current $(\mathrm{DC})^{[1 \mathrm{~b}, 2-3]}$ through various kinds of Self Assembled Monolayers (SAMs), from alkanethiolates, ${ }^{\left[1 \mathrm{~b},{ }^{2]}\right.}$ thiolates of oligo (phenylene ethynylenes), ${ }^{[3 \mathrm{a}]}$ terphenylenes and arylethynylenes ${ }^{[3 \mathrm{~b}]}$ to SAMs that rectify such as ferrocene $(\mathrm{Fc})$ functionalized alkanethiolates, ${ }^{[3 \mathrm{c}-\mathrm{e}]}$ and supramolecular tunneling junctions comprised of $\mathrm{Fc}$ terminated dendrimers immobilized on a heptathioether functionalized $\beta$-cyclodextrin SAM ( $\beta$ CD SAM) ${ }^{[4]}$ One influential difference between the supramolecular assemblies $^{[4]}$ and the molecular assemblies investigated ${ }^{[1 b, 2-3]}$ using $\left(\mathrm{Ga}_{2} \mathrm{O}_{3}\right)$ EGaIn is the thickness of the molecular layer. The thickness of the $\beta C D$ SAM is approximately $2.5 \mathrm{~nm}$, which is comparable to a $\mathrm{C} 16-\mathrm{C} 18$ alkanethiolate $\mathrm{SAM} .^{[5]}$ Upon the addition of the dendrimer layer to the $\beta C D$ SAM the thickness of the molecular layer increases to approximately 2.75-3 $\mathrm{nm}$ (depending on the dendrimer absorbed). This clearly affects the breakdown voltage, (i.e. voltage at which an ohmic response is obtained) which can also be defined as the breakdown field, with $E=V / d(E=$ Electric field, $V=$ voltage, $d=$ distance between the electrodes/thickness of the molecular layer of the molecular (in meters) system) when performing $\mathrm{DC}$ measurements $\approx 3.5 \mathrm{~V}(\mathrm{E} \approx 1.2 \mathrm{GV} / \mathrm{m}-1.4 \mathrm{GV} / \mathrm{m})$ for the 
supramolecular system vs. $\approx 1.0$ to $1.5 \mathrm{~V}(\mathrm{E} \approx 400 \mathrm{MV} / \mathrm{m}-600 \mathrm{MV} / \mathrm{m})$ for all other studied molecular assemblies). ${ }^{[1 \mathrm{~b}, 2-4]}$

Due to the unique ability of the supramolecular tunneling junctions to withstand such relatively large voltages, hence, electric fields, it was possible to investigate the effects that large applied biases can have on EGaIn tunneling junctions. It was discovered that prolonged cycling of the voltage (i.e. 100 voltage scans $\pm 2.0 \mathrm{~V}$ ) or applying voltage pulses (maximum $+2.5 \mathrm{~V}$ ) for up to 1 hour prior to performing $J(V)$ measurements $\pm 2.0 \mathrm{~V}$, changed the $J(V)$ characteristics, resulting in a change of $R$ (where $R=|J(-2.0 \mathrm{~V})| / \mid J(+2.0 \mathrm{~V} \mid)$ ) of up to five orders of magnitude. Interestingly, this trend is consistent for all supramolecular assemblies investigated. Through experimental characterization of all components and interfaces of the tunneling junction, it is now suggested that the origin of the increase in $R$ is a change in the $\mathrm{Ga}_{2} \mathrm{O}_{3}$ layer on the EGaIn as a consequence of the high voltage applied, and not due to the molecules themselves.

\subsection{Construction of the Supramolecular Tunneling Junctions: Generation One Poly(propylene) imine Dendrimers and Generation Zero Poly(amido amine) Dendrimers}

Figure 6.1 on page 159, shows the supramolecular platform used in this investigation, a well-defined hexagonally packed SAM of heptathioether-functionalized $\beta C D,{ }^{[6]}$ formed on a $\mathrm{Au}$ template stripped surface $\left(\mathrm{Au}^{\mathrm{TS}}\right)$. Along with the bare $\beta C D$ SAM tunneling junction an additional four different tunneling junctions were prepared by adsorbing dendrimers on the $\beta C D$ monolayer through host-guest interactions. The four different dendrimers used were: generation-1 poly(propylene) dendrimers molecules with ferrocene (G1-PPI-(Fc) 4 ) or adamantyl (G1-PPI-(Ad) $)_{4}$ ) functional end groups, and generation-0 poly(amido amine) dendrimers with adamantyl (G0-PAMAM- $\left.(\mathrm{Ad})_{4}\right)$ or ethylene glycol tether with adamantyl (G0-PAMAM-((EG) $\left.\left.)_{3}-\mathrm{Ad}\right)_{4}\right)$. All four dendrimers used have four functional end groups, but their interaction with the $\beta C D$ monolayer is very different. The G1-PPI dendrimers adsorb by divalent interactions of two of the end groups with two of the $\beta \mathrm{CD}$ molecules on the surface, leaving two end groups non-interacting with the surface (Figure 6.1 on page 159, Table 6.1 on page 160), according to reference ${ }^{[7]}$. The PAMAM dendrimers interact differently, with the adamantyl-functionalized dendrimer having trivalent interactions with 
the $\beta C D$ platform, and the ethylene-glycol-adamantyl dendrimer having a tetravalent interaction with the surface. (Figure 6.1 on page 159, Table 6.1 on page 160). ${ }^{[7]}$

Figure 6.1(page 159): Idealized schematic of the supramolecular tunneling junctions, with the metalmolecule-metal interfaces emphasized as phenomena is expected to occur in these areas when large voltages are applied across the junctions. All junctions are described in the text using nomenclature, $\mathrm{Au}^{\mathrm{TS}}$ $\beta C D S A M / X / /\left(\mathrm{Ga}_{2} \mathrm{O}_{3}\right) \mathrm{EGaIn}$ where "-." in $\mathrm{Au}^{\mathrm{TS}}-\beta \mathrm{CD}$ represents the non-covalent interface of the Au surface and the sulfur of the heptathioether functionalized $\beta \mathrm{CD}$, "/" represents the supramolecular host-guest interaction between the $\beta C D$ and the terminal functional group of the dendrimer, "X" represents the dendrimer, " $\left(\mathrm{Ga}_{2} \mathrm{O}_{3}\right)$ " represents the oxides of gallium present on the skin of the EGaIn and "//"represents the Van der Waals interactions at the interface between the terminal group of the molecular structure and the $\mathrm{Ga}_{2} \mathrm{O}_{3}$ on the

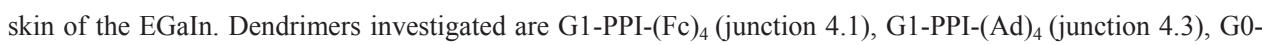
PAMAM-(Ad) $)_{4}$ (junction 5.2) and G0-PAMAM-((EG) $\left.)_{3}-\mathrm{Ad}\right)_{4}$ (junction 5.4). Additionally, the bare $\beta C D$ SAM (supramolecular platform) (junction 4.4) was also investigated in this study. 

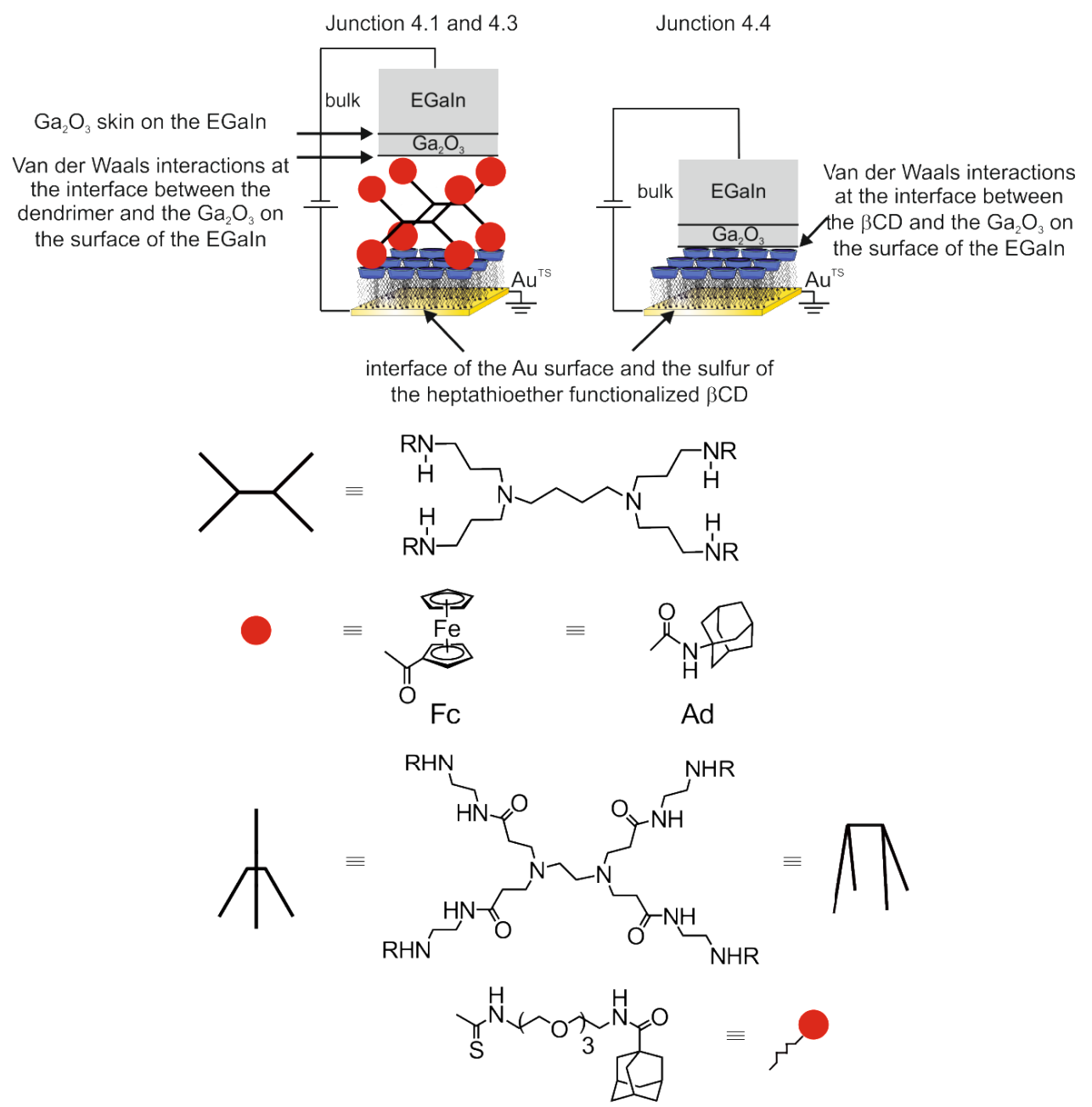

ethylene glycol-Ad

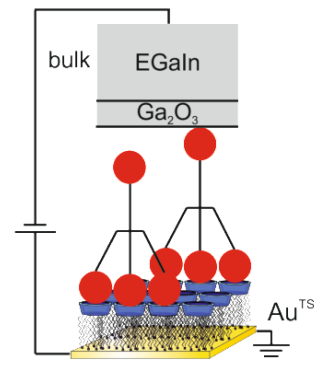

Junction 5.2

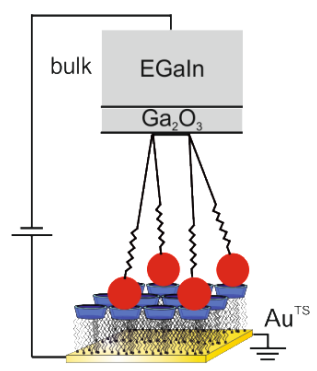

Junction 5.4 
The top contact was applied using the $\left(\mathrm{Ga}_{2} \mathrm{O}_{3}\right)$ EGaIn technique ${ }^{[4]}$. This gave five supramolecular tunneling junctions, $\mathrm{Au}^{\mathrm{TS}}-\beta \mathrm{CDSAM} / \mathrm{G} 1-\mathrm{PPI}-(\mathrm{Fc})_{4} / /\left(\mathrm{Ga}_{2} \mathrm{O}_{3}\right) \mathrm{EGaIn}$ (4.1), $\mathrm{Au}^{\mathrm{TS}}-\beta \mathrm{CDSAM} / \mathrm{G} 1-\mathrm{PPI}-(\mathrm{Ad})_{4} / /\left(\mathrm{Ga}_{2} \mathrm{O}_{3}\right)$ EGaIn (4.3), $\quad \mathrm{Au}^{\mathrm{TS}}-\beta C D S A M / G 0-P A M A M-$ $(\mathrm{Ad})_{4} / /\left(\mathrm{Ga}_{2} \mathrm{O}_{3}\right)$ EGaIn (5.2), $\mathrm{Au}^{\mathrm{TS}}-\beta \mathrm{CDSAM} / \mathrm{G} 0-\mathrm{PAMAM}-\left((\mathrm{EG})_{3}-\mathrm{Ad}\right)_{4} / /\left(\mathrm{Ga}_{2} \mathrm{O}_{3}\right)$ EGaIn (5.4) and $\mathrm{Au}^{\mathrm{TS}}-\beta \mathrm{CDSAM} / /\left(\mathrm{Ga}_{2} \mathrm{O}_{3}\right) \mathrm{EGaIn}$ (4.4). To allow for a consistent representation of the effect that applying large biases has on the EGaIn supramolecular tunneling junctions, a large majority of the data presented in this Chapter is from experiments performed on junction 4.1. Junctions 4.3 and 4.4 serve as control experiments in section 6.3 .5 and junctions 5.2 and 5.4 are used in section 6.3 .6 to investigate the cause/origin of the increase in $R$.

Table 6.1: Dendrimer adsorption properties on the $\beta$ CD SAM (supramolecular platform)

\begin{tabular}{|c|c|c|c|}
\hline Molecular Junction $^{[\mathrm{a}]}$ & $\begin{array}{l}\text { No. of interactions } \\
\text { with } \beta \text { CD SAM }{ }^{[b]}\end{array}$ & $\begin{array}{l}\text { No. of interactions } \\
\text { with }\left(\mathrm{Ga}_{2} \mathrm{O}_{3}\right) \text { EGaIn } \\
\text { top electrode }{ }^{[\mathrm{c}]}\end{array}$ & $\begin{array}{l}\text { Surface Coverage of } \\
\beta C D \text { SAM }(\%)^{[d]}\end{array}$ \\
\hline 4.1 "PPI-Fc" & 2 & 2 & $\sim 89$ \\
\hline 4.3 "PPI-Ad" & 2 & 2 & $>95$ \\
\hline 5.2 "PAMAM -Ad" & 3 & 1 & $\sim 100$ \\
\hline 5.4 "PAMAM-((EG) $\left.)_{3}-\mathrm{Ad}\right)_{4} "$ & 4 & 0 & $\sim 100$ \\
\hline 4.4 " $\beta$ CD SAM" & - & - & - \\
\hline
\end{tabular}

[a] $4.1-\mathrm{Au}^{\mathrm{TS}}-\beta C D S A M / G 1-P P I-(\mathrm{Fc})_{4} /\left(\mathrm{Ga}_{2} \mathrm{O}_{3}\right)$ EGaIn, $4.3-\mathrm{Au}^{\mathrm{TS}}-\beta C D S A M / G 1-P P I-(\mathrm{Ad})_{4} / /\left(\mathrm{Ga}_{2} \mathrm{O}_{3}\right) \mathrm{EGaIn}$, $5.2=\mathrm{Au}^{\mathrm{TS}}-\beta \mathrm{CDSAM} / \mathrm{G} 1-\mathrm{PAMAM}-(\mathrm{Ad})_{4} / /\left(\mathrm{Ga}_{2} \mathrm{O}_{3}\right)$ EGaIn, $\quad 5.4=\mathrm{Au}^{\mathrm{TS}}-\beta C D S A M / \mathrm{G} 1-\mathrm{PPI}-\left((\mathrm{EG})_{3^{-}}\right.$ $\mathrm{Ad})_{4} / /\left(\mathrm{Ga}_{2} \mathrm{O}_{3}\right)$ EGaIn, $4.4=\mathrm{Au}^{\mathrm{TS}}-\beta \mathrm{CDSAM} / /\left(\mathrm{Ga}_{2} \mathrm{O}_{3}\right)$ EGaIn. [b] = amount of dendrimer terminal moieties that form host-guest interactions with the $\beta C D$ SAM, out of a possible total of four. [c] = amount of dendrimer terminal moieties coupled with the $\left(\mathrm{Ga}_{2} \mathrm{O}_{3}\right)$ EGaIn top electrode, out of a possible of four. [d] surface coverage $(\%)$ of the dendrimer adsorbed to the supramolecular platform. ${ }^{[7]}$ 


\subsection{Results and Discussion}

This section describes how $R$ (defined as $R=|J(-2.0 \mathrm{~V})| /|J(+2.0 \mathrm{~V})|$ ) in supramolecular tunneling junctions changes upon prolonged, cyclic scanning (i.e. 100 scans) $\pm 2.0 \mathrm{~V}$. Additionally, it is presented that this change in $R$ can also be achieved by applying a voltage pulse of $+2.0 \mathrm{~V}(E \approx 666 \mathrm{MV} / \mathrm{m})$ across the junction for a specified period of time, by comparing $J(V) \mathrm{scan} / \mathrm{s} \pm 2.0 \mathrm{~V}$, performed before and after the voltage pulse. Furthermore, by increasing the voltage pulse to $+2.5 \mathrm{~V}(E \approx 833 \mathrm{MV} / \mathrm{m})$ it is shown that the $R$ measured in the preceding $J(V)$ scans $\pm 2.0 \mathrm{~V}$ is greater than that of $R$ measured in the $J(V)$ scans \pm 2.0 $\mathrm{V}$ performed after the $+2.0 \mathrm{~V}$ pulse. Also demonstrated is how $R$ depends on the voltage and duration of the pulse. In the last section, the investigation of the origin of the increase of $R$ is discussed.

\subsubsection{Prolonged Cyclic $J(V)$ Scanning $\pm 2.0 \mathrm{~V}$}

To test the stability of junction 4.1, $100 J(V)$ scans were performed $\pm 2.0 \mathrm{~V}$. From the measurements an unexpected phenomenon was observed. Figure 6.2a displays the 100 $|J|(V)$ scans $\pm 2.0 \mathrm{~V}$, with each color set representing a block of 20 scans within the 100 scans. When comparing each block of 20 scans it can be seen that as the scan set increases, the value of $|J|$ measured at $-2.0 \mathrm{~V}$ increases and at $+2.0 \mathrm{~V}$ decreases. By plotting $|J|$ measured at $-2.0 \mathrm{~V}$ and $+2.0 \mathrm{~V}$ as a function of scan number it is shown that over the course of the 100 scans $|J|$ measured at $-2.0 \mathrm{~V}$ increases by approximately one order of magnitude and at $+2.0 \mathrm{~V}$ decreases by approximately one order of magnitude (Figure $6.2 \mathrm{~b}$ ). Therefore, this increases the value of $R$ measured from 1.2 at scan 1 , to $2.4 \times 10^{2}$ by scan 100 (Figure 6.2c). For results obtained from the same measurements performed on junctions 4.3 and 4.4 , refer to appendix, section A.6.1. 

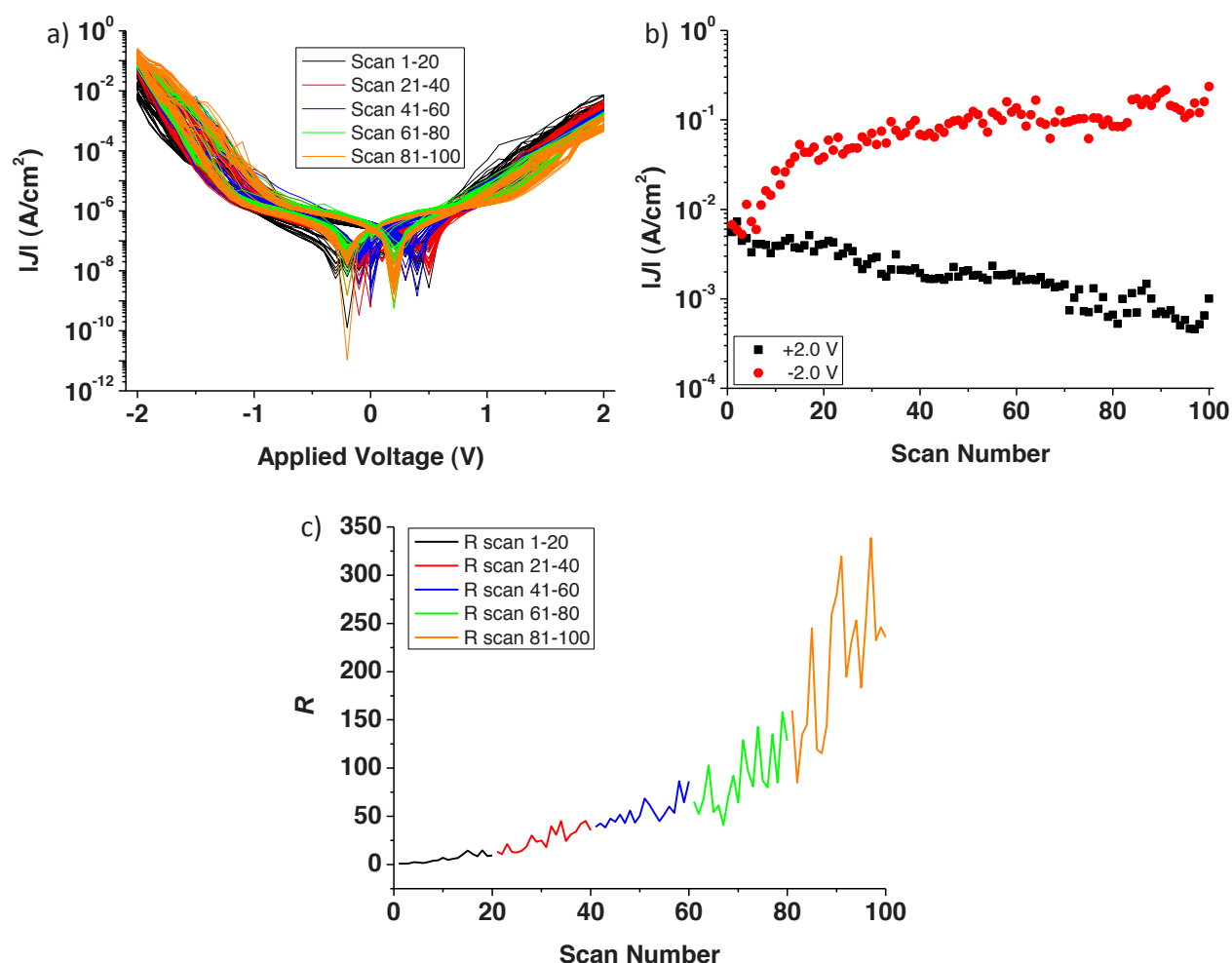

Figure 6.2: $J(V)$ measurements performed on the supramolecular junction $\mathrm{Au}^{\mathrm{TS}}-\beta C D S A M / \mathrm{G} 1-\mathrm{PPI}-$ $(\mathrm{Fc})_{4} /\left(\mathrm{Ga}_{2} \mathrm{O}_{3}\right)$ EGaIn (4.1): (a) Semi-log plot of $100|J|(V)$ scans measured $\pm 2.0 \mathrm{~V}$, Scan 1-20 (black), Scan 2140 (red), Scan 41-60 (blue), Scan 61-80 (green), Scan 81-100 (yellow), (b) semi-log plot of $|J|$ measured at -2.0 $\mathrm{V}$ and $+2.0 \mathrm{~V}$ vs. number of scans performed, (c) rectification calculated from scans shown in Figure $2 \mathrm{a}$ $[\mathrm{R}=|J(-2.0 \mathrm{~V})| /|J(+2.0 \mathrm{~V})|]$ vs. number of scans performed.

\subsubsection{Voltage Pulses $-2.0 \mathrm{~V}$ and $+2.0 \mathrm{~V}$}

To investigate which applied bias range is causing the change in $|J|$ measured and thus increasing the value of $R$ in junction 4.1, one initial $J(V)$ scan was performed $\pm 2.0 \mathrm{~V}$ followed by a $15 \mathrm{~min}$ voltage pulse of either $+2.0 \mathrm{~V}$ or $-2.0 \mathrm{~V}$ (the outermost voltages measured in the $J(V)$ scans). After the voltage pulse, five $J(V)$ scans were performed $\pm 2.0 \mathrm{~V}$, and the results of these $J(V)$ scans were compared to the initial scan. Figure 6.3a (page 164) displays two sets of $|J|(V)$ curves measured $\pm 2.0 \mathrm{~V}$; in black, the initial $|J|(V)$ scan performed prior to a voltage pulse of $+2.0 \mathrm{~V}$, in red, five $|J|(V)$ scans performed after the voltage pulse of $+2.0 \mathrm{~V}$. Figure $6.3 \mathrm{~b}$ (page 164) shows what happens to the $|J|$ measured whilst the voltage 162 
pulse of $+2.0 \mathrm{~V}$ is being applied. Similarly to the results obtained during the cyclic scanning, the measured $R$ value increased after the voltage pulse of $+2.0 \mathrm{~V}$. The initial scan (black scan) $\pm 2.0 \mathrm{~V}$ gave a value of $|J|$ of $3.0 \times 10^{-2} \mathrm{~A} / \mathrm{cm}^{2}$ and $9.1 \times 10^{-2} \mathrm{~A} / \mathrm{cm}^{2}$ at $-2.0 \mathrm{~V}$ and +2.0 $\mathrm{V}$, respectively, giving a value of $R$ of 0.3 . The five $J(V)$ scans performed (red scans) $\pm 2.0 \mathrm{~V}$ after the voltage pulse, gave an average value of $|J|$ of $9.3 \times 10^{-1} \mathrm{~A} / \mathrm{cm}^{2}$ and $8.6 \times 10^{-3} \mathrm{~A} / \mathrm{cm}^{2}$ at $-2.0 \mathrm{~V}$ and $+2.0 \mathrm{~V}$, respectively, giving an average value of $R$ of $1.1 \times 10^{2}$ (Figure 6.3a). Therefore, $R$ increased by approximately three orders of magnitude by applying the voltage pulse. Interestingly, by monitoring the value of $J$ as a function of time whilst applying the voltage pulse of $+2.0 \mathrm{~V}$ (Figure $6.2 \mathrm{~b}$ ), it can be seen that the value of $J$ measured at Time $(t)$ $=0 \min \left(J=8.0 \times 10^{-1} \mathrm{~A} / \mathrm{cm}^{2}\right)$ is similar to that of $J$ measured at $+2.0 \mathrm{~V}$ $\left(J=9.1 \times 10^{-1} \mathrm{~A} / \mathrm{cm}^{2}\right)$ in the initial $J(V)$ scan performed before applying the voltage pulse (black scan). During the measurement, $J$ slowly decreases for approximately two and a half minutes, and then continues to decrease at an even slower rate until the end of the fifteen minute measurement. $J$ measured at $t=15 \min \left(J=2.8 \times 10^{-1} \mathrm{~A} / \mathrm{cm}^{2}\right)$ is approximately the same as $J$ measured at $+2.0 \mathrm{~V}\left(J=2.4 \times 10^{-1} \mathrm{~A} / \mathrm{cm}^{2}\right)$ in the first subsequent $J(V)$ scan $\left(1^{\text {st }}\right.$ red scan) after the voltage pulse. ${ }^{[8]}$

In contrast, after applying the voltage pulse of $-2.0 \mathrm{~V}$, there was not a substantial increase in the value of $R$. Figure $6.3 \mathrm{c}$ displays two sets of $|J|(V)$ curves measured $\pm 2.0 \mathrm{~V}$; in black, the initial $|J|(V)$ scan performed prior to the voltage pulse of $-2.0 \mathrm{~V}$, in red, five $|J|(V)$ scans performed after the voltage had been applied. Figure $6.3 \mathrm{~d}$ shows what happens to $|J|$ measured whilst the voltage pulse of $-2.0 \mathrm{~V}$ was being applied. The initial scan (black scan) $\pm 2.0 \mathrm{~V}$ gave a value of $|J|$ of $6.0 \times 10^{-3} \mathrm{~A} / \mathrm{cm}^{2}$ and $7.2 \times 10^{-3} \mathrm{~A} / \mathrm{cm}^{2}$ at $-2.0 \mathrm{~V}$ and $+2.0 \mathrm{~V}$, respectively, giving a value of $R$ of 0.8 . The five $J(V)$ scans measured (red scans) $\pm 2.0 \mathrm{~V}$ after the voltage pulse had been applied, gave an average value of $|J|$ of $9.4 \times 10^{-1} \mathrm{~A} / \mathrm{cm}^{2}$ and $2.2 \times 10^{-3} \mathrm{~A} / \mathrm{cm}^{2}$ at $-2.0 \mathrm{~V}$ and $+2.0 \mathrm{~V}$, respectively, giving an average value of $R$ of 9.4 (Figure 6.3c). The value of $R$ of 9.4 is much smaller than $R$ found after applying the voltage pulse of $+2.0 \mathrm{~V}$ across the junction, with $R$ in that case being $1.1 \times 10^{2}$ (Figure 6.3a). Therefore, it is suggested that the small increase in the value of $R$ is attributed to the $|J|(V)$ scans performed $\pm 2.0 \mathrm{~V}$ in order to calculate $R$, and not the voltage pulse of $-2.0 \mathrm{~V}$ itself. Additionally, from Figure $6.3 \mathrm{~d}$ it can be seen that $J$ did not significantly decrease during the application of the voltage pulse. Hence, the results obtained from applying voltage pulses of $+2.0 \mathrm{~V}$ and $-2.0 \mathrm{~V}$ across junction 4.1 indicate that a positive applied bias is causing the $|J|$ 
measured to change, thus increasing the value of $R$ measured across the junction, and not a negative applied bias.
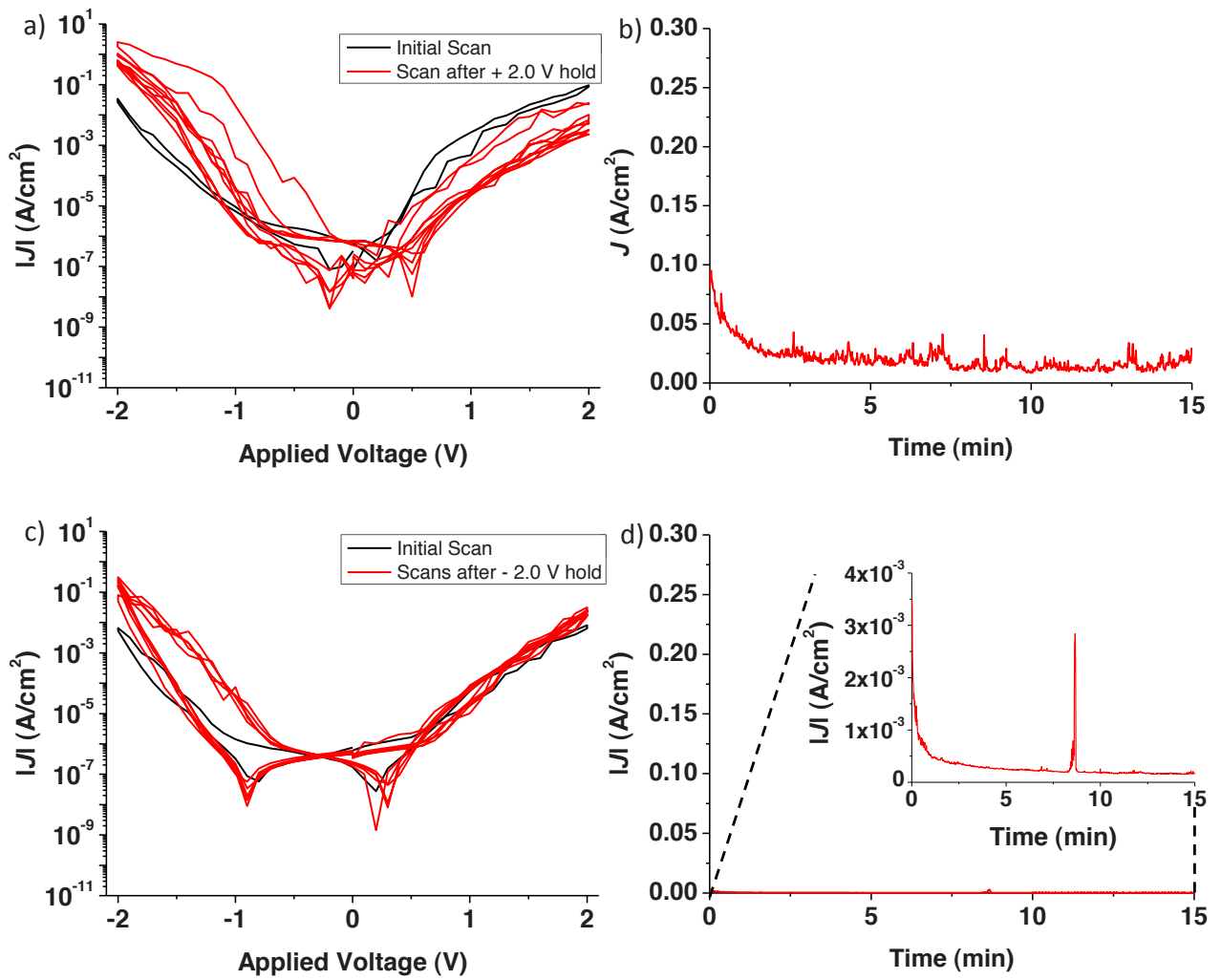

Figure 6.3: $J(V)$ measurements performed on the supramolecular junction $\mathrm{Au}^{\mathrm{TS}}-\beta C D S A M / G 1-P P I-$ $(\mathrm{Fc})_{4} / /\left(\mathrm{Ga}_{2} \mathrm{O}_{3}\right)$ EGaIn (4.1). (a) Semi-log plot of a $|J|(V)$ scan $\pm 2.0 \mathrm{~V}$ before a voltage pulse of $+2.0 \mathrm{~V}$ was applied to the junction (black scan) and five $|J|(V)$ scans measured $\pm 2.0 \mathrm{~V}$ after the voltage pulse was applied to the junction (red scans). (b) $|J|$ measured whilst the voltage pulse was being applied at $+2.0 \mathrm{~V}$ for $15 \mathrm{~min} \cdot{ }^{[8]}$ (c) Semi-log plot of a $|J|(V)$ scan $\pm 2.0 \mathrm{~V}$ before the junction was exposed to voltage pulse of $-2.0 \mathrm{~V}$ (black scan) and five $|J|(V)$ scans measured $\pm 2.0 \mathrm{~V}$ after the voltage pulse was applied (red scans). (d) $|J|$ measured whilst the voltage pulse was being applied at $-2.0 \mathrm{~V}$ for $15 \mathrm{~min}$. 


\subsubsection{Voltage Pulse $+2.5 \mathrm{~V}$}

To further investigate the increase of $R$ at a positive applied bias, a 'fresh' junction 4.1 was formed and the voltage pulse applied for $15 \mathrm{~min}$ was increased to $+2.5 \mathrm{~V}$. Figure $6.4 \mathrm{a}$ displays two sets of $|J|(V)$ curves measured $\pm 2.0 \mathrm{~V}$; in black, the initial $|J|(V)$ scan performed prior to the voltage pulse of $+2.5 \mathrm{~V}$, in red, five $|J|(V)$ scans performed after the $+2.5 \mathrm{~V}$ pulse was applied. Figure $6.4 \mathrm{~b}$ shows what happens to $J$, as a function of time, whilst the voltage pulse was being applied. The initial scan (black scan) $\pm 2.0 \mathrm{~V}$ gave a value of $|J|$ of $5.8 \times 10^{-2}$ $\mathrm{A} / \mathrm{cm}^{2}$ and $4.8 \times 10^{-2} \mathrm{~A} / \mathrm{cm}^{2}$ at $-2.0 \mathrm{~V}$ and $+2.0 \mathrm{~V}$, respectively, giving a value of $R$ of 1.2 (Figure 6.4a). The five $J(V)$ scans measured (red scans) $\pm 2.0 \mathrm{~V}$ after the voltage pules of $+2.5 \mathrm{~V}$, gave an average value of $|J|$ of $3.6 \times 10^{-5} \mathrm{~A} / \mathrm{cm}^{2}$ and $3.2 \times 10^{-1} \mathrm{~A} / \mathrm{cm}^{2}$ at $-2.0 \mathrm{~V}$ and $+2.0 \mathrm{~V}$, respectively, giving an average value of $R$ of $8.8 \times 10^{3}$ (Figure 6.4a). When monitoring $|J|$ as a function of time during the voltage pulse of $+2.5 \mathrm{~V},|J|$ decreased in a similar fashion to that found for the voltage pulse of $+2.0 \mathrm{~V}$. At $t=0 \mathrm{~min},|J|=2.7 \times 10^{-1}$ $\mathrm{A} / \mathrm{cm}^{2}$ and decreased very quickly up to the 1-1.25 minute mark and then continued to decrease slowly for the entire fifteen minute period, with $|J|=1.7 \times 10^{-3} \mathrm{~A} / \mathrm{cm}^{2}$ at $t=15 \mathrm{~min}$ (Figure 6.4b). When directly comparing these results to the results obtained when applying a voltage pulse of $+2.0 \mathrm{~V}$, it can be seen that the value of $R$ measured is nearly two orders of magnitude larger $\left(8.8 \times 10^{3}\right.$ vs. $\left.1.1 \times 10^{2}\right)$, the majority of the decrease in $|J|$ occurs faster $(1$ $1.25 \mathrm{~min}$ vs. $2.5 \mathrm{~min}$ ), and $|J|$ also decreases a larger amount (two orders of magnitude vs. half an order of magnitude). Therefore, the larger the bias applied during the pulse administered across the supramolecular junction for a specified period of time, the larger the change in $|J|$ and thus the larger value of $R$ obtained when consecutively performing $J(V)$ scans. Voltage pulses were also applied at larger biases, such as $+3.0 \mathrm{~V}$, however, the junctions were less stable, and broke down. 

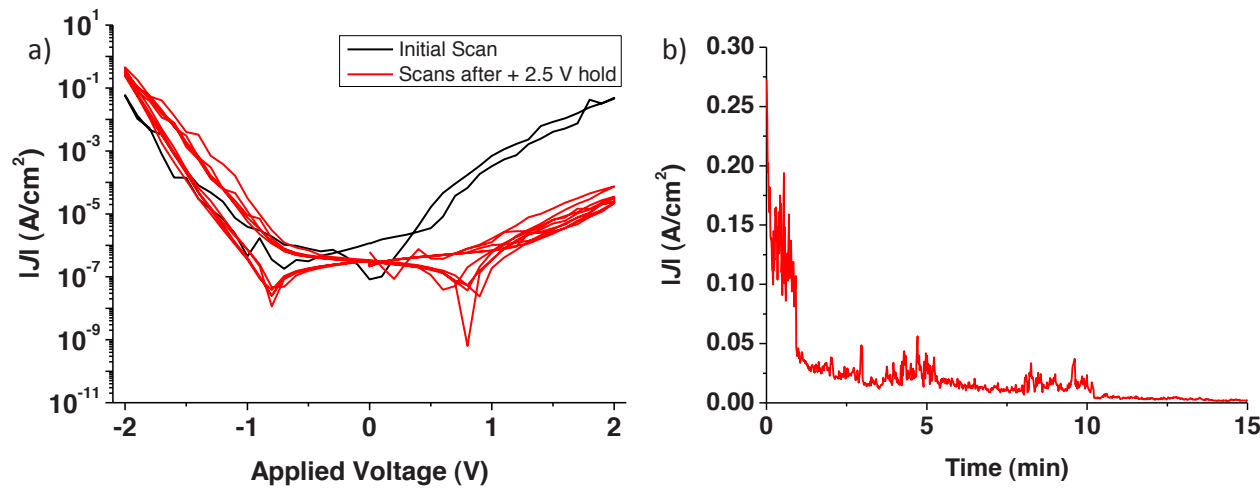

Figure 6.4: $J(V)$ Measurements performed on the supramolecular junction $\mathrm{Au}^{\mathrm{TS}}-\beta C D S A M / \mathrm{G} 1-\mathrm{PPI}-$ $(\mathrm{Fc})_{4} / /\left(\mathrm{Ga}_{2} \mathrm{O}_{3}\right)$ EGaIn (4.1); (a) Semi-log plot of a $|J|(V)$ scan measured $\pm 2.0 \mathrm{~V}$ before a pulse of $+2.5 \mathrm{~V}$ was applied to the junction (black scan) and five $|J|(V)$ scans measured $\pm 2.0 \mathrm{~V}$ after a pulse of $+2.5 \mathrm{~V}$ was applied to the junction (red scans), (b) $|J|$ measured whilst the pulse was being applied at $+2.5 \mathrm{~V}$ for $15 \mathrm{~min}^{\left[{ }^{[8]}\right.}$

\subsubsection{Rectification Measured Vs. Various Voltage Pulses}

To determine the effect that a variety of voltage pulses (different voltages applied over different periods of time), can have on the value of $R$ obtained, voltage pulses of $+1.0 \mathrm{~V}$, $+1.5 \mathrm{~V},+2.0 \mathrm{~V}$ and $+2.5 \mathrm{~V}$ were applied on separate junctions (junction 4.1) (Figure 6.5). After four representative $|J|(V)$ scans $\pm 2.0 \mathrm{~V}$ to determine $R$ at $(t)=0 \mathrm{~min}$, the voltage pulse was applied for an accumulative time of $60 \mathrm{~min}$, with $R$ being calculated after the pulse had been applied for $2,5,10,15,30,45$ and $60 \mathrm{~min}$, by performing five $|J|(V)$ scans $\pm 2.0 \mathrm{~V}$. The amount of scans chosen to check $R$ is important as accumulative $|J|(V)$ scans $\pm 2.0 \mathrm{~V}$ also increase the value of $R$, vide supra, but five scans allowed for an acceptable average value of $R$ to be calculated, whilst minimizing the effect it has on the accumulate value of $R$. It is also highly important to have a stable junction, as it takes more than two hours to complete one full measurement.

Figure 6.5a displays the value of $R$ measured after the voltage pulses were applied across junction 4.1. The larger the voltage applied and the longer the duration of the pulse, the higher the accumulative value of $R$ obtained, which agrees with the results reported above in this Chapter. This trend is already clearly seen after the voltage pulse has been applied for an accumulative time of 5-10 min, and all of the accumulative values of $R$ start to reach their 166 
maximum after the voltage pulse has been applied for 15-30 min. The values of $R$ obtained after applying pulses at $+2.5 \mathrm{~V}$ and $+2.0 \mathrm{~V}$ for 60 min are remarkably high, with $R$ being $3.3 \times 10^{4}$ and $4.2 \times 10^{3}$, respectively (Figure $6.5 \mathrm{a}$ ), which is $\approx 10^{3}$ higher than $R$ measured for other ferrocene terminated molecular tunneling junctions, i.e. $1.7 \times 10^{2}\left(\sigma_{\log }=1.9\right)$ for measurements of the junction $\mathrm{Au}^{\mathrm{TS}}-\beta \mathrm{CDSAM} / \mathrm{G} 1-\mathrm{PPI}-(\mathrm{BFc})_{4} / /\left(\mathrm{Ga}_{2} \mathrm{O}_{3}\right) \mathrm{EGaIn} \pm 2.0 \mathrm{~V}$ (Chapter 4), and $1.0 \times 10^{2}\left(\sigma_{\log }=3.0\right) \pm 1.0 \mathrm{~V}$ for $\mathrm{Fc}$ terminated alkanethiolates. Also, the value of $R$ seems to increase exponentially as a function of the applied voltage pulse (Figure 6.5b) from 49 for a 60 min pulse of $+1.0 \mathrm{~V}$ to $4.2 \times 10^{3}$ for a 60 min pulse of $+2.5 \mathrm{~V}$.
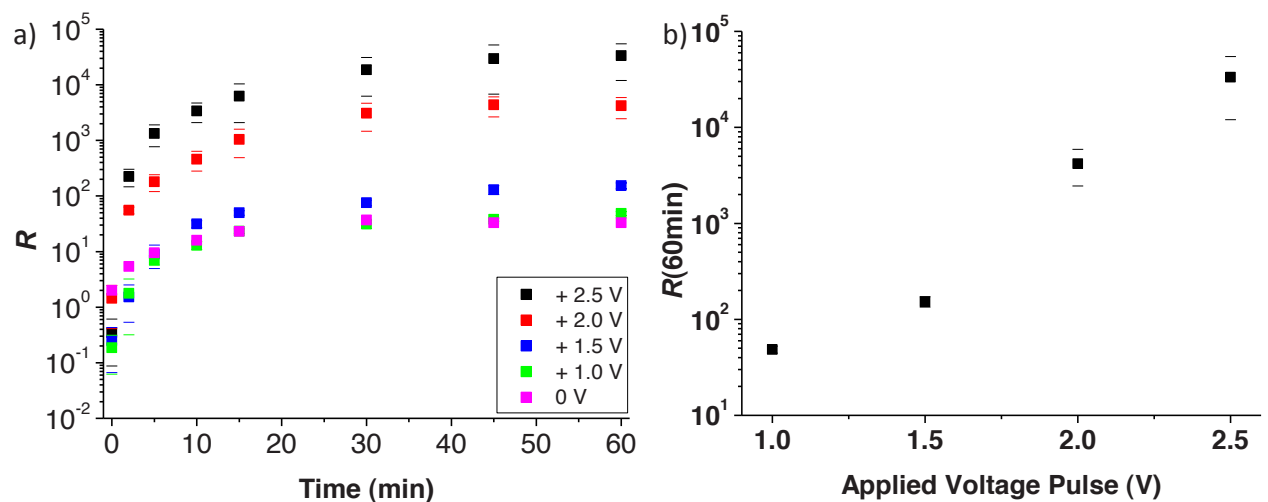

Figure 6.5: (a) Semi-log plot of the increase in rectification $(R)[\mathrm{R}=|J(-2.0 \mathrm{~V})| /|J(+2.0 \mathrm{~V})|]$ supramolecular junctions of $\mathrm{Au}^{\mathrm{TS}}-\beta \mathrm{CDSAM} / \mathrm{G} 1$-PPI-( $\left.\mathrm{Fc}\right)_{4} /\left(\mathrm{Ga}_{2} \mathrm{O}_{3}\right)$ EGaIn (4.1) when applying a pulse of $+2.5 \mathrm{~V}$ (black), +2.0 $\mathrm{V}$ (red), $+1.5 \mathrm{~V}$ (blue), $+1.0 \mathrm{~V}$ (green) over an accumulative time of $60 \mathrm{~min}$. After four representative $J(V)$ scans $\pm 2.0 \mathrm{~V}$ to determine $R$ at time $(t)=0 \mathrm{~min}, R$ is calculated by performing five $J(V)$ scans $\pm 2.0 \mathrm{~V}$ after the accumulative pulse time of 2, 5, 10, 15, 30, 45 and 60 min. Included within the plot is $R$ calculated when no voltage pulse is applied across the junction (hence ' $0 \mathrm{~V}$ ') and only accumulative $J(V)$ scans are performed \pm 2.0 $\mathrm{V}$ (as in Figure 6.2c). The amount of accumulative $J(V)$ scans performed $\pm 2.0 \mathrm{~V}$ correlate directly with the total amount of $J(V)$ scans performed $\pm 2.0 \mathrm{~V}$ in order to calculate $R$ after various pulse times, i.e. $2 \mathrm{~min}=$ scans $1-5,5 \mathrm{~min}=$ scans $6-10,10 \mathrm{~min}=$ scans $11-15,15 \mathrm{~min}=$ scans $16-20,30 \mathrm{~min}=$ scans $21-25,45 \mathrm{~min}=$ scans 26-30, $60 \mathrm{~min}=$ scans 31-35 (magenta). (b) Semi-log plot of $R(60 \mathrm{~min})$ vs. Applied Voltage Pulse, showing that $R$ increases exponentially with the applied voltage pulse.

By comparing the value of $R$ obtained when applying a pulse of $+1.0 \mathrm{~V}$ with the increase in the value of $R$ from only accumulative $|J|(V)$ scans $\pm 2.0 \mathrm{~V}$, i.e. without any pulse being applied (as shown in Figure 6.2c), it can be seen that a pulse of $+1.0 \mathrm{~V}$ for an accumulative time of 60 min has only a minimal effect on the value of $R$ obtained (Figure 6.5a). In other words, the 5 scans performed $\pm 2.0 \mathrm{~V}$ to determine the value of $R$, after each pulse period of $+1.0 \mathrm{~V}$ has been applied, have a greater effect on $R$ than the pulse of $+1.0 \mathrm{~V}$ itself. 


\subsubsection{Control Measurements}

Thus far all data presented have been obtained from measurements performed on junction 4.1 which contains a G1-PPI- $(\mathrm{Fc})_{4}$ functionalized dendrimer. In Chapter 4 this junction was found to rectify currents with an $R$ of $7.7\left(\sigma_{\log }=3.1\right)$ for measurements performed $\pm 2.0 \mathrm{~V}$. The rectification observed was attributed to the Fc functional groups of the dendrimer having accessible HOMO levels asymmetrically located within the junction, positioned close and coupled with the $\left(\mathrm{Ga}_{2} \mathrm{O}_{3}\right)$ EGaIn top electrode. To determine if the large increase in rectification observed after applying a voltage pulse can be once again attributed to the $\mathrm{Fc}$ moieties, or may be due to other factors within the junction, various voltage pulses were also applied to control junctions that do not possess accessible HOMO levels, that is junction 4.3 and junction 4.4. Both junction 4.3, which contains a G1-PPI-(Ad) $)_{4}$ dendrimer, and junction 4.4 , which is the bare $\beta C D$ SAM, were found not to rectify current in Chapter 4, giving values of $R$ of $0.7\left(\sigma_{\log }=2.5\right)$ and $1.0\left(\sigma_{\log }=3.0\right)$, respectively, for measurements performed $\pm 2.0 \mathrm{~V}$. However, when applying various voltage pulses to both control junctions, the value of $R$ increased in the same manner as it increased in junction 4.1 (Figure 6.6). Therefore, the increase in $R$, upon applying a pulse, is independent of the supramolecular structure within the tunneling junction, and thus the origin of the increase in $R$, must be due to another component or interface of the supramolecular tunneling junction. For all data concerning the various voltage pulses applied to junctions 4.1, 4.3 and 4.4, see appendix, section A.6.2
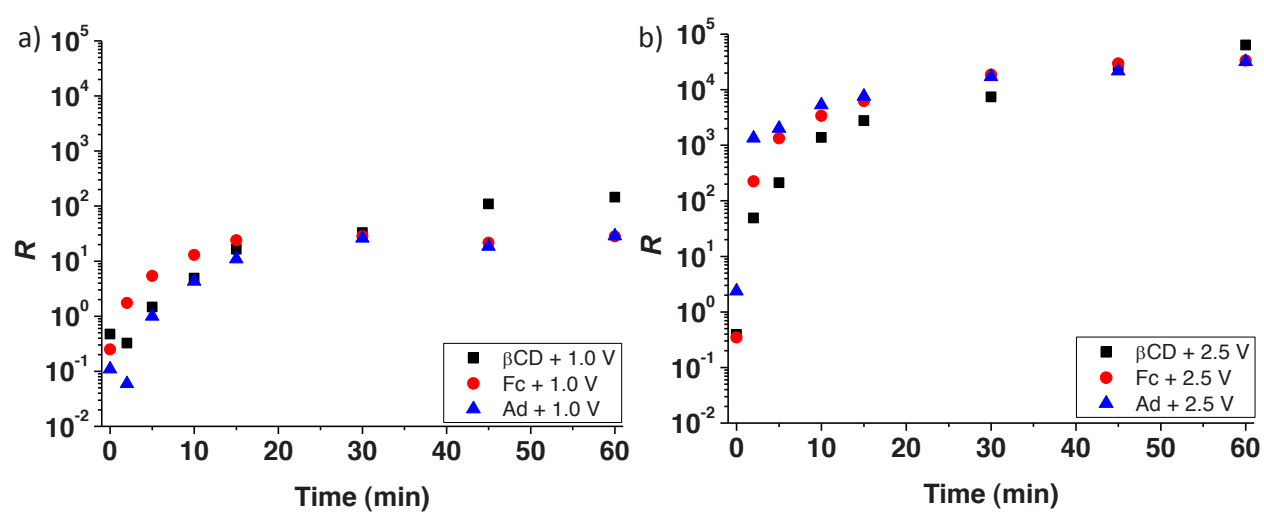

Figure 6.6: Semi-log plots of the increase in rectification for three different supramolecular junctions after the pulse was applied at two different voltages of $+1.0 \mathrm{~V}$ (a) and $+2.5 \mathrm{~V}$ (b) for an accumulative time of $60 \mathrm{~min}$, with $R$ being calculated in the same manner as previously shown in Figure $6.5 \mathrm{a} \mathrm{Au}^{\mathrm{TS}}$. $\beta C D S A M / /\left(\mathrm{Ga}_{2} \mathrm{O}_{3}\right)$ EGaIn (4.4) (black square), $\mathrm{Au}^{\mathrm{TS}}-\beta \mathrm{CDSAM} / \mathrm{G} 1-\mathrm{PPI}-(\mathrm{Fc})_{4} / /\left(\mathrm{Ga}_{2} \mathrm{O}_{3}\right) \mathrm{EGaIn}$ (4.1) (red circle) and $\mathrm{Au}^{\mathrm{TS}}-\beta C D S A M / G 1-P P I-(\mathrm{Ad})_{4} / /\left(\mathrm{Ga}_{2} \mathrm{O}_{3}\right)$ EGaIn (4.3) (blue triangle). 


\subsubsection{The Origin of the Increase in $R$ in EGaIn Supramolecular Tunneling Junctions}

The supramolecular tunneling junction is a complex entity, and thus to investigate the origin of $R$, it is advantageous to visualize the supramolecular tunneling junction as a system of different layers with each of their interfaces. From top to bottom, joining the $\mathrm{Au}^{\mathrm{TS}}$ surface and the heptathioether $\beta C D$ SAM is the Au-S bond, the dendrimer is then adsorbed to the $\beta C D$ SAM via the non-covalent hydrophobic interactions between the terminal functional group of the dendrimer and the inner cavity of the $\beta C D$, the top $\left(\mathrm{Ga}_{2} \mathrm{O}_{3}\right)$ EGaIn is applied with the Van der Waals interaction of the $\mathrm{Ga}_{2} \mathrm{O}_{3}$ layer on the surface of the EGaIn with the terminal function group of the dendrimer (or the $\beta C D$ in the case of junction 4.4), and finally the ill-defined $\mathrm{Ga}_{2} \mathrm{O}_{3}$ layer on the surface of the EGaIn.

XPS was used to investigate the possible rearrangement of the heptathioether $\beta C D$ SAM on the $\mathrm{Au}^{\mathrm{TS}}$ surface, due to the labile $\mathrm{Au}-\mathrm{S}$ bond, and also the possible formation of metal filaments along the molecular components due to the application of the $\left(\mathrm{Ga}_{2} \mathrm{O}_{3}\right)$ EGaIn top electrode to the supramolecular layer. To test this hypothesis, the $\beta C D$ SAM on $\mathrm{Au}^{\mathrm{TS}}$ was examined before and after it had been subjected to a voltage pulse of $+2.5 \mathrm{~V}$ for a period of 15 min. Thus, the advantage of the flexibility of the EGaIn technique was exploited to contact SAMs electrically as this technique makes it possible to assemble a junctionlowering the cone-shaped $\left(\mathrm{Ga}_{2} \mathrm{O}_{3}\right)$ EGaIn top-electrode using the micromanipulator, perform measurements, and disassemble the junction-lifting the cone-shaped $/ /\left(\mathrm{Ga}_{2} \mathrm{O}_{3}\right)$ EGaIn topelectrode using the same micromanipulator, which exposed the SAM that had originally been embedded in the $\mathrm{Au}^{\mathrm{TS}}-\beta \mathrm{CDSAM} / /\left(\mathrm{Ga}_{2} \mathrm{O}_{3}\right) \mathrm{EGaIn}$ (junction 4.4) structure. When comparing the XPS spectra obtained on areas of the sample where the supramolecular layer had been exposed to the voltage pulse, to the XPS spectra obtained on areas where no voltage pulse had been applied, there were no clear differences observed (see appendix section A.6.3). Therefore, there was no re-arrangement of the heptathioether $\beta C D$ SAM on the $\mathrm{Au}^{\mathrm{TS}}$ surface, and no formation of metal filaments along the molecular components due to the application of the $\left(\mathrm{Ga}_{2} \mathrm{O}_{3}\right)$ EGaIn top electrode and the voltage pulse. Thus, these two hypothesized phenomena are not the origin of the increase in $R$. 
To investigate if the PPI dendrimer core is the cause of, or indirectly attributes to the increase in $R$, two additional supramolecular tunneling junctions containing a different dendrimer core were investigated. One junction contained a generation zero adamantyl functionalized poly(amido amine) (PAMAM) dendrimer absorbed to the $\beta C D \mathrm{SAM},\left(\mathrm{Au}^{\mathrm{TS}}\right.$ $\beta C D S A M / G 0-P A M A M-(A d)_{4} / /\left(\mathrm{Ga}_{2} \mathrm{O}_{3}\right)$ EGaIn (5.2)), and the other a generation zero adamantyl functionalized-with additional ethylene glycol linker-PAMAM dendrimer adsorbed to the $\beta C D$ SAM $\left(\mathrm{Au}^{\mathrm{TS}}-\beta \mathrm{CDSAM} / \mathrm{G} 0-\mathrm{PAMAM}-\left((\mathrm{EG})_{3}-(\mathrm{Ad})_{4} / /\left(\mathrm{Ga}_{2} \mathrm{O}_{3}\right)\right.\right.$ EGaIn (5.4)) (Figure 6.1) (Synthesis of the compound G0-PAMAM-((EG) $\left.)_{3}-\mathrm{Ad}\right)_{4}$ is described in Chapter 5). Based on previously published data, adamantyl-functionalized dendrimers were used to ensure that the terminal functional moiety will not be the origin of the rectification. ${ }^{[4]}$ Changing the dendrimer core from PPI to PAMAM and PAMAM with an additional ethylene glycol linker, changes the chemical structure of the molecular assembly within the tunneling junction, and also changes the number of terminal functional moieties that are able to bind to the supramolecular platform, which, as explained in Chapter 5 influences the packing density of the dendrimers on the supramolecular platform. The PAMAM dendrimer with an additional ethylene glycol linker (junction 5.4) makes it possible for all four terminal moieties to bind with the supramolecular platform (with no free terminal moieties to point upwards and form a van der Waals contact with the $\mathrm{Ga}_{2} \mathrm{O}_{3}$ layer on the surface of the EGaIn) and the normal PAMAM core (junction 5.2) allows for three of its four terminal moieties to bind with the supramolecular platform (with one free terminal moiety to form a van der Waals contact with the $\mathrm{Ga}_{2} \mathrm{O}_{3}$ layer). The tetravalent and trivalent interactions with the $\beta C D$ SAM allow both dendrimers to form a densely packed dendrimer layer on the $\beta C D$ SAM, giving a better percentage coverage than the PPI dendrimers used in junctions 4.1 and 4.3 (Table 6.1 on page 160). ${ }^{[7]}$ This minimizes the formation of 'thin area defects' and thus minimizes the $\left(\mathrm{Ga}_{2} \mathrm{O}_{3}\right)$ EGaIn top electrode contacting the supramolecular platform (as discussed in Chapter 5). Therefore, the supramolecular tunneling junctions containing PAMAM dendrimers can also investigate whether the $\left(\mathrm{Ga}_{2} \mathrm{O}_{3}\right)$ EGaIn top electrode contacting the supramolecular platform could also be the cause of the increase in $R$.

Figures $6.7 \mathrm{a}$ and c show $25|J|(V)$ scans $\pm 2.0 \mathrm{~V}$ on junctions 5.2 and 5.4, respectively. The black scans represent 20 scans performed before a voltage pulse was applied, and the red scans (scans 21-25) represent 5 scans performed after a voltage pulse of $+2.5 \mathrm{~V}$ had been applied for $5 \mathrm{~min}$. Figures $6.7 \mathrm{~b}$ and d display the resulting value of $R$ from each of the 25 170 
scans for junctions 5.2 and 5.4, respectively. From referring to Figures $6.7 \mathrm{~b}$ and $\mathrm{d}$ it can be seen that the value of $R$ increases slowly for both junctions during the first 20 scans. After applying the voltage pulse, the value of $R$ measured in the preceding scans (scans 21-25) increased for both junctions by more than one order of magnitude. Therefore, even after changing the molecular structure of the dendrimer core and changing the packing density of dendrimer on the supramolecular platform, an increase in $R$ was still observed when a voltage pulse was applied. Thus, these results indicate that the dendrimer itself and the $\left(\mathrm{Ga}_{2} \mathrm{O}_{3}\right)$ EGaIn top electrode being able to contact the supramolecular platform due to the formation of thin area defects, are not the origin of the increase in $R$.
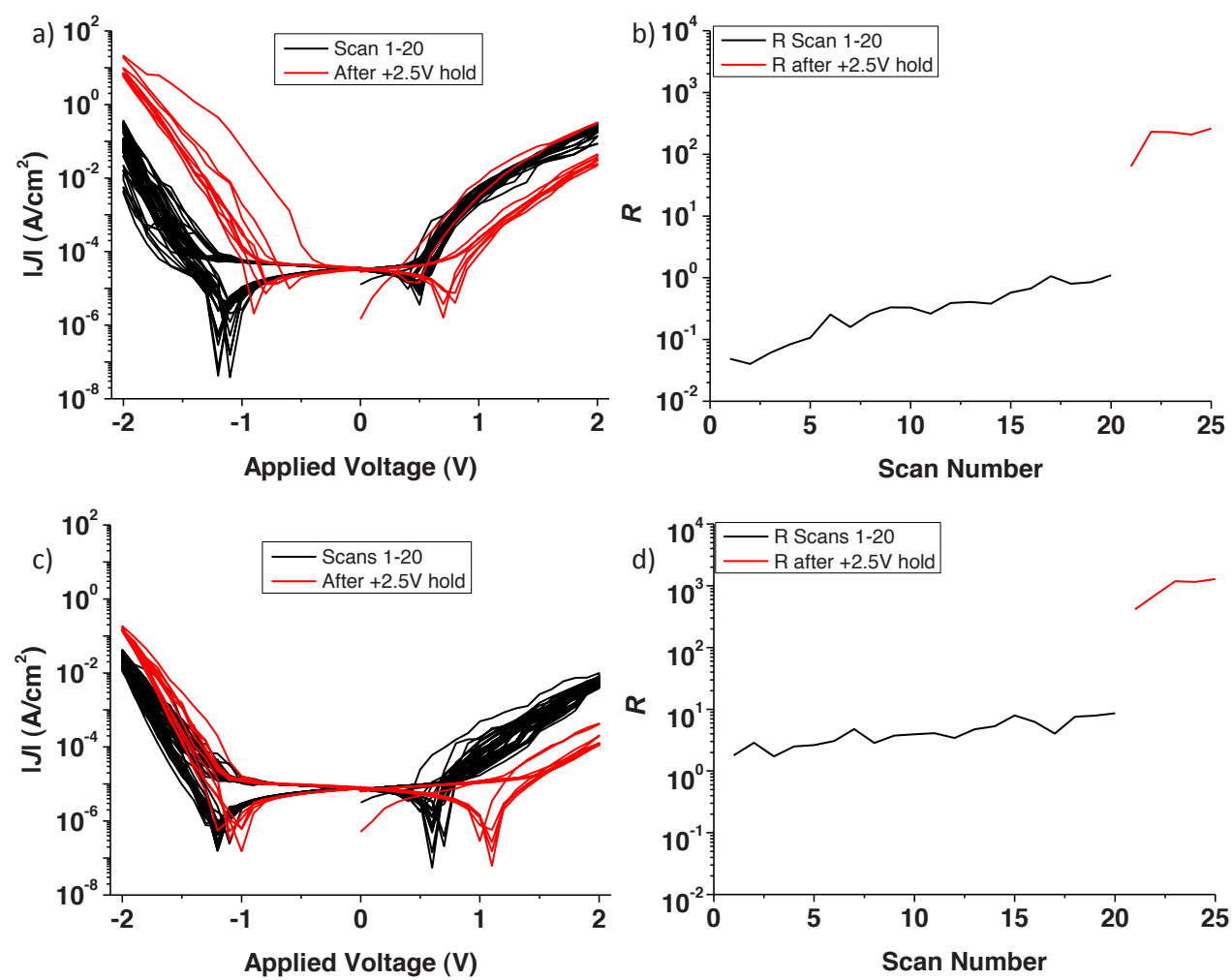

Figure 6.7: Semi-log plots of $|J|(V)$ scans measured $\pm 2.0 \mathrm{~V}$, Scans 1-20 (black), five Scans after applying a voltage pulse of $+2.5 \mathrm{~V}$ (red), (a) $\mathrm{Au}^{\mathrm{TS}}-\beta C D S A M / G 0-P A M A M-(\mathrm{Ad})_{4} / /\left(\mathrm{Ga}_{2} \mathrm{O}_{3}\right)$ EGaIn (junction 5.2), (c) $\mathrm{Au}^{\mathrm{TS}}$ $\beta C D S A M / G 0-P A M A M-E G-(A d)_{4} /\left(\mathrm{Ga}_{2} \mathrm{O}_{3}\right)$ EGaIn (junction 5.4). Semi-log plots of rectification $(R)$ $[\mathrm{R}=|J(-2.0 \mathrm{~V})| /|J(+2.0 \mathrm{~V})|]$ calculated from scans performed in Figure $6.7 \mathrm{a}$ and $\mathrm{c}$ vs. amount of scans performed, (b) $\mathrm{Au}^{\mathrm{TS}}-\beta \mathrm{CDSAM} / \mathrm{G} 0$-PAMAM-(Ad) $4 /\left(\mathrm{Ga}_{2} \mathrm{O}_{3}\right)$ EGaIn (junction 5.2), (d) $\mathrm{Au}^{\mathrm{TS}}-\beta C D S A M / G 0-$ PAMAM-EG-(Ad) $)_{4} /\left(\mathrm{Ga}_{2} \mathrm{O}_{3}\right)$ EGaIn (junction 5.4). 
To exclude charging as the potential cause of the increase in $R$, a voltage pulse of $+2.5 \mathrm{~V}$ was applied across junction 4.1 for a period of $60 \mathrm{~min}$. The $J(V)$ characteristics were investigated directly after the voltage pulse was applied and again after the junction had been left to sit in ambient conditions for an additional 16 hours. After the 16 hour period the value of $R$ did not change significantly and only increased slightly from $5.2 \times 10^{3}$ directly to $1.2 \times 10^{4}$ (Figure 6.8a). A further test of this hypothesis was carried out on a separate junction of 4.1 by applying a voltage pulse at $-2.0 \mathrm{~V}$, after a voltage pulse of $+2.0 \mathrm{~V}$ had been applied to increase the value of $R$ (Figure $6.8 \mathrm{~b}$ and $\mathrm{c}$ ). Interestingly, no change in the subsequent $J(V)$ scan $\pm 2.0 \mathrm{~V}$ was observed. Therefore, charging is unlikely to be the cause of the increase in $R$.
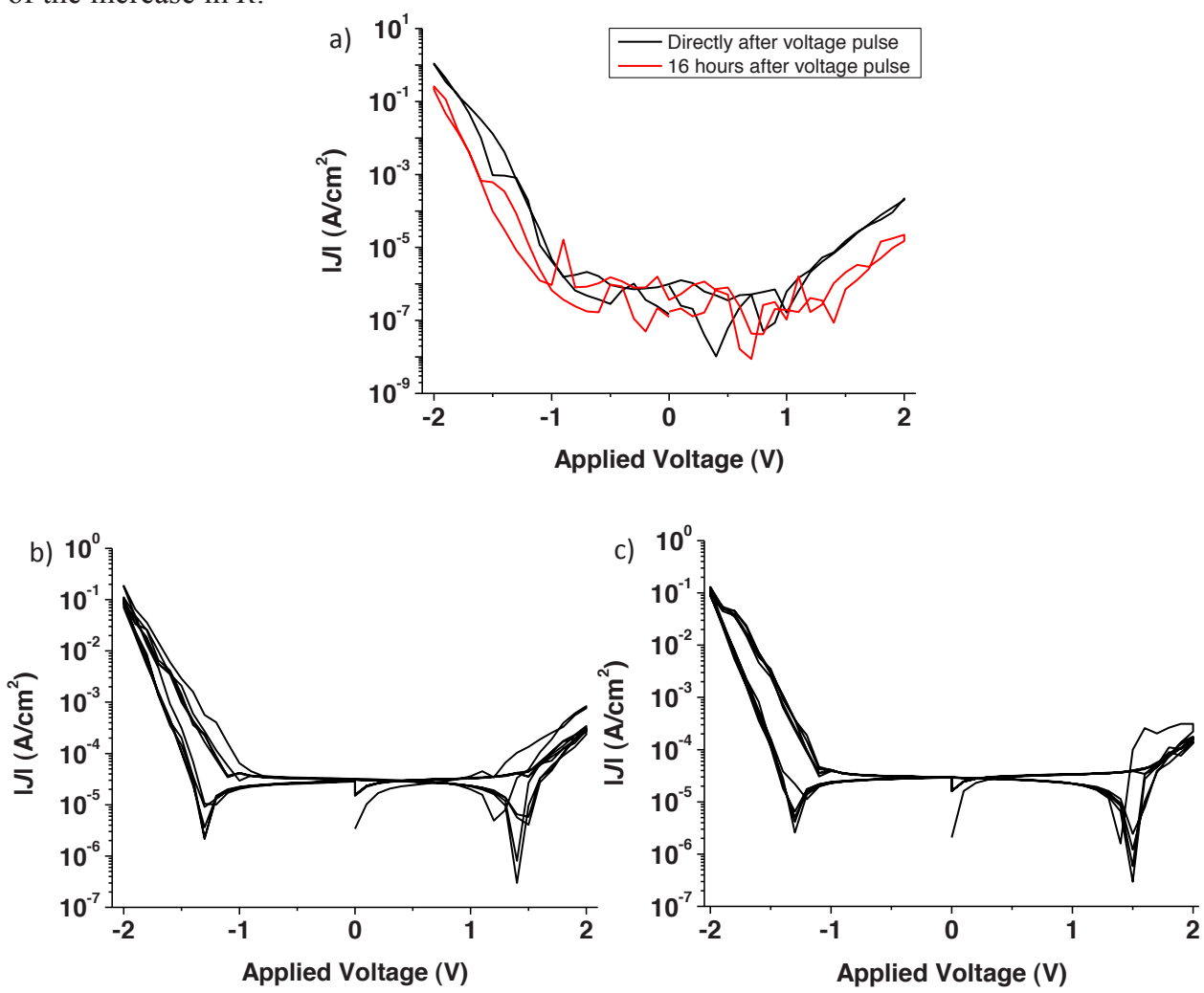

Figure 6.8: $J(V)$ Measurements performed on supramolecular junctions of $\mathrm{Au}^{\mathrm{TS}}-\beta C D S A M / G 1-\mathrm{PPI}$ (Fc) $4 / /\left(\mathrm{Ga}_{2} \mathrm{O}_{3}\right)$ EGaIn (4.1); (a) Semi-log plot of two $|J|(V)$ scans measured $\pm 2.0 \mathrm{~V}$, one measured directly after a voltage pulse of $+2.5 \mathrm{~V}$ had been applied for $60 \mathrm{~min}$ (black scan), the other measured an additional 16 hours later (red scan). (b) Semi-log plot of five $|J|(V)$ scans measured $\pm 2.0 \mathrm{~V}$, after a voltage pulse of $+2.0 \mathrm{~V}$ was applied for $10 \mathrm{~min}$. (c) Semi-log plot of five $|J|(\mathrm{V})$ scans measured $\pm 2.0 \mathrm{~V}$ after an additional voltage pulse of $-2.0 \mathrm{~V}$ was applied to (b) for $20 \mathrm{~min}$. 
The ill-defined $\mathrm{Ga}_{2} \mathrm{O}_{3}$ layer on the surface of the $\left(\mathrm{Ga}_{2} \mathrm{O}_{3}\right)$ EGaIn top electrode was highly suspected as the origin of the increase in $R$. Dickey and co-workers ${ }^{[9]}$ reported anodic growth of the gallium oxide layer on the $\left(\mathrm{Ga}_{2} \mathrm{O}_{3}\right)$ EGaIn alloy and the cathodic dissolution of the gallium layer in slightly acidic electrolyte solutions. To adsorb the dendrimers onto the supramolecular platform, the dendrimers must be dissolved in water by protonating the core amines and complexing the terminal functional moieties $(\mathrm{Ad} / \mathrm{Fc})$ to native $\beta \mathrm{CD}$. Therefore, the supramolecular assemblies formed on the $\mathrm{Au}^{\mathrm{TS}}$ surface contain water and protonated amines with counter ions of mostly $\mathrm{Cl}^{-}$(i.e., similar to an acidic electrolyte solution). Thus, upon applying a voltage of $>+1.0 \mathrm{~V}$ anodic growth of the gallium oxide layer may be occurring within the supramolecular tunneling junctions changing the van der Waals interface into a Schottky barrier, leading to the increase in $R$.

To investigate this phenomenon experiments were performed by Nijhuis and co-workers at the National University of Singapore (NUS) after the completion of the experimental work for this $\mathrm{PhD}$ thesis. Cone-shaped tips of $\left(\mathrm{Ga}_{2} \mathrm{O}_{3}\right) \mathrm{EGaIn}$ were oxidized by applying a voltage pulse of $5.0 \mathrm{~V}$ for $15 \mathrm{~min}$ in an aqueous solution (see experimental). Once dry, the oxidized $\left(\mathrm{Ga}_{2} \mathrm{O}_{3}\right)$ EGaIn tip was placed onto a bare metal surface to create a junction and was found to rectify currents with rectification ratios of 100-1000, with the rectification ratio being determined in only these junctions as $R=|J(-1.5 \mathrm{~V})| /|J(+1.5 \mathrm{~V})|$ (Figure 6.9). The same cone-shaped tips of $\left(\mathrm{Ga}_{2} \mathrm{O}_{3}\right)$ EGaIn before intentional oxidation were found not to form stable contacts with the metal surface and resulted in shorts. Therefore, the anodic growth of the gallium oxide layer on the $\left(\mathrm{Ga}_{2} \mathrm{O}_{3}\right)$ EGaIn alloy is likely to be the origin of the increase in $R$.
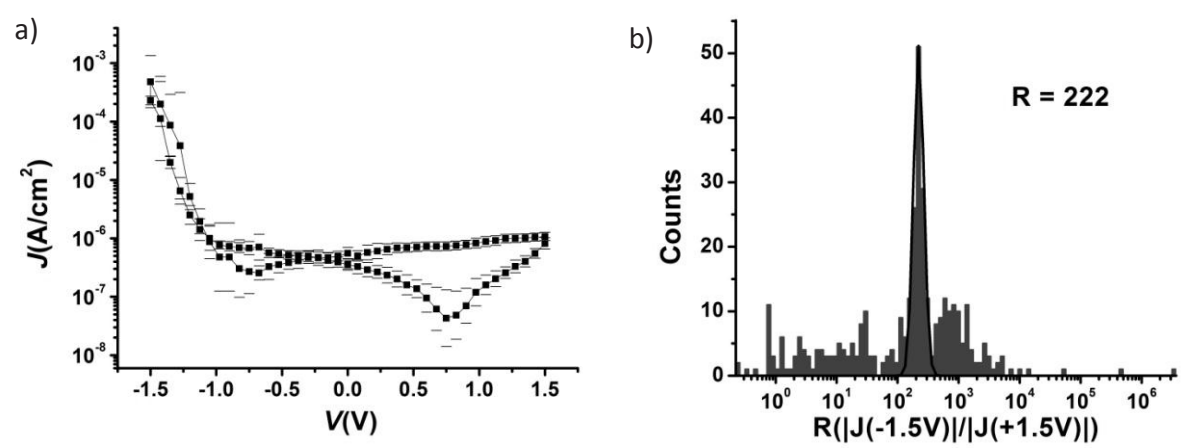

Figure 6.9: (a) Semi-log plot of averaged $|J|$ vs. (V) measurements performed $\pm 1.5 \mathrm{~V}$ on junctions of $\mathrm{Au}^{\mathrm{TS}}-\left(\mathrm{Ga}_{2} \mathrm{O}_{3}\right)$ EGaIn. (b) Histogram of $\log$ rectification ratio $(R)$, where $R=|J(-1.5 \mathrm{~V})| /|J(+1.5 \mathrm{~V})|$. Please note that $R$ is only defined at $\pm 1.5 \mathrm{~V}$ for the junction $\mathrm{Au}^{\mathrm{TS}}-\left(\mathrm{Ga}_{2} \mathrm{O}_{3}\right)$ EGaIn. 


\subsection{Conclusion}

In summary, the $\left(\mathrm{Ga}_{2} \mathrm{O}_{3}\right)$ EGaIn top-electrode technique was used to create supramolecular tunneling junctions that were stable for measuring periods of more than two hours and between constant applied biases of $-2.0 \mathrm{~V}$ and $+2.5 \mathrm{~V}$. When constant biases (voltage pulses) of $>+1.0 \mathrm{~V}$ were applied, the value of $R$ measured for all supramolecular tunneling junctions, regardless of supramolecular and chemical structure, increased. The amount that $R$ increased was dependent on the applied voltage and duration of the pulse, with the largest voltage pulse applied $(+2.5 \mathrm{~V})$, for the longest period of time $(60 \mathrm{~min})$, giving the largest value of $R\left(6.4 \times 10^{4}\right)$. The results obtained when characterizing all components and interfaces of the supramolecular tunneling junctions suggest that the $\mathrm{Ga}_{2} \mathrm{O}_{3}$ layer on the surface of the EGaIn is the origin of the increase in $R$. The $\mathrm{Ga}_{2} \mathrm{O}_{3}$ layer which initially forms a van der Waals contact with the supramolecular layer may undergo anodic growth under an applied positive bias due to the presence of water, and protonated amines with $\mathrm{Cl}^{-}$counter ions. The thick $\mathrm{Ga}_{2} \mathrm{O}_{3}$ layer will then form a Schottky barrier within the junction increasing the value of $R$. The speed of growth and final thickness of the $\mathrm{Ga}_{2} \mathrm{O}_{3}$ layer formed appears to be dependent on the applied bias, with biases of $\sim 1.0 \mathrm{~V}$ showing a minimal increase in $R$, with $R$ then increasing exponentially as a function of the applied bias.

The results presented in this study should make all (potential) EGaIn users wary of the limitations of this technique when performing DC measurements across molecular assemblies that contain water and ions. It is recommended that where possible, to obtain the most informative results, all data should be obtained at a DC bias range no greater than \pm 1.0 $\mathrm{V}$. The influence that applied biases $>+1.0 \mathrm{~V}$ may or may not have on molecular assemblies that do not contain water and ions is currently not known as the molecular assemblies investigated in other studies ${ }^{[3 \mathrm{c}-\mathrm{e}]}$ are not stable for long periods of time at higher biases. The results obtained thus far have only shown that molecular assemblies such as ferrocene alkanethiolates show a minimal change in $R$ over $100|J|(V)$ scans performed $\pm 1.0 \mathrm{~V}$ (E at $1.0 \mathrm{~V} \approx 400 \mathrm{MV} / \mathrm{m})$, with $R=1.0 \times 10^{2}\left(\sigma_{\log }=3.0\right)$ at $\pm 1.0 \mathrm{~V} \cdot{ }^{[3 \mathrm{c}]}$ On the other hand, the ability of the $\left(\mathrm{Ga}_{2} \mathrm{O}_{3}\right)$ EGaIn technique to create junctions with large values of $R$ in a controlled fashion, could prove useful in other device fabrication strategies. 


\subsection{Experimental Details}

Materials: Preparation of heptathioether-functionalized $\beta$-cyclodextrin ${ }^{[6 a]}$ and G1-PPI$(\mathrm{Fc})_{4}{ }^{[10]}$ G1-PPI-(Ad) $)_{4}$, G1-PAMAM-(Ad $)_{4}{ }^{[11]}$ and G0-PAMAM-(EG-Ad $)_{4}{ }^{[12]}$ dendrimers was described previously. All compounds were characterized with Nuclear Magnetic Resonance Spectroscopy $\left({ }^{1} \mathrm{H}\right.$ NMR) and Mass Spectrometry (MALDI ToF and ESI-MS), with all compounds yielding similar results to what has been previously published. Eutectic Gallium Indium was used as purchased from Aldrich.

\section{Method:}

\section{SAM Formation}

Ultra flat substrates of thin films of gold metal on a polymer supported by a glass slide, were obtained by delaminating an evaporated film of gold from a $\mathrm{Si} / \mathrm{SiO}_{2}$ template (the 'mechanical template-stripping' procedure (TS)) as previously described in detail. ${ }^{[13]} \beta C D$ SAMs were prepared by immersing freshly cleaved ultra-flat gold substrates into a $0.1-1 \mathrm{mM}$ solution of $\beta C D$ dissolved in ethanol for $16 \mathrm{hrs}$ at $60^{\circ} \mathrm{C}$. The substrates were removed and placed in a vial of fresh warm ethanol with the vial being gently agitated for one to two minutes. After removal they were further rinsed with ethanol (room temperature) and MilliQ water, and then dried under a stream of dry nitrogen. As previously published, Electrochemical Impedance Spectroscopy was used to characterize control $\beta C D$ SAMs and yielded similar results.

\section{Dendrimer Absorption}

Substrates that were to contain dendrimers were immersed in an aqueous solution of the corresponding dendrimer- $\beta C D$ assembly; G1-PPI-(Fc) $)_{4}$, G1-PPI-(Ad) $)_{4}$, G0-PAMAM-(Ad) and (G0-PAMAM-(EG-Ad) 4 ) dendrimer, $2 \mathrm{mM}$ in $\mathrm{Ad} / \mathrm{Fc}$ concentration in the presence of $2.1 \mathrm{mM}$ of $\beta \mathrm{CD}$ at $\mathrm{pH}=2$ for at least $1 \mathrm{hr}^{[7 \mathrm{a}]}$ Subsequently the samples were rinsed with MilliQ water and dried under a stream of dry nitrogen. As previously published, ${ }^{[7 \mathrm{a}, 7 \mathrm{~b}]}$ Surface Plasmon Resonance and Cyclic Voltammetry were used to characterize the control $\beta C D$ SAM + dendrimer assemblies and yielded similar results.

\section{EGaIn Junction Set-up}

The EGaIn top contact $75.5 \%$ Ga $24.5 \%$ In by weight was formed by bifurcating a drop of EGaIn between a needle and a clean film of $\mathrm{Au} .{ }^{[1 \mathrm{~b}]}$ The resulting conical tip was carefully 
brought into contact with the surface of the molecular structure. A wire directly attached to the metal needle on the syringe connected the EGaIn electrode electrically with an electrometer (Keithley 6430). The supporting Au substrate served as the common (ground) electrode by means of a gold needle that penetrated the SAM and contacted the Au directly. A triaxial cable connected the two electrodes to an external amplifier. The electrometer applied a bias, $V$, across the junction. A positive value of $V$ corresponded with EGaIn being biased positively with respect to the Au. The entire setup, except the source-meter was housed in a home-built aluminum Faraday cage.

\section{Prolonged Scanning Measurements}

Current density $(J)$ was measured as a function of voltage $(V) .100$ scans were measured $\pm 2.0 \mathrm{~V}$, with one scan consisting of a voltage sweep, $0.0 \mathrm{~V} \rightarrow+2.0 \mathrm{~V} \rightarrow 0.0 \mathrm{~V} \rightarrow-2.0 \mathrm{~V} \rightarrow$ $0.0 \mathrm{~V} .|J|$ was measured at each voltage value (step size) of $0.10 \mathrm{~V}$, in both sweep directions, giving a total of 82 points within a single scan. All $J(V)$ scans in this study were performed using this method. $R$ was calculated for each of the individual scans using the equation $R=|J(-2.0 \mathrm{~V})| /|J(+2.0 \mathrm{~V})|$, with all $\mathrm{R}$ values in this study being calculated in the same manner.

\section{High Voltage Measurements}

On a single junction area, in order to calculate the initial value of $R$, one $J(V)$ scan was measured $\pm 2.0 \mathrm{~V}$. A voltage pulse was then applied at $+2.0 \mathrm{~V},-2.0 \mathrm{~V}$ or $+2.5 \mathrm{~V}$ (each different voltage pulse was applied on a fresh junction) for a period of 15 minutes with $|J|$ being measured every 0.1 seconds. An additional five $J(V)$ scans were performed $\pm 2.0 \mathrm{~V}$, with $R$ being calculated for each individual scan, then averaged to give the final $R$ value.

\section{Rectification Measured Vs. Various Voltages over a prolonged period}

On a single junction area, four representative $J(V)$ scans were measured $\pm 2.0 \mathrm{~V}$ to give $R$ at Time $=0$ minutes. A voltage pulse was then applied at $+1.0 \mathrm{~V},+1.5 \mathrm{~V},+2.0 \mathrm{~V}$ or $+2.5 \mathrm{~V}$ for an accumulative time of 60 minutes, with $|J|$ being measured every 0.1 seconds. After 2 minutes, 5 minutes, 10 minutes, 15 minutes, 30 minutes, 45 minutes and 60 minutes, five $J(V)$ scans were performed $\pm 2.0 \mathrm{~V}$, with $R$ being calculated for each individual scan, then averaged to give the final $R$ value. 


\section{Oxidation of EGaIn top-electrode}

Please note that these experiments were performed by Nijhuis and co-workers at the National University of Singapore (NUS) after the completion of the experimental work for this PhD thesis.

A $\left(\mathrm{Ga}_{2} \mathrm{O}_{3}\right)$ EGaIn tip was immersed into a beaker with $50 \mathrm{~mL}$ of water by a micromanipulator. Pt was used as the counter electrode. The EGaIn/water/Pt simple circuit was biased $0.0 \mathrm{~V} \rightarrow+5.0 \mathrm{~V} \rightarrow 0.0 \mathrm{~V}$ using a computer controlled source meter (Keithley). The tip was allowed to dry under ambient conditions overnight after removal from the water. To form the junction $\mathrm{Au}^{\mathrm{TS}}-\left(\mathrm{Ga}_{2} \mathrm{O}_{3}\right)$ EGaIn, the dried oxidized tip was brought into contact with bare $\mathrm{Au}^{\mathrm{TS}}$ using the micromanipulator. $I(V)$ curves were measured by grounding at the bottom $\mathrm{Au}^{\mathrm{TS}}$ electrode and bias at the $\left(\mathrm{Ga}_{2} \mathrm{O}_{3}\right)$ EGaIn top electrode.

\subsection{References}

[1] a)M. A. Rampi, O. J. A. Schueller, G. M. Whitesides, Appl. Phys. Lett. 1998, 72, 1781-1783; b)R. C. Chiechi, E. A. Weiss, M. D. Dickey, G. M. Whitesides, Angew. Chem. Int. Ed. 2008, 47, 142-144; c)H. B. Akkerman, P. W. M. Blom, D. M. de Leeuw, B. de Boer, Nature 2006, 441, 69-72; d)R. L. McCreery, J. Wu, R. P. Kalakodimi, Phys. Chem. Chem. Phys. 2006, 8, 25722590.

[2] M. M. Thuo, W. F. Reus, C. A. Nijhuis, J. R. Barber, C. Kim, M. D. Schulz, G. M. Whitesides, J. Am. Chem. Soc. 2011, 133, 2962-2975.

[3] a)H. V. H. Valkenier, E. H. Huisman, P. A. van Hal, D. M. de Leeuw, R. C. Chiechi, J. C. Hummelen, J. Am. Chem. Soc. 2011, 133, 4930-4939; b)D. Fracasso, H. Valkenier, J. C. Hummelen, G. C. Solomon, R. C. Chiechi, J. Am. Chem. Soc. 2011, 133, 9556-9563; c)C. A. Nijhuis, W. F. Reus, G. M. Whitesides, J. Am. Chem. Soc. 2009, 131, 17814-17827; d)C. A. Nijhuis, W. F. Reus, J. R. Barber, M. D. Dickey, G. M. Whitesides, Nano Lett. 2010, 10, 36113619; e)C. A. Nijhuis, W. F. Reus, G. M. Whitesides, J. Am. Chem. Soc. 2010, 132, 1838618401.

[4] K. S. Wimbush, W. F. Reus, W. G. van der Wiel, D. N. Reinhoudt, G. M. Whitesides, C. A. Nijhuis, A. H. Velders, Angew. Chem. Int. Ed. 2010, 49, 10176-10180.

[5] M. A. Ratner, B. Davis, M. Kemp, V. Mujica, A. Roitberg, S. Yaliraki, in Molecular Electronics: Science and Technology, Vol. 852 (Eds.: A. Aviram, M. Ratner), 1998, pp. 22-37. 
[6] a)M. W. J. Beulen, J. Bugler, B. Lammerink, F. A. J. Geurts, E. M. E. F. Biemond, K. G. C. van Leerdam, F. C. J. M. van Veggel, J. F. J. Engbersen, D. N. Reinhoudt, Langmuir 1998, 14, 6424-6429; b)M. W. J. Beulen, J. Bügler, M. R. de Jong, B. Lammerink, J. Huskens, H. Schönherr, G. J. Vancso, B. A. Boukamp, H. Wieder, A. Offenhäuser, W. Knol, F. C. J. M. van Veggel, D. N. Reinhoudt, Chem. Eur. J. 2000, 6, 1176-1183.

[7] a)C. A. Nijhuis, F. Yu, W. Knoll, J. Huskens, D. N. Reinhoudt, Langmuir 2005, 21, 7866-7876; b)C. A. Nijhuis, J. Huskens, D. N. Reinhoudt, J. Am. Chem. Soc. 2004, 126, 12266-12267; c)J. Huskens, M. A. Deij, D. N. Reinhoudt, Angew. Chem. Int. Ed. 2002, 41, 4467-4471.

[8] The reason for the larger values of $|J|$ in the first of the five $|J|(V)$ scans after holding the voltage at $+2.0 \mathrm{~V}$ and $-2.0 \mathrm{~V}$ is unclear, however we speculate that it is due to charging of the junction that dissipates upon scanning $\pm 2.0 \mathrm{~V}$.

[9] J. H. So, H. J. Koo, M. D. Dickey, O. D. Velev, Adv. Func. Mater. 2012, 22, 625-631.

[10] I. Cuadrado, M. Moran, C. M. Casado, B. Alonso, F. Lobete, B. Garcia, M. Ibisate, J. Losada, Organometallics 1996, 15, 5278-5280.

[11] M. Baars, A. J. Karlsson, V. Sorokin, B. F. W. de Waal, E. W. Meijer, Angew. Chem. Int. Ed. 2000, 39, 4262-4265.

[12] Chapter 5 of this thesis.

[13] a)E. A. Weiss, G. K. Kaufman, J. K. Kriebel, Z. Li, R. Schalek, G. M. Whitesides, Langmuir 2007, 23, 9686-9694; b)D. Q. Gao, F. Scholz, H. G. Nothofer, W. E. Ford, U. Scherf, J. M. Wessels, A. Yasuda, F. von Wrochem, J. Am. Chem. Soc. 2011, 133, 5921-5930. 


\section{Appendix}

\section{Section A.6.1:}

The stability of the three supramolecular tunneling junctions, $\mathrm{Au}^{\mathrm{TS}}-\beta C D S A M / G 1-P P I-$ $(\mathrm{Fc})_{4} / /\left(\mathrm{Ga}_{2} \mathrm{O}_{3}\right)$ EGaIn (4.1), $\quad \mathrm{Au}^{\mathrm{TS}}-\beta \mathrm{CDSAM} / \mathrm{G} 1-\mathrm{PPI}-(\mathrm{Ad})_{4} / /\left(\mathrm{Ga}_{2} \mathrm{O}_{3}\right)$ EGaIn (4.3) $\mathrm{Au}^{\mathrm{TS}}-$ $\beta C D S A M / /\left(\mathrm{Ga}_{2} \mathrm{O}_{3}\right)$ EGaIn (4.4), were tested by scanning for prolonged periods of time $\pm 2.0 \mathrm{~V}$. Figure A6.1 displays the resulting value of rectification $(R)$ defined as $[\mathrm{R}=|J(-2.0 \mathrm{~V})| /|J(+2.0 \mathrm{~V})|]$ from the 100 scans performed on each supramolecular tunneling junction. It can be seen that as the amount of scans increase the value of $R$ also increases for all supramolecular tunneling junctions, regardless of their molecular structure, with junction 4.1 increasing more than junctions 4.3 and 4.4.

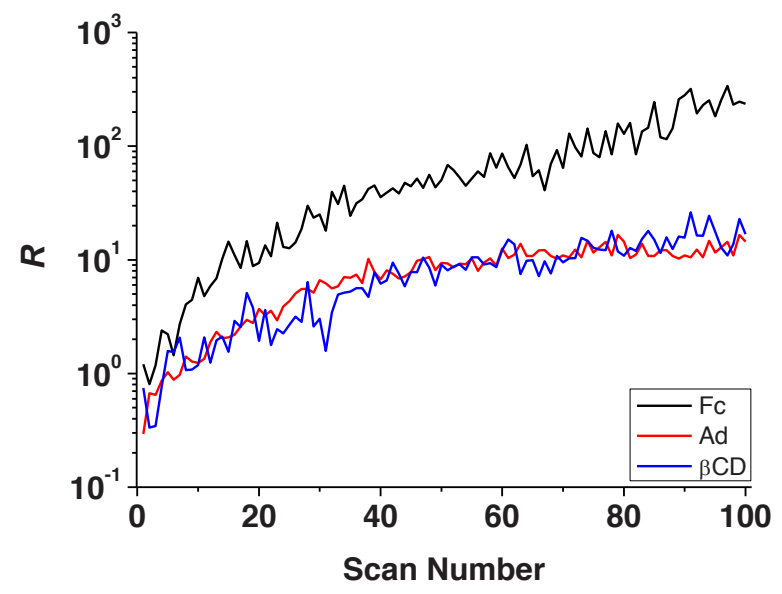

Figure A.6.1: Semi-log plot of $R$ Vs. Scan Number, with, $\mathrm{Au}^{\mathrm{TS}}-\beta \mathrm{CDSAM} / \mathrm{G} 1-\mathrm{PPI}-(\mathrm{Fc})_{4} /\left(\mathrm{Ga}_{2} \mathrm{O}_{3}\right) \mathrm{EGaIn}$ (black, 4.1) $\mathrm{Au}^{\mathrm{TS}}-\beta \mathrm{CDSAM} / \mathrm{G1}$-PPI-(Ad) $)_{4} /\left(\mathrm{Ga}_{2} \mathrm{O}_{3}\right) \mathrm{EGaIn}$ (red, 4.3), and $\mathrm{Au}^{\mathrm{TS}}-\beta \mathrm{CDSAM} / /\left(\mathrm{Ga}_{2} \mathrm{O}_{3}\right) \mathrm{EGaIn}$ (blue, 4.4). 


\section{Section A.6.2:}

To determine the effect that a variety of voltage pulses (different voltage amounts applied over different periods of time), can have on the value of $R$ obtained, voltage pulses of $+1.0 \mathrm{~V},+1.5 \mathrm{~V},+2.0 \mathrm{~V}$ and $+2.5 \mathrm{~V}$ were applied on three different supramolecular tunneling junctions (junctions 4.1, 4.3 and 4.4). After four representative $|J|(V)$ scans $\pm 2.0 \mathrm{~V}$, the voltage pulse was applied for an accumulative time of $60 \mathrm{~min}$, with $R$ being calculated after the pulse had been applied for $2,5,10,15,30,45$ and $60 \mathrm{~min}$, by performing five $|J|(V)$ scans $\pm 2.0 \mathrm{~V}$. The amount of scans chosen to check $R$ is important as accumulative $|J|(V)$ scans $\pm 2.0 \mathrm{~V}$ also increase the value of $R$, vide supra, therefore five scans allowed for an acceptable average value of $R$ to be calculated, whilst minimizing the effect it has on the accumulate value of $R$. Also of high importance is to have a stable junction, as it takes more than two hours complete one full measurement. To show the reproducibility of this trend, the measurements that were carried out across the three different supramolecular junctions (junctions 4.1, 4.3 and 4.4) were performed at least in duplicate (except for the measurements performed on junction 4.4 when holding at $+1.5 \mathrm{~V}$ and $+2.0 \mathrm{~V}$ ) (Figure A6.2e). As shown, all supramolecular junctions displayed a similar increase in $R$ for the voltage pulse applied (Figure A.6.2). 

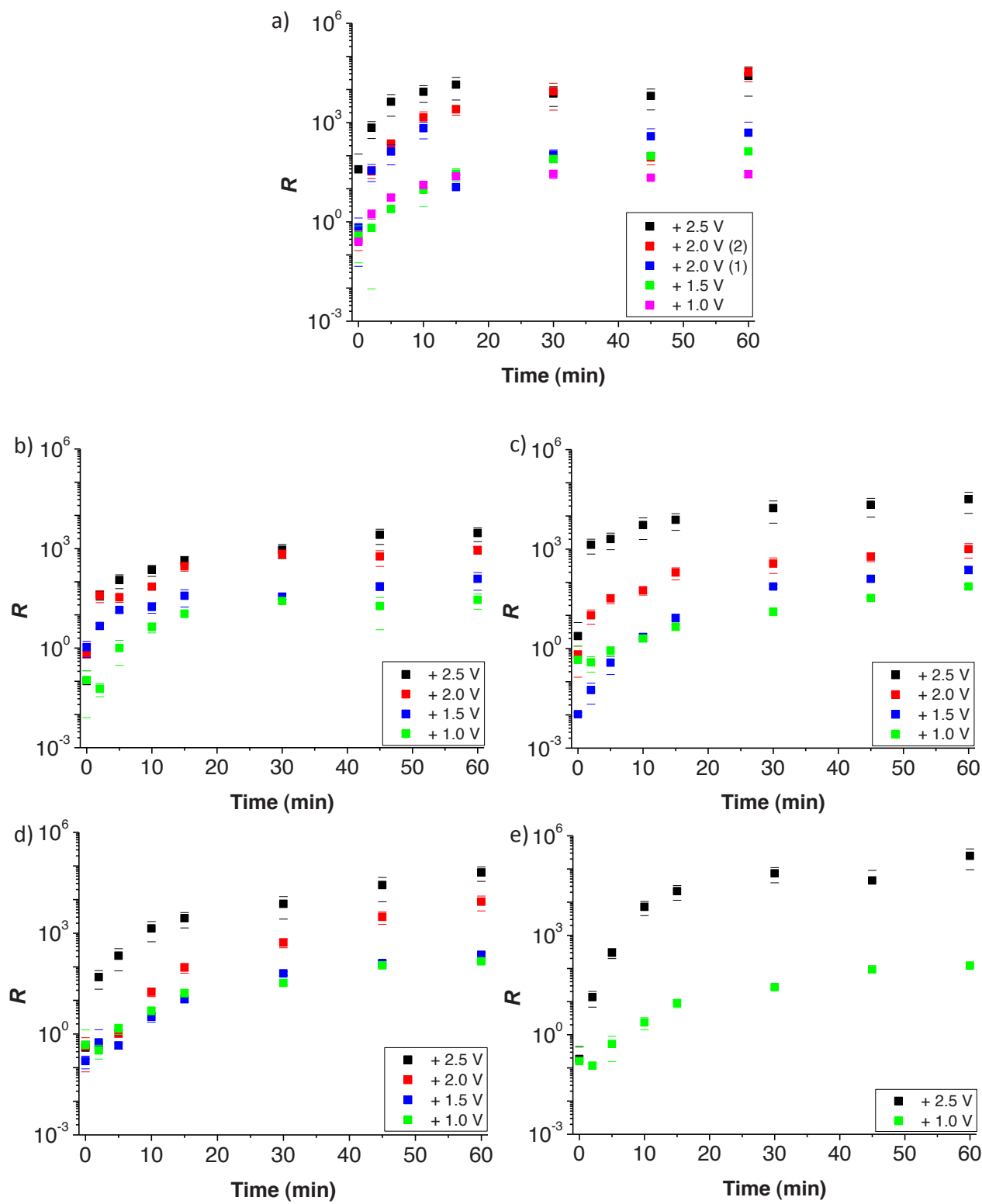

Figure A.6.2: Semi-log plot of the increase in rectification $(R)[\mathrm{R}=|J(-2.0 \mathrm{~V})| / J(+2.0 \mathrm{~V}) \mid]$ in the supramolecular tunneling junctions $4.1,4.3$ and 4.4 , when applying a constant voltage of $+1.0 \mathrm{~V}$ (green), $+1.5 \mathrm{~V}$ (blue), $+2.0 \mathrm{~V}$ (red), $+2.5 \mathrm{~V}$ (black) over an accumulative time of $60 \mathrm{mins}$. After four representative $J(V)$ scans \pm $2.0 \mathrm{~V}$ to determine $R$ at time $(t)=0 \mathrm{~min}, R$ is calculated by performing five $J(V)$ scans $\pm 2.0 \mathrm{~V}$ after the accumulative pulse time of 2, 5, 10, 15, 30, 45 and 60 min.; (a) junction 4.1, (b,c) junction 4.3, (d.e) junction 4.4 . 


\section{Section A.6.3: XPS Data}

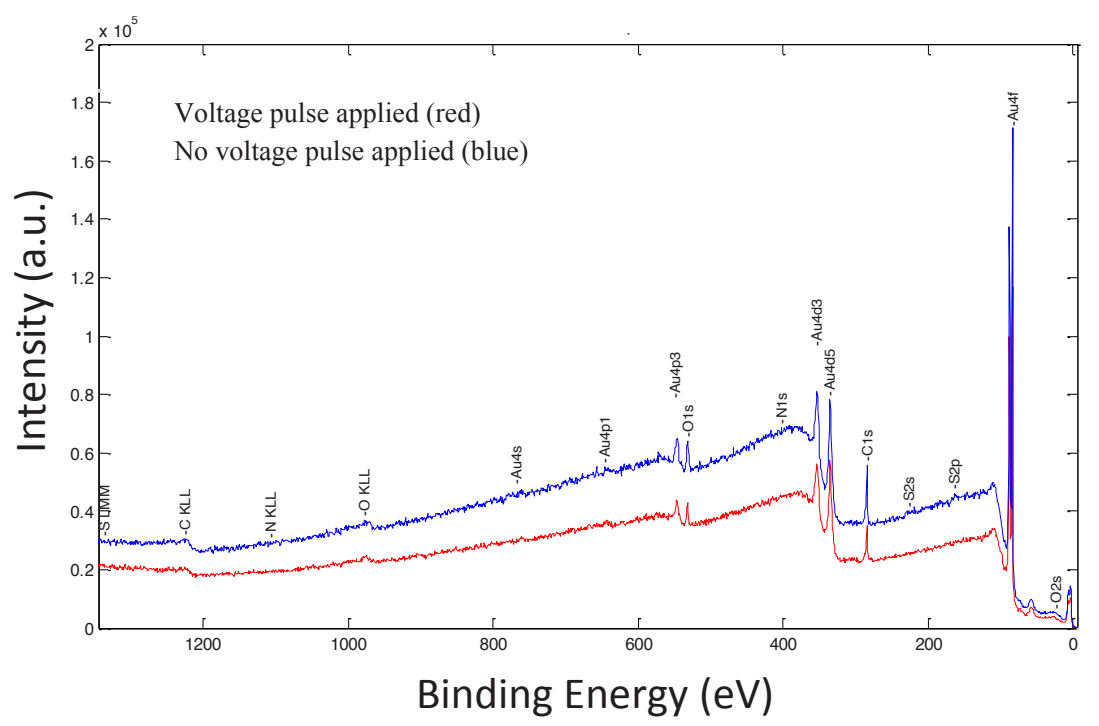

Figure A.6.3: XPS measurements performed on the $\mathrm{Au}^{\mathrm{TS}}-\beta C D S A M$ supramolecular system; in areas where the $\left(\mathrm{Ga}_{2} \mathrm{O}_{3}\right)$ EGaIn top contact has been applied and subjected to a voltage pulse of $+2.5 \mathrm{~V}$, with the $\left(\mathrm{Ga}_{2} \mathrm{O}_{3}\right) \mathrm{EGaIn}$ top then being retracted after the measurement (red spectra), an area where no $\left(\mathrm{Ga}_{2} \mathrm{O}_{3}\right)$ EGaIn top contact was applied and no voltage pulse was applied (blue spectra).

Table A6.1: Relative atomic concentration table

\begin{tabular}{lll}
\hline Atom & $\begin{array}{l}\text { Area where voltage } \\
\text { pulse was applied }\end{array}$ & $\begin{array}{l}\text { Area where no voltage } \\
\text { pulse was applied }\end{array}$ \\
\hline $\mathrm{C}$ & 58 & 63 \\
\hline $\mathrm{S}$ & 4 & 1 \\
\hline $\mathrm{Au}$ & 23 & 21 \\
\hline
\end{tabular}




\section{Electron Induced Dynamics of}

\section{Heptathioether $\beta$-cyclodextrin}

\section{Molecules}

In this Chapter variable temperature scanning tunneling microscopy (STM) and spectroscopy (STS) measurements are performed on hepthathioether $\beta$-cyclodextrin $(\beta C D)$ self-assembled monolayers (SAM) on Au. The $\beta C D$ molecules exhibit very rich dynamical behavior, which is not apparent in ensemble-averaged studies. The dynamics is reflected in the tunneling current-time traces, which were recorded with the STM feedback loop disabled. The dynamics is temperature independent, but increases with increasing tunneling current and sample bias, indicating that the conformational changes of the $\beta \mathrm{CD}$ molecules are induced by electrons that tunnel inelastically. Even for sample biases as low as $10 \mathrm{mV}$ well-defined levels are observed in the tunneling current-time traces. These jumps are attributed to the excitations of the molecular vibration of the macrocyclic $\beta C D$ molecule. These results are of great importance for a proper understanding of charge transport measurements in SAMs.

Part of this chapter was published as A.Kumar, R. Heimbuch, K. S. Wimbush, H. Ateşçi, A. Acun, D. N. Reinhoudt, A. H. Velders, H.J.W. Zandvliet, Small, 2012, 8, 317-322 and is adapted with permission. Copyright (C) 2011 John Wiley and Sons 


\subsection{Introduction}

For the field of molecular electronics to meet its high expectations, ${ }^{[1]}$ there must be a clear fundamental understanding of charge transport through the components in the molecular system. Even though a large variety of approaches have been used to create molecular junctions, ${ }^{[1 \mathrm{c}, 1 \mathrm{~d}]}$ there are currently only a few examples of unambiguous charge transport studies in literature. ${ }^{[2]}$ A majority of the large area devices created and investigated are based on Self Assembled Monolayers (SAMs) immobilised between two electrodes. ${ }^{[2 \mathrm{~d}, 3]}$ However, due to the macroscopic nature of the metal-molecules contacts, the current versus voltage $(I(V))$ data obtained will always present an average of the charge transport events that occur across the array of contacted molecules. Therefore, a detailed understanding of charge transport across single molecules cannot be achieved when investigating the phenomena that occur in molecular assemblies. ${ }^{[4]}$ There have also been limited efforts to interpret the stochastic events, ${ }^{[4 \mathrm{f}, 4 \mathrm{~g}]}$ but a clear understanding of transport through individual entities is still difficult to infer from such large ensembles.

In Chapters 4 and 5 the $I(V)$ characteristics of Supramolecular Tunneling Junctions were described; dendrimers of various terminal functionality, multivalently absorbed on a supramolecular platform of hepthathioether $\beta$-cyclodextrin $(\beta C D)$, immobilized on template stripped $\mathrm{Au}\left(\mathrm{Au}^{\mathrm{TS}}\right)$, with the top contact being applied via the $\left(\mathrm{Ga}_{2} \mathrm{O}_{3}\right)$ EGaIn technique. ${ }^{[5]}$ These supramolecular systems are important as they can act as host molecules for a variety of organic/inorganic molecules for sensing applications and molecular electronics applications. ${ }^{[5-6]}$ These supramolecular systems have been previously characterised with atomic force microscopy $(\mathrm{AFM}),{ }^{[6-7]}$ electrochemistry, cyclic voltammetry $(\mathrm{CV}),{ }^{[6,}{ }^{8]}$ electrochemical impedance spectroscopy (EIS), $\left.{ }^{[6,}{ }^{8 c}\right]$ differential pulse voltammetry $(\mathrm{DPV}),{ }^{[8 \mathrm{~b}-\mathrm{d}]}$ scanning electrochemical microscopy $(\mathrm{SEM}),{ }^{[9]}$ surface plasmon resonance $(\mathrm{SPR})^{[7 \mathrm{a}, 8 \mathrm{~b}, 8 \mathrm{~d}]}$ and SPR and CV combined. ${ }^{[8 \mathrm{~b}, 8 \mathrm{~d}]}$ However, the true mechanism of charge transport remains unclear. As in many of the other tunneling junctions investigated our metal-molecule contacts are of the macroscopic level, and therefore in an attempt to further understand the mechanism of charge transport throughout our assemblies these phenomena were investigated at the level of single molecules, i.e with a scanning tunneling microscope (STM) (Figure 7.1). 
STM is commonly used to study the current voltage characteristics of molecular selfassemblies. However, due to the limited temporal resolution of STM (a few seconds), dynamic events are often averaged out. The temporal resolution of a standard STM can be improved by 6-7 orders of magnitude by recording tunnel current-time traces (time-resolved measurements) at a pre-selected position, while the feedback loop is switched off (Figure 7.1c). Here, the bandwidth of the current-voltage converter $(\sim 100 \mathrm{kHz})$ limits the temporal resolution to $10-20 \mu \mathrm{s}$. This should be compared to a time resolution of only $\sim 1 \mathrm{~ms}$ for position-time experiments where the feedback loop is enabled. 


\subsection{The Scanning Tunneling Microscope (STM) Setup}

a)
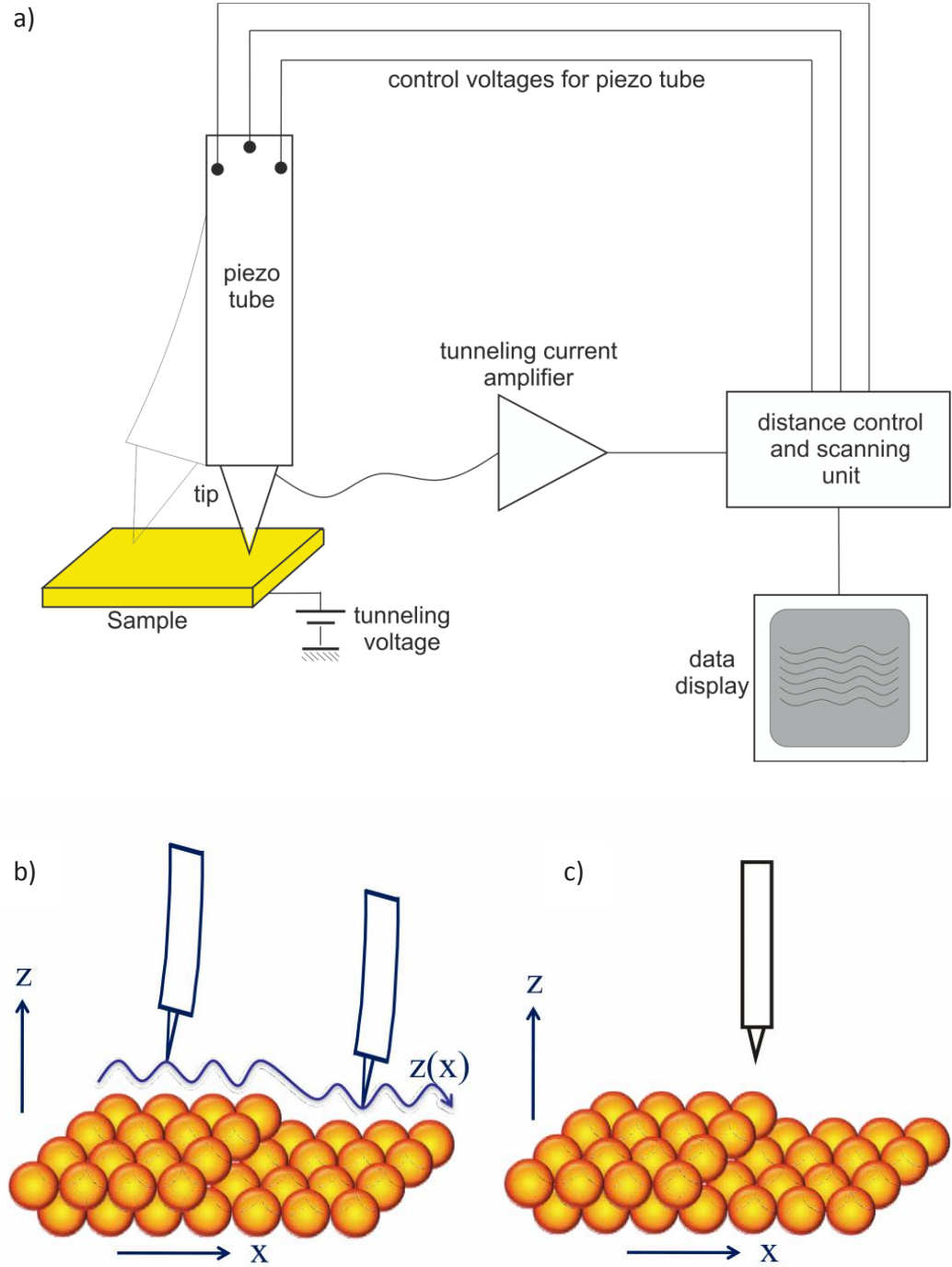

Figure 7.1: (a) Schematic of the Scanning Tunneling Microscope (STM) setup. An atomically sharp tip is mounted on a piezo tube scanner, which makes tiny movements using applied electric fields. (b) During the standard imaging process (commonly referred to as constant-current mode), the tip scans over the surface in the $\mathrm{x}$ and $\mathrm{y}$ direction whilst an electronic feedback loop keeps the tunneling current constant by varying the tip substrate distance, z. (c) Upon performing current-voltage $I(V)$ scans and current-time $I(t)$ measurements the STM tip is placed at a pre-selected position and the feedback loop is switched off. This allows the STM tip to be at a single fixed position during the $I(V)$ and $I(t)$ measurements (Figures (b) and (c) reprinted with permission from. ${ }^{[10]}$ Copyright (C) 2010, American Physical Society). 
The conductance switching and motion of a variety of molecules has been studied using the time resolved technique with the feedback loop disabled. These include single oligo (phenylene ethynylene) molecules embedded in a SAM matrix of dodecanethiolate, ${ }^{[4 \mathrm{~h}]}$ molecular rotors of butyl sulphide, ${ }^{[11]}(\mathrm{t}-\mathrm{Bu})_{4}-\mathrm{ZnPc},{ }^{[12]}$ flipping dimers ${ }^{[13]}$ and flickering of single diarylethene molecules. ${ }^{[14]}$ One recent study carried out by Zandvliet and coworkers $^{[15]}$ displayed stochastic events of a single octanethiol molecule. An STM tip with a single octanethiol molecule attached was moved toward and away from a $\mathrm{Pt} / \mathrm{Ge}(001)$ substrate with a step size of $0.05 \mathrm{~nm}$. At each step, with the feedback loop disabled, $I(V)$ measurements were performed (ten times) by ramping a voltage from $+1.5 \mathrm{~V}$ to $-1.5 \mathrm{~V}$ (Figure 7.2). The authors found that when the STM tip was sufficiently close to the surface (i.e. $0.7-0.8 \mathrm{~nm}$ ), at $+1.5 \mathrm{~V}$ the octanethiol molecule would suddenly jump into contact with the surface, (indicated in the middle of Figure 7.2 by the increase in current) and would then detach from the surface upon approaching a negative sample bias (indicated in the middle of Figure 7.2 by the decrease in current). The threshold voltage for this attachment/detachment phenomenon was found to vary with the distance between the STM tip and $\mathrm{Pt} / \mathrm{Ge}(001)$ substrate, and thus this phenomenon was found to be dependent on the applied electric field, with the threshold electric field being $\sim 4-6 \times 10^{9} \mathrm{~V} \mathrm{~m}^{-1}$. However, all these studies target a single molecule on a surface, ${ }^{[10,16]}$ and thus, as of yet, there have been no time-resolved STM studies carried out on molecular self-assemblies. 


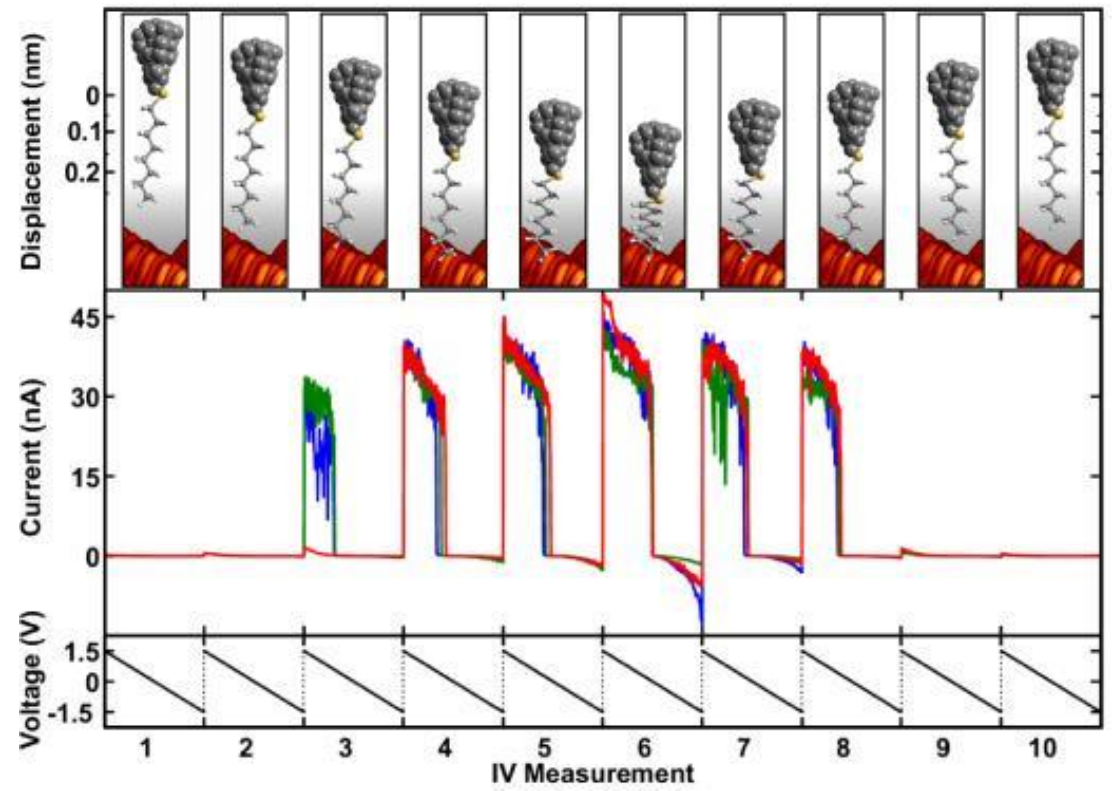

Figure 7.2: Three sets of $I(V)$ curves (each individual curve defined in the middle section as red, blue and green) recorded in series, at various distances $(\mathrm{nm})$ between the STM tip and $\mathrm{Pt} / \mathrm{Ge}(001)$ substrate, with the feedback loop disabled. Top section displays a schematic of the STM tip with the octanethiol molecule attached, at varying tip-substrate distances $(\mathrm{nm})$. Bottom section displays a series of voltage ramps from +1.5 $\mathrm{V}$ to $-1.5 \mathrm{~V}$, as the tip is moved in step sizes of $0.05 \mathrm{~nm}$. Traces $1-6$ correspond to the tips displacement (nm) from the setpoint height (i.e. $0.2 \mathrm{nA}$ and $1.5 \mathrm{~V}$ ) to $0.25 \mathrm{~nm}$ (i.e. tip moving toward the surface), while traces 7 10 correspond to displacement from 0.20 to $0.05 \mathrm{~nm}$ (i.e. tip moving away from surface). After the STM tip has approached the substrate (been displaced) by 0.1 to $0.15 \mathrm{~nm}$ (traces $3-4$ ) the octanethiol molecule suddenly jumps into contact with the surface and after trace 8 , the molecule is out of contact with the surface (Figure reprinted with permission from. ${ }^{[15]}$ Copyright $(C)$ 2012, IOPscience).

In this study time-resolved STM measurements are used to investigate the molecular dynamics and address the electron coupling and vibronic modes of the supramolecular platform used throughout this thesis, $\beta C D$ molecules immobilized on Au surfaces, to obtain a deeper understanding about the charge transport within the molecular system. 


\subsection{Results and Discussion}

Presented and discussed throughout this section will be the STM imaging of the bare flame annealed $\mathrm{Au}$ surface, the $\beta \mathrm{CD}$ SAM on the $\mathrm{Au}$ surface, and the $I(V)$ measurements performed on the $\beta C D$ SAM. This will be followed by the time resolved STM measurements performed on the $\beta C D$ SAM which display molecular conformational changes occurring at both room temperature and $77 \mathrm{~K}$. The amount of these conformational changes increases with increasing tunneling current. Finally, our hypothesis for the observed dynamics of the $\beta C D$ molecules will be presented.

\subsubsection{STM Imaging and $I(V)$ Measurements}

Figure 7.3a shows the molecular structure of a $\beta C D$ molecule ${ }^{[6,17]}$ and a cartoon depicting the arrangement of $\beta C D$ molecules in a SAM on a Au surface with an STM tip positioned at the top. To ensure that the SAMs were formed on well annealed $\mathrm{Au}$ surfaces, the $\mathrm{Au}$ substrates were introduced into the UHV system and imaged. Figure 7.3b is an STM image of the bare Au substrate. The pattern on the terraces is due to the presence of a herringbone reconstruction of the $\mathrm{Au}(111)$ surface, ${ }^{[18]}$ thus indicating very well annealed $\mathrm{Au}$ surfaces. The ordering of the herringbone reconstruction is slightly more irregular than that of an UHV cleaned Au (111), but the presence of this on a flame annealed Au surface is still exceptional. 


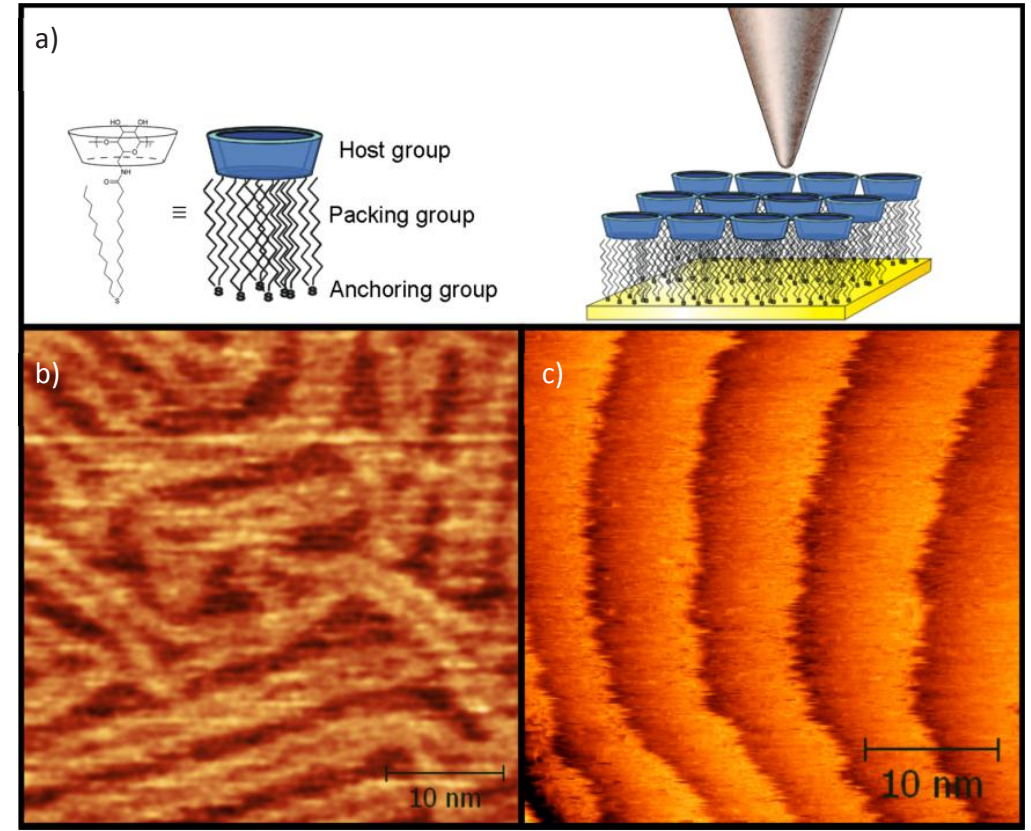

Figure 7.3: (a) Chemical structure of the $\beta C D$ molecule and a cartoon depicting the arrangement of the $\beta C D$ molecules on a Au surface including a STM tip. (b) STM image of a bare Au(111) surface after flame annealing. (c) STM image of a $\beta C D$ SAM on $\operatorname{Au}(111)$.

Figure 7.3c shows an STM image of the $\beta C D$ SAM on a flame annealed Au substrate recorded at room temperature under UHV conditions. The measured step height of $0.25 \mathrm{~nm}$ corresponds to the height of a single layer step of $\mathrm{Au}(111)$. Molecular resolution on the terraces was not achieved. This was not caused by a thermally induced motion of the $\beta C D$ molecules, as imaging at $77 \mathrm{~K}$ also did not produce molecular resolution. This inability to obtain molecular resolution can be due to intermolecular transport parallel to the substrate. Even though molecular resolution is not obtained the $I(V)$ measurements recorded on the $\beta C D$ SAM are substantially different from the $I(V)$ traces recorded on the bare Au substrate. Therefore, it is concluded that the $\beta C D$ SAM is indeed present. Such $I(V)$ traces recorded at the same location are shown in the Figure 7.4. Both $I(V)$ traces show exponential behavior with different exponent decays. At higher bias values, $I(V)$ trace 1 jumps to lower current values, which overlaps with $I(V)$ trace 2 . After the jump to a lower current value, it again jumps back to the original value at a positive bias. That means that the molecular assembly shows a conductance change during the acquisition of the $I(V)$ traces. This may be related to 
the molecular dynamics occurring in the SAM. In order to study the nature of such dynamics time-resolved STM measurements were performed, as discussed below.

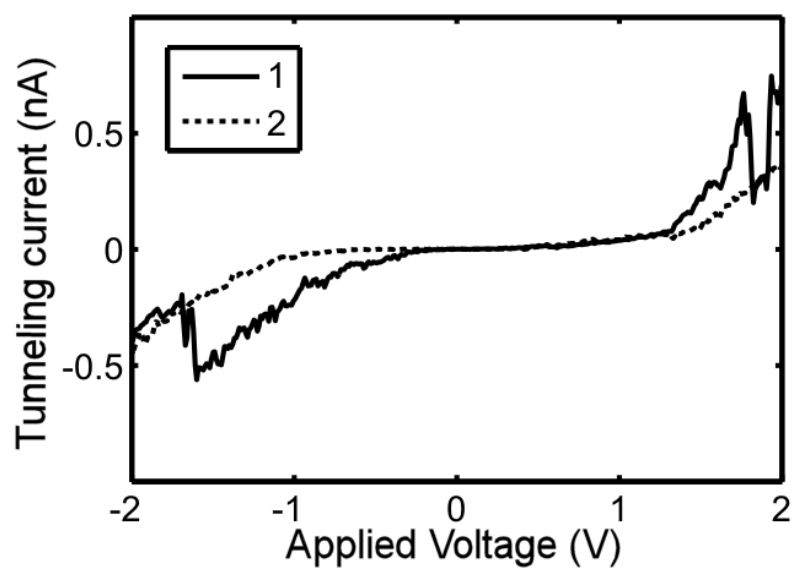

Figure 7.4. $I(V)$ curves of the molecular tunnel junction recorded at room temperature. The set points are $2 \mathrm{nA}$ and $-3 \mathrm{~V}$, respectively. $I(V)$ trace 2 does not exhibit any dynamic changes, whereas $I(V)$ trace 1 jumps back and forth between a low current value and a high current value. The low current value overlaps with $I(V)$ trace 2 .

Interestingly, in $\sim 20 \%$ of the $I(V)$ traces, resonance peaks (appearing as a 'distinct noise') were seen in the measured tunneling current at biases larger than the work function (i.e. here $1.5 \mathrm{eV})$. These resonance peaks occur as the STM is being operated in the field emission and Fowler-Nordheim regimes, with the applied bias voltage being greater than the work function of the tip and substrate. This causes electron standing waves $(n)$ to be present in a triangular potential well in the tip - sample (vacuum) gap. When the applied bias voltage $(V)$ matches the energy level of the electron standing wave (which occurs in a triangular potential well with the relationship $V \alpha n^{2 / 3}$ ) a resonance peak occurs in the measured tunnelling current. This phenomenon is known as Gundlach oscillations. ${ }^{[19]}$ Figure 7.5 displays a plot of $V$ vs. $n^{2 / 3}$ for six resonance peaks that occurred during an $I(V)$ scan on the $\beta C D$ SAM. The applied bias voltage at which each resonance peak occurred closely follows the relationship $V \alpha n^{2 / 3}$, which is true for a triangular potential well. 


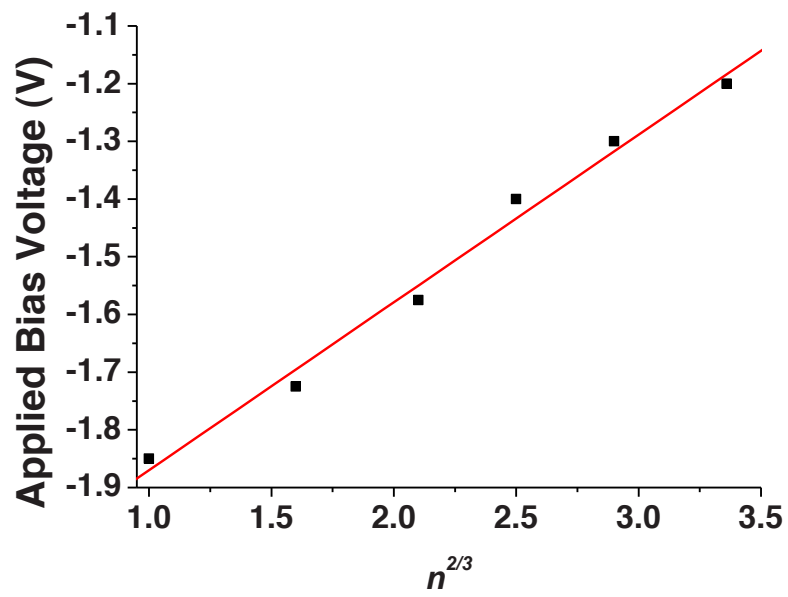

Figure 7.5: Plot of $V$ vs. $n^{2 / 3}$ for six resonance peaks that occurred during $I(V)$ measurements on the $\beta C D$ SAM. The relationship of $V \alpha n^{2 / 3}$ is true for a triangular potential well indicating that the resonance peaks are due to Gundlach oscillations.

\subsubsection{Time-Resolved STM Measurements}

Figure 7.6 shows two time-resolved STM measurements recorded at room temperature, only at areas where flat terraces are present. In Figure 7.6a the $\beta C D$ molecule switches back and forth between a low current level of $\sim 0.3 \mathrm{nA}$ and a high current level of $\sim 0.6 \mathrm{nA}$. These two levels are believed to correspond to two different conformational configurations of the molecule. There are several possible causes for such dynamics observed in the time-resolved traces. For example, the X-ray Photoelectron Spectroscopy (XPS) studies ${ }^{[17]}$ carried out on $\beta C D$ SAM may correlate to different conformational configurations. The XPS studies showed that on average only 3.2 out of the 7 sulfur (S) moieties (anchoring groups) are bound to $\mathrm{Au}$ at any one time. Therefore, during the period of time that the STM measurement is performed, the number of $\mathrm{S}$ moieties of the $\beta \mathrm{CD}$ molecule bound to the $\mathrm{Au}$ surface could change, for example from $3 \mathrm{~S}$-Au interactions to $4 \mathrm{~S}-\mathrm{Au}$ interactions, due to the labile bonding between the sulfur and the $\mathrm{Au}^{\left[{ }^{[20]}\right.}$ Also the sulfur moieties can have 
different adsorption geometries on the $\mathrm{Au}$ surface which is directly related to the conductance through the anchoring groups of the molecules. Therefore, the attachment and de-attachment of the anchoring groups with different adsorption geometries will result in many levels.
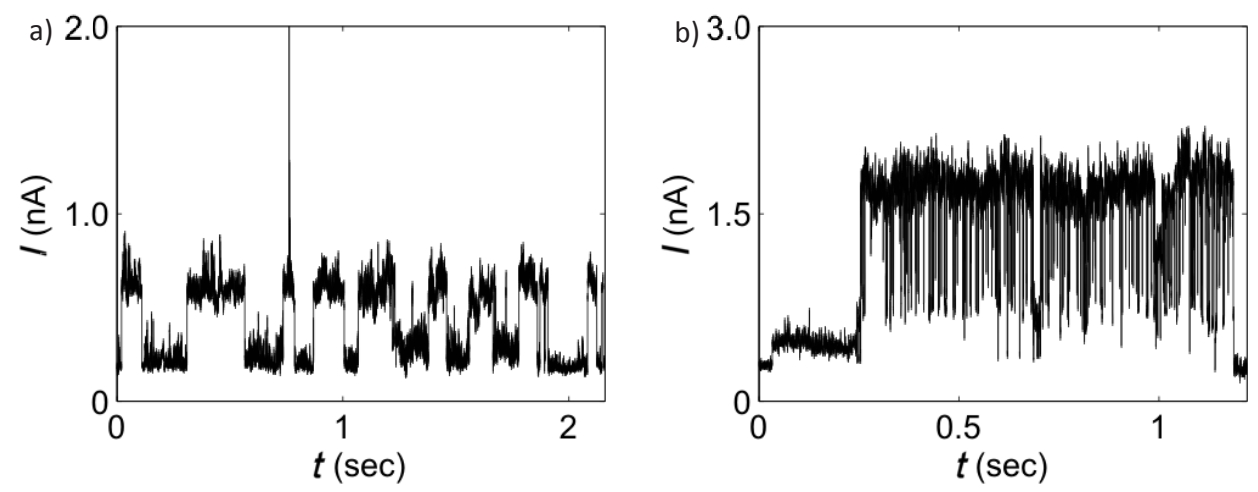

Figure 7.6: Current-time $(I(t))$ traces performed on a $\beta C D$ self-assembled monolayer at $300 \mathrm{~K}$ (a \& b). The sample bias was $-1.25 \mathrm{~V}$.

Intermolecular interactions could also be a reason for the observed switching. As mentioned above these interactions seem to be important as they may be the reason why molecular resolution is not achieved on the surface. The intermolecular interactions can be due to the van der Waals force, or polarization effects which can also lead to the conformational changes in the molecules. One example of multi-level conductance switching is shown in Figure 7.6b. The residence times in the two high current levels are much shorter than the residence times of the low current levels. An analysis of the distribution of residence times of the two high current levels reveals that the switching process between the two levels is fully stochastic, i.e. there is no memory effect. The average residence times at these two levels are $\sim 3.6 \mathrm{~ms}$ and $\sim 0.57 \mathrm{~ms}$, respectively. This difference is most likely due to an energetic asymmetry between the two higher conductance states, as will be discussed below and in Figure 7.9 (page 196).

Figure 7.7 shows two $I-t$ traces recorded at $77 \mathrm{~K}$. The observed dynamics are essentially the same as seen at room temperature and therefore the switching behavior of the $\beta C D$ is not thermally induced. In Figure 7.7a, an $I-t$ trace is shown that exhibits at least seven different current levels. The residence times in all levels are larger than $\sim 100 \mathrm{~ms}$. Figure $7.7 \mathrm{~b}$ shows 
another $I-t$ trace where seven current levels are observed. The residence times are however much shorter as compared to the trace in Figure 7.7a. Although Figures 7.7a and 7.7b both exhibit at least seven different levels (small red lines), the relative ratios of the different current levels and average residence times reveal that there are different conformational configurations of the $\beta C D$ molecule present. However, it is not possible to be sure about the exact number of molecules involved in the tunneling process, due to the possibility of lateral transport, so it is also possible that the slow and fast switching processes are related to different molecules.
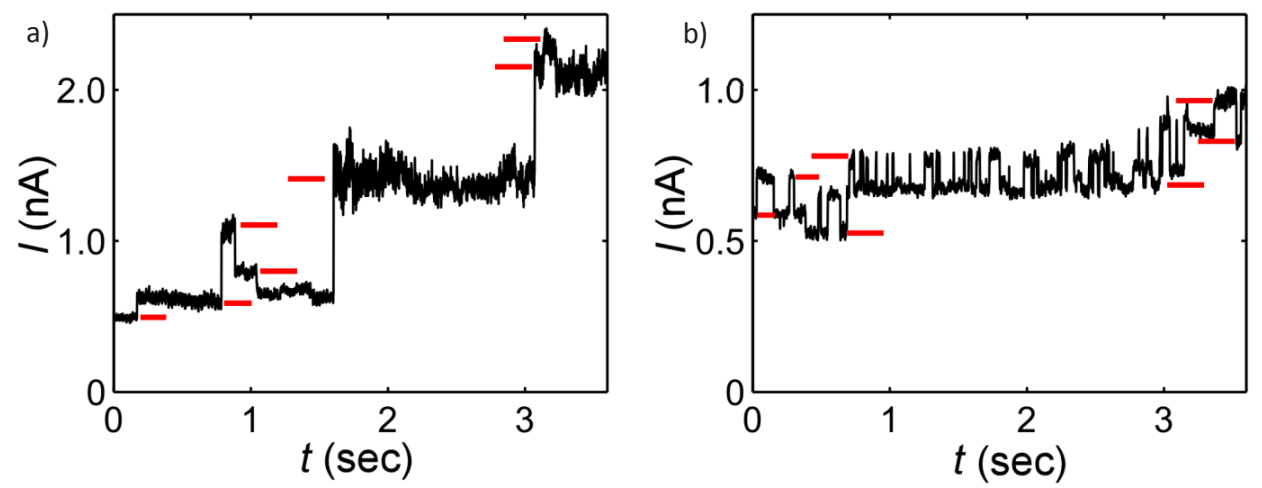

Figure 7.7: Current-time $(I-t)$ traces performed on a $\beta C D$ self-assembled monolayer at $77 \mathrm{~K}$ show at least seven different levels (a \& b). The sample biases were $-0.25 \mathrm{~V}$ (a) and $-1.25 \mathrm{~V}$ (b), respectively.

The recorded $I-t$ traces reveal that the switching process is often reversible. The involved intermediate states might however, vary from trace to trace. The reversibility of the process indicates that the molecule switches between different but rather stable conformational configurations. A similar behavior has been observed for azobenzene, ${ }^{[21]}$ which switches between two isomers.

\subsubsection{Dynamics of the $\beta C D$ Molecules}

Since the observed dynamics is not thermally induced the tunneling current and sample bias has been varied in order to confirm if the dynamics is induced by electrons that tunnel inelastically. The dynamics of the $\beta C D$ molecules were found to become increasingly richer, i.e. more levels, faster, and shorter residence times, upon increasing the tunneling 
current. The number of electrons that are required in order to induce a transition can in principle be determined by plotting the logarithm of the switching rate versus the logarithm of the current $\left(\operatorname{rate} \sim I^{N}\right)$. The slope gives the order, $N$, i.e. the number of electrons that is required in order to induce a transition. Since the $\beta C D$ molecules exhibit so many different levels, which are difficult to relate to specific conformational configurations of the molecule, it is not possible to determine the exact order of the transition. In Figure 7.8a the maximum number of observed levels (filled circles) and switching percentage (open circles) are plotted against the tunneling current at a sample bias of $10 \mathrm{mV}$ and a temperature of 77 $\mathrm{K}$. The switching percentage represents the percentage of traces where molecular switching has been observed. The increase in the switching percentage follows the higher switching rate at higher tunneling current. A clear trend, i.e. an increase in the number of levels with increasing current, is observed. It should be noted that even at such a low sample bias of only $10 \mathrm{mV}$, electron induced dynamics is observed. The latter implies that the excitation threshold should lie below $10 \mathrm{mV}$. In Figure $7.8 \mathrm{~b}$ the maximum number of levels is plotted against the tunneling current at a sample bias of $-0.5 \mathrm{~V}$. Also here an increase in the number of levels is observed with increasing tunneling current.
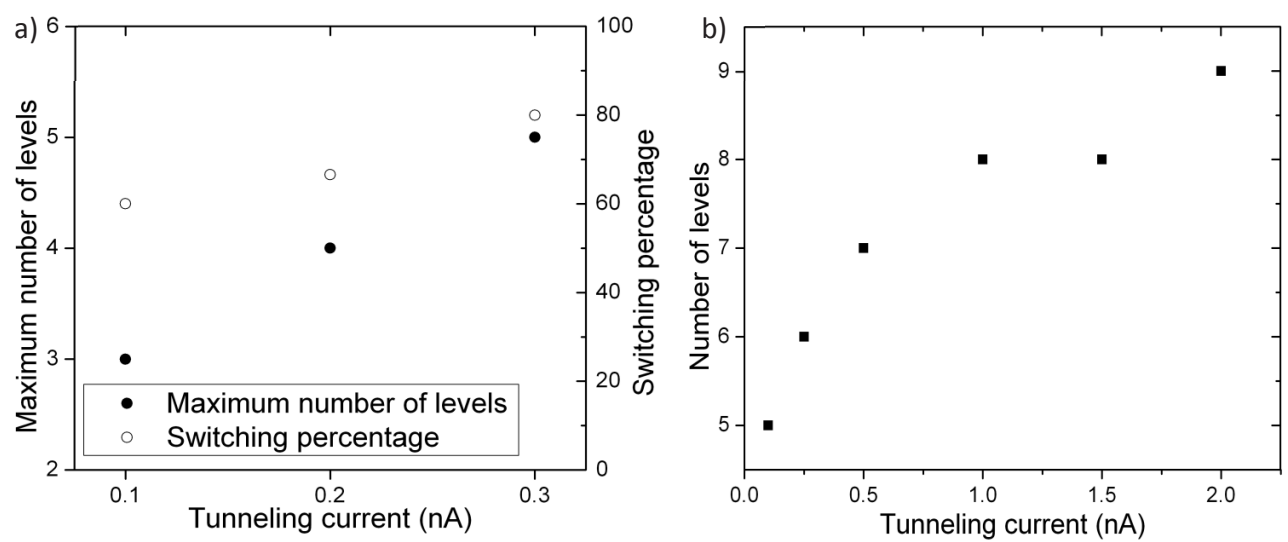

Figure 7.8: Maximum number of observed levels versus tunnelling current (filled circles) and switching percentage plotted against tunnelling current (open circles). The sample bias was set to $10 \mathrm{mV}$ and the data were recorded at $77 \mathrm{~K}$ (a). Maximum number of observed levels versus tunneling current. The sample bias was set to $-0.5 \mathrm{~V}$ and the data were recorded at $77 \mathrm{~K}(\mathrm{~b})$. 
Based on the experimental observations, the following scenario is proposed for the observed dynamics of the $\beta C D$ molecules. A small fraction of the electrons tunnel inelastically and is able to excite vibronic, i.e. stretching, wagging, bending or rotational, modes of the molecule, provided that the energy of the electron $(\mathrm{eV})$ is larger than the threshold for excitation $(\hbar \omega)$, i.e. $e V>\hbar \omega$. In a vibronic excited state the energy barrier to switch the molecule from configuration I to II (II to I) is reduced from its activation energy of $E_{\text {act }(I \rightarrow I I)}$ $\left(E_{\text {act }(I I \rightarrow I)}\right)$. Schematic energy diagram for such transitions is shown in Figure 7.9. With increasing current the probability to excite the $\beta C D$ molecule is enhanced and therefore it is likely that more excited levels will be observed in a given time interval. An increase of the sample bias also leads to enhanced dynamics for two reasons: (1) the efficiency of excitation increases with increasing electron energy and (2) for sufficient high energies (voltage) higher lying excited states can be reached.

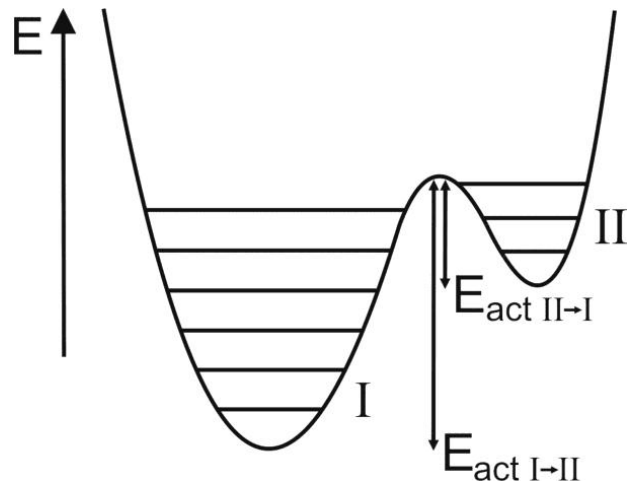

Figure 7.9: Schematic energy diagram of a molecule, which exhibits two conformational configurations (I and II). A transition from configuration I to II (II to I) will require an activation energy of $E_{\text {act }(I \rightarrow I)}\left(E_{\text {act(II } \rightarrow I}\right)$. The transition between them can be facilitated by the excitation of vibronic modes of the molecules. The separation between adjacent excited vibronic states is $\hbar \omega$.

Since the vibronic modes of the intramolecular bonds of organic molecules are usually larger than $\sim 10-30 \mathrm{meV}$, it is suggested that the excitation of the relatively heavy macrocycle of the $\beta C D$ molecule $(M=1135)$ induces the switching between the various conformational configurations of the molecule at low sample biases. Park et al. ${ }^{[22]}$ found similar vibrational modes arising in $\mathrm{C}_{60}$ molecules on $\mathrm{Au}$ surface, as $\mathrm{C}_{60}$ is bound to the surface by van der Waals interactions. Since the mass of a $\beta C D$ macrocycle is almost double the mass of a $\mathrm{C}_{60}$ molecule, it is expected that the vibrational quantum will be less than 5 
meV. However, the actual value will depend on the exact number of the sulfur moieties attached to the Au surface.

\subsection{Conclusion}

By using STM to study charge transport in a $\beta C D$ SAM, it has been possible to demonstrate that many different characteristics are seen at the single molecule level, that are averaged out and hence not apparent when performing $I(V)$ measurements on macroscopic junctions. Principally it was found that the $\beta C D$ molecules exhibit very rich dynamical behavior and conductance switching, thus indicating that different conformational states are present. The dynamics are found to be temperature independent, but increase with increasing tunneling current and sample bias, revealing that the conformational changes of the $\beta C D$ molecules are induced by electrons that tunnel inelastically. Even for sample biases as low as $10 \mathrm{mV}$ it was possible to induce a transition between the various conformational configurations of the $\beta \mathrm{CD}$ molecule. This study corroborates the complexity of charge transport that occurs in molecular tunneling junctions, and that still to this day, the field as a whole lacks a general understanding of charge transport at a molecular level. Therefore, great care must be taken when presenting and interpreting the conclusions based on $I(V)$ data obtained from macroscopic molecular junctions.

\subsection{Experimental Details}

\section{Materials:}

The synthesis of the heptathioether $\beta$-cyclodextrin $(\beta C D)$ molecules was carried out as previously described. ${ }^{[6,17]}$ All glassware used in the preparation of the $\beta C D$ monolayer was immersed in a piranha solution $\left(\mathrm{H}_{2} \mathrm{SO}_{4}\right.$ and $33 \% \mathrm{H}_{2} \mathrm{O}_{2}$ in a $3: 1$ ratio) for a minimum of 20 minutes. The glassware was then rinsed with large amounts of MilliQ water (18.2 M), and dried in an oven at $400 \mathrm{~K}$. Glass supported Au substrates $(\mathrm{Au}(250 \mathrm{~nm}) / \mathrm{Cr}(2.5 \mathrm{~nm}) / \mathrm{Glass})$ were purchased from arrandeeTM, Werther, Germany.

\section{Method:}

\section{SAM Formation:}

The Au substrates were rinsed with dichloromethane, dried and subsequently flame annealed in a hydrogen flame to obtain grains with large (111) oriented Au terraces. $\beta C D$ SAMs were 
prepared by immersing the $\mathrm{Au}$ substrates into a $0.1-1 \mathrm{mM}$ solution of $\beta \mathrm{CD}$ dissolved in a chloroform-ethanol (1:2) solution for 16 hours at $350 \mathrm{~K}$. The substrates were removed and rinsed thoroughly with three cycles of dichloromethane, ethanol and MilliQ water (18.2M) and then dried in a stream of nitrogen.

\section{Time-resolved Scanning Tunneling Microscopy measurements:}

The Scanning Tunneling Microscope (STM) and time-resolved measurements on the $\beta C D$ Self Assembled Monolayers (SAMs) were carried out with a RHK UHV700 and an Omicron Low Temperature STM (LT-STM) system under Ultra High Vacuum (UHV) conditions. The RHK UHV700 system was used for the measurements at room temperature, while the Omicron LT-STM was used for the measurements at $77 \mathrm{~K}$. For time-resolved measurements, the tunneling current was recorded as a function of time $(I(t)$ traces), while the feedback loop was switched off. Sampling frequencies in the range from 1-10 KHz were used. Electrochemically etched $W$-tips were used for all the measurements. A total of more than $900 I(t)$ traces were recorded at room temperature and at $77 \mathrm{~K}$. Each $I-t$ trace was acquired for $5-30$ seconds. About $75 \%$ of the $I(t)$ traces exhibited switching events between two or more current levels. Only a few percent of the $I(t)$ traces were useless due to the thermal drift of the tip with respect to the surface. These $I(t)$ traces were not included in our analysis.

\subsection{References}

[1] a)C. Joachim, J. K. Gimzewski, A. Aviram, Nature 2000, 408, 541-548; b)S. J. van der Molen, P. Liljeroth, J. Phys.-Condes. Matter 2010, 22, 133001; c)R. L. McCreery, A. J. Bergren, Adv. Mater. 2009, 21, 4303-4322; d)S. Karthauser, J. Phys.-Condes. Matter 2011, 23, 013001.

[2] a)C. A. Nijhuis, W. F. Reus, J. R. Barber, M. D. Dickey, G. M. Whitesides, Nano Lett. 2010, 10, 3611-3619; b)X. L. Li, J. Hihath, F. Chen, T. Masuda, L. Zang, N. J. Tao, J. Am. Chem. Soc. 2007, 129, 11535-11542; c)Y. Selzer, M. A. Cabassi, T. S. Mayer, D. L. Allara, J. Am. Chem Soc. 2004, 126, 4052-4053; d)H. B. Akkerman, P. W. M. Blom, D. M. de Leeuw, B. de Boer, Nature 2006, 441, 69-72.

[3] a)R. Haag, M. A. Rampi, R. E. Holmlin, G. M. Whitesides, J. Am. Chem. Soc. 1999, 121, 78957906; b)R. C. Chiechi, E. A. Weiss, M. D. Dickey, G. M. Whitesides, Angew. Chem. Int. Ed. 2008, 47, 142-144; c)T.-W. Kim, G. Wang, H. Lee, T. Lee, Nanotechnology 2007, 18, 315204; d)C. A. Richter, D. R. Stewart, D. A. A. Ohlberg, R. S. Williams, Appl. Phys. A-Mater. Sci. Process. 2005, 80, 1355-1362. 
Electron Induced Dynamics of Heptathioether $\beta$-cyclodextrin Molecules

[4] a)W. Haiss, H. van Zalinge, S. J. Higgins, D. Bethell, H. Hobenreich, D. J. Schiffrin, R. J. Nichols, J. Am. Chem. Soc. 2003, 125, 15294-15295; b)J. Reichert, R. Ochs, D. Beckmann, H. B. Weber, M. Mayor, H. von Lohneysen, Phys. Rev. Lett. 2002, 88, 176804; c)R. Temirov, A. Lassise, F. B. Anders, F. S. Tautz, Nanotechnology 2008, 19, 065401; d)L. Lafferentz, F. Ample, H. Yu, S. Hecht, C. Joachim, L. Grill, Science 2009, 323, 1193-1197; e)D. Kockmann, B. Poelsema, H. J. W. Zandvliet, Nano Lett. 2009, 9, 1147-1151; f)E. Leary, M. T. Gonzalez, C. van der Pol, M. R. Bryce, S. Filippone, N. Martin, G. Rubio-Bollinger, N. Agrait, Nano Lett. 2011, 11, 2236-2241; g)S.-Y. Jang, P. Reddy, A. Majumdar, R. A. Segalman, Nano Lett. 2006, 6, 2362-2367; h)A. M. Moore, B. A. Mantooth, Z. J. Donhauser, Y. X. Yao, J. M. Tour, P. S. Weiss, J. Am. Chem. Soc. 2007, 129, 10352-10353.

[5] K. S. Wimbush, W. F. Reus, W. G. van der Wiel, D. N. Reinhoudt, G. M. Whitesides, C. A. Nijhuis, A. H. Velders, Angew. Chem. Int. Ed. 2010, 49, 10176-10180.

[6] M. W. J. Beulen, J. Bügler, M. R. de Jong, B. Lammerink, J. Huskens, H. Schönherr, G. J. Vancso, B. A. Boukamp, H. Wieder, A. Offenhäuser, W. Knol, F. C. J. M. van Veggel, D. N. Reinhoudt, Chem. Eur. J. 2000, 6, 1176-1183.

[7] a)J. Huskens, M. A. Deij, D. N. Reinhoudt, Angew. Chem. Int. Ed. 2002, 41, 4467-4471; b)C. M. Bruinink, C. A. Nijhuis, M. Peter, B. Dordi, O. Crespo-Biel, T. Auletta, A. Mulder, H. Schonherr, G. J. Vancso, J. Huskens, D. N. Reinhoudt, Chem. Eur. J. 2005, 11, 3988-3996.

[8] a)C. A. Nijhuis, J. Huskens, D. N. Reinhoudt, J. Am. Chem. Soc. 2004, 126, 12266-12267; b)C. A. Nijhuis, F. Yu, W. Knoll, J. Huskens, D. N. Reinhoudt, Langmuir 2005, 21, 7866-7876; c)C. A. Nijhuis, B. A. Boukamp, B. J. Ravoo, J. Huskens, D. N. Reinhoudt, J. Phys. Chem. C 2007, 111, 9799-9810; d)C. A. Nijhuis, K. A. Dolatowska, B. J. Ravoo, J. Huskens, D. N. Reinhoudt, Chem. Eur. J. 2007, 13, 69-80.

[9] C. A. Nijhuis, J. K. Sinha, G. Wittstock, J. Huskens, B. J. Ravoo, D. N. Reinhoudt, Langmuir 2006, 22, 9770-9775.

[10] A. van Houselt, H. J. W. Zandvliet, Rev. Mod. Phys. 2010, 82, 1593-1605.

[11] A. D. Jewell, H. L. Tierney, A. E. Baber, E. V. Iski, M. M. Laha, E. C. H. Sykes, J. Phys.: Condens. Matter 2010, 22, 264006.

[12] Q. Liu, Y. Y. Zhang, N. Jiang, H. G. Zhang, L. Gao, S. X. Du, H. J. Gao, Phys. Rev. Lett. 2010, $104,166101$.

[13] a)A. van Houselt, R. van Gastel, B. Poelsema, H. J. W. Zandvliet, Phys. Rev. Lett. 2006, 97, 266104; b)T. F. Mocking, D. Stam, B. Poelsema, H. J. W. Zandvliet, Surf. Sci. 2010, 604, $2021-$ 2023; c)A. Saedi, A. van Houselt, R. van Gastel, B. Poelsema, H. J. W. Zandvliet, Nano Lett. 
2009, 9, 1733-1736; d)A. van Houselt, B. Poelsema, H. J. W. Zandvliet, J. Phys.-Condes. Matter 2010, 22, 264004.

[14] N. Battaglini, H. Klein, C. Hortholary, C. Coudret, F. Maurel, P. Dumas, Ultramicroscopy 2007, 107, 958-962.

[15] A. Kumar, R. Heimbuch, B. Poelsema, H. J. W. Zandvliet, J. Phys.-Condes. Matter 2012, 24, 082201.

[16] H. J. W. Zandvliet, A. van Houselt, in Annu. Rev. Anal. Chem., Vol. 2, 2009, pp. 37-55.

[17] M. W. J. Beulen, J. Bugler, B. Lammerink, F. A. J. Geurts, E. M. E. F. Biemond, K. G. C. van Leerdam, F. C. J. M. van Veggel, J. F. J. Engbersen, D. N. Reinhoudt, Langmuir 1998, 14, 6424-6429.

[18] J. V. Barth, H. Brune, G. Ertl, R. J. Behm, Physical Review B 1990, 42, 9307-9318.

[19] K. H. Gundlach, Solid-State Electron. 1966, 9, 949-957.

[20] L. Patrone, J. Soullier, P. Martin, Phys. Status Solidi B 2010, 247, 1867-1870.

[21] B. Y. Choi, S. J. Kahng, S. Kim, H. Kim, H. W. Kim, Y. J. Song, J. Ihm, Y. Kuk, Phys. Rev. Lett. 2006, 96, 156106.

[22] H. Park, J. Park, A. K. L. Lim, E. H. Anderson, A. P. Alivisatos, P. L. McEuen, Nature 2000, 407, 57-60. 


\section{Appendix}

In Chapters 4, 5 and 6 of this thesis generation one poly(propylene imine) (G1-PPI) and generation zero poly(amido amine) (G0-PAMAM) dendrimers of various terminal functionalities have been adsorbed on a $\beta C D$ SAM (i.e. supramolecular platform) in Eutectic Gallium Indium tunneling junctions to obtain a greater fundamental understanding of charge transport in molecular tunneling junctions. By performing statistically relevant numbers of current density vs. voltage $J(V)$ measurements $\pm 2.0 \mathrm{~V}$ and by applying voltage pulses at various biases over different periods of time it was possible to investigate many different parameters that can affect the charge transport characteristics. These parameters included the energies of the highest occupied molecular orbital (HOMO) levels of the dendrimer terminal functional moieties relative to the Fermi level of the electrodes, the position of the HOMO level within the tunneling junction, the density of the dendrimer layer, and the chemical interaction of the dendrimer with the EGaIn top electrode. Alongside these studies, three other methods were attempted to investigate the mechanism of charge transport in these supramolecular tunneling junctions. These studies were:

1) Using higher generations of PPI dendrimers, which changes the amount of terminal functional moieties, the number of moieties interacting with the supramolecular platform and the density and thickness of the dendrimer layer.

2) Increasing the bias window of the $J(V)$ measurements to $\pm 3.0 \mathrm{~V}$.

3) A top electrode other than EGaIn was used. Instead, the conductive polymer technique, where water-based suspension of poly(3,4-ethylenedioxythiophene) stabilized with poly(4-styrenesulphonic acid) (commonly known as PEDOT:PSS) was spin-coated on top of the supramolecular layer, which was followed by a $\mathrm{Au}$ layer being vapor deposited on top of the PEDOT:PSS layer.

In this appendix the results obtained for these three studies are presented in turn in sections A.1, A.2 and A.3 respectively. As some of these studies were unsuccessful or ongoing, the current status of these studies will also be stated. 


\section{A.1 EGaIn Supramolecular Tunneling Junctions Containing Higher Generations of Poly(propylene imine) Dendrimers}

Higher generations of dendrimers were investigated in EGaIn tunneling junctions to investigate two hypotheses:

1) The value of $R$ (where $R=|J(-2.0 \mathrm{~V})| /|J(+2.0 \mathrm{~V})|)$ will increase with the increase in the generation of dendrimer. This was hypothesized as the higher the generation of dendrimer the greater the number of terminal functional moieties. Therefore, as the generation increases there are more terminal functional moieties per dendrimer within the tunneling junctions, and all dendrimers are able to form three or more interactions with the $\beta C D$ SAM (with the number of interactions increasing with increase in generation for Fc terminated dendrimers) leading to a densely packed dendrimer layer on the supramolecular platform, with a coverage of approximately $100 \%$.

2) For junctions containing Fc terminated dendrimers, the absolute current density $(|J|)$ measured at $+2.0 \mathrm{~V}$ will decrease exponentially with the increase in generation of dendrimer, whilst $|J|$ measured at $-2.0 \mathrm{~V}$ will stay constant. This was hypothesized as the size of the dendrimer increases with increasing generation, and therefore so will the distance (d) between the bottom Au electrode and the top $\left(\mathrm{Ga}_{2} \mathrm{O}_{3}\right)$ EGaIn electrode. This, according to the molecular energy diagrams proposed in Chapter 4, Figure 4.5, the proposed mechanism of charge transport for these tunneling junctions at a positive bias is electron tunneling, which from the simplified version of the Simmons equation (Eq. 2.2) shows that $J$ decreases exponentially with increase in $d$, and the proposed mechanism of charge transport at a negative bias is electron hopping, which is independent of $d$.

In total eight EGaIn tunneling junctions were investigated. Seven supramolecular junctions contained Fc moieties: five junctions possessed single Fc terminal moieties, generation 2 (G2)-PPI-(Fc) 8 , generation 3 (G3)-PPI-(Fc) ${ }_{16}$, generation $4(\mathrm{G} 4)-P P I-(F c)_{32}$ and generation 5 (G5)-PPI-(Fc) 64 functionalized dendrimers immobilized on a $\beta C D$ monolayer to give the tunneling junctions $\quad \mathrm{Au}^{\mathrm{TS}}-\beta \mathrm{CDSAM} / \mathrm{G} 2-\mathrm{PPI}-(\mathrm{Fc})_{8} / /\left(\mathrm{Ga}_{2} \mathrm{O}_{3}\right) \mathrm{EGaIn} \quad(\mathrm{A} .1), \quad \mathrm{Au}^{\mathrm{TS}}$ $\beta C D S A M / G 3-P P I-(F c)_{16} / /\left(\mathrm{Ga}_{2} \mathrm{O}_{3}\right)$ EGaIn $\quad$ (A.2), $\quad \mathrm{Au}^{\mathrm{TS}}-\beta C D S A M / G 4-P P I-$ $(\mathrm{Fc})_{32} / /\left(\mathrm{Ga}_{2} \mathrm{O}_{3}\right)$ EGaIn (A.3) and $\mathrm{Au}^{\mathrm{TS}}-\beta \mathrm{CDSAM} / \mathrm{G} 5-\mathrm{PPI}-(\mathrm{Fc})_{64} / /\left(\mathrm{Ga}_{2} \mathrm{O}_{3}\right)$ EGaIn (A.4), respectively, and two junctions possessed BFc terminal moieties, G2-PPI-(BFc) 8 and 
G3-PPI-(BFc) ${ }_{16}$ functionalized dendrimers immobilized on a $\beta C D$ monolayer to give the tunneling junctions $\mathrm{Au}^{\mathrm{TS}}-\beta \mathrm{CDSAM} / \mathrm{G} 2-\mathrm{PPI}-(\mathrm{BFc})_{8} / /\left(\mathrm{Ga}_{2} \mathrm{O}_{3}\right)$ EGaIn (A.5) and $\mathrm{Au}^{\mathrm{TS}}$ $\beta C D S A M / G 3-P P I-(B F c)_{16} / /\left(\mathrm{Ga}_{2} \mathrm{O}_{3}\right)$ EGaIn (A.6), respectively (Figure A.1.1). The other junction did not contain Fc moieties, it consisted of a G3-PPI-(Ad) ${ }_{16}$ dendrimer immobilized on a $\beta C D$ monolayer to give the tunneling junction $\mathrm{Au}^{\mathrm{TS}}-\beta C D S A M / G 3-\mathrm{PPI}-$ $(\mathrm{Ad})_{16} / /\left(\mathrm{Ga}_{2} \mathrm{O}_{3}\right)$ EGaIn (A.7) (Figure A.1.1). All materials ${ }^{[1]}$ and tunneling junctions ${ }^{[2]}$ were prepared using the same method as shown in the experimental in Chapters 4, 5 and 6, and shown in Figures 4.1, 5.1) and 6.1).

For all the EGaIn tunneling junctions, statistically relevant numbers of data were accumulated by performing $J(V)$ measurements at biases of $\pm 2.0 \mathrm{~V}$ (Table A.1). The same statistical analysis presented in Chapters 4 and 5 was used to obtain the average $J(V)$ curve and the yield of working devices (Figure A.1.2) ${ }^{[3]}$. For the bias range of $\pm 2.0 \mathrm{~V}$, the yield of working junctions was found to be $\sim 90 \%$. 


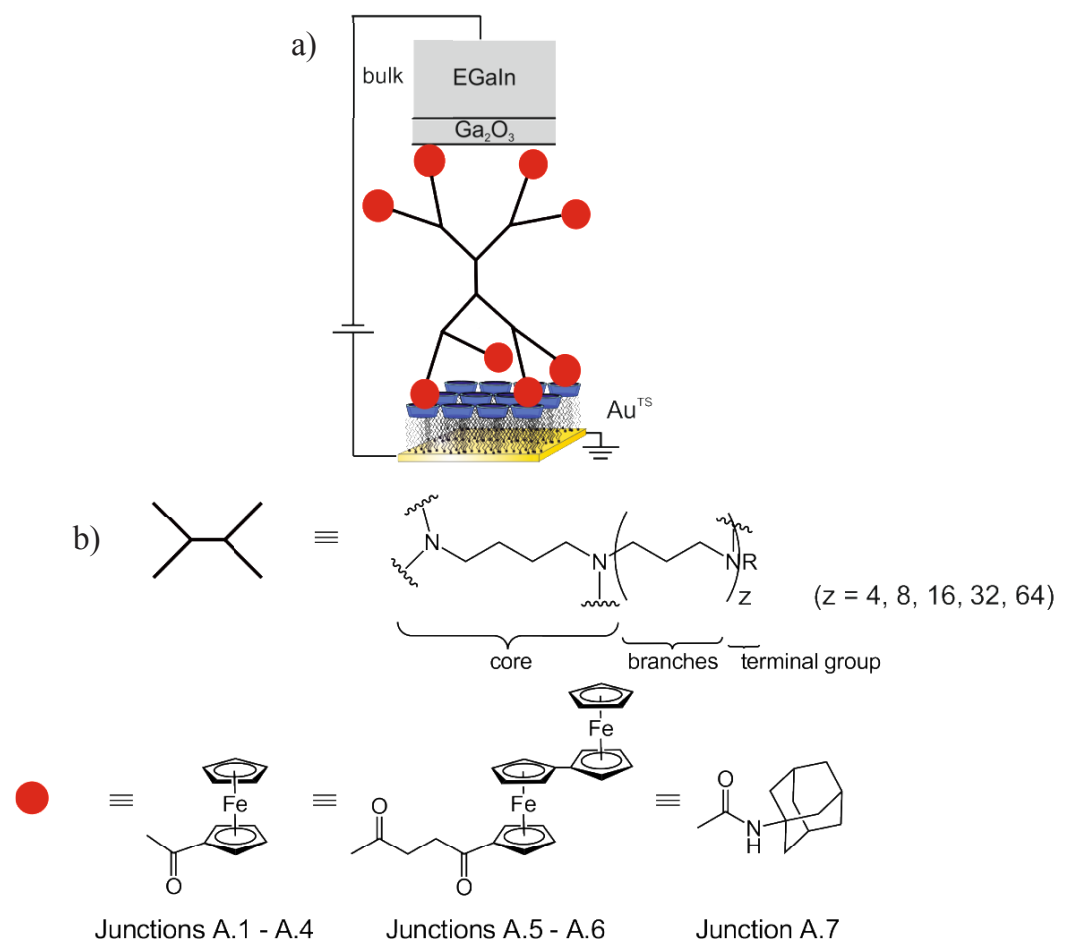

Figure A.1.1: (a) Schematic of the EGaIn supramolecular tunneling junction $\mathrm{Au}^{\mathrm{TS}}-\beta \mathrm{CDSAM} / \mathrm{G} 2-\mathrm{PPI}$ $(\mathrm{Fc})_{8} /\left(\mathrm{Ga}_{2} \mathrm{O}_{3}\right)$ EGaIn. (b) Molecular structure of the core of the poly(propylene) dendrimers, with the red sphere representing the functional groups at the periphery of the dendrimer, with the functionality (R) of the dendrimer corresponding to junctions A.1 - A.4, A.5 - A.6 and A.7, respectively. The $J(\mathrm{~V})$ measurements were performed by biasing the $\left(\mathrm{Ga}_{2} \mathrm{O}_{3}\right)$ EGaIn top-electrode and connecting the Au bottom-electrode to ground.

Hypothesis 1: The value of $R$ will increase with the increase in the generation of the dendrimer, was not verified as none of the junctions significantly rectified current (Table A.1, Figure A.1.2). The largest value of $R$ of $8.3\left(\sigma_{\log }=4.0\right)$ was found for junction A.5. It is unclear why the junctions containing larger generations of dendrimers give smaller values of $R$ than that found for junctions of smaller generations of dendrimers investigated in Chapters 4 and 5 .

Interestingly, junction A.7 gave the lowest value of $R\left(1.5\left(\sigma_{\log }=2.3\right)\right)$ (Table A.1). This is due to the adamantyl (Ad) dendrimer immobilized within the junction not possessing an accessible HOMO level, which is attributed to the Fc moieties. This trend matches the 204 
results found for all other supramolecular tunneling junctions containing dendrimers with Ad terminal moieties in Chapters 4 and 5.

Table A.1 Statistical overview of all $\left(\mathrm{Ga}_{2} \mathrm{O}_{3}\right)$ EGaIn junctions measured that contain higher generations of dendrimers.

\begin{tabular}{|c|c|c|c|c|c|c|c|c|c|}
\hline $\begin{array}{l}\text { Molecular } \\
\text { Junction }^{[a]}\end{array}$ & $\begin{array}{l}\text { No. of } \\
\text { Substrates }^{[b]}\end{array}$ & $\begin{array}{l}\text { No. of } \\
\text { Junctions } \\
\text { Created } d^{[c]}\end{array}$ & $\begin{array}{l}\text { No. of } \\
\text { working } \\
\text { Junctions } \\
(\%)^{[d]}\end{array}$ & $\begin{array}{l}\text { No. of } \\
\text { Shorts }\end{array}$ & $\begin{array}{l}\text { No. of } \\
\text { Unstable } \\
\text { Junctions } \text { s[] }^{[t]}\end{array}$ & $\begin{array}{l}\text { Total } \\
\text { Scans }{ }^{[g]}\end{array}$ & $\mathrm{R}^{[\mathrm{h}]}$ & Error $(68 \%)^{[i]}$ & $\begin{array}{l}\text { No. of } \\
\text { interactions } \\
\text { with } \beta C D \\
\text { SAM }^{\text {il }}\end{array}$ \\
\hline A.1 "G2-Fc" & 4 & 15 & $14(93)$ & 1 & 0 & 280 & 2.3 & $0.67-7.8$ & 3 \\
\hline A.2 "G3-Fc" & 7 & 34 & $30(88)$ & 3 & 1 & 600 & 3.9 & $1.2-12$ & 4 \\
\hline A.3 "G4-Fc" & 6 & 18 & $15(83)$ & 2 & 1 & 300 & 1.9 & $0.83-4.4$ & 7 \\
\hline A.4 "G5-Fc" & 5 & 13 & $12(92)$ & 0 & 1 & 240 & 4.6 & $1.9-11$ & 7 (or 8 ) \\
\hline A.5 "G2-BFe" & 3 & 16 & $16(100)$ & 0 & 0 & 320 & 8.3 & $2.1-33$ & 4 \\
\hline A.6 "G3-BFc" & 4 & 16 & $15(94)$ & 0 & 1 & 300 & 4.3 & $1.0-18$ & 4 \\
\hline A. 7 "G3-Ad" & 7 & 35 & $31(89)$ & 2 & 2 & 620 & 1.5 & $0.65-3.5$ & 4 \\
\hline
\end{tabular}

[a] A. $1=\mathrm{Au}^{\mathrm{TS}}-\beta \mathrm{CDSAM} / \mathrm{G} 2-\mathrm{PPI}-(\mathrm{Fc})_{8} / /\left(\mathrm{Ga}_{2} \mathrm{O}_{3}\right)$ EGaIn, A.2 $=\mathrm{Au}^{\mathrm{TS}}-\beta C D S A M / G 3-\mathrm{PPI}-(\mathrm{Fc})_{16} / /\left(\mathrm{Ga}_{2} \mathrm{O}_{3}\right) \mathrm{EGaIn}$, A.3 $=\mathrm{Au}^{\mathrm{TS}}-\beta \mathrm{CDSAM} / \mathrm{G} 4-\mathrm{PPI}-(\mathrm{Fc})_{32} / /\left(\mathrm{Ga}_{2} \mathrm{O}_{3}\right)$ EGaIn, A.4 = Au ${ }^{\mathrm{TS}}-\beta C D S A M / G 5-P P I-(\mathrm{Fc})_{64} / /\left(\mathrm{Ga}_{2} \mathrm{O}_{3}\right) \mathrm{EGaIn}$, A. $5=\mathrm{Au}^{\mathrm{TS}}-\beta \mathrm{CDSAM} / \mathrm{G} 2-\mathrm{PPI}-(\mathrm{BFc})_{8} / /\left(\mathrm{Ga}_{2} \mathrm{O}_{3}\right) \mathrm{EGaIn}, \mathrm{A} .6=\mathrm{Au}^{\mathrm{TS}}-\beta C D S A M / G 3-P P I-(\mathrm{BFc})_{16} / /\left(\mathrm{Ga}_{2} \mathrm{O}_{3}\right) \mathrm{EGaIn}$, $\mathrm{A} .7=\mathrm{Au}^{\mathrm{TS}}-\beta \mathrm{CDSAM} / \mathrm{G} 3-\mathrm{PPI}-(\mathrm{Ad})_{16} / /\left(\mathrm{Ga}_{2} \mathrm{O}_{3}\right) \mathrm{EGaIn} \quad[\mathrm{b}]=1 \mathrm{~cm} \times 1 \mathrm{~cm} \mathrm{Au}{ }^{\mathrm{TS}}$ surface on glass. [c] $=$ $\left(\mathrm{Ga}_{2} \mathrm{O}_{3}\right)$ EGaIn top electrode contacting the molecular monolayer immobilized on the $\mathrm{Au}^{\mathrm{TS}}$ surface. [d] $=$ junctions that gave 20 reproducible scans $\pm 2.0 \mathrm{~V}$ with the current measured being within $3 \sigma_{\log }$ of the $\mu_{\log }$ value, (number of working junctions/number of junctions created) [e] = a junction that produced an immediate ohmic response, or a junction that produced an ohmic response during the 20 scans $\pm 2.0 \mathrm{~V}$. [f] $=$ during the 20 scans $\pm 2.0 \mathrm{~V}$ the current measured varied greater than $3 \sigma_{\log }$ from the $\mu_{\log }$ value. $[\mathrm{g}]=$ a voltage sweep, $0.0 \mathrm{~V}$ $\rightarrow+2.0 \mathrm{~V} \rightarrow 0.0 \mathrm{~V} \rightarrow-2.0 \mathrm{~V} \rightarrow 0.0 \mathrm{~V}$. [h] $=|J(-2.0 \mathrm{~V})| /|J(+2.0 \mathrm{~V})| .[\mathrm{i}]=$ one log-standard deviation, $68 \%$ of the data is within one log standard deviation of the log-mean. [j] = amount of dendrimer terminal moieties that form host-guest interactions with the $\beta$ CDSAM, out of a possible total of four. $[\mathrm{k}]=$ surface coverage $(\%)$ of the dendrimer adsorbed to the supramolecular platform. ${ }^{[1 \mathrm{e}, 1 \mathrm{f}, 4]}$ 

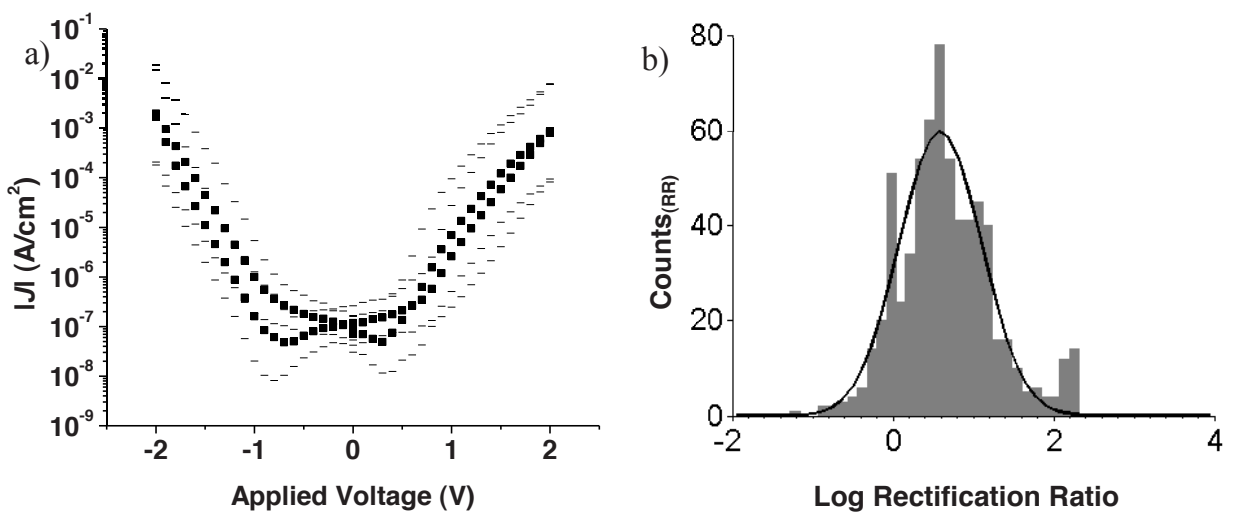

Figure A.1.2: A Semi-log plot of the averaged absolute current density vs. voltage ( $|J|$ vs. $V$ ) (a) and histogram of $\log$ rectification ratio $(R)$ (b) for junction A.6 = $\mathrm{Au}^{\mathrm{TS}}-\beta C D S A M / G 3-P P I-(B F c)_{16} /\left(\mathrm{Ga}_{2} \mathrm{O}_{3}\right)$ EGaIn. The error bars in the semi- $\log |J|$ vs. $V$ plots represent one $\log$ standard deviation from the log mean, and all histograms were fitted with a Gaussian curve to obtain the $\log$ mean $\left(\mu_{\log }\right)$ and $\log$ standard deviation $\left(\sigma_{\log }\right)$ thus allowing the calculation of $R(68 \%)$. 
Hypothesis 2: For junctions containing Fc terminated dendrimers, $|J|$ measured at $+2.0 \mathrm{~V}$ will decrease exponentially with the increase in generation of dendrimer, whilst $|J|$ measured at $-2.0 \mathrm{~V}$ will stay constant, was also not verified. Upon comparing $|J|$ measured at $+2.0 \mathrm{~V}$ and $|J|$ measured at $-2.0 \mathrm{~V}$, separately, for junctions A.1 - A.4 in this appendix and junction 4.1 in Chapter 4 (Figure A.1.3), it can be seen that neither trend is visible.
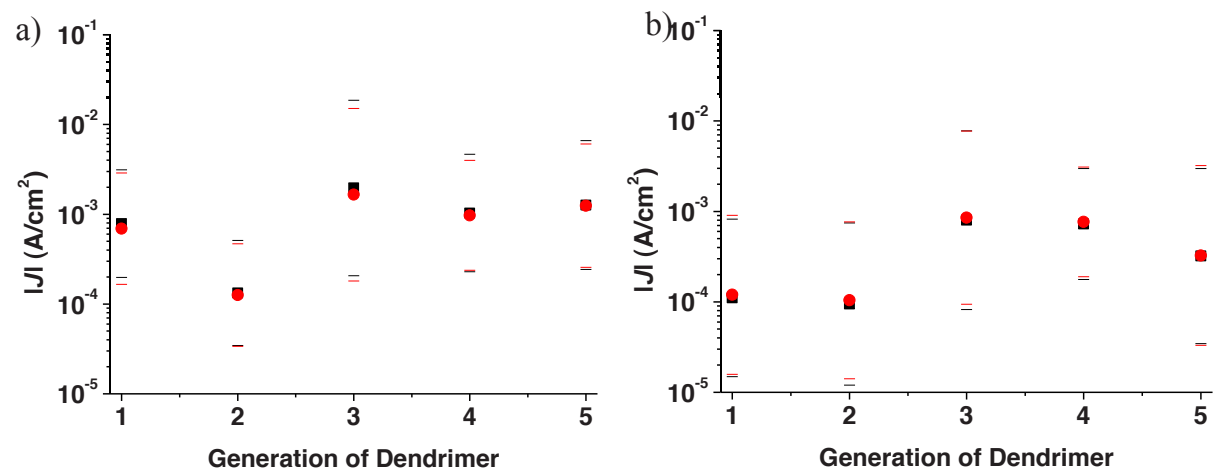

Figure A.1.3: Average value of $|J|$ measured at $-2.0 \mathrm{~V}$ (a) and $+2.0 \mathrm{~V}$ (b). Black squares represent $|J|$ measured during the forward scan $(0.0 \mathrm{~V} \rightarrow-2.0 \mathrm{~V}$ (a) and $(0.0 \mathrm{~V} \rightarrow+2.0 \mathrm{~V}(\mathrm{~b}))$, red squares represent $|J|$ measured during the reverse scan $(-2.0 \mathrm{~V} \rightarrow 0.0 \mathrm{~V}$ (a) and $+2.0 \mathrm{~V} \rightarrow 0.0 \mathrm{~V}$ (b)), with error bars representing one logstandard deviation, $68 \%$ of the data is within one log standard deviation of the log-mean.

In summary, neither of the two hypotheses (hypothesis 1: The value of $R$ will increase with the increase in the generation of dendrimer and hypothesis 2: For junctions containing Fc terminated dendrimers, $|J|$ measured at $+2.0 \mathrm{~V}$ will decrease exponentially with the increase in generation of dendrimer, whilst $|J|$ measured at $-2.0 \mathrm{~V}$ will stay constant) were verified. Further studies on the value of $R$ obtained in EGaIn tunneling junctions with higher generations of dendrimers, and the effects that ions have in these tunneling junctions will be carried out by the research group of Dr. Nijhuis at the National University of Singapore (NUS). 


\section{A.2 EGaIn Supramolecular Tunneling junctions at biases of $\pm 3.0 \mathrm{~V}$}

The density and thickness of the supramolecular layer made it possible to carry out $J(V)$ measurements at biases higher than that for any other molecular layer in EGaIn tunneling junctions. Other than the voltage pulses performed at $+2.5 \mathrm{~V}$ in Chapter $6, J(V)$ measurements were carried at biases of up to $\pm 3.0 \mathrm{~V}$. These measurements were carried out to investigate $J(V)$ characteristics that may only be seen at biases higher than $\pm 2.0 \mathrm{~V}$.

Junctions A.1 - A.5 and A.6 described in section A.1 of this appendix were investigated in this study. All materials ${ }^{[1]}$ and tunneling junctions ${ }^{[2]}$ were prepared using the same method as shown in the experimental in Chapters 4, 5 and 6, and shown in Figures 4.1, 5.1) and 6.1). Statistically relevant numbers of data were accumulated by performing $J(V)$ measurements at biases of $\pm 3.0 \mathrm{~V}$, with $J$ being measured every $0.15 \mathrm{~V}$. The same statistical analysis presented in Chapters 4 and 5 was used to obtain the average $J(V)$ curve. Although found to be reproducible and stable, the yield of working junctions for the bias range of $\pm 3.0 \mathrm{~V}$, was not calculated.

The value of $R$ for all junctions measured $\pm 3.0 \mathrm{~V}$ (where $R=|J(-3.0 \mathrm{~V})| /|J(+3.0 \mathrm{~V})|)$ was higher than $R$ found for all of the junctions measured at $\pm 2.0 \mathrm{~V}$ (where $R=\mid J(-2.0$ $\mathrm{V})|/| J(+2.0 \mathrm{~V}) \mid)$. The highest value of $R$ measured at $\pm 3.0 \mathrm{~V}$ was $82\left(\sigma_{\log }=5.6\right)$ for junction A.3 (Figure A.2.1a and b). Additionally, all junctions started displaying a reversible switching effect, i.e. acting as a memristor. $J$ measured during the forward scan $(0.0 \mathrm{~V} \rightarrow$ $3.0 \mathrm{~V}$ ) could be $\sim 1-2$ orders of magnitude higher than $J$ measured during the reverse scan $(3.0 \mathrm{~V} \rightarrow 0.0 \mathrm{~V})$ (Figure A.2.1a - c). The origin of the increase of $R$ at higher biases was investigated in Chapter 6, and was attributed to a chemical interaction occurring between the water and ions present in the supramolecular layer. This same reason is believed to be the origin of the 'memristor' effect.

In summary, when performing $J(V)$ measurements $\pm 3.0 \mathrm{~V}$ across the supramolecular tunneling junctions, the value of $R$ increases and the junctions show 'memristor like' behavior. The origin of this phenomenon is believed to be due to a chemical interaction occurring between the water and ions present in the supramolecular layer. Further studies of these phenomena are being carried out by the research group of Dr. Nijhius at NUS. 

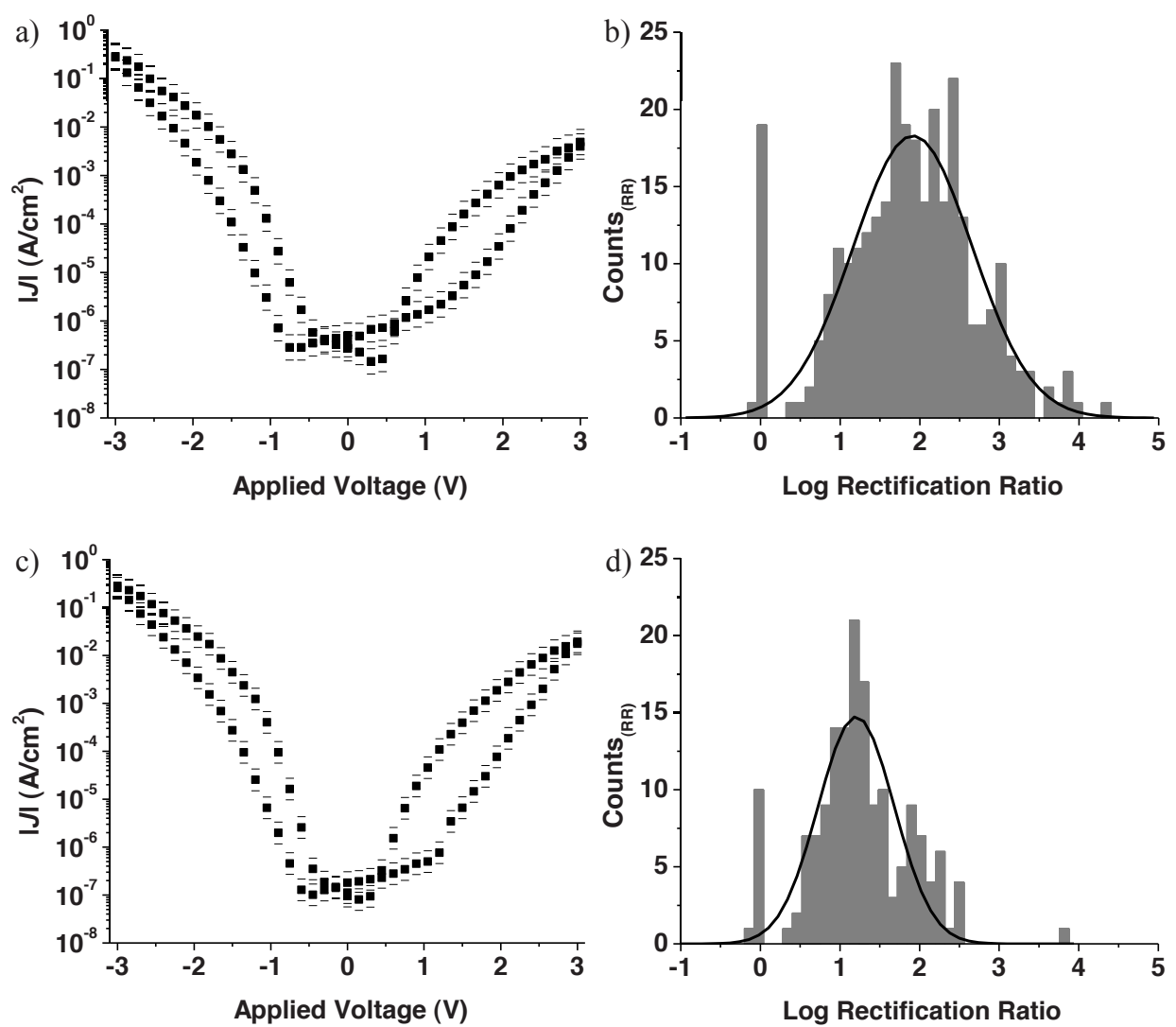

Figure A.2.1: A Semi-log plot of the averaged absolute current density vs. voltage ( $|J|$ vs. $V$ ) and histograms of $\log$ rectification ratio $(R)$ for junctions A.3 $=\mathrm{Au}^{\mathrm{TS}}-\beta \mathrm{CDSAM} / \mathrm{G} 4-\mathrm{PPI}-(\mathrm{Fc})_{32} / /\left(\mathrm{Ga}_{2} \mathrm{O}_{3}\right) \mathrm{EGaIn}(\mathrm{a}, \mathrm{b})$, and A.1 = $\mathrm{Au}^{\mathrm{TS}}-\beta \mathrm{CDSAM} / \mathrm{G} 2-\mathrm{PPI}-(\mathrm{Fc})_{16} / /\left(\mathrm{Ga}_{2} \mathrm{O}_{3}\right)$ EGaIn (c,d). The error bars in the semi-log $|J|$ vs. $V$ plots represent one $\log$ standard deviation from the $\log$ mean, and all histograms were fitted with a Gaussian curve to obtain the $\log$ mean $\left(\mu_{\log }\right)$ and $\log$ standard deviation $\left(\sigma_{\log }\right)$ thus allowing the calculation of $R(68 \%)$. 


\section{A.3 PEDOT:PSS Supramolecular Tunneling Junctions containing} Generation 1 and 3 Poly(propylene imine) Dendrimers.

As discussed in Chapter 2 and Chapter 3, the technique that is used to create the top electrode in two terminal molecular tunneling junctions can influence the $J(V)$ characteristics of the molecular layer. Therefore, it was of great interest to investigate the $J(V)$ characteristics of the supramolecular layer using a different tunneling junction technique. Thus, the supramolecular layer was investigated in PEDOT:PSS tunneling junctions.

Seven different tunneling junctions were investigated. Four supramolecular junctions contained Fc moieties: two junctions possessed single $\mathrm{Fc}$ terminal moieties, $\mathrm{Au}^{\mathrm{TS}}$ $\beta C D S A M / G 1-P P I-(F c)_{4} / / P E D O T: P S S-A u, \quad A u^{\text {TS }}-\beta C D S A M / G 3-P P I-(F c)_{16} / / \quad$ PEDOT:PSS$\mathrm{Au}$, and two junctions possessed $\mathrm{BFc}$ terminal moieties, $\mathrm{Au}^{\mathrm{TS}}-\beta C D S A M / G 1-P P I-$ $(\mathrm{BFc})_{4} / / \mathrm{PEDOT}: \mathrm{PSS}-\mathrm{Au}$ and $\mathrm{Au}^{\mathrm{TS}}-\beta \mathrm{CDSAM} / \mathrm{G} 3-\mathrm{PPI}-(\mathrm{BFc})_{16} / / \mathrm{PEDOT}: \mathrm{PSS}-\mathrm{Au}$. The other three junctions did not contain Fc moieties, thus acted as 'control junctions'. Two junctions contained Ad terminal moieties, $\mathrm{Au}^{\mathrm{TS}}-\beta \mathrm{CDSAM} / \mathrm{G} 1-\mathrm{PPI}-(\mathrm{Ad})_{4} / / \mathrm{PEDOT}: \mathrm{PSS} / \mathrm{Au}$ and $\mathrm{Au}^{\mathrm{TS}}$ $\beta C D S A M / G 3-P P I-(A d)_{16} /$ PEDOT:PSS/Au, whilst the other contained only the $\beta C D$ SAM, $\mathrm{Au}^{\mathrm{TS}}-\beta C D S A M / / \mathrm{PEDOT}: \mathrm{PSS} / \mathrm{Au}$.

The PEDOT:PSS tunneling junctions were fabricated in the laboratories of de Leeuw and co-workers, and thus were fabricated using the same method as previously published, ${ }^{[5]}$ and shown in Figure A.3.1. The $\beta C D$ SAM was immobilized on the Au bottom electrode using the same method presented in Chapter 7, and the dendrimer layers were absorbed to the $\beta C D$ SAM using the same method presented in Chapters 4, 5 and 6. Only investigative numbers of $J(V)$ measurements were performed $\pm 1.0 \mathrm{~V}$ and $\pm 2.0 \mathrm{~V}$. Junctions containing the bare $\beta C D$ SAM and G1-PPI dendrimers were found to give reproducible $J(V)$ measurements. However, due to fabrication problems, junctions containing G3-PPI dendrimers gave inconsistent results and thus will not be presented here. 


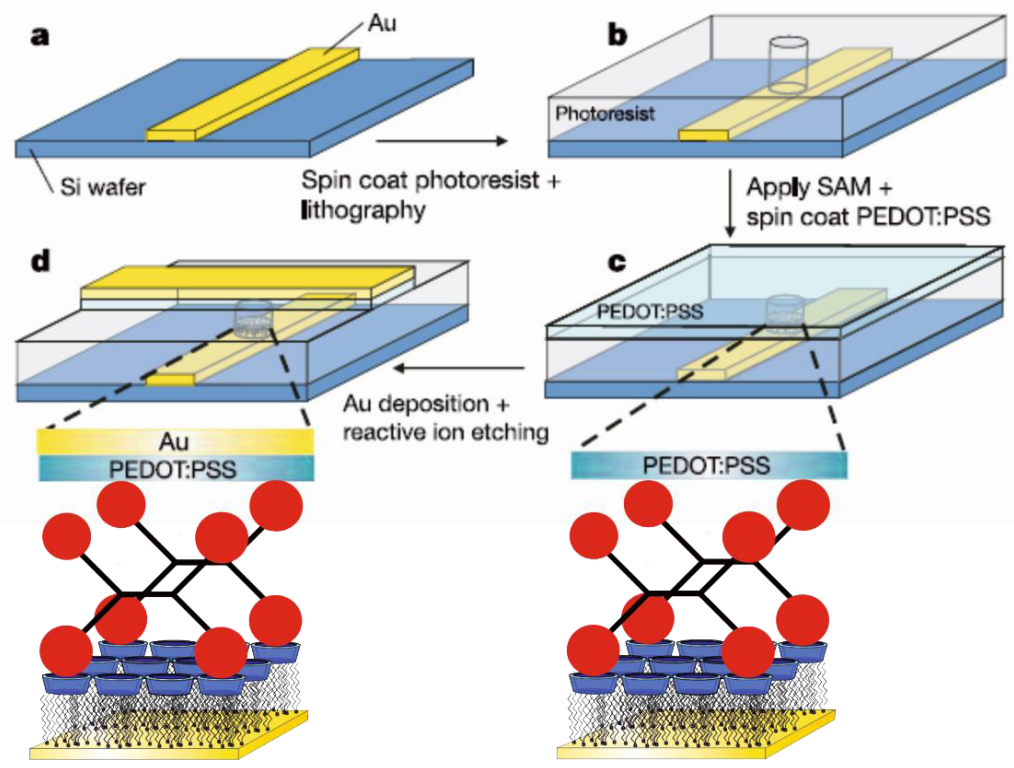

Figure A.3.1: a) Au electrodes are vapour deposited on a silicon wafer and a photoresist is spin-coated. b) Holes are photolithographically defined in the photoresist. c) The $\beta C D$ SAM and dendrimer layer are immobilized between the Au bottom electrode and the PEDOT:PSS top electrode. d) The junction is completed by vapour of Au through a shadow mask, which acts as a self-aligned etching mask during reactive ion etching of the PEDOT:PSS. The dimensions of these junctions range from 10 to $100 \mu \mathrm{m}^{2}$. (Figure adapted by permission from Macmillan Publishers Ltd: Nature, ${ }^{[5]}$ Copyright $~(c) 2006$.

From the $J(V)$ data obtained (Figures A.3.2 and A.3.3) it is very clear that the PEDOT:PSS supramolecular tunneling junctions do not rectify current in the same manner as the EGaIn supramolecular tunneling junctions. This phenomenon cannot be due to difficulties with dendrimer adsorption upon the $\beta C D$ SAM, as one characteristic trend seen is that all of the junctions containing dendrimers showed the same increase in $J$ measured compared to that of the bare $\beta C D$ SAM. Another trend seen is that the value of $J$ obtained in the PEDOT:PSS tunneling junctions is $\sim 4.5$ orders of magnitude higher than that found in the EGaIn tunneling junctions. Similar differences in $J$ have also been seen when comparing $J$ for alkanethiols in both of the tunneling junction techniques. ${ }^{[5-6]}$ As briefly mentioned in Chapter 2 and Chapter 3, the molecule-electrode interface is different for each tunneling junction technique, with the possibility of the PEDOT:PSS layer engulfing the SAM, and the presence of the semiconducting $\mathrm{Ga}_{2} \mathrm{O}_{3}$ layer on the skin of the EGaIn limiting the electrical 
conduction and causing chemical reactions to occur with the molecular layer. Whilst it is probable that both of these ill-defined components cause the variation of $J$ measured, it is also quite likely that these two different interfaces effect the ability of the molecules to rectify current. Interestingly, monolayers of ferrocene alkanethiolates, which have similar characteristics to the supramolecular tunneling junctions containing $\mathrm{Fc}$ terminated dendrimers, have been found to rectify current in EGaIn tunneling junctions and junctions where the top electrode was applied with a the Au foil layer, ${ }^{[3]}$ however they were not able to rectify currents in PEDOT:PSS tunneling junctions. ${ }^{[7]}$

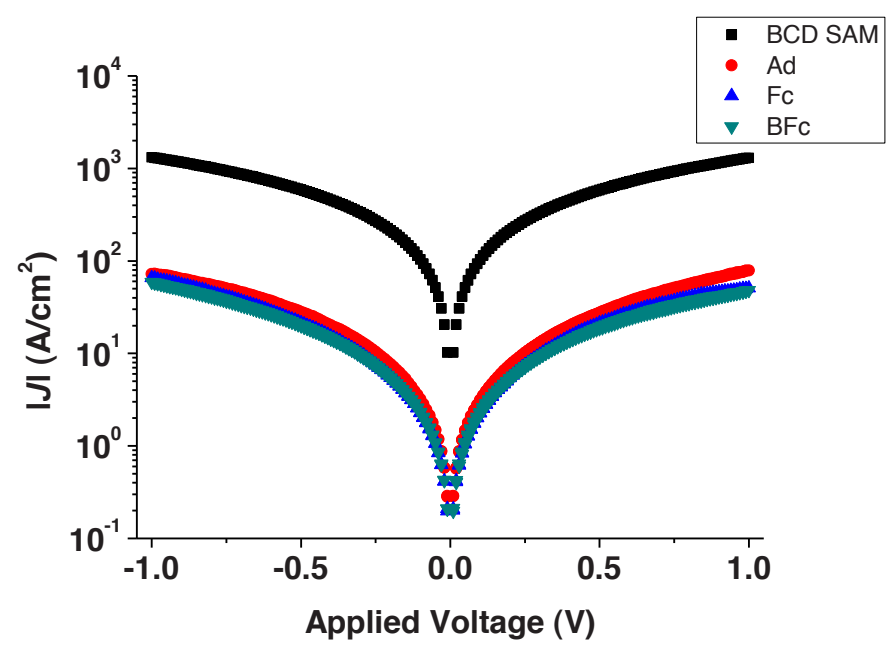

Figure A.3.2: Semi-log plot of $J(V)$ measurements $\pm 1.0 \mathrm{~V}$ performed across the junctions $\mathrm{Au}^{\mathrm{TS}}-\beta \mathrm{CDSAM}$ PEDOT:PSS/Au (black square), $\mathrm{Au}^{\mathrm{TS}}-\beta \mathrm{CDSAM} / \mathrm{G} 1-\mathrm{PPI}-(\mathrm{Ad})_{4} / \mathrm{PEDOT}: \mathrm{PSS} / \mathrm{Au} \quad$ (red circle), $\mathrm{Au}^{\mathrm{TS}}$ $\beta C D S A M / G 1-P P I-(F c)_{4} /$ PEDOT:PSS/Au (blue triangle) and $\mathrm{Au}^{\text {TS }}-\beta C D S A M / G 1-P P I-$ $(\mathrm{BFc})_{4} / \mathrm{PEDOT}: \mathrm{PSS} / \mathrm{Au}$ (green triangle) 


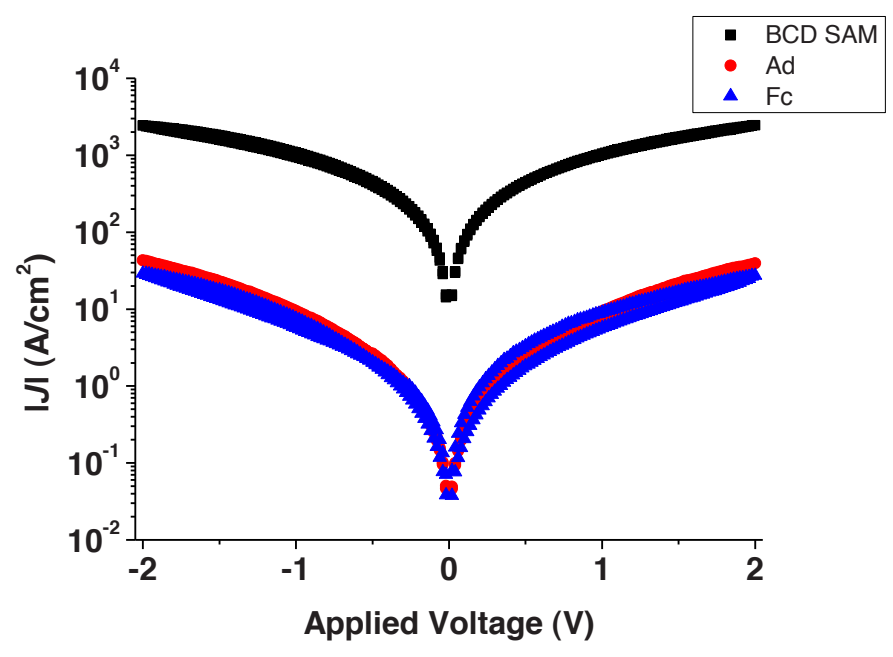

Figure A.3.3: Semi-log plot of $J(V)$ measurements $\pm 2.0 \mathrm{~V}$ performed across the junctions $\mathrm{Au}^{\mathrm{TS}}-\beta \mathrm{CDSAM}$ PEDOT:PSS/Au (black square), $\mathrm{Au}^{\mathrm{TS}}-\beta \mathrm{CDSAM} / \mathrm{G} 1-\mathrm{PPI}-(\mathrm{Ad})_{4} / \mathrm{PEDOT}: \mathrm{PSS} / \mathrm{Au}$ (red circle) and $\mathrm{Au}^{\mathrm{TS}}$ $\beta \mathrm{CDSAM} / \mathrm{G} 1-\mathrm{PPI}-(\mathrm{Fc})_{4} / / \mathrm{PEDOT}: \mathrm{PSS} / \mathrm{Au}$ (blue triangle)

In summary, the supramolecular layer when immobilized in PEDOT:PSS tunneling junctions, did not rectify current and gave higher values of $J$ than when immobilized in the EGaIn tunneling junctions. The difference in the value of $J$ measured and the ability of the molecules to rectify current is most likely due to differences between the molecule// $\mathrm{Ga}_{2} \mathrm{O}_{3}$ interface and the molecule//PEDOT:PSS interface. No further studies of the $J(V)$ characteristics of the supramolecular layer in PEDOT:PSS tunneling junctions are planned. 


\section{A.4 References}

[1] a)M. W. J. Beulen, J. Bugler, B. Lammerink, F. A. J. Geurts, E. M. E. F. Biemond, K. G. C. van Leerdam, F. C. J. M. van Veggel, J. F. J. Engbersen, D. N. Reinhoudt, Langmuir 1998, 14, 6424-6429; b)M. W. J. Beulen, J. Bügler, M. R. de Jong, B. Lammerink, J. Huskens, H. Schönherr, G. J. Vancso, B. A. Boukamp, H. Wieder, A. Offenhäuser, W. Knol, F. C. J. M. van Veggel, D. N. Reinhoudt, Chem. Eur. J. 2000, 6, 1176-1183; c)M. Baars, A. J. Karlsson, V. Sorokin, B. F. W. de Waal, E. W. Meijer, Angew. Chem. Int. Ed. 2000, 39, 4262-4265; d)I. Cuadrado, M. Moran, C. M. Casado, B. Alonso, F. Lobete, B. Garcia, M. Ibisate, J. Losada, Organometallics 1996, 15, 5278-5280; e)C. A. Nijhuis, J. Huskens, D. N. Reinhoudt, J. Am. Chem. Soc. 2004, 126, 12266-12267; f)C. A. Nijhuis, F. Yu, W. Knoll, J. Huskens, D. N. Reinhoudt, Langmuir 2005, 21, 7866-7876.

[2] R. C. Chiechi, E. A. Weiss, M. D. Dickey, G. M. Whitesides, Angew. Chem. Int. Ed. 2008, 47, 142-144.

[3] C. A. Nijhuis, W. F. Reus, G. M. Whitesides, J. Am. Chem. Soc. 2009, 131, 17814-17827.

[4] J. Huskens, M. A. Deij, D. N. Reinhoudt, Angew. Chem. Int. Ed. 2002, 41, 4467-4471.

[5] H. B. Akkerman, P. W. M. Blom, D. M. de Leeuw, B. de Boer, Nature 2006, 441, 69-72.

[6] M. M. Thuo, W. F. Reus, C. A. Nijhuis, J. R. Barber, C. Kim, M. D. Schulz, G. M. Whitesides, J. Am. Chem. Soc. 2011, 133, 2962-2975.

[7] Information obtained from a conversation with Auke Kronemeijer. 


\section{Summary}

This thesis describes research carried out in the field of Molecular Electronics. The aim was to form stable and reproducible molecular tunneling junctions and to obtain a fundamental understanding of the mechanism of charge transport across all the components and interfaces that comprise these tunneling junctions.

In this study a variety supramolecular tunneling junctions were created. The basis of these junctions was a self-assembled monolayer of heptathioether functionalized $\beta$-cyclodextrin $(\beta C D)$ formed on an ulta-flat Au surface, i.e., the bottom electrode. This gave a well-defined hexagonally packed $\beta C D$ monolayer, which was coined as a 'supramolecular platform'. Adsorbed on the supramolecular platform were dendritic molecules that varied in core structure and/or terminal functionality. To complete the junction, a top-electrode of eutectic gallium indium (EGaIn; see Chapter 3) was contacted to the dendrimers. By changing the chemical structure of the dendrimer in the junction, it was possible to study the mechanism of charge transport as a function of molecular structure. Statistical relevant numbers of current vs. voltage $I(V)$ measurements performed across the different supramolecular tunneling junctions allowed for the use of the rectification ratio $(R)$, where $R=|J(-2.0 \mathrm{~V})| /|J(+2.0 \mathrm{~V})|$, to compare the charge transport characteristics of the junctions with different dendritic molecules. This allowed for the formulation and verification of the mechanism of charge transport across the tunneling junctions (Chapters 4 and 5). Additionally, due to the excellent stability of the supramolecular tunneling junctions it was possible to apply voltage pulses for extended periods of time across the junctions. This allowed to investigate the influence that the top $\left(\mathrm{Ga}_{2} \mathrm{O}_{3}\right)$ EGaIn electrode has on the charge transport characteristics of the junction as a function of time and applied bias (Chapter 6). Finally, by using the tip of a Scanning Tunneling Microscope, instead of a top electrode of $\left(\mathrm{Ga}_{2} \mathrm{O}_{3}\right) \mathrm{EGaIn}$, it was possible to investigate the charge transport characteristics at the single molecule level, which are averaged out in large-area junctions (Chapter 7).

Chapter 1 gives a general introduction of how and why the field of molecular electronics exists and provides an overview of the work that is presented in the thesis. 
Chapter 2 gives a detailed literature overview in regards to the charge transport characteristics of the different types of self-assembled monolayers (SAMs) in two-terminal large-area $\left(>1 \mathrm{um}^{2}\right)$ molecular tunneling junctions fabricated using different strategies. Also introduced in Chapter 2 are each of the components and the interfaces that comprise a twoterminal molecular tunneling junction, with explanations on how they can affect the SAMs charge transport characteristics.

Chapter 3 begins with a discussion describing the different techniques that are used to create two-terminal large-area $\left(>1 \mathrm{um}^{2}\right)$ SAM based tunneling junctions and the advantages and disadvantages associated with each technique. This is followed by a detailed description of the EGaIn setup at the University of Twente, based on the EGaIn setup at Harvard University in the group of Prof. G. M. Whitesides. This includes a list of all parts, the positioning and wiring of the apparatuses, the calibration measurements and the possible improvements that could be made to future setups. The setup was determined to be very sensitive and stable, as it only had a current offset of $\sim 5 \mathrm{pA}$ and allowed for $J(V)$ measurements to be regularly performed for $\sim 2$ hours. A protocol is given to create an EGaIn tunneling junction and how to collect and statistically analyze statistically relevant numbers of $I(V)$ curves. The importance of collecting statistical large numbers of data to minimize the influence that defects within the tunneling junctions can have on the $I(V)$ data obtained is also discussed, along with a method to form ultra-flat Au surfaces which minimize the formation of defects in the SAM. Finally, the reproducibility of the EGaIn technique is shown, as the results obtained from the $I(V)$ (which can also be reported as $J(V)$ where $J=$ current density $\left.\left(\mathrm{A} / \mathrm{cm}^{2}\right)\right)$ measurements performed on the supramolecular tunneling junctions using the EGaIn setup in the laboratory of Whitesides and co-workers (Chapter 4) were reproduced with the EGaIn setup at the University of Twente.

In Chapter 4, the rectification ratio $(R)$ was used to compare the charge transport characteristics of three different terminally functionalized generation 1 (G1), poly(propylene) imine (PPI) dendrimers adsorbed on the supramolecular platform using the EGaIn technique. The three different terminal functionalities were adamantyl (Ad), ferrocene $(\mathrm{Fc})$ and bi-ferrocene $(\mathrm{BFc})$, giving the dendrimers G1-PPI-(Ad) $)_{4}, \mathrm{G} 1-\mathrm{PPI}-(\mathrm{Fc})_{4}$ and G1-PPI-(BFc) 4 . From statistical amounts of $J(V)$ data, the value of $R$ was determined to be $0.70\left(\sigma_{\log }=2.5\right), 7.7\left(\sigma_{\log }=3.1\right)$ and $1.7 \times 10^{2}\left(\sigma_{\log }=1.9\right)$ for the Ad, Fc and BFc terminal functionalized PPI dendrimers, respectively. Therefore, the value of $R$ obtained was dependent on the terminal functional moiety of the dendrimer and thus the observed 216 
rectification ratio was molecular in origin. As only the junctions that possessed $\mathrm{Fc}$ terminated PPI dendrimers were found to rectify current, it was hypothesized that rectification occurs in these tunneling junctions due to the $\mathrm{Fc}$ functional groups providing a low lying HOMO level with energies close to that of the Fermi levels of the electrodes, whilst being asymmetrically placed within the tunneling junction, close to, and coupled with the top $\left(\mathrm{Ga}_{2} \mathrm{O}_{3}\right)$ EGaIn electrode (Hypothesis 1). The difference in the value of $R$ obtained for the dendrimers G1-PPI-(Fc) $)_{4}$ and G1-PPI-(BFc) $)_{4}$ was hypothesized to be due to the G1$\mathrm{PPI}-(\mathrm{Fc})_{4}$ dendrimer forming a discontinuous monolayer on the supramolecular layer platform causing 'thin area defects', whereas G1-PPI-(BFc) formed a continuous monolayer, thus minimizing the formation of thin area defects (Hypothesis 2).

In Chapter 5, the two hypothesizes formulated in Chapter 4 were tested by changing the position of the Fc terminal functional moiety (hence, the position of the HOMO level) within the supramolecular tunneling junction spatially, and by changing the packing density of the dendrimer on the supramolecular platform intentionally. This was achieved by changing the core of the dendrimer from PPI to PAMAM, and by introducing a ethylene glycol linker between the dendritic core and the guest functionality. This controlled the effective distance between the terminal moieties which defines the numbers of interactions and binding strength of the dendrimers with the host surface. The two dendrimers used were a generation zero poly(amido amine) ferrocene functionalized dendrimer $\left(\mathrm{G} 0-\mathrm{PAMAM}-(\mathrm{Fc})_{4}\right)$ and a generation zero poly(amido amine) ethylene glycol linkerferrocene functionalized dendrimer (G0-PAMAM-((EG) $\left.\left.\left.)_{3}\right)-\mathrm{Fc}\right)_{4}\right)$. The (G0-PAMAM-((EG) $\left.\left.\left.)_{3}\right)-\mathrm{Fc}\right)_{4}\right)$ dendrimer was asymmetrically placed within the junction (as are all dendrimers due to the presence of the supramolecular platform), but did not have a terminal functional moiety placed close to and coupled with the top $\left(\mathrm{Ga}_{2} \mathrm{O}_{3}\right)$ EGaIn electrode, as they were all bound to the supramolecular platform and separated from the topelectrode by the dendritic core and ethylene glycol linker. As this junction was found not to significantly rectify current, giving a $R$ value of $3.1\left(\sigma_{\log }=2.3\right)$, and as the Fc terminated dendrimers investigated in Chapter 4 that did have a terminal functional moiety placed close to and coupled with the top $\left(\mathrm{Ga}_{2} \mathrm{O}_{3}\right)$ EGaIn electrode rectified current, hypothesis 1 was verified. The G0-PAMAM-(Fc) ${ }_{4}$ dendrimer which is known to form a continuous monolayer on the supramolecular platform did significantly rectify current, giving a $R$ value of 12 $\left(\sigma_{\log }=5.5\right)$. As this value of $R$ was slightly higher than that of the value of $R$ found for the 
G1-PPI-(Fc) $)_{4}$ dendrimer in Chapter 4, which forms a discontinuous monolayer on the supramolecular platform, these results showed corroboration with hypothesis 2 .

In Chapter 6 the excellent stability of the supramolecular tunneling junctions allowed for the experimental limitations of the EGaIn technique to be investigated. By applying a voltage pulse $>+1.0 \mathrm{~V}$ across the supramolecular tunneling junctions, it was possible to increase the value of $R$ obtained from the subsequent $J(V)$ measurements $\pm 2.0 \mathrm{~V}$ in all junctions, regardless of supramolecular and chemical structure. The amount that the value of $R$ increased was found to be dependent upon the voltage and the time of the voltage pulse, with the largest voltage pulse $(+2.5 \mathrm{~V})$, being applied for the longest period of time $(60 \mathrm{~min})$, giving the largest value of $R\left(6.4 \times 10^{4}\right)$. From characterizing all the components and interfaces of the supramolecular tunneling junction, it was suggested that the increase in the value of $R$ was due to the $\mathrm{Ga}_{2} \mathrm{O}_{3}$ layer on the surface of the EGaIn becoming thicker, likely due to a (electro-) chemical reaction with the supramolecular layer. The thick $\mathrm{Ga}_{2} \mathrm{O}_{3}$ layer will then form a Schottky barrier within the junction, causing the value of $R$ to increase. Therefore, these results show that depending on the experimental conditions the total value of $R$ found for tunneling junctions investigated with the EGaIn technique, may have a contribution from the increasing $\mathrm{Ga}_{2} \mathrm{O}_{3}$ layer. At lower voltages $(<+1.0 \mathrm{~V})$ this is negligible, but at higher voltages and prolonged scanning periods this can become a most significant contribution.

In Chapter 7 STM and STS were used to investigate the charge transport characteristics of the $\beta C D$ SAM (supramolecular platform) on $\mathrm{Au}$ at the single molecule level. These measurements revealed that under an applied bias, even as low as $10 \mathrm{mV}$, the $\beta C D$ molecules exhibit a very rich dynamical behavior, which was not apparent in the large-area junction (i.e. the EGaIn junction) studies. The dynamics were found to be temperature independent but increased with increasing tunneling current and sample bias. This suggested that the conformational changes of the $\beta C D$ molecules were induced by electrons that tunnel inelastically. These results clearly indicate the complexity of charge transport in molecular tunneling junctions at the single molecule level, which is generally overlooked or averaged out when determining the mechanism of charge transport for large-area junctions.

In the appendix, three different sets of experiments are discussed. In the first, $J(V)$ measurements were performed $\pm 2.0 \mathrm{~V}$ on the higher generations of PPI dendrimers, which included generation $1(\mathrm{G} 1)$ to generation 5 (G5) Fc-terminated dendrimers, generation 2 
(G2) and generation 3 (G3) BFc-terminated dendrimers and a G3 Ad-terminated dendrimer. None of the higher generations of the Fc- or BFc-terminated dendrimers gave higher values of $R$ than their corresponding G1 dendrimers investigated in Chapter 4. The largest value of $R$ obtained was $8.3\left(\sigma_{\log }=4.0\right)$ found for the junction containing the G2-PPI-(BFc $)_{8}$ dendrimer. The G3-PPI-(Ad) $)_{16}$ dendrimer gave a value of $R$ similar to that of its corresponding G1 dendrimer. Interestingly, increasing the thickness of the molecular layer within the tunneling junction by increasing the generation of the dendrimer, did not significantly influence the $|J|$ measured. In the second set of experiments the bias range of the $J(V)$ measurements was increased to $\pm 3.0 \mathrm{~V}$. The increase in the bias range caused the value of $R$ (where $\mathrm{R}=|J(-3.0 \mathrm{~V})| /|J(+3.0 \mathrm{~V})|$ to increase for all supramolecular junctions, with the highest value of $R$ measured being $82\left(\sigma_{\log }=5.6\right)$ for junctions containing the dendrimer G4-PPI-(Fc) $)_{32}$. Additionally, all junctions displayed a reversible switching effect, i.e acted as a memristor. However, due to the results obtained in Chapter 6, it is suspected that both of these observations can be attributed to the chemical interaction of the $\mathrm{Ga}_{2} \mathrm{O}_{3}$ layer with the supramolecular layer. In the third and final set of experiments, the supramolecular layer was immobilized in poly(3,4-ethylenedioxythiophene): poly(4-stryenesulphonic), or known as PEDOT:PSS, tunneling junctions. None of the three dendrimers immobilized on the supramolecular platform, of which were G1-PPI-(Ad) ${ }_{4}, \mathrm{G} 1$ $\mathrm{PPI}-(\mathrm{Fc})_{4}$ and G1-PPI-(BFc) $)_{4}$, were found to rectify current. However, all junctions containing dendrimers did show lower values of $|J|$ than the junctions which contained only the bare supramolecular platform, thus indicating that the dendrimers were indeed adsorbed to the supramolecular platform. Finally, the values of $|J|$ measured in all PEDOT:PSS tunneling junctions were $\sim 4.5$ orders of magnitude higher than that measured in the EGaIn tunneling junctions. These results clearly display how the charge transport characteristics can greatly depend on the technique used to form the tunneling junction.

In summary, by adsorbing dendrimers of different core types and terminal functionalities to a supramolecular platform in EGaIn tunneling junctions, it has been possible to create stable molecular tunneling junctions and to control the amount of rectification in the tunneling junction. The point of contact of the top $\left(\mathrm{Ga}_{2} \mathrm{O}_{3}\right) \mathrm{EGaIn}$ electrode with the molecular layer seems to be very important because the increase in thickness of $\mathrm{Ga}_{2} \mathrm{O}_{3}$ layer on the surface of the EGaIn, resulting in a Schottky barrier rather than a van der Waals interface, can possibly dominate the electrical characteristics of the junctions. Therefore, possible changes in the molecule-electrode interface should be taken into account when interpreting data 
obtained from EGaIn studies. Recent work carried out by Nijhuis et al, shows that when the top $\left(\mathrm{Ga}_{2} \mathrm{O}_{3}\right)$ EGaIn electrode is in contact with water and/or $\mathrm{H}_{3} \mathrm{O}^{+}$, the thickness of the $\mathrm{Ga}_{2} \mathrm{O}_{3}$ layer can easily increase, which provides a good explanation for the increase in rectification in the supramolecular tunneling junctions presented in Chapter 6. However, most other EGaIn tunneling junction studies to date have been performed on monolayers of hydrophobic molecules that are not stable at biases higher than $\pm 1.0 \mathrm{~V} / \pm 1.5 \mathrm{~V}$. Therefore, in these studies, possible changes in the chemical composition of the $\mathrm{Ga}_{2} \mathrm{O}_{3}$ layer are likely to be negligible. Additionally, by using STM it was possible to get a better understanding of the charge transport phenomena that occurs at the single molecule level, which is averaged out in the large-area junctions. Therefore, this study as a whole allowed for a greater fundamental understanding of the mechanism of charge transport across all components that comprise a molecular tunneling junction. 


\section{Samenvatting}

Dit proefschrift beschrijft onderzoek op het gebied van de moleculaire elektronica. Het doel van dit onderzoek was om stabiele en reproduceerbare moleculaire tunneljuncties te fabriceren en deze vervolgens te gebruiken om nieuwe inzichten te verwerven in het ladingstransport.

In dit onderzoek werden verscheidende supramoleculaire tunneljuncties gefabriceerd. De basis van deze juncties was een monolaag van heptathioether-gefunctionaliseerd $\beta$-cyclodextrine $(\beta C D)$ op een ultravlak goudoppervlak, dat tevens als een electrode fungeert.

De $\beta C D$ monolagen zijn goed gedefinieerd en hebben een hexagonale structuur (het zogeheten supramoleculaire platform). Op dit platform werden dendrimeren geadsorbeerd met verschillende dendritische kernen en eindgroepen. De uiteindelijke junctie werd gevormd door een elektrisch contact te maken met deze supramoleculaire structuur met een eutectische legering van gallium en indium (EgaIn; zie Hoofdstuk 3). Door de chemische structuur van de dendrimeren te variëren kon het ladingsoverdrachtmechanisme in deze juncties onderzocht worden als functie van de chemische samenstelling van de monolagen. Om tot betrouwbare stroom(I)-spanning(V) relaties (I(V)) te komen werden veel metingen geanalyseerd en vergeleken.

Deze metingen maakten het ook mogelijk om het ladingsoverdrachtmechanisme te onderzoeken als functie van de 'gelijkrichtingscoëfficient' $R(R=|J(-2.0 \mathrm{~V})| /|J(+2.0 \mathrm{~V})|$. Zie Hoofdstukken 4 en 5 voor details. De goede stabiliteit van de juncties maakte het zelfs mogelijk om de tijdsafhankelijke effecten van de spanning over deze juncties op de elektrische eigenschappen van deze juncties te onderzoeken (zie Hoofdstuk 6). Met rastertunnelmicroscopie (scanning tunneling microscopie, STM) was het mogelijk om de elektrische eigenschappen van een enkel molecuul te onderzoeken (zie Hoofdstuk 7).

Hoofdstuk 1 geeft een korte en algemene inleiding over moleculaire elektronica alsmede een kort overzicht van het onderzoek dat in dit proefschrift is beschreven. 
In hoofdstuk 2 wordt een uitgebreid literatuuroverzicht gegeven over ladingoverdracht in tunneljuncties bestaande uit zelf-geassembleerde monolagen (self-assembled monolayers, SAMs). De SAMs zijn gefabriceerd met verschillende technieken en de typische oppervlakte van de elektroden is groter dan $1 \mu^{2}$. In dit hoofdstuk worden ook de verschillende componenten geïntroduceerd alsmede het effect van deze componenten en contacten op de ladingsoverdracht.

Hoofdstuk 3 beschrijft de verschillende fabricagetechnieken om moleculaire juncties te maken met een oppervlak van $>1 \mu \mathrm{m}^{2}$ en de voor- en nadelen van elke methode. Dit hoofdstuk beschrijft ook in detail de zogeheten EGaIn opstelling van de Universiteit Twente. Deze opstelling werd opgezet naar voorbeeld van de EGaIn opstelling van Harvard University (Prof. G. M. Whitesides). De opstelling werd uitvoerig getest, gekalibreerd en een lijst van de gebruikte onderdelen, de opstelling zelf, en een protocol om deze opstelling te bouwen worden in detail beschreven. De opstelling was stabiel genoeg om juncties te onderzoeken gedurende tijdspannen van enkele uren met een nauwkeurigheid van ongeveer $5 \mathrm{pA}$. Het grote belang van een statistische analyse van de vele metingen, het gebruik van ultravlakke bodemelektodes, en de reproduceerbaarheid van de metingen worden ook uitvoerig behandeld.

Hoofdstuk 4 behandelt de gelijkrichtingsscoëfficient (rectification ratio, $R$ ) van drie verschillende supramoleculaire juncties met dendrimeren van de eerste generatie (G1) poly(propylene) imine (PPI) geadsorbeerd op een monolaag van de gastheermoleculen. De verschillende gasteindgroepen waren adamantyl (Ad), ferrocene $(\mathrm{Fc})$ en bi-ferrocene $(\mathrm{BFc})$, resulterend in G1-PPI-(Ad) $)_{4}, \mathrm{G} 1-\mathrm{PPI}-(\mathrm{Fc})_{4}$ en G1-PPI-(BFc) $)_{4}$ dendrimeren, en analyse van statistisch relevante hoeveelheden $J(V)$ metingen leverde gelijkrichtingscoëfficienten van, respectievelijk, $0.70\left(\sigma_{\log }=2.5\right), 7.7\left(\sigma_{\log }=3.1\right)$ en $1.7 \times 10^{2}\left(\sigma_{\log }=1.9\right)$. De waarde van $R$ hangt dus af van de moleculaire samenstelling van de juncties. Op basis van deze metingen werden twee hypotheses voorgesteld. De eerste hypothese is gebaseerd op de observatie dat $R$ alleen een grote waarde heeft wanneer er een hoogste bezet moleculair orbitaal (highest occupied molecular orbital, HOMO) met een energie dichtbij het Fermi-niveau van de electroden aanwezig. Het Fermi-niveau moet asymmetrisch gepositioneerd zijn ten opzichte van de elektroden en sterker gekoppeld zijn met een van de twee elektroden. De tweede hypothese verklaart het verschil in $R$ van de G1-PPI-(Fc) $)_{4}$ en G1-PPI-(BFc) $)_{4}$ juncties aan de hand van het verschil in de eigenschappen van de monolagen van de gastmoleculen op de 
gastheermoleculen: monolagen die niet goed gevuld zijn hebben defecten die $R$ reduceren, terwijl monolagen die goed gevuld zijn een hoge waarde van $\mathrm{R}$ hebben.

In Hoofdstuk 5 worden de twee hierboven beschreven hypotheses getest door de positie van de HOMO ten opzichte van de elektroden en de dichtheid van dendrimeermonolaag op de gasheermonolaag te variëren, en wel door de PPI dendrimeer te vervangen met PAMAM met en zonder een lange verbindsgroep van tetraehtylene glycol (EG) tussen de dendrimeer en de gastfunctionaliteit, oftewel G0-PAMAM- $(\mathrm{Fc})_{4}$ en PAMAM-((EG) $\left.)_{3} \mathrm{Fc}\right)_{4}$. Beide moleculen vormen een monolaag op de monolaag van de gastheermoleculen met een maximale dichtheid. De PAMAM- $\left.\left((\mathrm{EG})_{3}\right)-\mathrm{Fc}\right)_{4}$ heeft alle vier de Fc groepen verbonden met de gastheer moleculen en heeft dus geen Fc groepen die geen direct contact kunnen vormen met de EGaIn electrode maar zijn juist gescheiden van de EGaIn electrode door de dendrimeer kern en de lange verbindingsgroepen. De $R$ waarde voor juncties met deze moleculen was $3.1\left(\sigma_{\log }=2.3\right)$, hetgeen in overeenstemming is met de eerste hypothese. De monolagen op de gastheermonolaag met G0-PAMAM- $(\mathrm{Fc})_{4}$ dendrimeren gaven juncties met een $R$ van $12\left(\sigma_{\log }=5.5\right)$, hetgeen hoger is dan de symmetrische junctie maar dan met G1-PPI-(Fc) $)_{4}$ dendrimeren die een lagere bezettingsgraad hebben van de gastheermonolaag, hetgeen in overeenstemming is met de tweede hypothese.

Hoofdstuk 6 behandelt het tijdsafhankelijke gedrag van de junctions wanneer een spanning over deze juncties wordt aangelegd. De waarde van $R$ nam toe als functie van de aangelegde spanning en tijdsduur van de aangelegde spanning. De grootste toename vond plaats voor de hoogste aangelegde spanning $(+2.5 \mathrm{~V})$. Bij deze spanning nam $R$ na een uur toe tot $6.4 \times$ $10^{4}$. Deze toename was onafhankelijk van de moleculaire samenstelling van de juncties. Het is belangrijk op te merken dat de moleculaire structuur van deze monolagen niet veranderd gedurende de experimenten. De EGaIn electrode lijkt onder de gebruikte omstandigheden een steeds dikkere $\mathrm{Ga}_{2} \mathrm{O}_{3}$ laag te vormen. Deze laag vormt een Schottky contact in plaats van een Van der Waals contact, wat leidt tot een grote toename van $R$. Dus in studies van ladingstransport in moleculaire tunneling junctions met een hoge aangelegde spanning kan de galliumoxide laag een belangrijke rol spelen en de gemeten $R$ zelfs domineren, oftewel, een veel groter effect hebben dan de andere molecuaire componenten in de junction.

In hoofdstuk 7 worden de experimenten en resultaten beschreven van de monolagen gekarakteriseerd met rastertunnelingmicroscopie. Met deze techniek konden de elektrische 
eigenschappen van een enkel gastheermolecuul in de gastheermonolagen worden bepaald. Zelfs bij lage spanning $(10 \mathrm{mV})$ bleken de dynamische eigenschappen van het molecuul belangrijk te zijn. Deze dynamische eigenschappen bleken onafhankelijk van de temperatuur te zijn, maar sterk af te hangen van de aangelegde spanning hetgeen impliceert dat deze dynamica geïnduceerd wordt door elektronen die inelastisch tunnelen. Dit soort 'single molecule' effecten kunnen niet in juncties gemeten worden die bestaan uit ensembles van moleculen en het is dus belangrijk om de elektrische eigenschappen van de moleculaire juncties te onderzoeken met complementaire technieken.

De Appendix beschrijft drie verschillende experimenten. Ten eerste worden elektrische eigenschappen van juncties beschreven met hogere generaties PPI dendrimeren van G1 tot G5 met Ad, Fc, en BFc eingroepen en een aangelegd spanning van maximaal $\pm 2.0 \mathrm{~V}$. Geen van deze structuren leidde tot juncties met hoge $R$ waarden of significante veranderingen in de gemeten stromen. Ten tweede werden de elektrische eigenschappen gemeten van de juncties met hoge aangelegde spanningen van $\pm 3.0 \mathrm{~V}$ in plaats van $\pm 2.0 \mathrm{~V}$. Dit leidde tot een grote $R$ (met $R=|J|(-3.0 \mathrm{~V}) /|J|(+3.0 \mathrm{~V})$ van $82\left(\sigma_{\log }=5.6\right)$. Deze juncties hadden ook zogeheten memristieve eigenschappen; gebaseerd op de bevindingen van Hoofdstuk 6 zou dit met de vorming van een dikke laag gallium oxide kunnen samenhangen. Ten derde worden ook de elektrische eigenschappen beschreven van juncties met G1-PPI-(Ad), G1$\mathrm{PPI}-(\mathrm{Fc})_{4}$ en G1-PPI-(BFc) $)_{4}$ dendrimeren met een electrode van poly $(3,4-$ ethylenedioxythiophene): poly(4-stryenesulphonic), oftewel PEDOT:PSS, in plaats van EGaIn. Geen van deze juncties had een grote $\mathrm{R}$ waarde, hetgeen in tegenstelling is met de bevindingen beschreven in Hoofdstuk 4. Daarnaast waren de gemeten stromen van deze junction 4-5 orders groter dan de juncties met EGaIn elektroden. De precieze experimentele techniek om het ladingsoverdracht in moleculaire tunneljuncties te bestuderen is dus van groot belang.

Samenvattend, het onderzoek beschreven in dit proefschrift laat zien dat stabiele moleculaire tunneljuncties gemaakt kunnen worden met een supramoleculair platform en dat de elektrische eigenschappen, zoals $R$, van deze juncties bepaald kunnen worden door middel van variatie in de kern- en eindgroepen van de op het platform geïmmobiliseerde dendrimeren. Het contactpunt van de moleculen met de EGaIn electrode blijkt erg belangrijk omdat de groei van de $\mathrm{Ga}_{2} \mathrm{O}_{3}$ laag tot een Schottky contact met de moleculen kan leiden, in plaats van een Van der Waals contact, wat vervolgens de elektrische eigenschappen van de juncties domineert. Dus in de interpretatie en verklaring van de data van EGaIn studies 
moeten ook potentiële veranderingen in de molecuul-electrode contact in ogenschouw worden genomen. Wanneer de top-electrode reageert met water en $\mathrm{H}_{3} \mathrm{O}^{+}$kan zich makkelijk een laag gallium oxide vormen, zoals recentelijk bevestigd door Nijhuis et al., wat een goede verklaring geeft voor de dendrimeer junctions beschreven in dit proefschrift. In de meeste studies die tot op heden zijn beschreven door andere groepen werden voornamelijk monolagen bestudeerd die geen water of $\mathrm{H}_{3} \mathrm{O}^{+}$bevatten en die niet stabiel zijn wanneer er een gelijkspanning van hoger dan 1.0 tot $1.5 \mathrm{~V}$ wordt aangelegd; in deze studies zijn de potentiele veranderingen in de chemische samenstelling van het galliumoxide laag waarschijnlijk verwaarloosbaar. Met STM hebben we de elektrische eigenschappen van een enkel molecuul in een monolaag bepaald. Deze single molecule eigenschappen zijn niet te detecteren wanneer ensembles van moleculen worden doorgemeten. Dit onderzoek, zoals beschreven in dit proefschrift, resulteert in nieuwe en belangrijke inzichten op het gebeid van de moleculaire elektronica. 


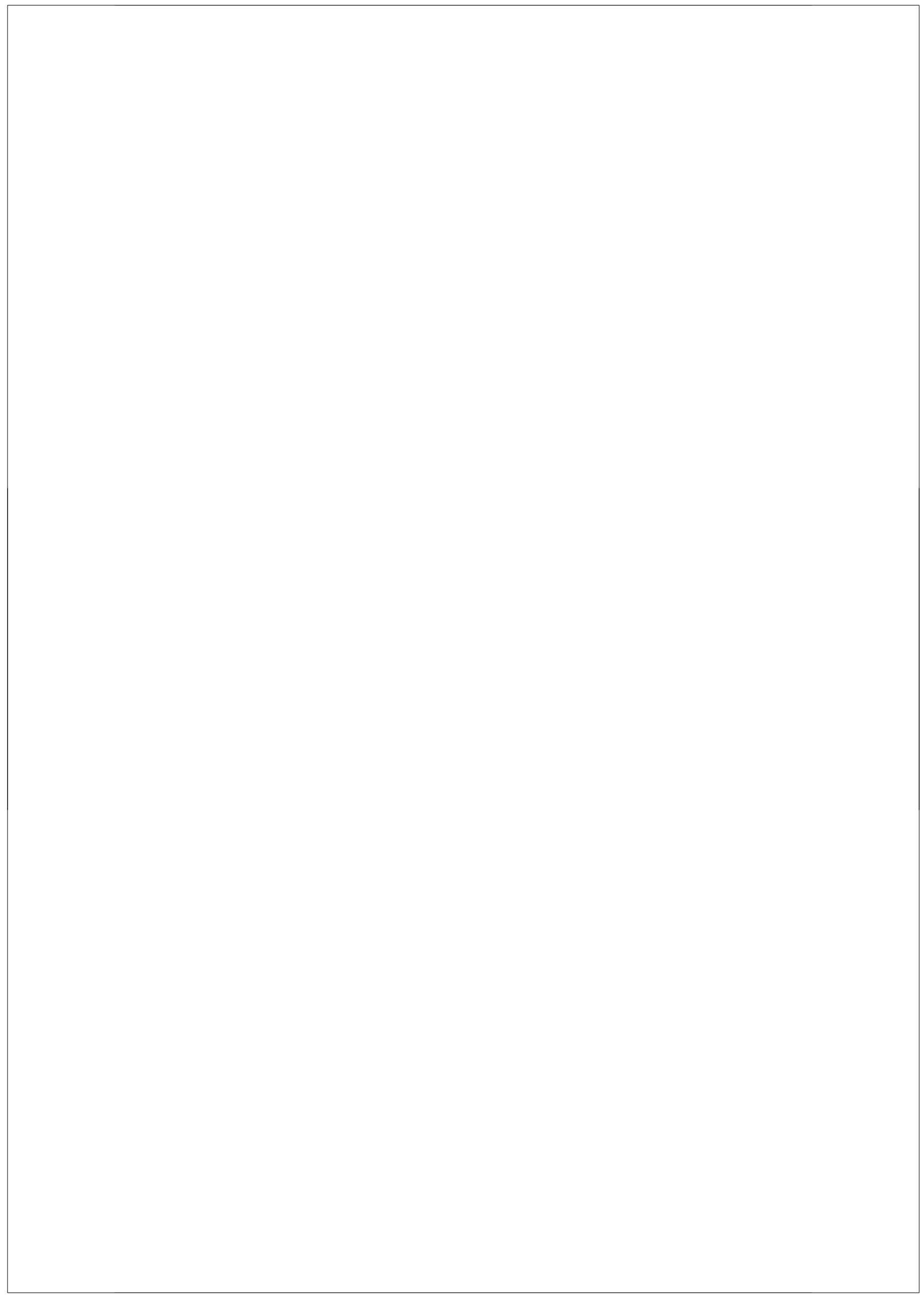




\section{Acknowledgements}

I would like to start by thanking my main supervisors. David Reinhoudt, from the beginning to the end you have been my promotor and I would like to thank you for always providing me with every opportunity available at your fingertips. Even after the first two international collaborations didn't yield nice results, you still showed faith in me and allowed me to go to Harvard, which was the launching point of my $\mathrm{PhD}$ thesis. Additionally, even though you retired half way through my second year, you have always been easily contactable and have played a crucial role in the completion of my thesis. Aldrik Velders, as of the beginning of my third year you became my direct supervisor, and now at the end you are also my promotor. It was quite interesting, a friend/colleague suddenly turned boss. I would like to thank you for your constant input throughout the project, the freedom you allowed me for the Harvard and Groningen collaboration and all of the after-hours corrections and experimental work, with the BFc synthesis coming directly to mind. I would also like to thank Christian Nijhuis for all of his involvement over the years. In Twente you introduced me to the world of dendrimers and $\beta C D$ monolayers, at Harvard you introduced me to the world of EGaIn and have always had a hands on involvement in my project. Therefore, I'm very happy that you have officially become my assistant promotor and I look forward to the future in Singapore.

I would also like to express my gratitude to my other supervisors. Wilfred van der Wiel for all of your general help and scientific discussions in the early years, and for your help with the EGaIn set-up in the later years. Bart Jan Ravoo, for his supervision during the first year and Riccardo Salvio for all of your synthetic help and discussions. Additionally, although never an official supervisor, I would also like to thank Jurriaan Huskens for always having an open door for discussions, and for inviting me to join his group discussions in the early years.

Throughout my PhD I have been lucky enough to participate in many scientific collaborations. I would like to thank Harold Zandvliet (whom I also classify as an unofficial supervisor) for all of the in depth scientific discussions and for his help with the samenvatting. In addition to Harold, I would also like to thank Avijit Kumar and Renne 
Heimbuch for the impressive STM measurements (Chapter 7). The breakthrough experiments of my $\mathrm{PhD}$ occurred during my time at Harvard (Chapter 4). Therefore, along with Christian, I would like to thank George Whitesides for the opportunity to work in his laboratories and for the scientific discussions and William Reus for all of his help and data analysis. I had a lot of fun, whilst being productive, when carrying out experiments with people from Groningen University (Appendix). For that I would like to thank Auke Kronemeijer, Hylke Akkerman, Ilias Katsouras, Hennie Valkenier, Dago de Leeuw and Bert de Boer. I was also involved in many collaborations that didn't make it into this thesis. Many thanks go to Benard Boukamp for all of the analysis and discussions on endless amounts of impedance data, William Dichtel, JC Olsen, Travis Gasa and Fraser Stoddart for the synthesis of the dendrimer rotaxanes and Maria Antonia Herrero Chamorro and Maurizio Prato for the adamantyl functionalized multi-walled nanotubes.

To my hard working Masters Students, Sven Krabbenborg and Natcha Chamnandechakun, and my hard working Erasmus student, Zbigniew Wrobel, although the work isn't in the thesis, I really appreciated the large amount of effort that you all put into challenging projects.

I would like to thank all of the technicians for all of their help throughout the years. Richard Egberink for all of your synthesis tips and tricks, computer support and Dutch admin. Marcel de Bruine for all of your help with ordering the parts for the EGaIn set-up and your masterful soldering skills. Martin Siekman (from Nanoelectronics) for his help with the Keithley and EGaIn set-up. Tieme Stevens for all the mass spec measurements and allowing me to invade the mass spec room. Bianca Snellink-Ruel for the help with the NMR and Regine van der Hee for the help with ordering.

Throughout the years there have been a few different secretaries in the group. I would like to thank Izabel Can-Katalanc, Nicole Haitjema, Geraldine Ramerman, Danielle Heskamp, Melissa Haartmans, and Marieke Van de Meer - Slotman for guiding me through the endless amount of admin.

I would also like to thank all of the other academics in the group over the years: Mercedes Crego Calama, Pascal Jonkheijm, Nathalie Katsonis, Jeroen Cornelissen, Wim Verboom and Melissa Koay for the wide range of discussions in the corridors and coffee room. 
From the SMCT group I would like to thank Raluca Fratila for all of the synthetic work that she did for my PhD project, with some of it presented in chapter 5. From this group I would also like to thank Albert Ruggi, Jealemy Galindo Milan, Peter Steunenberg, Arancha Gonzalez Campo, Shu-Han Hsu, Ignacio Perez Victoria, Victoria Gomez and Vijay Kumar Anuganti as we were the last few left. Thanks for all great scientific discussions and synthesis tips. Also thanks to Deniz Yilmaz from MNF for his synthetic help in the lab.

I would also like to thank those who helped me with the metal evaporation, from MNF Pieter Moonen and Sven Krabbenborg, from Nano-electronics Wouter Naber and from Mesoscale Chemical Systems Roald Tiggelaar.

Thanks also goes the everyone who I worked with in the AFM room in the early years of my project, Alberto Gomez Casado (images in Chapter 2), Eddie Benetti, In Yee Phang, Janet Acikgoz, Edit Kutnyanszky and Clemens Padberg. I would also like to thank Julius Vancso for his useful insight into Singapore.

I would also like to thank all of those for the good times in the labs, offices, during lunches, and outside of working hours, Henkino, Marloes, Alessio, Olga, Hans, Mirko, Fernando, Melanie, Carmen, Roald, Sachin, all the Polish, Spanish and Italian Erasmus students, Jordi, Carlo, Roberto, Emanuela, Angel, Anna, Francesca C, Raquel, Thomas, Rajesh, Mudassir, Deniz, Martin, Sobik, Francesca S, Lanti, Miguel and Marina, Vijay, Xing Yi, Chien-Ching, Srinidhi, Alessandro, Erhan, Martijn, Anne, Duan, Rick, Rik, Jenny, Shirish, Alex, Rindia, Liang, Laura, Nicolai, Supitch, Tushar, Alejandro, Tibor, Jasper, Timon, Janneke, Tom Bettina and Jiguang, Denis, Joost, Oya, Martine, Ana, Lourdes and of course Sarah.

I would also like to thank my paranimfs, Bart Lagerwaard and Raluca Fratila for helping me organize my defense. Finally, I would like to thank all my friends and family especially my Mum and Dad for the unconditional support they have given me during my $\mathrm{PhD}$. 


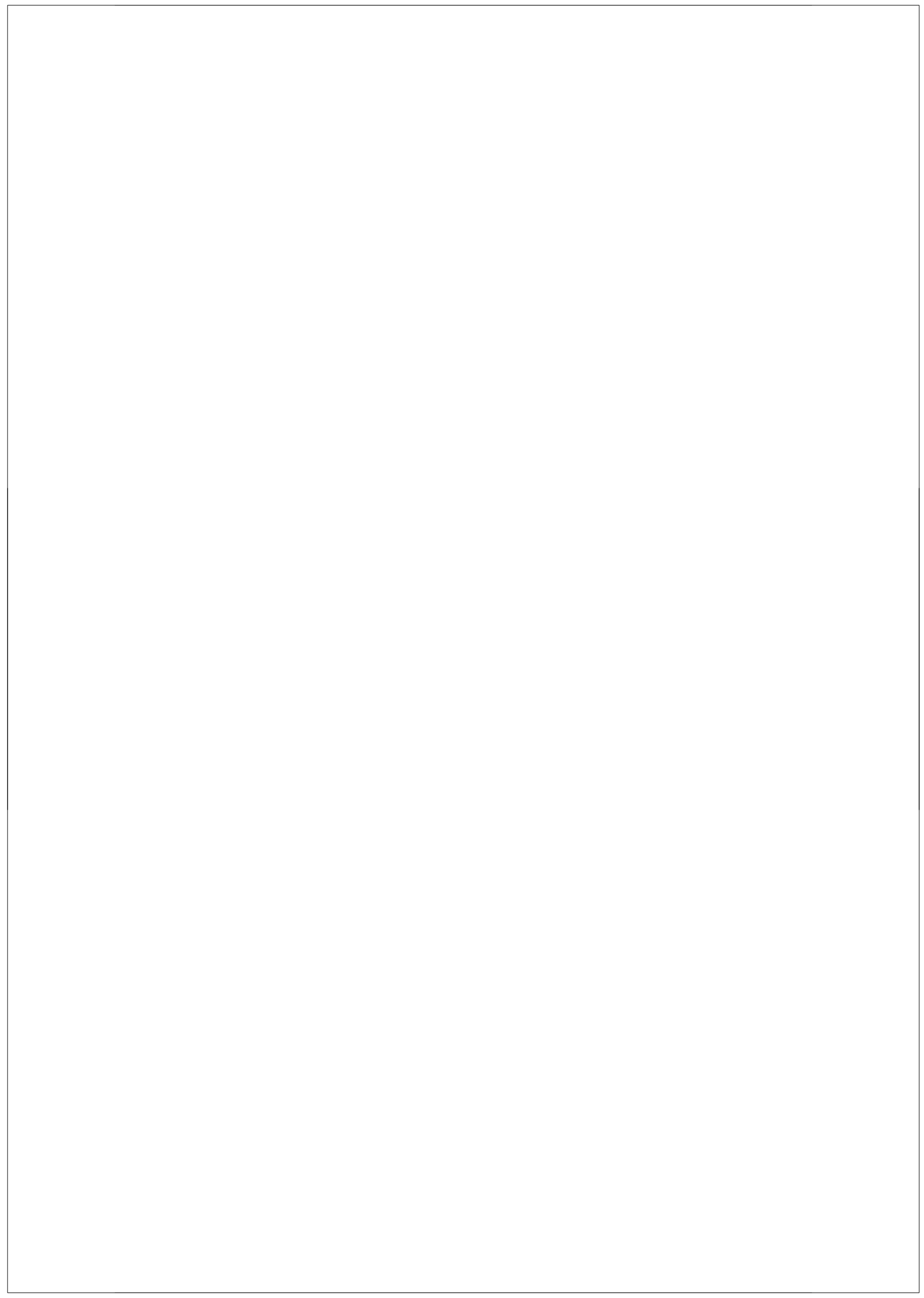




\section{About the Author}

Kim S. Wimbush was born in Campbelltown, South Australia, Australia, on June 3, 1984. He studied Nanotechnology (Honours) and majored in Biodevices at Flinders University, South Australia, Australia, where he received his degree in November 2005. During his honours project he worked on the development of a nanowire through DNA-directed attachment of Single Walled Carbon Nanotubes. The work was carried in the groups of Prof. J. Shapter and Prof. N. Voelcker at Flinders University, South Australia. In January 2006 he started as a PhD candidate in the Supramolecular Chemistry and Technology group of Prof. D. N. Reinhoudt at the University of Twente, The Netherlands. His PhD project was based in the field of molecular electronics, with a focus on supramolecular tunneling junctions. The work described in this thesis has been performed under the supervision of Prof. D. N. Reinhoudt and Prof. A. H. Velders, and co-supervision of Dr. C. A. Nijhuis. As of January 2013 he is a postdoctoral fellow in the group of Dr. C. A. Nijhuis at the National University of Singapore, Singapore. 


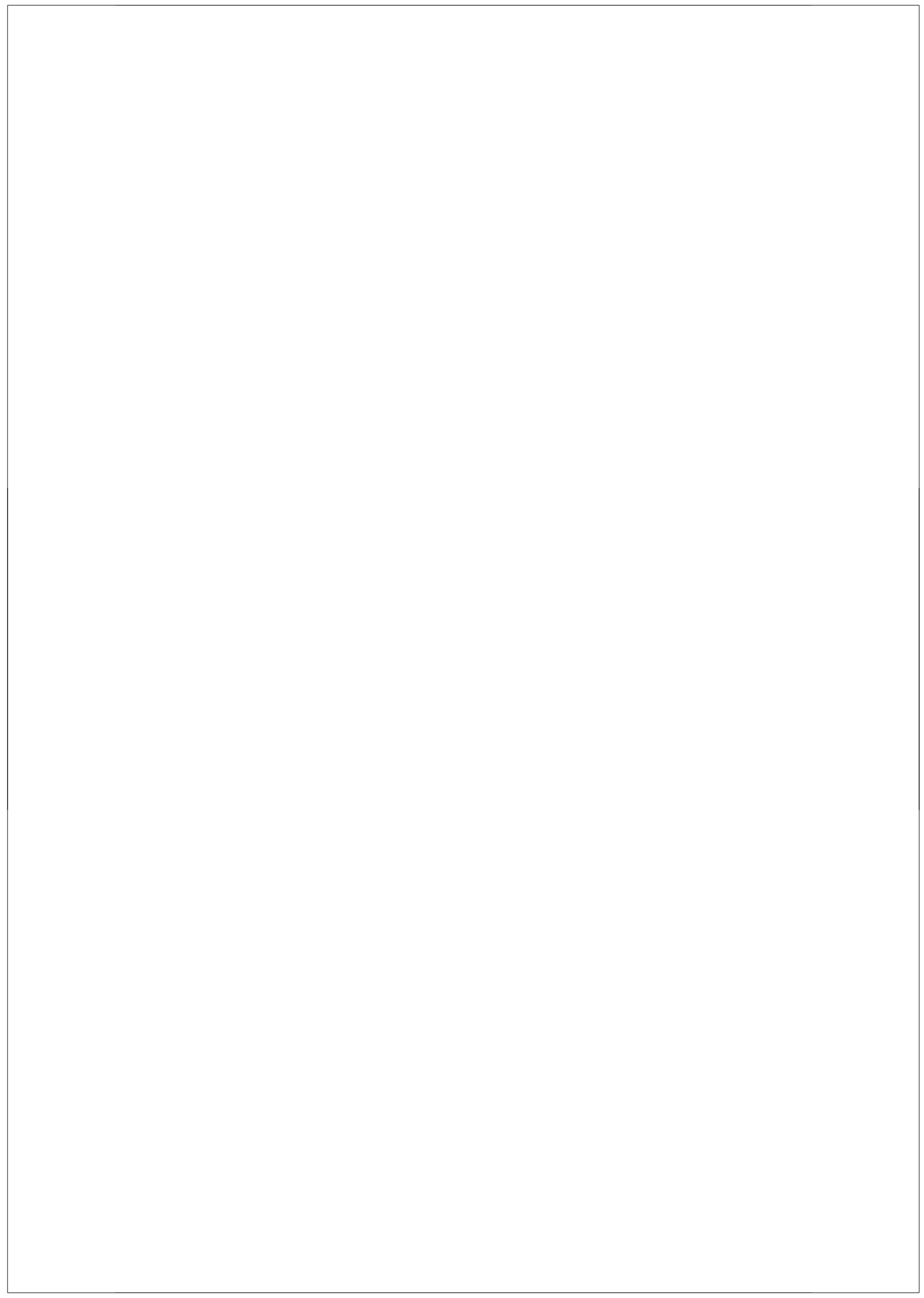

